Portland State University

PDXScholar

11-8-1995

\title{
Equivalent Relationship of Function-level Representation and Implementation of Unified Indexing of FFT Algorithms
}

Nee-Hua Cho

Portland State University

Follow this and additional works at: https://pdxscholar.library.pdx.edu/open_access_etds

Part of the Electrical and Computer Engineering Commons Let us know how access to this document benefits you.

Recommended Citation

Cho, Nee-Hua, "Equivalent Relationship of Function-level Representation and Implementation of Unified Indexing of FFT Algorithms" (1995). Dissertations and Theses. Paper 4876.

https://doi.org/10.15760/etd.6752

This Thesis is brought to you for free and open access. It has been accepted for inclusion in Dissertations and Theses by an authorized administrator of PDXScholar. Please contact us if we can make this document more accessible: pdxscholar@pdx.edu. 


\section{THESIS APPROVAL}

The abstract and thesis of Nee-Hua Cho for the Master of Science in Electrical and Computer Engineering were presented November 8, 1995, and accepted by the thesis committee and the department

COMMITTEE APPROVALS:

Marek Perkowski, Chair

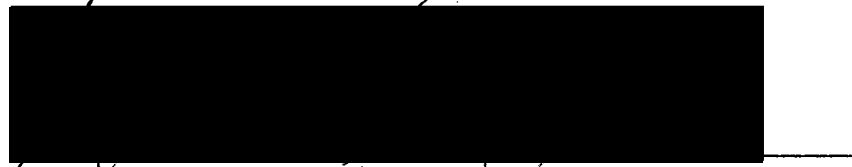

Michael A. Driscoll

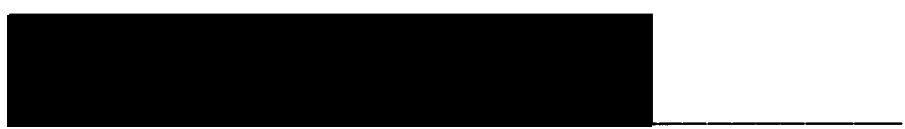

Thomas Schubert

Representative of the Office of Graduate Studies

DEPARTMENT APPROVAL:

Rolf Schaumann, Chair

Department of Electrical Engineering

ACCEPTED FOR PORTLAND STATE UNIVERSITY BY THE LIBRARY by
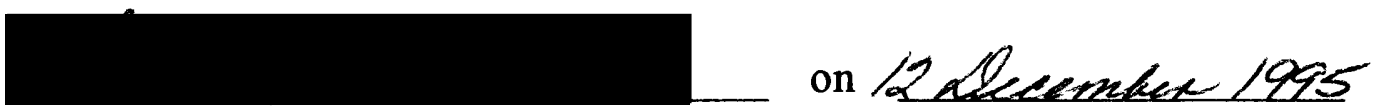
AN ABSTRACT OF THE THESIS OF Nee-Hua Cho for the Master of Science in Electrical and Computer Engineering presented November 8, 1995.

Title: Equivalent Relationship of Function-level Representation and Implementation of Unified Indexing of FFT Algorithms.

With the advance of the VLSI technology, the FFT algorithm has been pushed further in solving the multidimensional array signal processing in real time. Many DSP chip users have tried to find ways to improve addressing huge data in multidimension systems with minimum cost and maximum performance. However, there is no efficient method to address data for 1-D to M-D FFTs.

A methodology has been defined to conquer the addressing problem of M-D FFT. It is well known that the twiddle factor matrix of Discrete Fourier Transform (DFT) can be recursively factored into basic butterfly stage matrices. The matrix can be factored into three matrices practically specifying the input data, twiddle factor, and output data sequence of the Fast Fourier Transform (FFT). The equivalent relationship of these matrices will be introduced. The equivalent relationship for a variety of the FFT algorithms can be obtained by equivalent transformations. Furthermore, the multidimensional (M-D) FFT can be represented by the same vector-matrix form as the one-dimensional (1-D) FFT. In addition, the addressing sequences of the 1-D FFT is a subset of the M-D FFT. Therefore, the signal flow graph of the 1-D FFT can be used to describe that of the M-D FFT and all M-D indexing can be implemented by 1-D indexing. 
Finally, this unified indexing approach was implemented into the WinDSP software simulator. Examples of M-D FFTs implemented with the unified indexing method are simulated on the WinDSP and the computation performance were analyzed. From the benchmark analysis, the 2-D FFT applications implemented with unified methodology use less intstructions and the execution time is almost two times faster than the traditional method.

WinDSP is a software simulator that simulates the functional characteristics of Sharp LH9124/LH9320 DSP chip set. It intended to manage the complete development of DSP applications, from conceptualization and experimentation, to verification of unified indexing for 1-D to M-D FFT. It is also intended for system development, where hardware can be implemented for the design. 
EQUIVALENT RELATIONSHIP OF FUNCTION-LEVEL

REPRESENTATION AND IMPLEMENTATION OF

UNIFIED INDEXING OF FFT ALGORITHMS

\author{
by \\ NEE-HUA CHO
}

A thesis submitted in partial fulfillment of the requirements for the degree of

\author{
MASTER OF SCIENCE \\ in \\ ELECTRICAL AND COMPUTER ENGINEERING
}

Portland State University

1995 


\section{ACKNOWLEDGEMENTS}

First and foremost, I wish to dedicate this thesis to my wife, Rhonda Cho. She put up with all of my quirks and ranting while I wrote this thesis. No word can express my deep appreciation to my wife for all the work she did, especially, taking care of our son, being a homemaker and going to school at the same time. She helped console me through periods of utter chaos and discouragement. Her love, support, patience and understanding has made the writing of this thesis possible. Thanks, Sweetheart!

I would also like to express my gratitude to my adviser, Dr. Marek A. Perkowski, who has patiently guided me through this endeavor, allowing me to be ambitious and creative, and whose unwavering support guided me through the most trying times. His encouragement has bolstered my spirit and inspired me to continue working on this subject even after the completion of this thesis.

Additionally, I wish to thank Dr. Michael Driscoll and Dr. Thomas Schubert for their valuable comments, suggestions and critiques which have improved and strengthen my thesis work.

Finally, I am deeply appreciative and my most heartfelt thanks go to Dr. C. J. Ju who has patiently offered his invaluable time, support, encouragement, advice, and suggestions in the course of preparing and writing this thesis.

Vancouver, Washington 
TABLE OF CONTENTS

PAGE

ACKNOWLEDGEMENTS $\ldots \ldots \ldots \ldots \ldots \ldots \ldots \ldots \ldots \ldots \ldots \ldots$ iii

LIST OF TABLES $\ldots \ldots \ldots \ldots \ldots \ldots \ldots \ldots \ldots \ldots \ldots \ldots \ldots \ldots \ldots \ldots \ldots \ldots \ldots$

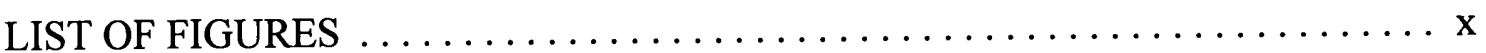

\section{CHAPTER}

I INTRODUCTION $\ldots \ldots \ldots \ldots \ldots \ldots \ldots \ldots \ldots \ldots \ldots \ldots \ldots$

Algorithm Analysis, Derivation and Mapping $\ldots \ldots \ldots \ldots \ldots 3$

1-D FFT Derivation and Analysis ............. 4

Unified Indexing for M-D FFTs $\ldots \ldots \ldots \ldots \ldots \ldots \ldots$

Problem Statement ...................... 5

Goals and Design Development Methodology........... 7

Organization of this Thesis................... 8

II FUNDAMENTALS OF DIGITAL SIGNALS ............ 11

Overview of Fast Fourier Transform $\ldots \ldots \ldots \ldots \ldots \ldots \ldots$

Formation of the FFT $\ldots \ldots \ldots \ldots \ldots \ldots \ldots \ldots \ldots \ldots \ldots$

Fundamental Computing Structures: Butterflies ......... 18

1-D Data Sequence: Linear and Digit-Reverse .......... 21

Inverse Fast Fourier Transform $\ldots \ldots \ldots \ldots \ldots \ldots \ldots 24$

Two-Dimensional Fast Fourier Transform . . . . . . . . . . 24 
2-D Data Sequence: Linear and Digit Reverse.......... 25

Multi-Dimensional Fast Fourier Transform . . . . . . . . . 27

Discrete Cosine Transform by FFT .............. 28

Method 1: N-Point DCT by Zero Extended 2N-Point FFT . . . . 29

Method 2: N-point DCT by Symmetric Extended 2N-Point

FFT. ........................... 30

Method 3: N-Point DCT by N-Point FFT $\ldots \ldots \ldots \ldots \ldots \ldots . \ldots 31$

III BRIEF DESCRIPTIONS OF SHARP LH9124 AND LH9320. . . . . 34

Design Considerations Of LH9124............... 37

Algorithm: ....................... 37

Arithmetic:....................... 38

Data Path Structure.$\ldots \ldots \ldots \ldots \ldots \ldots \ldots \ldots$

Instructions: . . . . . . . . . . . . . . 39

System:...................... 40

Unified Signal Flow Graph Structure for 1-D to M-D FFT . . . . 42

Block Floating-point Arithmetic and Implementation ....... 46

IV UNIFIED INDEXING OF FFT ALGORITHMS ............ 49

1-D Bit-reverse Inputs And Linear Outputs FFT ........ 50

1-D Linear Inputs And Bit-reverse Outputs FFT . . . . . . . 53

Equivalence of FFT Algorithms by Matrix Transformation .... 54

A. Example of Deriving Constant Geometry FFT from

In-place FFT $\ldots \ldots \ldots \ldots \ldots \ldots \ldots \ldots \ldots \ldots$ 
B. Example of Deriving In-place LI/BO FFT from

In-place BI/LO FFT $\ldots \ldots \ldots \ldots \ldots \ldots \ldots \ldots 56$

Formulation of Vector Matrix Form of 2-D DFT ........ 56

2-D Linear Inputs And Bit_reverse Outputs FFT. ......... 57

2-D Bit-reverse Inputs And Linear Outputs FFT . . . . . . . 59

V WINDSP: A BLOCK ORIENTED WINDOW-BASED DSP

ENVIROMENT .......................... 64

The WinDsP Model . ...................... 66

Equivalence Relationship of the Function-level

Representation $\ldots \ldots \ldots \ldots \ldots \ldots \ldots \ldots \ldots \ldots \ldots \ldots \ldots \ldots$

WinDSP Function-Level Representation Mapping ........ 75

Example of 2-D FFT Application. . . . . . . . . . 79

VI EVALUATION OF RESULTS AND PERFORMANCE

COMPARISON $\ldots \ldots \ldots \ldots \ldots \ldots \ldots \ldots \ldots \ldots \ldots \ldots \ldots \ldots \ldots \ldots \ldots$

Computational Complexity Model ............... 84

Multi-Dimensional FFT Applications . . . . . . . . . . 89

VII CONCLUSIONS AND FUTURE WORK $\ldots \ldots \ldots \ldots \ldots \ldots . \ldots 1$

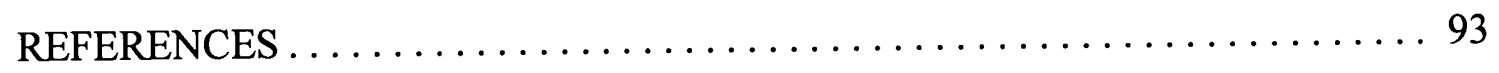

APPENDICES

A ANALYSIS OF UNIFIED INDEXING FOR 1-D TO M-D ........ 96

Computation of 1-D BI/LO 16-point FFT Vector Matrix ..... 98 
B IMPLEMENTATION OF UNIFIED INDEXING TECHNIQUE ON A 2-D 4 BY 4 BI/LO FFT VECTOR MATRIX ........ 103 Computation of 2-D 4 by 4 BI/LO FFT Vector Matrix ....... 104

C TABLE OF UNIFIED INDEXING SEQUENCES FOR PERMUTATION MATRICES. . . . . . . . . ....... 109

D PROGRAM SOURCE CODE OF WINDSP GRAPHICS USER INTERFACE AND FUNCTIONAL BEHAVIORS OF LH9124 INSTRUCTION SET . . . 


\section{LIST OF TABLES}

TABLE

PAGE

I Relation between Linear and Digit-Reverse Order ........... 23

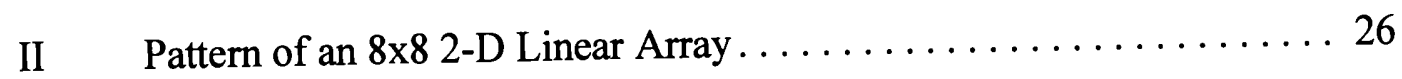

III Pattern of 2-D Array After 1-D FFT for Each Row $\ldots \ldots \ldots \ldots \ldots 26$

IV Pattern of 2-D Array After 2-D FFT .................. 27

V The LH9124 Instruction Set ..................... 35

VI The LH9320 Address Generator Instruction Set for FFT . . . . . . 36

VII Two FFT Examples using Radix-2 with LH9124/LH9320

Instructions .

VIII Address Patterns for LH9320 Instructions. .............. 74

IX Equivalent Relationship of Unified Indexing and LH9320

Instruction Set. . . . . . . . . . . . . . . . 74

$\mathrm{X} \quad$ Spectrum Sharpening of a $1 \mathrm{~K}$ by $1 \mathrm{~K} 2$-D Image........... 80

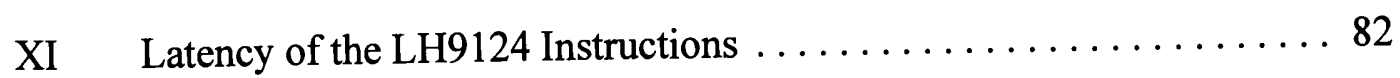

XII Performance Comparison of 1024-point FFT Structures ........ 83

XIII Two FFT Examples using Radix-4 with LH9124/LH9320

Instructions $\ldots \ldots \ldots \ldots \ldots \ldots \ldots \ldots \ldots \ldots \ldots \ldots$

XIV Benchmark of Unified Indexing FFT Algorithm of Different

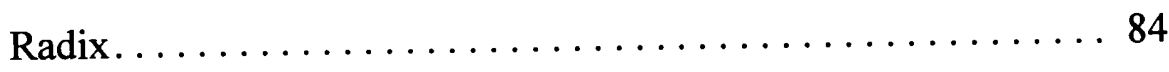


XV Benchmark of Unified Indexing FFT Algorithm using Radix-2 . . . . 86

XVI Benchmark of M-D FFT Applications on LH9124/LH9320 . . . . . 89

XVII Addressing Sequences of 4 by $4 \&$ 16-points 1-D BI/LO FFTs . . . 110 


\section{LIST OF FIGURES}

FIGURE

PAGE

1. Discrete signal $\mathrm{x}$ with independent variable $\mathrm{nT} \ldots \ldots \ldots \ldots \ldots \ldots$

2. The unit circle in the complex $z$-plan $\ldots \ldots \ldots \ldots \ldots \ldots \ldots \ldots$

3. A 8-point DFT decomposed into 8-point 3-stage Radix-2 FFT . . . . 16

4. A flow graph of 8-point FFT with 3-stage DIT Radix-2 Structure . . . . 17

5. Radix-2 Butterfly Structure $\ldots \ldots \ldots \ldots \ldots \ldots \ldots \ldots \ldots$

6. A Flow graph of 8-point FFT with Linear Input and Bit-Reverse

Output

7. A flow graph of Digit-Reverse Input and Linear Output for $4 \times 2$

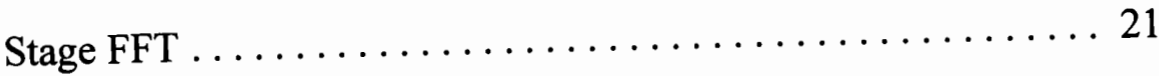

8. N-Point DCT by Zero Extended 2N-Point FFT. . . . . . . . . 30

9. N-Point DCT by Symmetric Extended 2N-Point FFT. . . . . . . 31

10. N-Point DCT of the $\mathrm{N}$ Real Data. .................. 33

11. LH9124 Detailed Block Diagram and Execution Unit. . . . . . . . . 41

12. Signal Flow Graph for 4 by 4 2-D FFT and 16-point 1-D FFT . . . . . 45

13. Signal Flow Graph for Traditional Implementation of 4 by 4

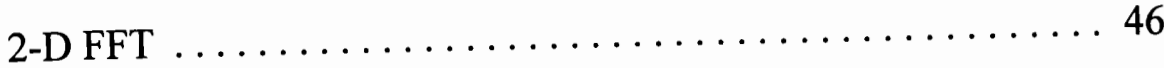

14. Signal Flow Graph of Traditional LI/BO 4 by 4 2-D FFT . . . . . . . 60 
15. Signal Flow Graph of LI/BO 4 by 4 2-D Mapped \& 16-point

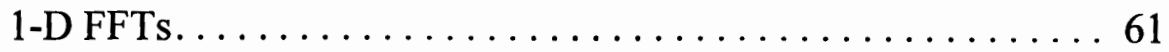

16. Signal Flow Graph of Traditional BI/LO 4 by 4 2-D FFT $\ldots \ldots \ldots 62$

17. Signal Flow Graph of BI/LO 4 by 4 2-D Mapped \& 16-point

1-D FFTs............................. 63

18. Graphical Icons of WinDSP Software ................. 67

19. The WinDSP Software Architecture $\ldots \ldots \ldots \ldots \ldots \ldots \ldots \ldots 68$

20. LH9320 Simulator Memory Map Menu $\ldots \ldots \ldots \ldots \ldots \ldots \ldots$

21. Function Block Diagram of 1-D 16-point \& 2-D 4 by 4 FFT

Computation .......................... 75

22. WinDSP Signal Generator Program Window $\ldots \ldots \ldots \ldots \ldots \ldots$

23. WinDSP Signal Configuration Window $\ldots \ldots \ldots \ldots \ldots \ldots \ldots \ldots$

24. WinDSP Port Display Window $\ldots \ldots \ldots \ldots \ldots \ldots \ldots \ldots \ldots$

25. Block Diagram of $1 \mathrm{~K}$ by $1 \mathrm{~K}$ Image Spectrum Sharpening . . . . . . 79

26. Graph of Actual Computation Cycles vs. Data Size on

LH9124/LH9320........................ 87

27. Graph of Actual Computation Time vs. Data Size on

LH9124/LH9320 . . . . . . . . . . . . . . . . . . . . 88

28. Graph of Number of FFT per second vs. Data Size on

LH9124/LH9320....................... 88 


\section{CHAPTER I}

\section{INTRODUCTION}

With advances in today's digital signal processing (DSP) algorithms and very large scale integration (VLSI) technologies, array signal processors (ASP) with highly parallel computing structures have become increasingly important in recent years [Haykin, 1985]. By definition, parallel computing structures imply a basic computational element repeated perhaps hundreds or thousands of times [Hwang and Briggs, 1984]. Array signal processing deals with propagating signals received by an array of sensors (or a multichannel of sensors). Array signal processors handle large volumes of data from multi-channel arrays. Traditional analog signal processing can provide the required speed. However, strict stability (i.e. requiring fine adjustment) and resolution requirements (mathematics finess) are difficult to obtain. Therefore, the design of an array digital signal processor chip architecture, the development of DSP algorithms and the implementation of the system are solutions to analog problems. The digital system is usually constrained by processing speed and the I/O bottleneck of array processing. Limited sampling rate and complexity of algorithms adopted in the processor are classic examples that degrade system speed and performance.

The increasing performance demands in modern signal and image processing applications necessitate a revolution in DSP algorithms and super-computing technology. The Fast Fourier Transform (FFT) is usually a fundamental driving force for real-time 
digital signal processing in array processor systems such as radar, sonar, seismic, medical image, telecommunication, etc. Array signal processing must handle multi-dimension and multi-channel problems. Array processing performance is usually offset by the steps required for multi-dimension indexing or addressing. With the availability of low-cost, high density and high-speed VLSI devices, the FFT algorithm has pushed multidimensional array signal processing into real-time. However, there is no efficient addressing method for one dimensional (1-D) to multi-dimensional (M-D) FFTs. A unified indexing technique developed and defined by C. J. Ju [Ju, 1993] solved the problems of addressing method for 1-D to M-D FFTs. This technique has simplified all multidimensional indexing. But the question remains: How well is the unified indexing technique mapped to the DSP architecture of the commercial chip set? What advantages does the unified addressing technique have over the traditional method? To be feasible, the DSP chip set must be implemented within reasonable bounds of hardware requirements and constraints, yet perform with real-time efficacy. The Sharp LH9124/ LH9320 chip set is of particular interest, relative to the contemporary FFT processors because of its highly parallel and pipeline architecture and its by-pass form execution unit.

This thesis involves the implementation of unified indexing of FFT algorithms and shows the equivalent relationship between function-level representation of the LH9124 FFT instructions and the vector matrices transformed using the unified indexing approach. These led to the development of WinDSP software simulator which implements the unified indexing concepts and simulates the functional characteristics of the LH9124/ LH9320 chip set. An overview of fundamental digital signals, a brief description of 
LH9124/LH9320 architecture and a derivation of the unified addressing FFT algorithm are given to show constraints and conditions imposed on the design of the WinDSP software simulator.

\section{ALGORITHM ANALYSIS, DERIVATION AND MAPPING}

Most array signal processors are special-purpose DSP chips. Therefore, the architecture design is application-driven or algorithm-driven. The algorithm development and algorithm-to-chip architecture mapping are essential to the overall system performance. The algorithm is analyzed to get special features and to find efficient solutions for a specific set of problems. Our applications are in array signal processing. Some features are specific to those applications. First, they are compute-intensive applications. Second, they usually have to handle multi-dimension and multi-channel problems. Third, they encounter scaling problems due to the block floating-point arithmetic. Finally, the applications must be configured into parallel or pipeline architectures to meet real time speed requirements such as latency and throughput.

To conquer compute-intensive and highly (or mostly) parallel applications, FFTbased block-oriented DSP algorithms will be adopted. The multi-dimensional (M-D) Fast Fourier Transform is an essential algorithm for most applications. In M-D FFT computations, the performance of enhanced array processor architecture structures is greatly degraded by the M-D indexing calculations. Moreover, the signal reference level adjustments (scaling) required in M-D FFT computations also make array processors quite inefficient compared to the one-dimensional case. These two problems must be 
solved to design efficient array systems.

To support efficient computations of the M-D FFT, a novel unified indexing FFT algorithm was developed [Ju, 1993]. Unified indexing means that the general M-D FFT algorithm indexing can be mapped to 1-D FFT indexing and can be computed by 1-D FFT method. These including data, twiddle factors, and digit-reverse addressing. The algorithm derivation can be described in the following.

\section{1-D EET Derivation and Analysis}

It is well-known that the computing efficiency of the FFT comes from recursive factorization of the twiddle factor matrix in the Discrete Fourier Transform (DFT) [Oppenheim and Schafer, 1989]. The matrix can be recursively partitioned and factored into butterfly stage matrices. Each butterfly stage matrix can be further factored into three matrices: A right permutation matrix, a central butterfly computation matrix, and left permutation matrix. The three-matrix representation of the butterfly stage matrix has essential meaning in the physical FFT algorithm implementation [Ju, 1993]. The right permutation matrix can represent the input interconnection and define the input data address sequence of the stage. Similarly, the left permutation matrix can denote the output interconnection and also define the output data address sequence of the stage. The data address sequence including the digit-reverse address sequence can be directly obtained from the locations of ones from top to bottom of the permutation matrix. The central butterfly computation matrix is a block diagonal matrix which performs the butterfly operations of the stage. This matrix can also be used to derive the twiddle factor address sequence. 


\section{Unified Indexing for M-D FETs}

As in the 1-D case, the twiddle factor matrix of the M-D Discrete Fourier Transform (DFT) can be decomposed into the multiplications of the butterfly stage matrices, see "Fundamental Computing Structures: Butterflies" on page 18. It can be derived through equivalent matrix transformations since the M-D FFT has the same matrix structure as the 1-D FFT when both have the same number of data, see "Unified Signal Flow Graph Structure for 1-D to M-D FFT" on page 42. In addition, the permutation matrices for both 1-D and M-D FFTs in each stage are the same. This implies that the signal flow graph structure of the M-D FFT can be mapped to that of the 1-D FFT. Thus, the data and digit-reverse indexing of the 1-D FFT can be universally applied to those of the M-D FFTs. It can also be shown that the twiddle factor matrices of the 1-D FFT are the subset of those of the M-D FFT. This means the twiddle factor sequence of the 1-D FFT can also be used for that of the M-D FFT.

\section{PROBLEM STATEMENT}

The array processor LH9124 and address generator LH9320 developed by Sharp Microelectronics Technology [LH9124 and LH9320 User's Guide] are high speed chips and at the time of their introduction were innovative designs for real-time applications. When it's used for processing large data set in complex system applications, they still ran into system architecture problems. Systems with large number of data mostly want to process multidimension and multi-channel FFTs. Thus, to implement a M-D FFT algorithm, the traditional method required many instructions and excessive pipeline 
overhead to compute the data. For example, in the traditional method for 2-D FFT, each individual row vector is computed and the scaling factor extracted. Then each individual row is scaled with the maximum scaling factor extracted. This same process is applied for each column vector. Thus, the whole process will takes excessive hardware and software to accomplish the FFT computation. There is no efficient addressing method for 1-D to M-D FFTs. Hence, the unified indexing for 1-D to M-D approach was proposed to address these problems.

There are several advantages for implementing the unified indexing technique. First, the number of FFT instructions required is greatly reduced. Less physical memory is used and the performance can be improved by reducing instruction pipelined overhead. For example, the unified approach requires only 3 instructions to implement 16 by 16 by $163-\mathrm{D}$ FFT, while the traditional approach requires 768 instructions. Second, no data matrix transposition is required because the transposition is already covered in the M-D to 1-D Signal Flow Graph structure mapping. Thus it is much easier for users to implement the M-D FFT without considering the time-consuming complex matrix transposition. Third, the unified approach automatically solves the scaling problems that occur in the block floating-point arithmetic. The traditional approach requires extra hardware or software to solve the problem. Fourth, the simplified and unified 1-D addressing not only speeds up the M-D FFT algorithms but also makes the chip function definition easier. Finally, the function instructions of LH9124 reduces effort in program coding and debugging. 


\section{GOALS AND DESIGN DEVELOPMENT METHODOLOGY}

Starting from the vector-matrix representation, the equivalent relationship between 1-D and M-D FFTs employing the equivalent transformation of the matrices has been defined in this thesis. Particularly, it will be shown that the signal flow graph (SFG) structure of the 1-D FFT can be used to represent that of the M-D FFT regardless of the dimension if the total number of elements is the same. The radix-2 FFT is the basis of all these implementations. The novel unified indexing for 1-D to M-D FFT algorithms can be implemented in the LH9124/LH9320 FFT architecture. Not all FFT chip set architectures can implement the unified indexing technique.

The primary goal of the thesis is to implement the unified indexing methodology in WinDSP software simulator for 1-D to M-D FFT alogrithm. The window-based software simulator not only simulates the functional characteristics of the LH9124/ LH9320 chip set, but also presents the function block diagram equivalent of functionlevel representation and the unified indexing matrix. WinDSP software simulator can be used to verify the correctness and performance of M-D FFT applications that using the concept of novel unified indexing vector matrix compared with the traditional method. WinDSP was developed solely by the author and has been evaluated by commercial companies like G. E. Medical, Catalina Research, Baka Corporation and etc. WinDSP software tool can be used to verify the unified indexing implementation for 1-D to M-D FFT in near real-time computation. With the implementation of the LH9124/LH9320 DSP chip set, it can be shown that the unified approach for M-D FFT has tremendous advantage over the traditional M-D FFT approach in performance. Another advantage 
expected is there will be a cost saving in hardware and software development. This approach can be extended to the fast cosine transform adopted by image processing systems that require fast high-end real-time computations.

\section{ORGANIZATION OF THIS THESIS}

In Chapter I, a brief introduction to system requirements and specification for fast array processors is given. FFT algorithm analysis, derivation and mapping are also briefly outlined. The important aspect of this thesis is to implement the unified indexing approach for 1-D to M-D FFT using WinDSP simulator as a tool. The function-level representation mapping between the unified indexing of the FFT algorithm and the LH9124 FFT instruction set will also be presented in this thesis by the author.

WinDSP simulates the function and the data flow characteristics of the LH9124. Each function block diagram of the WinDSP is also the function-level representation of an instruction in the LH9124. The mapping relationship of WinDSP and unified indexing approach of the FFT will be introduced. The function block diagram is object-oriented, has clear visualization, allows conceptualization and prototyping, and is easy for real-time hardware implementation.

In Chapter II, a brief overview of the Fast Fourier Transform (FFT) and Fast Cosine Transform (FCT) algorithm is given. The derivation of FFT from DFT will be discussed, the derivation of FCT and three ways of implementing the FCT on the LH9124 will also be introduced, together with applications, using the concept of Unified Index Addressing. 
Chapter III briefly introduces the LH9124 chip architecture and design considerations. Again some mathematical derivations, analysis and mappings will be presented.

Chapter IV presents the concept of the Unified Index Addressing of FFT. The detailed algorithms analysis, mathematical derivations and data-flow-graph mappings of the FFT indexing will be presented. Following that, the concept of mapping 1-D FFT to M-D addressing will be explained and its simplicity will be emphasized. The architectural and operational description of the FFT data I/O design will also be provided.

Chapter V is devoted to the software WinDSP, a function block and system block level simulation. The description of designing the software environment that manages a complete application development from the conceptualization stage will be given. The mapping relationship between the unified indexing matrix and the function block diagram of the WinDSP will be presented. The importance of this tool is that it helps to understand the mapping relationships between the algorithmic flow graph structure and its index addressing. Some examples will show the direct relationship of the flow-graph indexing using the user-friendly simulator for designing applications at the fundamental level.

In Chapter VI, the evaluation and performance of the FFT examples are analyzed and compared to competing approaches. The benchmark for implementing unified indexing approach and the traditional approach will be analyzed.

Chapter VII closes the thesis with concluding remarks and summarizes the achievements of the thesis.

Appendix A contains the detailed examples of 1-D 16-point implementations of 
the unified indexing algorithm. Appendix B shows the example of using unified indexing on 4 by 4 2-D FFT algorithms. Appendix $C$ presents the address sequence in a table from examples in Appendix A and B. Finally, Appendix D lists all the source code of the WinDSP simulation program. 


\section{CHAPTER II}

\section{FUNDAMENTALS OF DIGITAL SIGNALS}

A signal can be defined as a function that conveys information. Generally, a signal is about the state or behavior of a physical system. Signal can be represented in many different ways, but in most cases, the information contained in the signal can be mathematically analyzed. Signals can be represented as functions of one or more independent variables. For example, an image signal is represented mathematically on brightness of two spatial variables, whereas the speech signal is represented as a function of one independent variable. The independent variables in the mathematical representation of a signal may be continuous or discrete. Continuous-time signals are often referred to as analog signals, and are defined along a continuum of time. Discretetime signals are represented as sequences of numbers and are defined at discrete times. In DSP applications, a sequence of numbers $x$, in which the $n$-th number in the sequence is denoted $x[n]$, is formally written as

$$
x=\{x[n]\}, \quad n=\{\ldots,-4,-3,-2,-1,0,1,2,3,4, \ldots\}
$$

where $n$ is an integer. In a practical setting, such sequences can often arise from periodic sampling of an analog signal. In this case, the numeric value of the $n$-th number in the sequence is equal to the value of the analog signal $x_{a}(t)$ at time $n T$; i.e.

$$
x[n]=x_{d}(n T), \quad n=\{\ldots,-4,-3,-2,-1,0,1,2,4, \ldots\}
$$


where $T$ is the uniform interval (typically a time interval) between input samples. $x_{a}(n T)$ can represent a sequence of numbers generated on a computer, data taken from an experiment or numbers from some natural phenomena that have been sampled. For simplicity, the sequence is denoted as $x(n T)$ or $x(n)$, where the uniform sampling period is implied. A discrete sequence is shown in Figure 1.

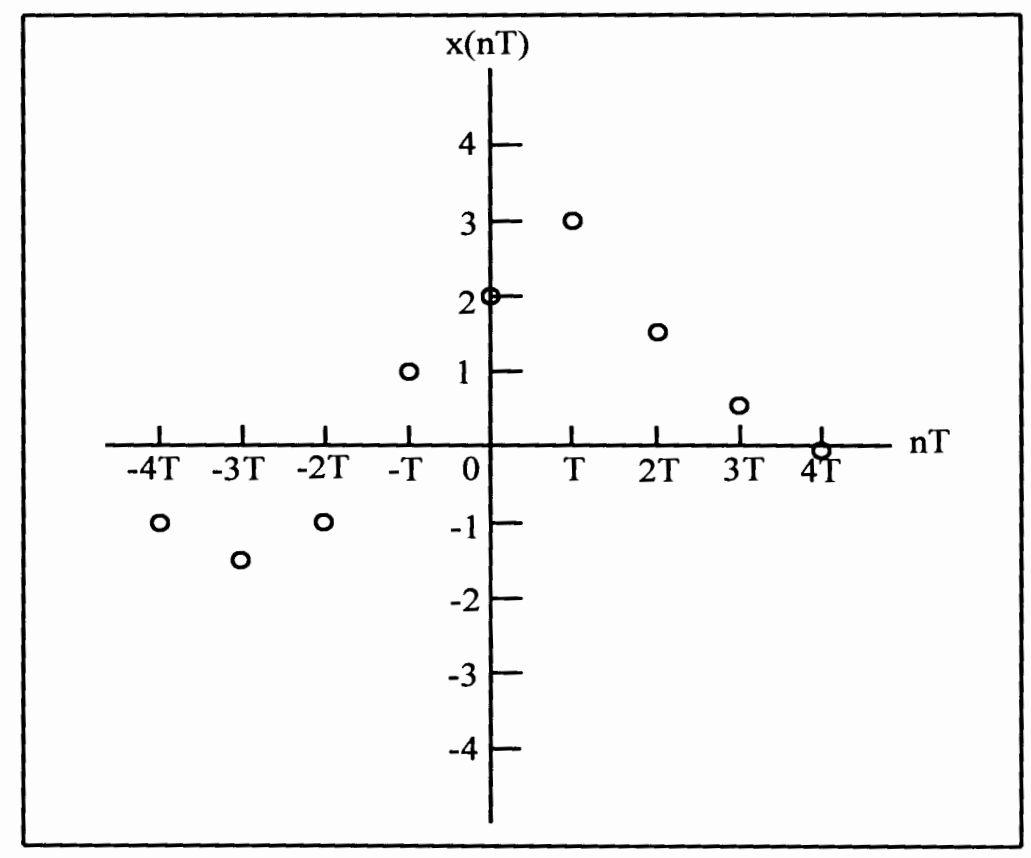

Eigure 1. Discrete signal $\mathrm{x}$ with independent variable $\mathrm{nT}$

In many applications, the class of signals of interest is periodic. The periodic class of signals has an efficient method of analyzing the signal. The Fourier transform is commonly used to obtain the frequency spectrum of the signal. The frequency spectrum of a signal shows its periodic character.

Signal conveys specific information that the DSP system is attempting to interpret. One goal of a DSP system is to provide the best approach to analyzing or estimating the information content of the signal. 


\section{Overview of Fast Fourier Transform}

The Discrete Fourier Transform (DFT) plays an important role in the analysis, design, and implementation of discrete-time signal processing algorithms and systems. The DFT is the link between the time representation $x(n)$ and the frequency representation $X(k)$. The DFT is a sequence rather than a function of a continuous variable, and it corresponds to the equally-spaced frequency-sampled Fourier transform signal. The Fourier transform has been a powerful and principal analysis tool in diverse fields such as linear systems, probability theory, boundary-valued problems, communications theory, signal processing and many other. For instance, general applications for Fourier transform algorithm include; magnetic resonance imaging (MRI), computer-assisted tomography (CAT) scanners, radar system, telecommunications and etc.

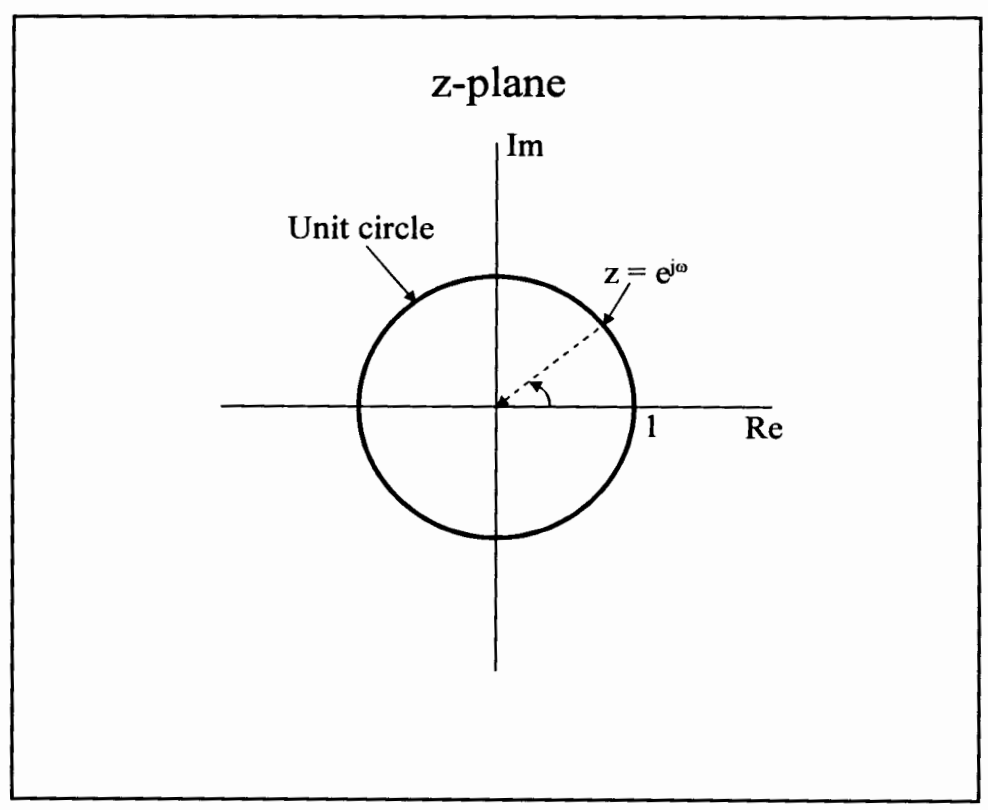

Eigure 2. The unit circle in the complex $z$-plane

The definition of the DFT is given by the expression: 


$$
X(k)=\sum_{n=0}^{N-1} x(n) W_{N}^{k n} \text { for } k=0,1, \ldots ., N-1
$$

and the inverse of DFT, IDFT, is expressed as:

$$
x(n)=\frac{1}{N} \sum_{k=0}^{N-1} X(k) W_{N}^{-k n} \text { for } n=0,1, \ldots . ., N-1
$$

where $W_{N}^{k}=e^{-j 2 \pi(k / N)}$ is a sequence of twiddle factors of the DFT and is equally spaced around the unit circle in the complex plane. The unit circle is defined as the contour of a circle, in $z$-plane, correspond to $|z|=1$ unit radius, as illustrated in Figure 2. In these equations $x(n)$ is the sample value in the time domain and $X(k)$ is the sample value in the frequency domain. If the sampling rate of a signal is $\boldsymbol{F}$, the sequence of timedomain sampling locations becomes

$$
0,1 / F, 2 / F, 3 / F, \ldots,(N-1) / F
$$

Thus, corresponding to these time-domain samples, the sequence of frequencydomain sampling locations will be

$$
0, F / N, 2 F / N, 3 F / N, \ldots,(N-1) F / N
$$

The computation of the DFT and IDFT is basically a vector dot operation. The DFT and IDFT both produce periodic results with period N [Oppenheim and Schafer, 1989]. It can be seen from Equations (2.3) and (2.4) that some symmetric properties also exist. A direct computation of the DFT or IDFT requires $N^{2}$ complex multiplications and $N(N-1)$ complex additions. The FFT is an efficient algorithm for computing the DFT and can be derived from the DFT. The discrete Fourier transform can be computed in $O(N$ $\log _{2} N$ ) complex multiplication operations by using the FFT algorithm. 


\section{Formation of the FFT}

The Fast Fourier Transform (FFT) algorithm achieves its computational efficiency through a divide and conquer strategy. The essential idea is a grouping of the time and frequency samples such that the DFT summation over $\mathrm{N}$ values can be expressed as a combination of DFT summations over $\mathrm{N} / 2$ samples. When $\mathrm{N}$ is a power of two, this process of grouping can be repeatedly applied until the DFT summation has been reduced to a combination of DFT summation over only two samples. For example, Equation (2.3) can be divided as follows when $\mathrm{N}$ is a power of two.

$$
\begin{aligned}
X(k) & =\sum_{n=0}^{N / 2-1} X(2 n) W_{N}^{2 n k}+\sum_{n=0}^{N / 2-1} x(2 n+1) W_{N}^{(2 n+1) k} \\
& =\sum_{n=0}^{N / 2-1} x(2 n) W_{N}^{2 n k}+W_{N}^{k} \sum_{n=0}^{N / 2-1} x(2 n+1) W_{N}^{2 n k}
\end{aligned}
$$

Define two (N/2)-point sequences $\{h(n)\}$ and $\{g(n)\}$ as the even and odd elements of $\{x(n)\}$ respectively. Then,

$$
\begin{gathered}
h(n)=x(2 n) \\
g(n)=x(2 n+1)
\end{gathered}
$$

The discrete Fourier transform of the two (N/2)-point sequences can be defined as follows

$$
\begin{aligned}
& H(k)=\sum_{n=0}^{N / 2-1} h(n) W_{N / 2}^{k n} \\
& G(k)=\sum_{n=0}^{N / 2-1} g(n) W_{N / 2}^{k n}
\end{aligned}
$$

Thus, the discrete Fourier transform $\{x(n)\}$ can be expressed in terms of even and odd 
elements as

$$
X(k)=\sum_{n=0}^{N / 2-1} h(n) W_{N / 2}^{2 n k}+\sum_{n=0}^{N / 2-1} g(n) W_{N / 2}^{2 n k}
$$

In terms of $H(k)$ and $G(k)$, we have

$$
X(k)=H(k)+W_{N}^{k} G(k)
$$

For the coefficient at $(k+N / 2)$, we obtain

$$
X(k+N / 2)=H(k)-W_{N}^{k} G(k)
$$

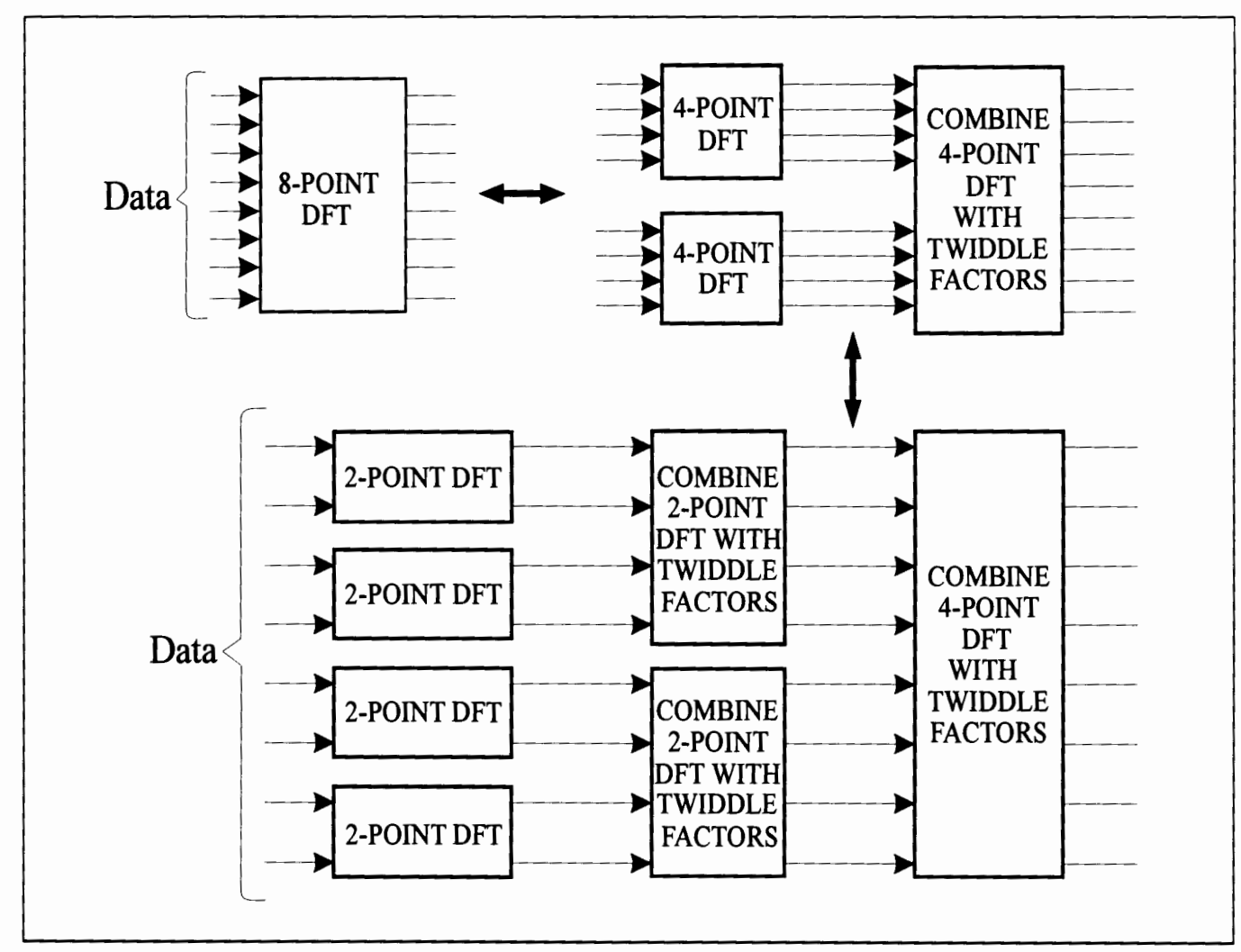

Eigure 3. A 8-point DFT decomposed into 8-point 3-stage Radix-2 FFT

Therefore, N-Point discrete Fourier transform can be represented by two (N/2)- 
point discrete Fourier transforms. Since $\mathrm{N}$ is a power of two, the above partitioning scheme can be iteratively applied to the sequences $\{h(n)\}$ and $\{g(n)\}$ by N/4 elements. These partitions can be carried out until the two-point DFT is reached. The process is depicted in Figure 3 for $N=8$. Figure 4 shows the flow graph of an 8-point FFT that denotes the results of the 8-point decomposed DFT.

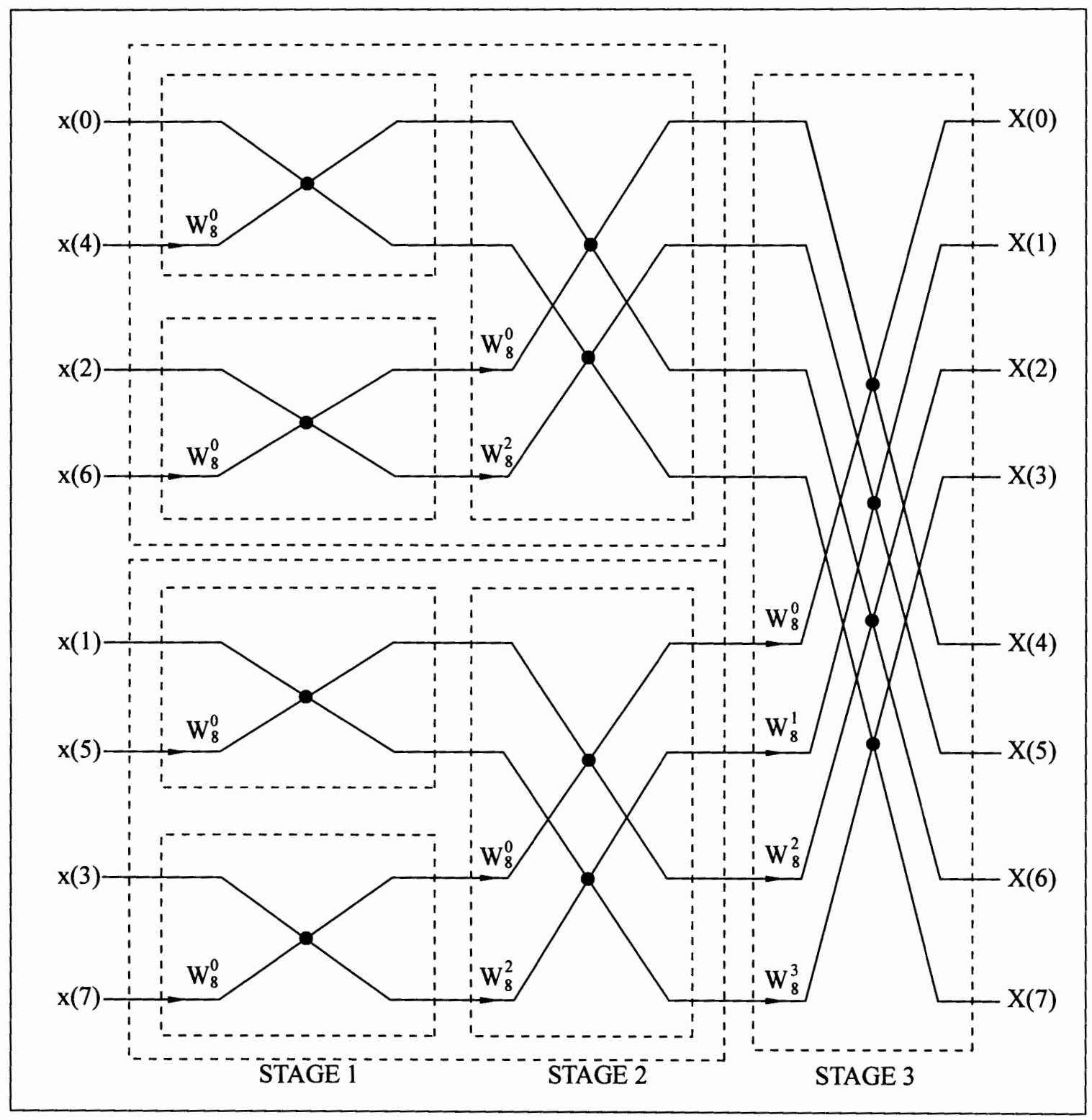

Eigure 4. A flow graph of 8-point FFT with 3-stage DIT Radix-2 Structure 


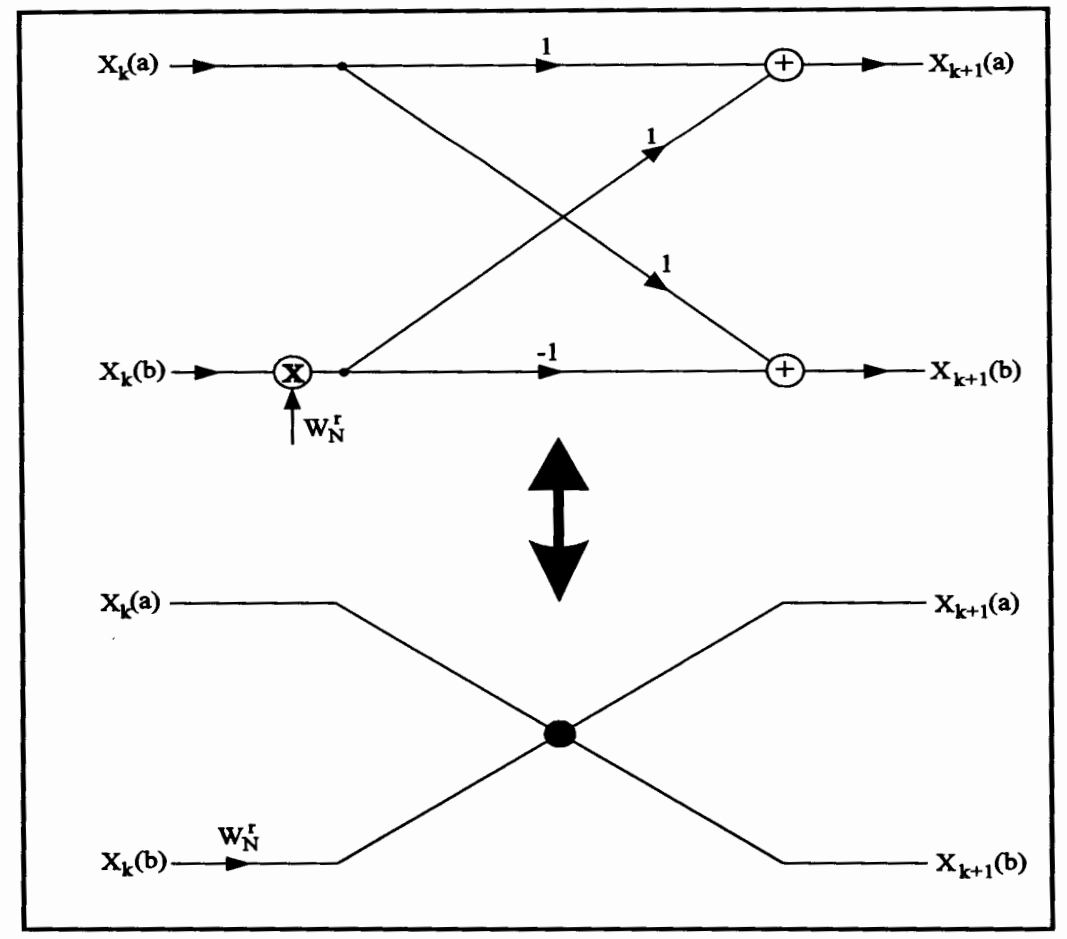

Figure 5. Radix-2 Butterfly Structure

\section{Fundamental Computing Structures: Butterflies}

The structure shown in Figure 4 is called decimation-in-time (DIT). Its basic module is a radix-2 butterfly shown in Figure 5 in which two points $x_{k}(a)$ and $x_{k}(b)$ are computed to give two output points $x_{k+1}(a)$ and $x_{k+1}(b)$ via the operations represented by Equations (2.13) and (2.14). Because of the shape of the flow graph, this elementary computation is called a butterfly. Each radix-2 butterfly requires one complex multiplication and two complex additions, as illustrated in Figure 5 and where the twiddle factors (coefficients) are always powers of $\mathrm{W}_{\mathrm{N}}$ and the exponents are separated by $\mathrm{N} / 2$. Observing Figure 4, it can be seen that there $\operatorname{are} \log _{2} \mathrm{~N}$ radix-2 butterflies. Therefore, there are a total of $(\mathrm{N} / 2) \log _{2} \mathrm{~N}$ radix-2 butterflies in an N-point FFT. In addition, the input is in the bit-reverse order and the output is in the linear order. Further explanation of the 
terms "bit-reverse order" and "linear order" can be seen under the section: "1-D Data Sequence: Linear and Digit-reverse" on page 21.

From the flow graph shown in Figure 4, some other structures of FFT can be derived by exchanging the indexing of the flow graph. One of these structures is shown in Figure 6, where the input indexing (or addressing) is in the linear order and the output indexing is in the bit-reverse order. Both structures employ the in-place algorithm. Inplace means the computed outputs can be placed in the same memory location (storage) as the inputs. Moreover, the addressing for the input and output data can be shared. This is important because the addressing for that stage data is generated only once. One more structure equivalent to the structure from Figure 4 is depicted in Figure 7. The four radix2 butterflies enclosed in the dashed box can be replaced with a radix -4 butterfly. The structure has inputs in digit-reversed order and outputs in linear order. It is not an in-place algorithm for radix-2 but it is an in-place algorithm for radix-4.

The N-point FFT can be implemented by the mixed radix operation as long as the following relation exists

$$
N=r_{0} \times r_{1} \times r_{2} \times \ldots \times r_{k-1}
$$

where $r_{i}$ is 2 for a radix- 2 stage, 4 for a radix -4 stage and 16 for a radix-16 stage. The $r_{0}$ is the first stage and $r_{k-1}$ is the last stage of the FFT. The order of the stages can be arbitrarily selected when implemented by the chip set. If $N=2^{M}$, there are M stages by the radix-2 structure. Then the FFT can be defined to have $\mathrm{M}$ columns. For an arbitrary j-th column, they are three ways to implement the column. First, it may be directly implemented as a radix- 2 stage. Second, it and its next column may be implemented by a radix-4 stage. 
Third, it and its next three columns may be implemented by a radix-16 stage.

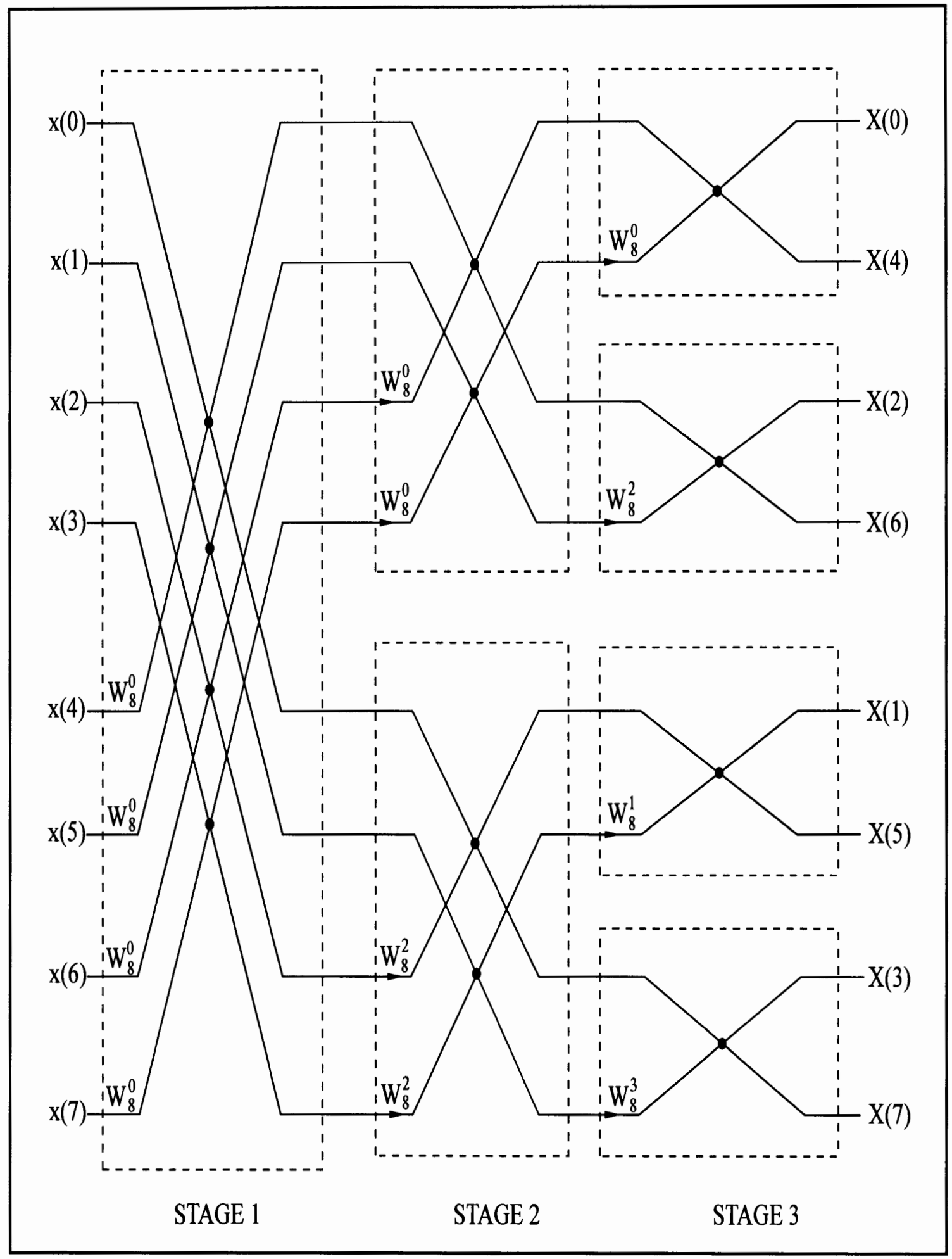

Eigure 6. A Flow graph of 8-point FFT with Linear Input and Bit-Reverse Output 


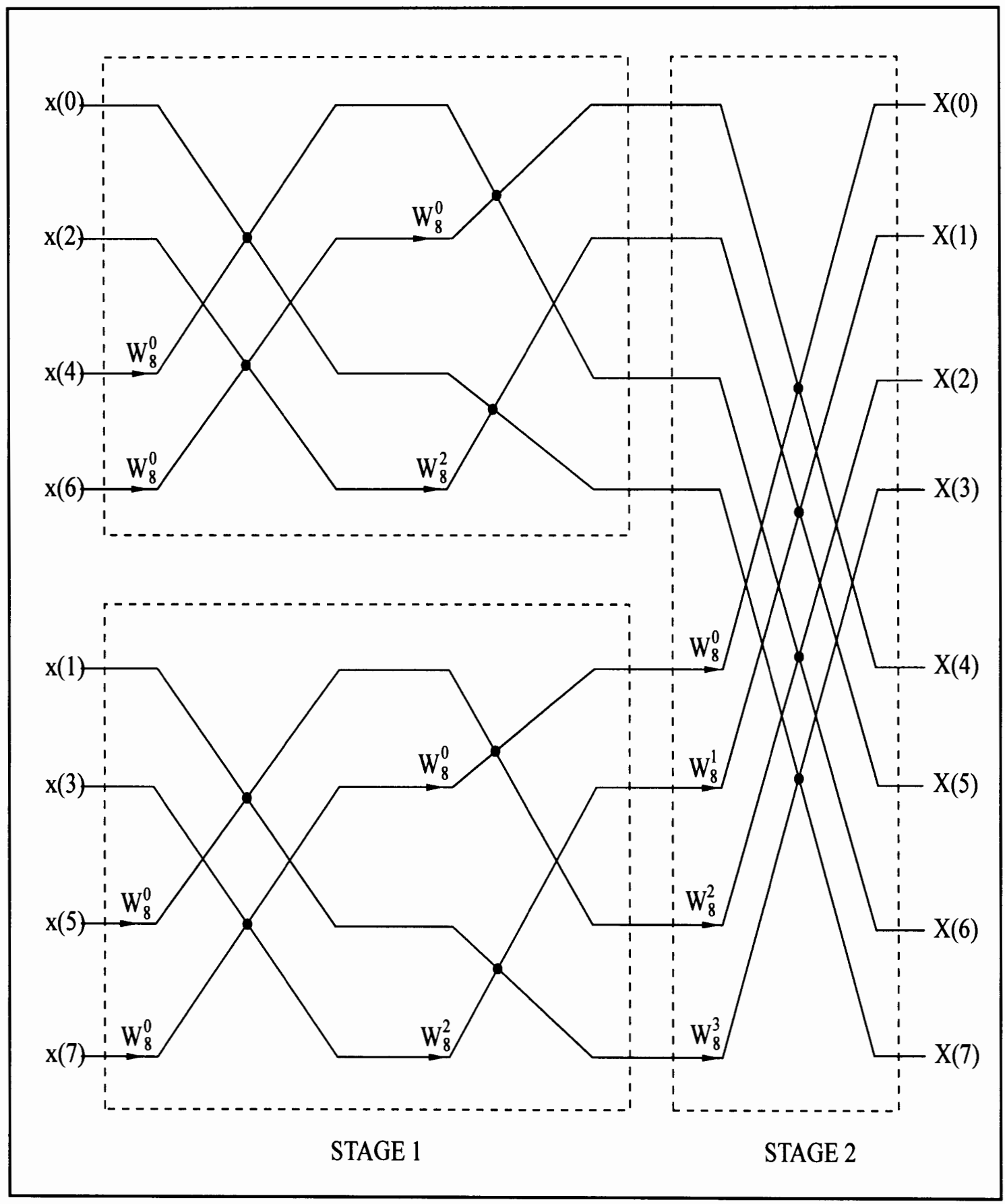

Eigure 7 A flow graph of Digit-Reverse Input and Linear Output for 4x2 Stage FFT

\section{1-D Data Sequence: Linear and Digit-reverse}

The decimation-in-time FFT algorithm with inputs in digit-reverse order and 
outputs in linear order is implemented in Sharp chip set. Set a digit number $n=n_{N-1} n_{N-2} \ldots$ $n_{l} n_{0}$ and assume the radix of $n_{i}$ is $r_{i}$. For example, a 16-point FFT using radices $\left(r_{i}\right)$ of $2 \times 2 \times 4$, the digit number $n=013$ (of mixed radix) starting from right to left translates to $\left(2^{1} 4^{1}\right)^{*} 0+\left(2^{0} 4^{1}\right)^{*} 1+\left(4^{0}\right)^{*} 3=\left(8^{*} 0\right)+\left(4^{*} 1\right)+\left(1^{*} 3\right)=7_{10}($ decimal number $)$. A binary number is a special case of a digit number with the radius of all the $n_{i}$ being 2 . The digit number implemented by the Sharp chip set for the FFT has the constraint that the radix of an arbitrary $n_{i}$ is 2 or 4 . A digit number used by the radix-16 is actually represented by two digits $n_{i} n_{i-1}$ with radix of each digit being 4 . The weighting factor of $n_{i}$ is obtained by

$$
W_{i}=r_{i-1} \times r_{i-2} \times \ldots \times r_{1} \times r_{0}
$$

The function of digit-reverse is then defined as follows

$$
d r(n)=d r\left(n_{N-1} n_{N-2} \ldots n_{1} n_{0}\right)=n_{0} n_{1} \ldots n_{N-2} n_{N-1}
$$

In this case, from the example above where $n=013$, the digit reverse $d r(n)=310$. If the pattern of its associated radius $r_{N-1} r_{N-2} \ldots r_{l} r_{0}$ is symmetric, the function of digit-reverse is reversible. Thus,

$$
d r(d r(n))=n
$$

The function of bit-reverse is always reversible because its radius pattern is symmetric.

The digit-reverse sequence can be generated in the same way as the linear sequence. Both the sequences can be recursively generated by adding a fixed addend as follows

$$
S_{n+1}=S_{n}+\text { addend }
$$

There are two differences in interpreting the Equation (2.19). First, the carry digit is propagated from right to left for the linear sequence and from left to right for the digit- 
reverse sequence. Second, the addends for the two are different. The addend of the linear sequence is 1 for the least significant digit and 0 for all the other digits. The carry over digit is left propagated. For example in $2 \times 2 \times 2$ structure, $011+1=100$ and in $4 \times 2$ structure, $03+1=10$. The addend of the digit-reverse sequence is 1 for the most significant digit and 0 for all the other digits. The carry over digit is right propagated. For example in $2 \times 2 \times 2$ structure, $110+1=001$ and in $4 \times 2$ structure, $30+1=01$.

Table I: Relation between Linear and Digit-Reverse Order

\begin{tabular}{|c|c|c|c|c|c|c|c|}
\hline \multicolumn{4}{|c|}{$2 \times 2 \times 2$ Structure } & \multicolumn{4}{|c|}{ 2x4 Structure } \\
\hline Line & & Digit & -reverse & Line & & Digit & -Reverse \\
\hline Decimal & Digit & Digit & Decimal & Decimal & Digit & Digit & Decimal \\
\hline 0 & 000 & 000 & 0 & 0 & 00 & 00 & 0 \\
\hline 1 & 001 & 100 & 4 & 1 & 01 & 10 & 2 \\
\hline 2 & 010 & 010 & 2 & 2 & 02 & 20 & 4 \\
\hline 3 & 011 & 110 & 6 & 3 & 03 & 30 & 6 \\
\hline 4 & 100 & 001 & 1 & 4 & 10 & 01 & 1 \\
\hline 5 & 101 & 101 & 5 & 5 & 11 & 11 & 3 \\
\hline 6 & 110 & 011 & 3 & 6 & 12 & 21 & 5 \\
\hline 7 & 111 & 111 & 7 & 7 & 13 & 31 & 7 \\
\hline
\end{tabular}

Both the linear and digit-reverse sequences of 8-points with the radices pattern of $2 \times 2 \times 2$ and $2 \times 4$ are shown in Table I. For the $2 \times 2 \times 2$ radices pattern, the addend is 001 for the linear sequence and 100 for the digit-reverse sequence. For the $2 \times 4$ radices pattern, the addend is 01 for the linear sequence and 10 for the digit-reverse sequence. It can be seen from Table I that the digit-reverse sequence can also be obtained by reflecting the digits according to the center digit. For example, the digit-reverse of 1234 and 12345 are 4321 and 54321, respectively. 


\section{Inverse Fast Fourier Transform}

The inverse FFT (IFFT) can employ the same address patterns as the forward FFT. The equation shown in Equation (2.4) can be expressed by the following form

$$
x(n)=\frac{1}{N}\left[\sum_{n=0}^{N-1} X^{*}(k) W_{N}^{k n}\right]^{*} \quad \text { for } n=0,1, \ldots, N-1
$$

where the notation of the superscript ${ }^{*}$ denotes the conjugated data. If the input frequency samples are conjugated, the bracketed term in Equation (2.20) is exactly an FFT operation. Thus, the computation of the IFFT can share all the instructions of the FFT. It can be seen from Equation (2.20) that the final computed results have to be conjugated also and scaled by the weight of $1 / N$.

\section{Two-Dimensional Fast Fourier Transform}

Considering a complex function defined over a two-dimensional region $0 \leq n_{1} \leq N_{1}-1$ and $0 \leq n_{2} \leq N_{2}-1$, we can define a two-dimensional discrete transform over the same region as follows

$$
\begin{aligned}
X\left(k_{1}, k_{2}\right) & =\sum_{n_{1}=0}^{N_{1}-1} \sum_{n_{2}=0}^{N_{2}-1} x\left(n_{1}, n_{2}\right) W_{N_{1}}^{n_{1} k_{1}} W_{N_{2}}^{n_{2} k_{2}} \\
\text { for } 0 & \leq k_{1} \leq N_{1}-1 \text { and } 0 \leq k_{2} \leq N_{2}-1
\end{aligned}
$$

Equation (2.21) can be reordered into the column-row expression

$$
X\left(k_{1}, k_{2}\right)=\sum_{n_{2}=0}^{N_{2}-1}\left[\sum_{n_{1}=0}^{N_{1}-1} x\left(n_{1}, n_{2}\right) W_{N_{1}}^{n_{1} k_{1}}\right] W_{N_{2}}^{n_{2} k_{2}}
$$


or into the row-column expression

$$
X\left(k_{1}, k_{2}\right)=\sum_{n_{1}=0}^{N_{1}-1}\left[\sum_{n_{2}=0}^{N_{2}-1} x\left(n_{1}, n_{2}\right) W_{N_{2}}^{n_{2} k_{2}}\right] W_{N_{1}}^{n_{1} k_{1}}
$$

If the item in the bracket of Equation (2.22) is expressed by a 2-D function $F\left(k_{1}, n_{2}\right)$, then the 2-D FFT can be computed by two phases of 1-D FFT as follows

$$
\begin{aligned}
& F\left(k_{1}, n_{2}\right)=\sum_{n_{1}=0}^{N_{1}-1} x\left(n_{1}, n_{2}\right) W_{N_{1}}^{n_{1} k_{1}} \text { for } 0 \leq k_{1} \leq N_{1}-1 \\
& X\left(k_{1}, k_{2}\right)=\sum_{n_{2}=0}^{N_{2}-1} F\left(k_{1}, n_{2}\right) W_{N_{2}}^{n_{2} k_{2}} \text { for } 0 \leq k_{2} \leq N_{2}-1
\end{aligned}
$$

Thus, the 2-D FFT can be obtained by computing 1-D FFT for each row of $x\left(n_{l}\right.$, $n_{2}$ ), putting the result into an intermediate array, and then computing 1-D FFT for each column of the intermediate array.

\section{2-D Data Sequence: Linear and Digit Reverse}

In the 1-D case, if the input sequence is in the linear order, the output sequence after FFT operations will be in the digit-reverse order, and vice-versa, according to the FFT Signal Flow Graph operation (refer to Figure 2.4). The 2-D FFT can be obtained by doing two phases of the 1-D FFT for each vector. Therefore, the indices $k_{1}$ and $k_{2}$ of the output array after 2-D FFT are both in digit-reverse order with respect to the indices $n_{l}$ and $n_{2}$, respectively. For example, Table II shows the pattern of a $(8,8) 2$-D input array. Assume that the 8-point column and row are implemented by the 1-D FFT with a radix-4 
stage followed by a radix-2 stage. The function $F\left(k_{1}, n_{2}\right)$ of the intermediate stage 2-D array after row operations is shown in Table III. It can be seen that the index $k_{1}$ is in digitreverse order and $n_{2}$ is in linear order. Table IV shows the function $X\left(k_{1}, k_{2}\right)$ of the final 2$\mathrm{D}$ array after two phases of the 1-D FFT operations. Both indices $k_{1}$ and $k_{2}$ are in the digit-reverse order. Conversely, if the indices of $x\left(n_{l}, n_{2}\right)$ are in the digit-reverse order, the indices of the output array $X\left(k_{1}, k_{2}\right)$ will be in the linear order.

Table II: Pattern of an 8x8 2-D Linear Array

\begin{tabular}{|llllllll|}
\hline $\mathrm{x}(0,0)$ & $\mathrm{x}(1,0)$ & $\mathrm{x}(2,0)$ & $\mathrm{x}(3,0)$ & $\mathrm{x}(4,0)$ & $\mathrm{x}(5,0)$ & $\mathrm{x}(6,0)$ & $\mathrm{x}(7,0)$ \\
$\mathrm{x}(0,1)$ & $\mathrm{x}(1,1)$ & $\mathrm{x}(2,1)$ & $\mathrm{x}(3,1)$ & $\mathrm{x}(4,1)$ & $\mathrm{x}(5,1)$ & $\mathrm{x}(6,1)$ & $\mathrm{x}(7,1)$ \\
$\mathrm{x}(0,2)$ & $\mathrm{x}(1,2)$ & $\mathrm{x}(2,2)$ & $\mathrm{x}(3,2)$ & $\mathrm{x}(4,2)$ & $\mathrm{x}(5,2)$ & $\mathrm{x}(6,2)$ & $\mathrm{x}(7,2)$ \\
$\mathrm{x}(0,3)$ & $\mathrm{x}(1,3)$ & $\mathrm{x}(2,3)$ & $\mathrm{x}(3,3)$ & $\mathrm{x}(4,3)$ & $\mathrm{x}(5,3)$ & $\mathrm{x}(6,3)$ & $\mathrm{x}(7,3)$ \\
$\mathrm{x}(0,4)$ & $\mathrm{x}(1,4)$ & $\mathrm{x}(2,4)$ & $\mathrm{x}(3,4)$ & $\mathrm{x}(4,4)$ & $\mathrm{x}(5,4)$ & $\mathrm{x}(6,4)$ & $\mathrm{x}(7,4)$ \\
$\mathrm{x}(0,5)$ & $\mathrm{x}(1,5)$ & $\mathrm{x}(2,5)$ & $\mathrm{x}(3,5)$ & $\mathrm{x}(4,5)$ & $\mathrm{x}(5,5)$ & $\mathrm{x}(6,5)$ & $\mathrm{x}(7,5)$ \\
$\mathrm{x}(0,6)$ & $\mathrm{x}(1,6)$ & $\mathrm{x}(2,6)$ & $\mathrm{x}(3,6)$ & $\mathrm{x}(4,6)$ & $\mathrm{x}(5,6)$ & $\mathrm{x}(6,6)$ & $\mathrm{x}(7,6)$ \\
$\mathrm{x}(0,7)$ & $\mathrm{x}(1,7)$ & $\mathrm{x}(2,7)$ & $\mathrm{x}(3,7)$ & $\mathrm{x}(4,7)$ & $\mathrm{x}(5,7)$ & $\mathrm{x}(6,7)$ & $\mathrm{x}(7,7)$ \\
\hline
\end{tabular}

Table III: Pattern of 2-D Array After 1-D FFT for Each Row

\begin{tabular}{|llllllll|}
\hline $\mathrm{F}(0,0)$ & $\mathrm{F}(2,0)$ & $\mathrm{F}(4,0)$ & $\mathrm{F}(6,0)$ & $\mathrm{F}(1,0)$ & $\mathrm{F}(3,0)$ & $\mathrm{F}(5,0)$ & $\mathrm{F}(7,0)$ \\
$\mathrm{F}(0,1)$ & $\mathrm{F}(2,1)$ & $\mathrm{F}(4,1)$ & $\mathrm{F}(6,1)$ & $\mathrm{F}(1,1)$ & $\mathrm{F}(3,1)$ & $\mathrm{F}(5,1)$ & $\mathrm{F}(7,1)$ \\
$\mathrm{F}(0,2)$ & $\mathrm{F}(2,2)$ & $\mathrm{F}(4,2)$ & $\mathrm{F}(6,2)$ & $\mathrm{F}(1,2)$ & $\mathrm{F}(3,2)$ & $\mathrm{F}(5,2)$ & $\mathrm{F}(7,2)$ \\
$\mathrm{F}(0,3)$ & $\mathrm{F}(2,3)$ & $\mathrm{F}(4,3)$ & $\mathrm{F}(6,3)$ & $\mathrm{F}(1,3)$ & $\mathrm{F}(3,3)$ & $\mathrm{F}(5,3)$ & $\mathrm{F}(7,3)$ \\
$\mathrm{F}(0,4)$ & $\mathrm{F}(2,4)$ & $\mathrm{F}(4,4)$ & $\mathrm{F}(6,4)$ & $\mathrm{F}(1,4)$ & $\mathrm{F}(3,4)$ & $\mathrm{F}(5,4)$ & $\mathrm{F}(7,4)$ \\
$\mathrm{F}(0,5)$ & $\mathrm{F}(2,5)$ & $\mathrm{F}(4,5)$ & $\mathrm{F}(6,5)$ & $\mathrm{F}(1,5)$ & $\mathrm{F}(3,5)$ & $\mathrm{F}(5,5)$ & $\mathrm{F}(7,5)$ \\
$\mathrm{F}(0,6)$ & $\mathrm{F}(2,6)$ & $\mathrm{F}(4,6)$ & $\mathrm{F}(6,6)$ & $\mathrm{F}(1,6)$ & $\mathrm{F}(3,6)$ & $\mathrm{F}(5,6)$ & $\mathrm{F}(7,6)$ \\
$\mathrm{F}(0,7)$ & $\mathrm{F}(2,7)$ & $\mathrm{F}(4,7)$ & $\mathrm{F}(6,7)$ & $\mathrm{F}(1,7)$ & $\mathrm{F}(3,7)$ & $\mathrm{F}(5,7)$ & $\mathrm{F}(7,7)$ \\
\hline
\end{tabular}


Table IV: Pattern of 2-D Array After 2-D FFT

\begin{tabular}{|llllllll|}
\hline $\mathrm{X}(0,0)$ & $\mathrm{X}(2,0)$ & $\mathrm{X}(4,0)$ & $\mathrm{X}(6,0)$ & $\mathrm{X}(1,0)$ & $\mathrm{X}(3,0)$ & $\mathrm{X}(5,0)$ & $\mathrm{X}(7,0)$ \\
$\mathrm{X}(0,4)$ & $\mathrm{X}(2,4)$ & $\mathrm{X}(4,4)$ & $\mathrm{X}(6,4)$ & $\mathrm{X}(1,4)$ & $\mathrm{X}(3,4)$ & $\mathrm{X}(5,4)$ & $\mathrm{X}(7,4)$ \\
$\mathrm{X}(0,1)$ & $\mathrm{X}(2,1)$ & $\mathrm{X}(4,1)$ & $\mathrm{X}(6,1)$ & $\mathrm{X}(1,1)$ & $\mathrm{X}(3,1)$ & $\mathrm{X}(5,1)$ & $\mathrm{X}(7,1)$ \\
$\mathrm{X}(0,5)$ & $\mathrm{X}(2,5)$ & $\mathrm{X}(4,5)$ & $\mathrm{X}(6,5)$ & $\mathrm{X}(1,5)$ & $\mathrm{X}(3,5)$ & $\mathrm{X}(5,5)$ & $\mathrm{X}(7,5)$ \\
$\mathrm{X}(0,2)$ & $\mathrm{X}(2,2)$ & $\mathrm{X}(4,2)$ & $\mathrm{X}(6,2)$ & $\mathrm{X}(1,2)$ & $\mathrm{X}(3,2)$ & $\mathrm{X}(5,2)$ & $\mathrm{X}(7,2)$ \\
$\mathrm{X}(0,6)$ & $\mathrm{X}(2,6)$ & $\mathrm{X}(4,6)$ & $\mathrm{X}(6,6)$ & $\mathrm{X}(1,6)$ & $\mathrm{X}(3,6)$ & $\mathrm{X}(5,6)$ & $\mathrm{X}(7,6)$ \\
$\mathrm{X}(0,3)$ & $\mathrm{X}(2,3)$ & $\mathrm{X}(4,3)$ & $\mathrm{X}(6,3)$ & $\mathrm{X}(1,3)$ & $\mathrm{X}(3,3)$ & $\mathrm{X}(5,3)$ & $\mathrm{X}(7,3)$ \\
$\mathrm{X}(0,7)$ & $\mathrm{X}(2,7)$ & $\mathrm{X}(4,7)$ & $\mathrm{X}(6,7)$ & $\mathrm{X}(1,7)$ & $\mathrm{X}(3,7)$ & $\mathrm{X}(5,7)$ & $\mathrm{X}(7,7)$ \\
\hline
\end{tabular}

Comparing Table II and Table IV, the function of the 2-D digit reverse has the following relation:

$$
d r\left(n_{1}, n_{2}\right)=\left(d r\left(n_{1}\right), d r\left(n_{2}\right)\right)=\left(k_{1}, k_{2}\right)
$$

The memory device is generally a 1-D array. In appendix A, a discussion on how to use the 1-D $N_{1}{ }^{*} N_{2}$ point FFT to realize the 2-D FFT for the 2-D array implementation will be presented. $N_{1}$ and $N_{2}$ denote the dimensions of the vectors.

\section{Multi-Dimensional Fast Fourier Transform}

The column-row or row-column approach for the 2-D FFT can be extended to the Multi-Dimensional (M-D) FFT case. The three-dimensional FFT discussed here can be extended to multi-dimensional FFT. A three-dimensional (3-D) Discrete Fourier Transform is represented by

$$
\begin{gathered}
X\left(k_{1}, k_{2}, k_{3}\right)=\sum_{n_{1}=0}^{N_{1}-1} \sum_{n_{2}=0}^{N_{2}-1} \sum_{n_{3}=0}^{N_{3}-1} x\left(n_{1}, n_{2}, n_{3}\right) W_{N_{1}}^{n_{1} k_{1}} W_{N_{2}}^{n_{2} k_{2}} W_{N_{3}}^{n_{3} k_{3}} \\
\text { for } n=0 \leq k_{i} \leq N_{i}-1
\end{gathered}
$$


It can be reordered into the 1-D DFT expression as follows

$$
X\left(k_{1}, k_{2}, k_{3}\right)=\sum_{n_{3}=0}^{N_{3}-1}\left\{\sum_{n_{2}=0}^{N_{2}-1}\left[\sum_{n_{1}=0}^{N_{1}-1} x\left(n_{1}, n_{2}, n_{3}\right) W_{N_{1}}^{n_{1} k_{1}}\right] W_{N_{2}}^{n_{2} k_{2}}\right\} W_{N_{3}}^{n_{3} k_{3}}
$$

Thus, the 3-D FFT can be calculated by employing the following steps:

(1) 1-D $N_{1}$-point FFT $N_{2}{ }^{*} N_{3}$ times first,

(2) 1-D $N_{2}$-point FFT $N_{I} * N_{3}$ next, and

(3) finally, 1-D $N_{3}$-point FFT $N_{1} * N_{2}$ times.

After the 3-D FFT calculation, the indices $n_{i}$ and $k_{i}$ will be in digit-reverse order in each vector as follows:

$$
d r\left(n_{1}, n_{2}, n_{3}\right)=\left(d r\left(n_{1}\right), d r\left(n_{2}\right), d r\left(n_{3}\right)\right)=\left(k_{1}, k_{2}, k_{3}\right)
$$

The basic execution modules are unchanged for 1-D to M-D FFT if the column-row or row-column approach is implemented.

\section{Discrete Cosine Transform by FFT}

The Discrete Cosine Transform (DCT) is a fundamental image processing algorithm for many image and video compression systems because of its fast computation and easy implementation. This section will discuss three methods of employing the chip set to implement the DCT. The DCT is defined as follows:

$$
X(0)=\left(\frac{1}{N}\right)^{1 / 2} \sum_{n=0}^{N-1} x(n)
$$




$$
(k)=\left(\frac{2}{N}\right)^{1 / 2} \sum_{n=0}^{N-1} x(n) \cos \left[\frac{\pi(2 n+1) k}{2 N}\right] \quad \text { for } k=1,2, \ldots, N-
$$

and the Inverse Discrete Cosine Transform (IDCT) is defined as follows:

$$
\begin{array}{r}
x(n)=\left(\frac{1}{N}\right)^{1 / 2} X(0)+\left(\frac{2}{N}\right)^{1 / 2} \sum_{k=1}^{N-1} X(k) \cos \left[\frac{\pi(2 n+1) k}{2 N}\right] \\
\text { for } n=0,1, \ldots, N-1
\end{array}
$$

\section{Method 1: N-Point DCT by Zero Extended 2N-Point FFT}

The first method of computing N-point DCT extends the sequence from $\mathrm{N}$ points to $2 \mathrm{~N}$ points by padding zeros to the last $\mathrm{N}$ points of data. The DCT from Equations (2.30) and (2.31) can be expressed as

$$
X(k)=\operatorname{Re}\left[c(k) e^{-j \pi k /(2 N)} \sum_{n=0}^{2 N-1} x(n) W_{2 N}^{k n}\right] \quad \text { for } \quad k=0,1, \ldots, N-1
$$

where

$$
c(k)=\left\{\begin{array}{l}
\left(\frac{1}{N}\right)^{1 / 2} \quad k=0 \\
\left(\frac{2}{N}\right)^{1 / 2} \text { otherwise }
\end{array}\right.
$$

Equation (2.33) means that the N-point DCT can be obtained by executing the $2 \mathrm{~N}$ point real FFT first and then taking the real part of complex multiplications as shown in Figure 8. Thus, the DCT can be implemented by the LH9124 in three phases. The first computes the N-point FFT. The second computes the recombining process. The final 
phase computes complex vector multiplications.

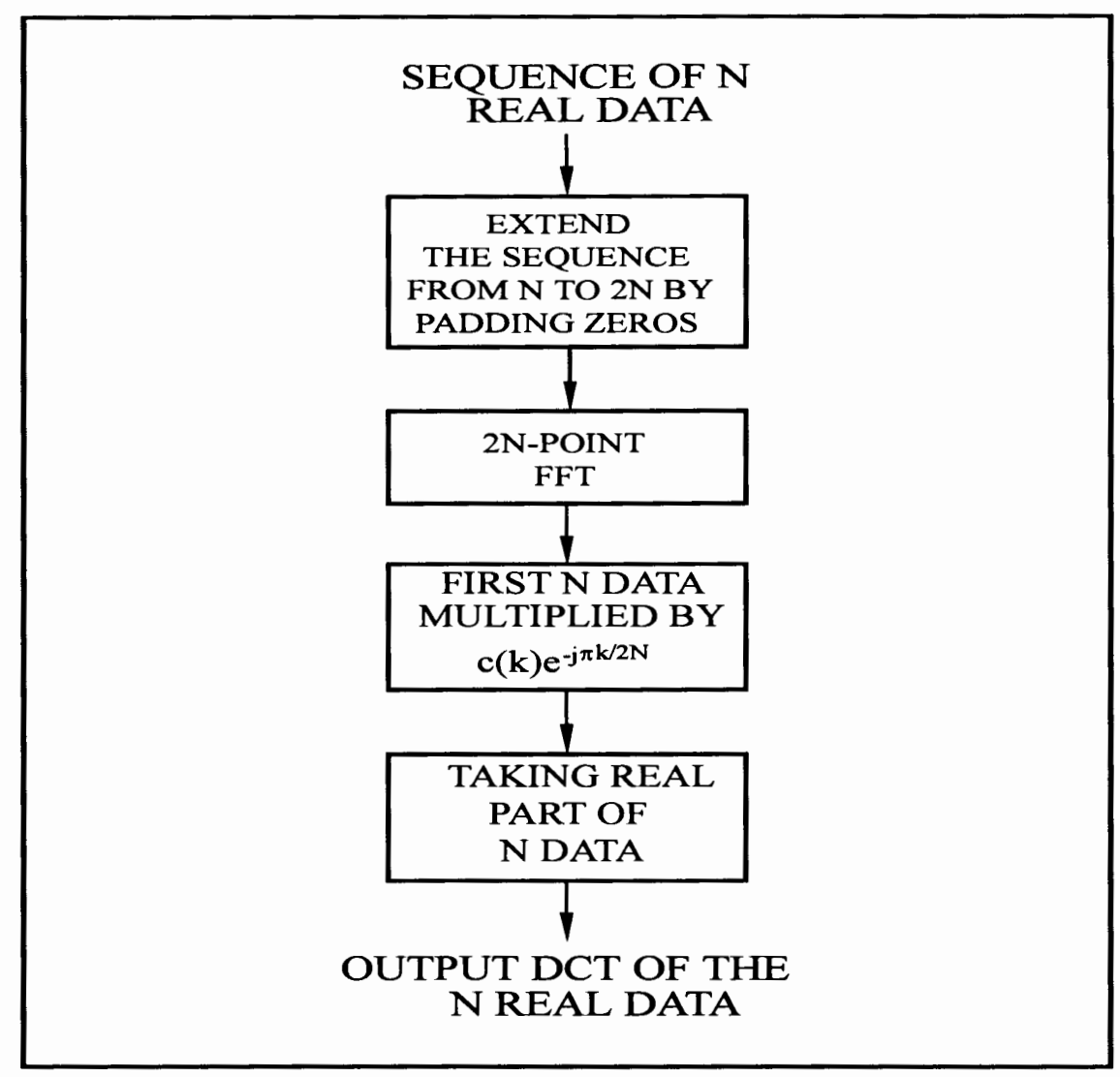

Figure 8. N-Point DCT by Zero Extended 2N-Point FFT

\section{Method 2: N-point DCT by Symmetric Extended 2N-Point FFT}

The second method of computing N-point DCT also extends the sequence from $\mathrm{N}$ points to $2 \mathrm{~N}$ points. However, the extended $2 \mathrm{~N}$-point sequence is symmetric and defined as follows:

$$
\hat{x}(n)= \begin{cases}x(n) & n=0,1, \ldots, N-1 \\ x(2 N-1-n) & n=N, N+1, \ldots, 2 N-1\end{cases}
$$

Then, the DFT of $\hat{x}(n)$ is given by 


$$
\hat{X}(k)=\sum_{n=0}^{2 N-1} \hat{x}(n) W_{2 N}^{n k}=2 e^{j \pi \frac{k}{2 N}} \sum_{n=0}^{N-1} x(n) \cos \frac{\pi(2 n+1) k}{2 N}
$$

Comparing Equation (2.36) with Equation (2.31), the DCT can be expressed as

$$
X(k)=\frac{c(k)}{2} e^{-j \pi k / 2 N} \hat{X}(k) \quad \text { for } \quad k=0,1, \ldots, N-1
$$

Thus, the N-point DCT can be obtained by executing the $2 \mathrm{~N}$-point real FFT first and then adjusting the results by complex multiplications as shown in Figure 9. As in the first method, the DCT can also be implemented in the same three phases.

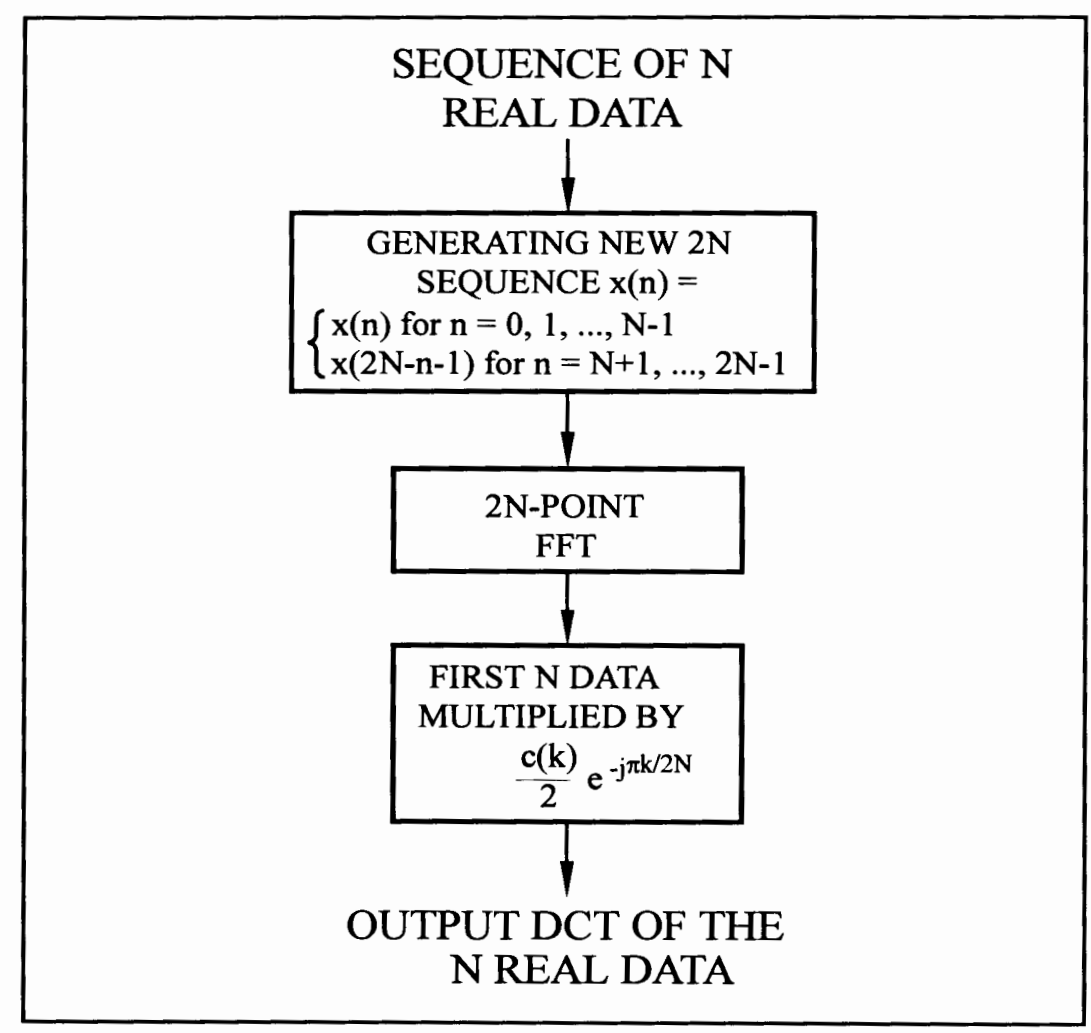

Eigure 9. N-Point DCT by Symmetric Extended 2N-Point FFT

\section{Method 3: N-Point DCT by N-Point FFT}

The first two methods introduce inefficiency in computation by extending the data 
sequence. In method 1 , the sequence is extended to the length of $2 \mathrm{~N}$ by zero padding. In method 2 , the sequence is extended to a $2 \mathrm{~N}$ symmetry. Method 3 directly computes the rearranged $\mathrm{N}$-sequence data instead of extending the data sequence. The last method is obviously better than the first two because Sharp chip set can compute two sequences of data with this method at the same time and improve the speed performance. Here, the new data sequence $y(n)$ derived from the original sequence $x(n)$ is represented by

$$
y(n)= \begin{cases}x(2 n) & n=0,1, \ldots, N / 2-1 \\ x(2 N-2 n-1) & n=N / 2, N / 2+1, \ldots, N-1\end{cases}
$$

Thus, the N-point DCT is obtained from the N-point FFT instead of the 2N-point FFT.

$$
\begin{aligned}
& \hat{X}(k)=\sum_{n=0}^{N-1} x(n) \cos \frac{(2 n+1) k \pi}{2 N} \\
& =\sum_{n=0}^{N / 2-1} x(2 n) \cos \frac{(4 n+1) k \pi}{2 N}+\sum_{n=0}^{N / 2-1} x(2 n+1) \cos \frac{(4 n+3) k \pi}{2 N}
\end{aligned}
$$

Setting $n=N-n-1$ in the second term of Equation (2.39), we may get

$$
\begin{aligned}
& X \hat{(k)}=\sum_{n=0}^{N / 2-1} y(n) \cos \frac{(4 n+1) k \pi}{2 N}+\sum_{n=N / 2}^{N-1} y(n) \cos \frac{(4 n+1) k \pi}{2 N} \\
& =\sum_{n=0}^{N-1} y(n) \cos \frac{(4 n+1) k \pi}{2 N}
\end{aligned}
$$

Thus, the DCT can be computed from the following equation:

$$
X(k)=\operatorname{Re}\left[c(k) e^{-j \pi(k / 2 N)} \sum_{n=0}^{N-1} y(n) W_{N}^{n k}\right]
$$

It can also be derived that 


$$
\begin{array}{r}
X(N-k)=\operatorname{Im}\left[c(k) e^{-j \pi(k /(2 N))} \sum_{n=0}^{N-1} y(n) W_{N}^{n k}\right] \\
\text { for } \quad k=0,1, \ldots, N^{\prime 2}
\end{array}
$$

This means that only the first $(\mathrm{N} / 2+1)$ points of the complex sequence can be computed. The first half of the DCT sequence can be obtained from the real part of the complex sequence and the second half of the DCT sequence can be obtained from the imaginary part of the complex sequence. The N-point DCT can be obtained by computing $\mathrm{N}$-point real FFT first and then adjusting the results by complex multiplications as shown in Figure 10.

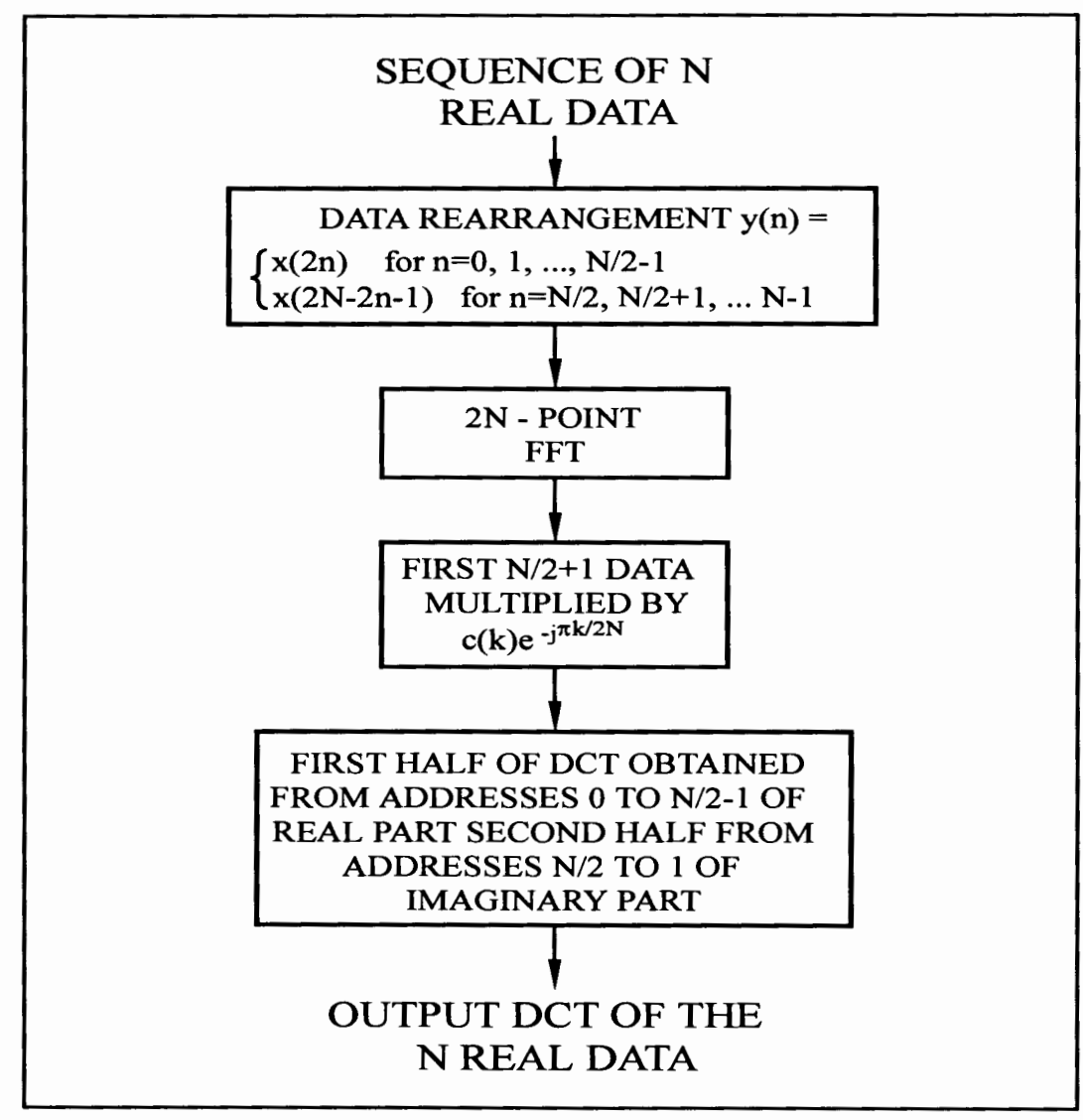

Eigure 10. N-Point DCT of the N Real Data 


\section{CHAPTER III}

\section{BRIEF DESCRIPTIONS OF SHARP LH9124 AND LH9320}

Array digital signal processing has become increasingly important because of the advancement of DSP algorithms and VLSI technologies [Haykin, 1985]. DSP deals with processing of propagating signals received by an array of sensors. The computing speed of an array processor usually constrains system performance. Therefore, sampling rate and number of the array sensors must be confined or the system modeling adaptability must be simplified. To overcome the speed bottleneck of array processing and to obtain the flexibility in system design, an array chip set (LH9124 and LH9320) has been developed by Sharp Microelectronics Technology.

The LH9124 is a high-performance block floating-point digital signal processor. The computation process is a procedure in which the original array is normalized to the far left of the computer word, with the restriction that $|x[n]|<1$. This is a fixed-point procedure, except that after every addition there is an overflow test. The LH9124 unit consists of four I/O ports for flexible data flow control. The instruction set is defined in a function-level representation and consists of five instruction categories: Digital Signal Processing (DSP), Complex Arithmetic Instructions (CAI), Vector Arithmetic Instructions (VAI), Vector Logic Instructions (VLI) and General Purpose Instructions (GPI) [LH9124 User's Guide]. FFT instructions provided by the LH9124 are listed in

Table V. These instructions can be found under DSP categories. DSP functions are 
optimized for highly parallel and pipelined forms which built into the data path. Therefore, the Sharp LH9124 can obtain up to 680 million operations per second. For detailed information about the instructions, see the Sharp LH9124 User Guide manual.

Table V: The LH9124 Instruction Set

\begin{tabular}{|c|c|}
\hline FUNCTIONS & DESCRIPTION \\
\hline \multicolumn{2}{|r|}{ DSP INSTRUCTIONS } \\
\hline BFLY2 & Performs Radix-2 butterfly operation. \\
\hline BFLY4 & Performs Radix-4 butterfly operation. \\
\hline BFLY16 & Performs Radix-16 operation. \\
\hline BWND2 & Performs Radix-2 with complex window function. \\
\hline BWND4 & Performs Radix-4 with complex window function. \\
\hline BFCT & Performs Fast cosine transform/double length operation. \\
\hline $\mathrm{B}[\mathrm{C} / \mathrm{D} / \mathrm{R}] \mathrm{FIR}$ & $\begin{array}{l}\text { Performs Complex or Double or Real finite impulse } \\
\text { response filter operation. }\end{array}$ \\
\hline \multicolumn{2}{|r|}{ COMPLEX ARITHMETIC INSTRUCTIONS } \\
\hline CADD & Performs complex addition operation. \\
\hline CMAG & Performs complex magnitude squared operation. \\
\hline CMUL & Performs complex multiplication operation. \\
\hline CSUB & Performs complex substraction operation. \\
\hline \multicolumn{2}{|r|}{ VECTOR ARITHMETIC INSRUCTIONS } \\
\hline VABS & Performs vector absolute operation. \\
\hline VADD/VSUB & Performs vector addition or substraction operation. \\
\hline VMUL & Performs vector multiplication operation. \\
\hline VMXM & Performs vector maximum and minimum operation. \\
\hline \multicolumn{2}{|r|}{ VECTOR LOGICAL INSTRUCTIONS } \\
\hline VNAND & Performs vector logical NAND operation. \\
\hline VNOR & Performs vector logical NOR operation. \\
\hline VPAS & Performs vector logical PASS operation. \\
\hline VXNOR & Performs vector logical NOR operation. \\
\hline \multicolumn{2}{|r|}{ GENERAL PURPOSE INSTRUCTIONS } \\
\hline MOVC & $\begin{array}{l}\text { Move coefficient data from } C \text { port to another }(A, B, Q) \\
\text { port. }\end{array}$ \\
\hline MOVD & Move data from $(A, B, Q)$ to another port $(A, B, C, Q)$ port. \\
\hline
\end{tabular}


Table VI: The LH9320 Address Generator Instruction Set for FFT

\begin{tabular}{|l|l|}
\hline INSTRUCTION & \multicolumn{1}{c|}{ DESCRIPTION } \\
\hline \hline BF2 $\mathrm{i}$ & Data address sequence for $\mathrm{i}$-th column radix-2 \\
\hline BF4i & Data address sequence for $(2 * \mathrm{i})$-th column radix-4 \\
\hline MXB24i & Data address sequence for $(2 * \mathrm{i}+1)$-th column radix-4 \\
\hline BF16i & Data address sequence for $(4 * \mathrm{i})$-th column radix-16 \\
\hline MXB216i & Data address sequence for $(4 * \mathrm{i}+1)$-th column radix-16 \\
\hline MXB416i & Data address sequence for $(4 * \mathrm{i}+2)$-th column radix-16 \\
\hline MXB2416i & Data address sequence for $(4 * \mathrm{i}+3)$-th column radix-16 \\
\hline TF2i & Twiddle factor address sequence for $\mathrm{i}$-th column radix-2 \\
\hline TF4i & Twiddle factor address sequence for $\mathrm{i}$-th $(2 * \mathrm{i})$-th radix-4 \\
\hline MXT24i & Twiddle factor address sequence for $(2 * \mathrm{i}+1)$-th column radix-4 \\
\hline TF16i & Twiddle factor address sequence for $(4 * \mathrm{i})$-th column radix-16 \\
\hline MXT216i & Twiddle factor address sequence for $(4 * \mathrm{i}+1)$-th column radix-16 \\
\hline MXT416i & Twiddle factor address sequence for $(4 * \mathrm{i}+2)$-th column radix-16 \\
\hline MXT2416i & Twiddle factor address sequence for $(4 * \mathrm{i}+3)$-th column radix-16 \\
\hline RBF0 & Linear Sequence to Digit-reverse sequence conversion \\
\hline
\end{tabular}

The LH9320 is a programmable address generator that provides the address patterns required by the LH9124. It can generate one 24-bit address per cycle for input, output or coefficient sequence to the LH9124 DSP chip. It posseses not only the general features such as linear, modulo, and bit-reverse addressing contained by the general purpose DSP chips but also special features for the LH9124 to optimize the performance of some DSP functions [LH9320 User's Guide]. The FFT addressing instructions for the LH9320 are listed in Table VI. If the $\mathrm{j}$-th column is implemented by a radix- 2 stage, the instructions $\mathrm{BF} 2 \mathrm{j}$ and $\mathrm{TF} 2 \mathrm{j}$ are used to generate the required data and address patterns. If the $\mathrm{j}$-th column is the beginning column of a radix-4 stage, the instructions BF4 $\mathrm{i}$ and TF4 $\mathrm{i}$ will be implemented when $\mathrm{j}=2 * \mathrm{i}$ or the instructions MXB24i and MXT24i will be implemented when $j=2 * i+1$. Similarly, we can select instructions for a radix-16 stage. Based on the novel algorithms of defining 1-D indexing for M-D mixed radix FFT 
implementations [Ju, 1993], some instructions of the LH9320 can be universally employed to the array with an arbitrary numbers of dimensions.

\section{Design Considerations of LH9124}

The design goal of the chip set is that the strict real-time requirement of short computing latency or fast data throughput of array digital signal processing can be obtained at a reasonable cost. The design considerations for chip users to achieve this goal are discussed below based on the following five categories: (1) algorithm, (2) arithmetic, (3) data path structure, (4) instructions, and (5) system configuration.

\section{Algorithm:}

Block implementation is adopted since it allows for efficient use of parallel processors and fast block algorithms such as Fast Fourier Transform (FFT) to achieve speed gains. The term "block" refers to radix- $n$ (where $n$ can be either 2,4 or 16) FFT decomposed from an N-point DFT with complex arithmetic operations (see Figure 3). The chip set emphasizes implementing block oriented DSP algorithms to solve problems through frequency-domain. In addition, array processing usually has to handle multidimensional data. The performance of the FFT algorithms regardless of the dimension is then the main concern of the design.

The Multi-Dimension (M-D) indexing for the M-D FFT is usually the performance killer of M-D array processing. In order to solve it, a novel FFT SFG implementation has been developed by C. J. Ju [Ju, 1993] to compute the M-D FFT as the 1-D FFT. There are many advantages to this approach. First, the unified 1-D addressing 
for the 1-D to M-D FFT can be constructed. This unified addressing simplifies the design of the LH9320 to support M-D problems. In addition, the optimized instruction can be directly extracted from the vector-matrix representation of the algorithm. Second, the structure optimization for the FFT is reduced to the 1-D problem. Third, the concept of M-D to 1-D FFT mapping can be extended to solve other block oriented DSP algorithms, such as FIR and IIR [Ju and Alexander, 1987].

\section{Arithmetic:}

The block floating-point arithmetic (see "Block Floating-point Arithmetic and Implementation" on page 46) has been adopted by the LH9124 for the following reasons. First, block implementation is adopted and the FFT structure is inherently suitable for the block floating-point arithmetic. Second, it has the advantages of both floating-point and integer arithmetic. One exponent register can be used for all the block data to extend the dynamic range of the signal as done by the floating-point arithmetic. Thus, addition and multiplication operations can be implemented as easily as the corresponding fixed-point arithmetic operations. Third, the $\mathrm{A} / \mathrm{D}$ and $\mathrm{D} / \mathrm{A}$ devices have integer format. Therefore, both time and cost of performing the data conversion can be saved. It should be noted that there is one drawback of block arithmetic, i.e. the scaling for the block floating-point arithmetic.

\section{Data Path Structure:}

The LH9124 is a pass-based processor where each opcode and related data flow opcode is valid for one pass. By pass-based, we mean that for a single instruction 
operation, the data from the input port is passed directly to the transform engine and to the output port without going through the holding registers and execution unit (see Figure 11). Data must be arranged according to the address sequences required before it is passed to the LH9124. The basic computing modules such as radix-2, radix-4, and radix-16 butterflies are built in the data path with the optimized parallel and pipelined structures. There are two reasons for the pass-based form design. First, a cascaded or parallel architecture can be built to meet the strict latency and throughput requirements. Second, instructions can be efficiently performed regardless of number of data and the array dimension.

\section{Instructions:}

The instructions from the instruction set of LH9124 are compact and are carried out at a macro level. These instructions are hardwired in the chip architecture. No complex programming for the instructions is required. The instructions are defined in a function-level representation. By function-level representation, we mean that the instructions are a set of complex arithmetic operations. For example, the instruction BFLY2 consists of a set of complex multiplications and additions to perform the radix-2 operation. Generally, the DSP application can be partitioned into several function block diagrams. The function-level representation of instructions can be directly mapped from the function block diagram. In another word, one can assume the function block diagram is function-level representation in graphical environment. Thus, no time-consuming software coding and debugging process are necessary. Users consider applications only at the function block diagram level. A window-based function simulator is provided to help 
users develop DSP applications.

Another reason for the function block instruction is that the efficiency of parallel processing comes mainly from partitioning data and function. The function is already built in the data path with an optimized parallel and pipelined structure. Users need only to consider the data partition in constructing parallel systems. When the data is wellpartitioned, speed improvements are usually proportional to the number of execution units employed.

\section{System:}

The address generating instruction set of the LH9320 is well-defined for the FFT to support both unit-processor and multi-processor systems. Moreover, the LH9124 includes 4-bidirectional 48-pin I/O ports. The four I/O port design provides users flexibility in system configuration. Users can use this flexibility either to optimize the system performance or to reduce the system cost. In addition, a parallel or cascaded system architecture can be developed with little or no extra hardware. 


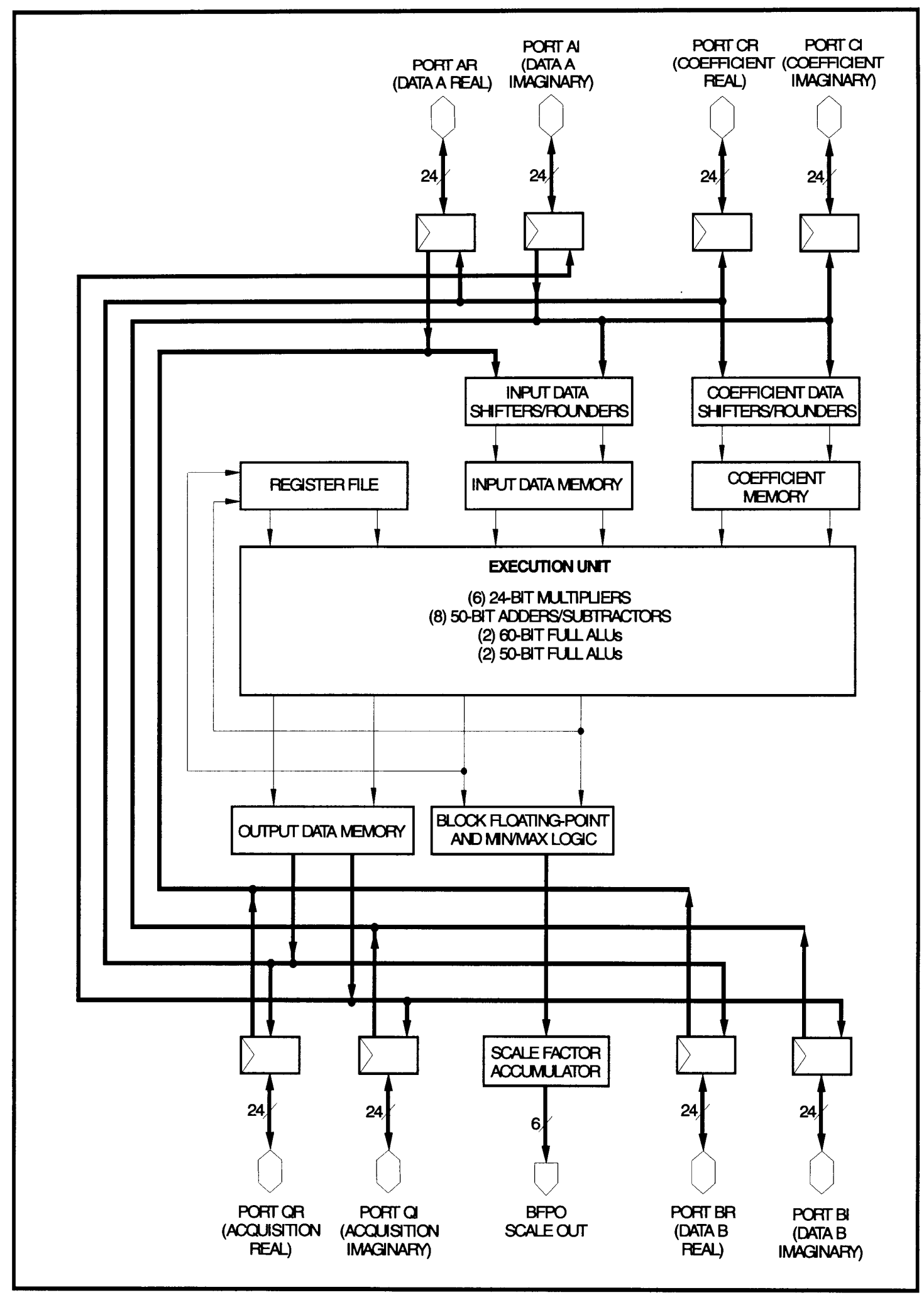

Eigure 11. LH9124 Detailed Block Diagram and Execution Unit. 


\section{Unified Signal Flow Graph Structure for 1-D to M-D FFT}

The architecture of LH9124 suitably and conveniently matches the concept of the unified addressing for the 1-D to M-D FFT algorithm. This section will show that a unified Signal Flow Graph (SFG) structure representing the 1-D to M-D FFT can be constructed. Conceptually, the M-D FFT algorithm can be easily understood and realized. Practically, the unified SFG structure implies the unified 1-D indexing, of data, coefficient factor, and bit-reverse for 1-D to M-D FFT implementation and simplifies the M-D FFT addressing.

A novel vector-matrix representation of FFT is proposed in Chapter IV. From the Discrete Fourier Transform (DFT) to FFT decomposition, each stage of the FFT can be described by three matrices: the right permutation matrix indicating the input interconnection, the center matrix indicating the butterfly operation, and the left permutation matrix indicating the output interconnection. It can be derived from the results obtained in Chapter IV that all the FFTs with the same number of elements regardless of dimensions have the same permutation matrices. This means that they share the same SFG structure. In addition, the butterfly operation matrices for the K-th vector of 1-D to M-D FFTs are the same. These results imply that the indexing for the 1-D FFT can be employed for that of the M-D FFT.

By the well-known DFT to Decimation-In-Time (DIT) FFT decomposition [Ju, 1993], the vector-matrix form of 1-D FFT with length $N=2^{s}$ can be derived as

$$
X=F G_{s}(B I(s)) \times F G_{s-1}(B I(s-1)) \times \ldots \times F G_{I}(B I(1)) \times \underline{x_{b}}
$$

where $\underline{X}$ and $x_{b}$, respectively, denote the $\mathrm{N}$-point linear output vector and $\mathrm{N}$-point bit 
reverse input vector. $F G_{k}(B S(j))$ represents the k-th stage of the FFT and can be expressed as

$$
F G_{k}(B S(j))=P_{l k} \times B S(j) \times P_{r k}
$$

$B S(j)$ is the butterfly operation matrix with radix-2 modules along the diagonal as

$$
B S(j)=\left[\begin{array}{cccc}
B_{j}(0) & 0 & \ldots & 0 \\
0 & B_{j}(1) & 0 & \ldots \\
\ldots & 0 & \ldots & \ldots \\
\ldots & \ldots & \ldots & \ldots \\
0 & \ldots & \ldots & B_{j}(N / 2-1)
\end{array}\right]
$$

where " 0 " is a 2 by 2 zero matrix and the radix-2 butterfly (see "Fundamental Computing Structures: Butterflies" on page 18$)$ module $B_{j}(n)$ can be represented by

$$
B_{j}(n)=B F_{N}^{i}=\left[\begin{array}{cc}
W_{N}^{0} & W_{N}^{i} \\
W_{N}^{0} & W_{N}^{i+N / 2}
\end{array}\right]
$$

with

$$
i=\frac{N}{2^{j}} \times \operatorname{Int}\left(\frac{n}{2^{s-1}}\right)
$$

$\operatorname{Int}(x)$ denotes the integer part of $x . \quad P_{r k}$ specifies the interconnection between inputs and butterfly modules and is a permutation matrix with its element defined as

$$
P_{r k}(n, m)= \begin{cases}1 & \text { for } m=f_{r k}(n) \\ 0 & \text { otherwise }\end{cases}
$$

where

$$
f_{r k}(n)=\frac{N}{2^{k-1}} \times \operatorname{Mod}(n)_{2^{k-1}}+\operatorname{Int}\left(\frac{n}{2^{k-1}}\right)
$$


$\operatorname{Mod}(x)_{y}$ denotes the modulo operation on the number $\mathrm{x}$ with modulo length $\mathrm{y}$. Since the in-place algorithm is employed, the left permutation matrix $P_{l k}$ is the transpose of the right permutation matrix.

The parallel vector-matrix form for the traditional 2-D FFT implementation with the row length $N_{1}=2^{s_{1}}$, the column length $N_{2}=2^{s_{2}}$, and the total number of the 2-D array elements $N=N_{1} \times N_{2}$ can be derived and represented by the following form

$$
\begin{aligned}
X_{c} & =F G_{s_{2}}\left(B S\left(s_{2}\right)\right) \times \ldots \times F G_{1}(B S(1)) \times P_{N} \\
& \times F G_{s_{1}}(B S(1)) \times \ldots \times F G_{1}(B S(1)) \times \underline{x} b r
\end{aligned}
$$

Where $X_{c}$ is the N-point vector in the column-major order of the linear output array and $\underline{x}_{b r}^{T}$ is the N-point vector in the row-major order of the bit-reversed input array. $P_{N}$ is a transpose matrix to the transform data from the row-major order to column-major order. Combining the permutation matrices of Equation (3.6) with the transpose matrix $P_{N}$ from Equation (3.8), a new form of the 2-D FFT structure is created:

$$
\begin{aligned}
\underline{X}_{r}^{T}= & F G_{s_{1}+s_{2}}\left(B S\left(s_{2}\right)\right) \times \ldots \times F G_{s_{1}+1}(B S(1)) \\
& \times F G_{s_{1}}\left(B S\left(s_{1}\right)\right) \times \ldots \times F G_{1}(B S(1)) \times \underline{x}_{b r}^{T}
\end{aligned}
$$

where $\underline{X}_{r}^{T}$ is an N-point vector in the row-major order of the linear output array. A detailed derivation of Equation (3.1) through Equation (3.9) will be discussed further in chapter IV.

Comparing Equation (3.9) with Equation (3.1) and setting $s=s_{1}+s_{2}$, it can be seen that the $\mathrm{N}_{1}$ by $\mathrm{N}_{2}$ 2-D FFT has the same interconnection structure as the N-point 1-D FFT. It implies that both the equations can have the same SFG structure. Moreover, the butterfly matrices are the same for the k-th stage of the row FFT, column FFT, and 1-D 
FFT.

Figure 12 shows the SFG structure for the 4 by 4 2-D FFT and 16-point 1-D FFT. The upper (or the top) value shows the inputs, outputs, and twiddle factors for the 2-D case and the lower (or the bottom) value is for the 1-D case.

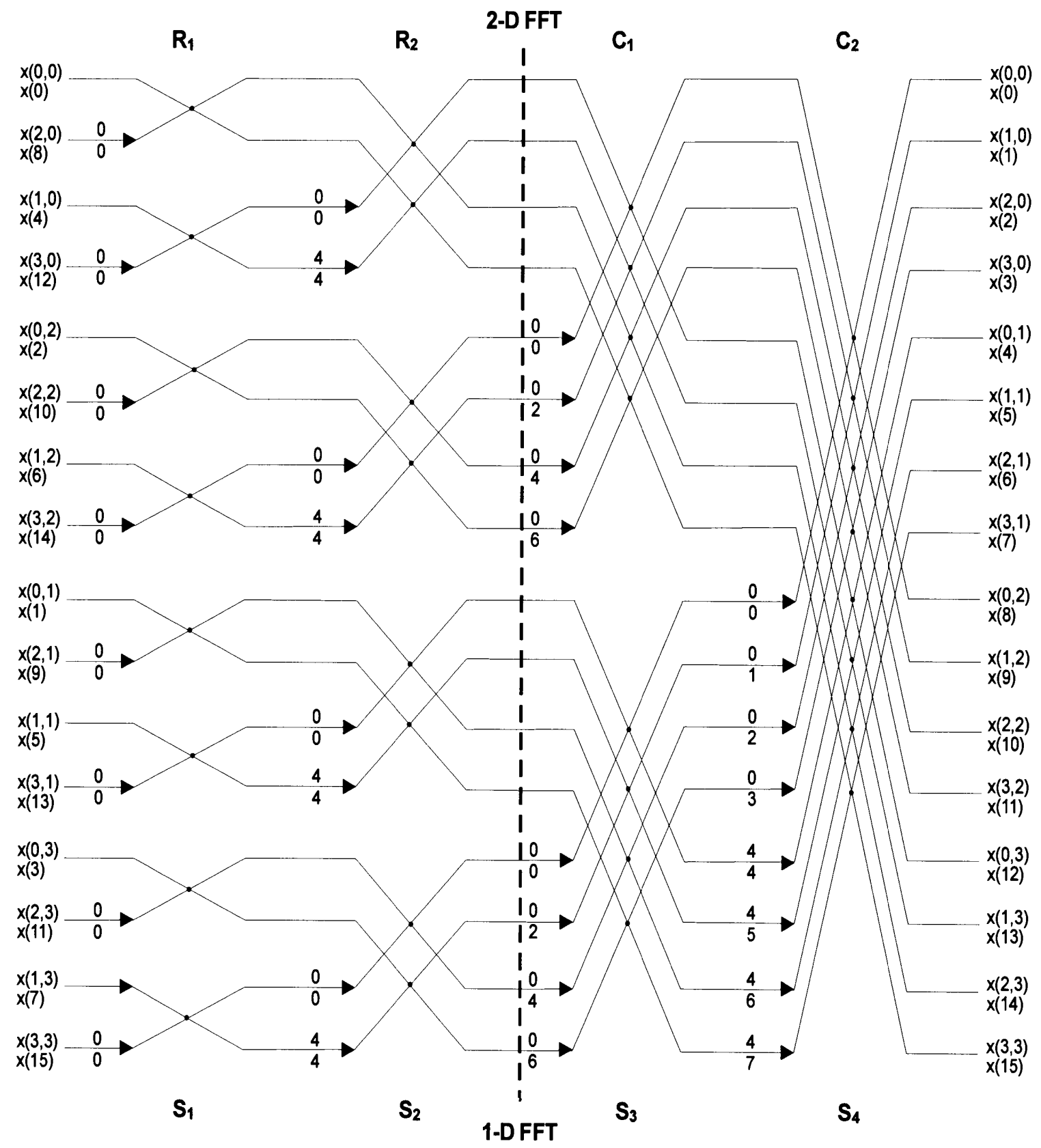

Eigure 12. Signal Flow Graph for 4 by 4 2-D FFT and 16-point 1-D FFT 


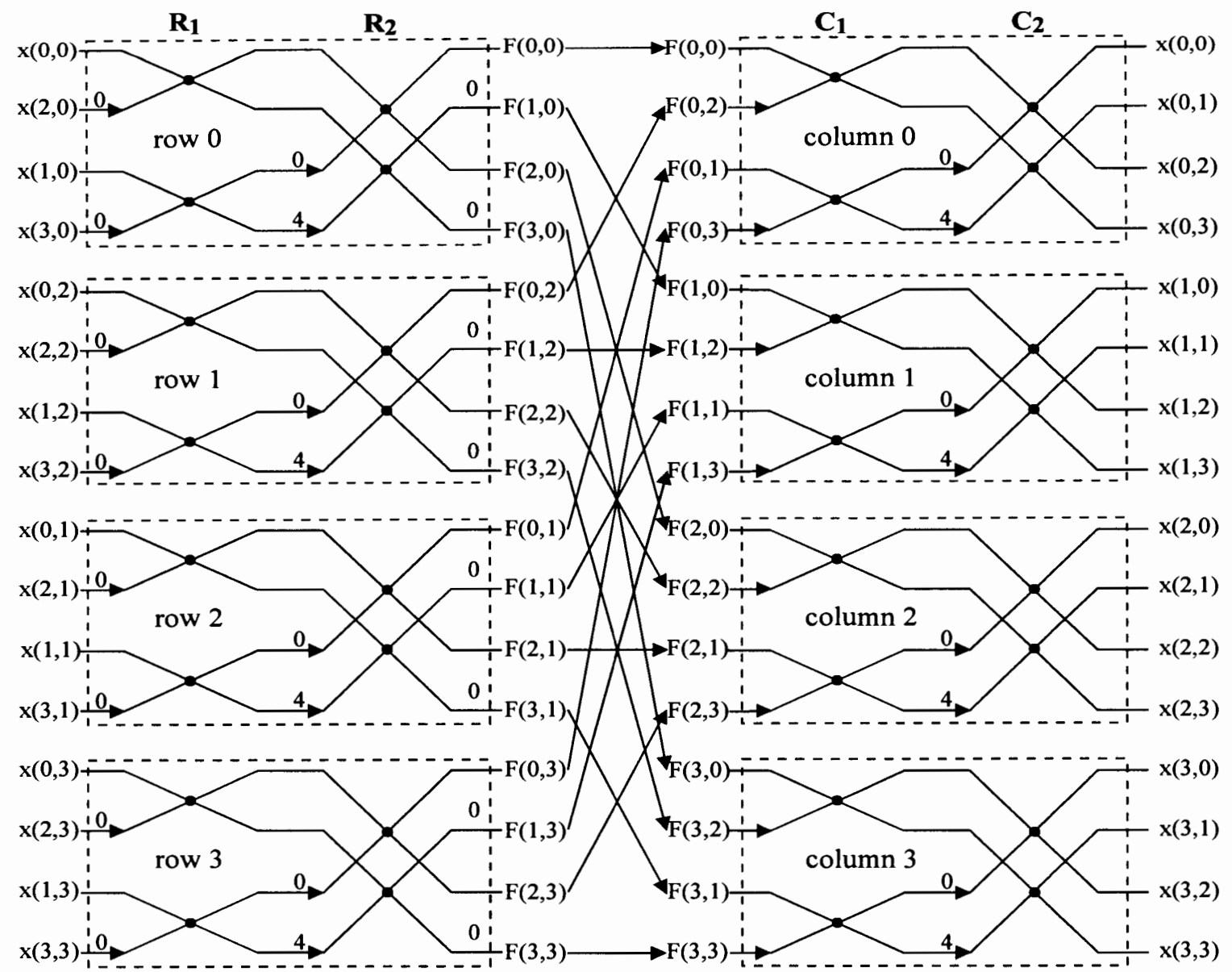

Eigure 13. Signal Flow Graph for Traditional Implementation of 4 by 4 2-D FFT

\section{Block Floating-point Arithmetic and Implementation}

The LH9124 implements block floating-point arithmetic to improve the precision of computations for a fixed-point processor. The FFT algorithm for its inherent block processing structure is suited to block floating-point arithmetic. The block processing structure means that the FFT algorithm is decomposed into a pipeline of computing stages and that the block output data of the current stage is the block input data of the next stage as shown in Figure 3 in page 16. The processor with block floating-point arithmetic can 
have the advantages of both fixed-point and floating-point. First, the architecture is simple because both multiplications and additions are fixed-point. Second, the block floating-point can obtain even higher precision than the floating-point with the same word length. However, these advantages will be offset by the overhead in computations if the scaling problem in block floating-point arithmetic is not well handled. Therefore, it is important to know how the M-D FFT is implemented to avoid the scaling problem of block floating-point arithmetic and it will be discussed below.

From Equation (3.8), the 4 by 4 2-D FFT implementation by the traditional approach is expressed as

$$
\begin{aligned}
\underline{X}_{c}= & F G_{2}\left(B I\left(s_{2}\right)\right) \times \ldots \times F G_{I}(B I(I)) \times P_{N} \\
& \times F G_{2}(B I(2)) \times \ldots \times F G_{I}(B I(l)) \times \underline{x}_{r}^{T}
\end{aligned}
$$

and its SFG structure is shown in Figure 13. The traditional 2-D FFT implementation computes the 1-D FFT for each row, transfers the data from the row-major into the column-major order, and then computes 1-D FFT for each column. The order of computations is from one rectangular block (represented by dotted box) to the other rectangular block shown in Figure 13. After the row FFTs, the number of scaling for each row vector may be different. The difference in scaling is due to the different bit growth in each row. Therefore, the overhead has occurred because the column FFTs cannot be directly computed from these data.

The overhead can be solved by either software or hardware. However, the computing time will be increased by the software approach and the hardware complexity will be increased by the hardware approach. For example, a vector processor solves this 
problem by employing extra hardware to keep tracking the scale factor for each block and the maximum scale factor. The solution by the LH9124/LH9320 is to implement the novel approach of implementing the M-D FFT algorithm. The new approach can eliminate the scaling overhead by considering the whole array as a data block as shown in Figure 12. Only the maximum scale factor has to be kept for each stage.

Fundamentally, the block floating-point mechanism of the LH9124 consists of two parts, the input and the output. The output part is to calculate the maximum magnitude of the output data for the current stage of radix operations. Then, a scaling factor is derived as a reference value for the input scaling of the next stage. The input part receives the scaling factor generated by the previous stage. The number of bits to be shifted right for the current input data will be based on the scaling factor and the instruction to be executed. Therefore, the data overflow and the precision of integer operations are automatically controlled by the block floating-point mechanism. 


\section{CHAPTER IV}

\section{UNIFIED INDEXING OF FFT ALGORITHMS}

It is well-known that the computing efficiency of the FFT comes from the recursive factorization of the twiddle factor matrix of the discrete Fourier transform. To derive the unified addressing for the one dimensional (1-D) to multidimensional (M-D) FFT algorithm, a novel matrix form of factorizing and representing twiddle factor matrix will be presented. Then, it will be also presented that all the matrices have their physical meaning in the practical implementation. Each stage of the FFT is represented by three cascaded matrices. The right permutation matrix specifies the input interconnections and defines the input data sequence. The left permutation matrix specifies the output interconnections and defines the output data sequence. The middle diagonal block matrix performs the butterfly operation and defines the twiddle factor sequence.

Equivalent relationships of these matrices will be introduced in this chapter. It will be shown that one kind of the FFT algorithms can be derived from the other kind of the FFT algorithm through the equivalent transform of the matrices. For example, the inplace bit-reverse inputs and linear outputs (BI/LO) FFT can be derived from the in-place linear inputs and bit-reverse outputs (LI/BO) FFT and vice versa. For definiteness, this chapter discusses the decimation-in-time (DIT) FFT only. Essentially, all the results can be extended to the decimation-in-frequency (DIF) FFT in a straightforward manner.

From the novel vector-matrix representation, an equivalent relationship between 
1-D and M-D FFTs by employing the equivalent transformation of the matrices can be also derived. Therefore, it can be obtained that the signal flow graph (SFG) structure of the 1-D FFT can be used to represent that of the M-D FFT regardless of the dimension, if the total number of elements is the same. The radix-2 FFT is used as an illustration in the discussion, although the implemented approach can be extended to an arbitrary mixed radix FFT.

The unified indexing for 1-D to M-D FFT algorithm has been implemented in the array processor chip set, namely the LH9124/LH9320 developed by Sharp Microelectronics Technology. It can be seen from the chip set implementation that the computing time of the FFT is independent on the dimension of the FFT. Thus, both the 256 by 256 point 2-D complex FFT and the 64K 1-D complex FFT have the same computation time.

\section{1-D Bit-reverse Inputs And Linear Outputs FFT}

The DFT of an N-point sequence $\{x(n)\}$ is defined by

$$
X(k)=\sum_{n=0}^{N-I} x(n) W_{N}^{n k} \quad \text { for } 0 \leq k<N-1 \quad \text { and } \quad W_{N}^{j}=e^{-2 \pi j / N}
$$

and its parallel form can be represented by the vector-matrix equation as

$$
\left[\begin{array}{c}
X(0) \\
X(1) \\
\cdots \\
X(N-1)
\end{array}\right]=\left[\begin{array}{cccc}
W_{N}^{0} & W_{N}^{1} & \cdots & W_{N}^{N-1} \\
W_{N}^{0} & W_{N}^{2} & \cdots & W_{N}^{2(N-1)} \\
\cdots & \cdots & \cdots & \cdots \\
W_{N}^{0} & W_{N}^{3} & \cdots & W_{N}^{(N-1)(N-1)}
\end{array}\right]\left[\begin{array}{c}
x(0) \\
x(1) \\
\cdots \\
x(N-1)
\end{array}\right]
$$


or

$$
\underline{X}=W_{N} \times \underline{x}
$$

The structure of the FFT will be based on factorization of the twiddle factor matrix $W_{N}$. For the $\mathrm{BI} / \mathrm{LO}$ FFT, $W_{N}$ is factorized from the right first by the bit-reverse matrix $P_{b r}$ as

$$
W_{N}=W_{N}^{\prime} \times P_{b r}
$$

For the LI/BO FFT, $W_{N}$ is factorized from the left, first as

$$
W_{N}=P_{b r} \times W_{N}^{\prime \prime}
$$

By recursively decomposing the twiddle factor matrix $W_{N}^{\prime}$, the vector-matrix form of the 1-D BI/LO FFT with the length $N=2^{s}$ can be derived as follows

$$
\begin{aligned}
\underline{X} & =F G_{s}(B I(s)) \times F G_{s-1}(B I(s-1)) \times \ldots \times F G_{I}(B I(1)) \times P_{b r} \times \underline{x} \\
& =f f t{ }^{\prime} \times \underline{x}_{b}
\end{aligned}
$$

where $X$ and $x_{b}$, respectively, denote the $\mathrm{N}$-point linear output vector and $\mathrm{N}$-point bitreverse input vector (see "1-D Data Sequence: Linear and Digit-reverse" on page 21). The matrix $F G_{k}(B I(k))$ denotes the k-th radix-2 butterfly stage of the BI/LO FFT. The chapter will show the further partition of the butterfly stage matrix into three matrices as

$$
F G_{k}(B I(k))=P_{l k} \times B I(k) \times P_{r k}
$$

Some essential physical meaning in FFT algorithm implementation can be found through the three-matrix representation of the butterfly stage. The right permutation matrix $P_{r k}$ and the left permutation matrix $P_{l k}$ can specify the input data sequence and the output data sequence of the stage, respectively. The center block diagonal matrix $B I(k)$ performs the radix-2 butterfly operations of the k-th stage and specifies the twiddle 
factor sequence. It is defined as

$$
B I(k)=\left[\begin{array}{cccc}
b i_{k}(0) & 0 & \ldots & 0 \\
0 & b i_{k}(l) & \ldots & 0 \\
\ldots & \ldots & \ldots & \ldots \\
0 & 0 & \ldots b i_{k}(N / 2-1)
\end{array}\right]
$$

where " 0 " is a 2 by 2 zero matrix and the radix- 2 butterfly module $b i_{k}(n)$ along the diagonal of $B I(k)$ is defined as

$$
b i_{k}(n)=\left[\begin{array}{cc}
W_{N}^{0} & W_{N}^{i} \\
W_{N}^{0} & -W_{N}^{i}
\end{array}\right] \quad \text { with } i=\frac{N}{2^{k}} \times \operatorname{Int}\left(\frac{n}{2^{s-k}}\right)
$$

The function $\operatorname{Int}(x)$ denotes the integer part of the real number x. $P_{r k}$ specifies the interconnection between inputs and butterfly modules and is an $\mathrm{N}$ by $\mathrm{N}$ permutation matrix with its elements defined as

$$
P_{r k}(n, m)= \begin{cases}1 & \text { for } n=\frac{N}{2^{s+1-k}} \times \operatorname{Mod}(m)_{2^{s+1-k}}+\operatorname{Int}\left(\frac{m}{2^{s+1-k}}\right) \\ 0 & \text { otherwise }\end{cases}
$$

where $\operatorname{Mod}(x)_{y}$ denotes the modulo operation on the number $\mathrm{x}$ with modulo length $\mathrm{y}$ and is defined as

$$
\operatorname{Mod}(x)_{y}=x-\operatorname{Int}\left(\frac{x}{y}\right) \times y
$$

Similarly, $P_{l k}$ specifies the interconnection between outputs and butterfly modules with its elements defined as 


$$
P_{l k}(n, m)= \begin{cases}1 & \text { for } m=\frac{N}{2^{s+1-k}} \times \operatorname{Mod}(n)_{2^{s+1-k}}+\operatorname{Int}\left(\frac{n}{2^{s+1-k}}\right) \\ 0 & \text { otherwise }\end{cases}
$$

The bit-reverse matrix is also an $\mathrm{N}$ by $\mathrm{N}$ permutation matrix with its elements defined as

$$
P_{b k}(n, m)= \begin{cases}1 & \text { for } n=b r(m) \\ 0 & \text { otherwise }\end{cases}
$$

where $b r(m)$ denotes the bit-reverse operation on the index $m$ with the number of bits $s$ as follows:

$$
\operatorname{br}(m)_{s}=\operatorname{br}\left(m_{s-1} m_{s-2} \ldots m_{1} m_{0}\right)_{s}=m_{0} m_{1} \ldots m_{s-2} m_{s-1}
$$

\section{1-D Linear Inputs And Bit-reverse Outputs FFT}

The vector-matrix form of the 1-D LI/BO FFT with the length $\mathrm{N}=2^{\mathrm{S}}$ can be obtained by further factoring the twiddle factor matrix $W_{N}^{\prime \prime}$ in Equation (4.3b) as follows:

$$
\underline{X}=P_{b r} \times F G_{l}(B L(s)) \times F G_{2}(B L(s-1)) \times \ldots \times F G_{s}(B L(1)) \times \underline{x}
$$

Multiplying $P_{b r}$ to both sides of Equation (4.13), we have

$$
\underline{X}_{b}=P_{b r} \times \underline{X}=f f t^{\prime \prime} \times \underline{x}
$$

As the BI/LO FFT case, the k-th butterfly stage of the LI/BO FFT can be further decomposed into three cascaded matrices

$$
F G_{(s+1-k)}(B L(k))=P_{l(s+1-k)} \times B L(k) \times P_{r(s+1-k)}
$$

$B L(k)$ is the butterfly operation matrix of the $\mathrm{k}$-th stage. Its input interconnection matrix is represented by $P_{r(s+1-k)}$ and its output interconnection matrix is $P_{l(s+l-k)}$. The fundamental difference between the $\mathrm{BI} / \mathrm{LO}$ and $\mathrm{LI} / \mathrm{BO}$ structures is the definition of the butterfly 
operation matrix. The butterfly operation matrix for the LI/BO FFT is defined as

$$
B L(k)=\left[\begin{array}{cclc}
b l_{k}(0) & 0 & \ldots & 0 \\
0 & b l_{k}(l) & \ldots & 0 \\
\ldots & \ldots & \ldots & \ldots \\
0 & 0 & \ldots & b l_{k}(N / 2-1)
\end{array}\right]
$$

The radix- 2 butterfly module $b l_{k}(n)$ of $B L(k)$ is defined as

$$
b l_{k}(n)=\left[\begin{array}{cc}
W_{N}^{0} & W_{N}^{i} \\
W_{N}^{0} & -W_{N}^{i}
\end{array}\right] \quad \text { with } i=\operatorname{br}\left(\bmod (n)_{2^{k-1}}\right)_{s-1}
$$

\section{Equivalence of FFT Algorithms by Matrix Transformation}

The previous two sections have shown that each stage of $\mathrm{BI} / \mathrm{LO}$ and $\mathrm{LI} / \mathrm{BO}$ FFT algorithms can be represented by three cascaded matrices. In software or hardware realization of the FFT, these matrices can represent input, output and twiddle factor addressing sequences. In this section, some equivalent relationship of these matrices will be shown. Through these equivalent algorithms, it can be seen that one kind of FFT structure can be derived from the other kind of the FFT structure. After transformation, the new three cascaded matrices also denote the three addressing sequences for the FFT stage. The last two sections employ these equivalent relationships to M-D FFT. Some salient results can be obtained such as the unified 1-D FFT addressing sequence to implement the M-D FFT.

The following will list the theorems that describe the equivalent relationship of the input data, output data, bit-reverse and the twiddle factor matrices [Ju, 1993].

Theorem 1: (Input Interconnection Operation) 


$$
P_{r\left(\operatorname{Mmod}(i+j-1)_{s}\right)}=P_{r i} \times P_{r j} \quad \text { for } 1 \leq i \leq s \text { and } 1 \leq j \leq s
$$

where Mmod(x) is a modified modulo operation function defined as

$$
\operatorname{Mmod}(x)_{y}=x-\operatorname{Int}\left(\frac{x-1}{y}\right) \times y
$$

Theorem 2: (Output Interconnection Operation)

$$
P_{l\left(\operatorname{Mmod}(i+j-1)_{s}\right)}=P_{l i} \times P_{l j} \quad \text { for } \quad l \leq i \leq s \text { and } 1 \leq j \leq s
$$

Theorem 3: (Input and Output Interconnection Equivalent)

$$
P_{r k}=P_{l(s+2-k)} \text { and } P_{l k}=P_{r(s+2-k)}
$$

Theorem 4: (Bit-Reverse Equivalent)

$$
P_{l k}=P_{b r} \times P_{r k} \times P_{b r} \text { and } P_{r k}=P_{b r} \times P_{l k} \times P_{b r}
$$

Theorem 5: (Equivalence between BI/LO and LI/BO Butterfly Matrices)

$$
B I(k)=P_{r s} \times P_{b r} \times B L(k) \times P_{b r} \times P_{l s}
$$

and

$$
B L(k)=P_{r s} \times P_{b r} \times B I(k) \times P_{b r} \times P_{l s}
$$

A variety of FFT algorithms can be obtained by changing the order of the input and the output data sequences or by changing the order of butterfly matrix computations. Two examples are given in the following.

\section{A. Example of Deriving Constant Geometry FFT from In-Place FFT.}

The vector-matrix form of a 16-point in-place LI/BO FFT can be obtained from Equation (4.13) and (4.15) and expressed as

$$
\begin{aligned}
P_{b r} \times \underline{X}=P_{l 1} \times & B L(4) \times P_{r 1} \times P_{l 2} \times B L(3) \times P_{r 2} \times P_{l 3} \\
& \times B L(2) \times P_{r 3} \times P_{l 4} \times B L(1) \times P_{r 4} \times \underline{x}
\end{aligned}
$$


It can be derived from Theorem 1, 2 and 3 that

$$
P_{r 1} \times P_{l 2}=P_{r 2} \times P_{l 3}=P_{r 3} \times P_{l 4}=P_{r 4} \times P_{l 1}=P_{r 4}
$$

Thus, Equation (4.24) becomes

$$
\begin{aligned}
P_{b r} \times \underline{X}=P_{l 1} \times & B L(4) \times P_{r 4} \times P_{l l} \times B L(3) \times P_{r 4} \times P_{l 1} \\
& \times B L(2) \times P_{r 4} \times P_{l 1} \times B L(1) \times P_{r 4} \times \underline{x}
\end{aligned}
$$

Equation (4.26) represents constant geometry 16-point LI/BO FFT. Similarly, constant geometry BI/LO FFTs can be derived from in-place BI/LO FFTs.

\section{B. Example of Deriving In-Place LI/BO FFT from In-Place BI/LO FFT}

The vector-matrix form of a 16-point in-place BI/LO FFT can be obtained from Equations (4.4) and (4.5) and represented by

$$
\begin{aligned}
\underline{X}= & P_{l 4} \times B I(4) \times P_{r 4} \times P_{l 3} \times B I(3) \times P_{r 3} \times P_{l 2} \\
& \times B I(2) \times P_{r 2} \times P_{l 1} \times B I(1) \times P_{r 1} \times P_{b r} \times \underline{x}
\end{aligned}
$$

Using $B L(i)$ to replace $B I(i)$ by Equation (4.23a) and using Equations (4.19)-(4.22) to reduce the number of matrices, we can transform Equation (4.27) into

$$
\underline{X}=P_{b r} \times B L(4) \times P_{r 4} \times B L(3) \times P_{r 4} \times B L(2) \times P_{r 4} \times B L(1) \times P_{r 4} \times \underline{x}
$$

Multiplying $P_{b r}$ to both sides of Equation (4.28) and using the equivalent relationship of Equation (4.25), the equation of the 16-point in-place LI/BO FFT can be obtained as shown in Equation (4.24).

\section{Formulation of Vector Matrix Form of 2-D DFT}

For a 2-D array $\left(\mathrm{N}_{1}, \mathrm{~N}_{2}\right)$, the 2-D DFT by the row-column approach over the region is defined as 


$$
\begin{gathered}
X\left(k_{1}, k_{2}\right)=\sum_{n_{2}=0}^{N_{2}-1}\left[\sum_{n_{1}=0}^{N_{1}-1} x\left(n_{1}, n_{2}\right) W_{N_{1}}^{n_{1} k_{1}}\right] W_{N_{2}}^{n_{2} k_{2}} \\
\text { for } 0 \leq k_{1} \leq N_{1} \text { and } 0 \leq k_{2} \leq N_{2}
\end{gathered}
$$

The parallel vector-matrix form of the 2-D DFT can be expressed as

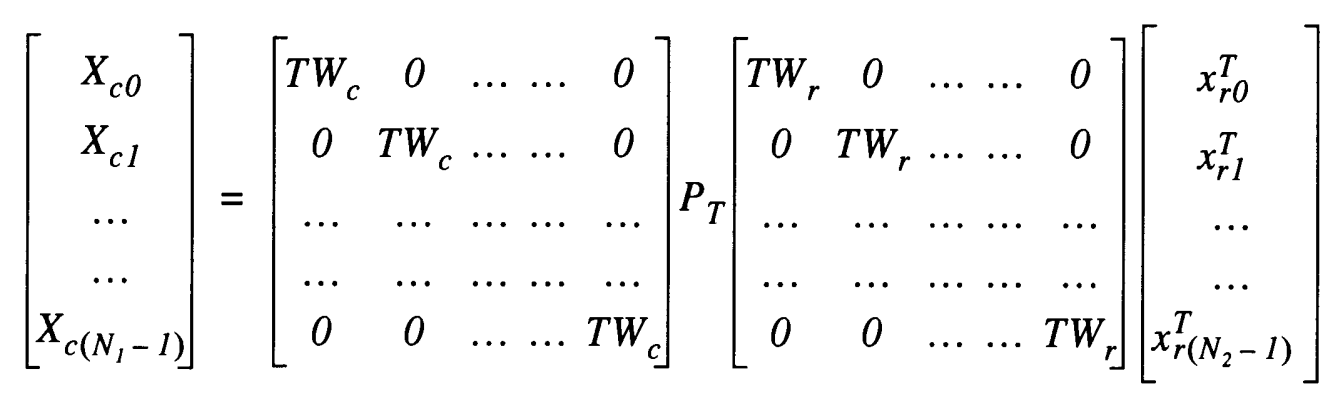

where $T W_{r}$ and $T W_{c}$ denote the twiddle factor matrices for the row DFT and column DFT, respectively. $x_{r i}$ is the $\mathrm{i}$-th row of the input array and $X_{c j}$ is the j-th column of the output array. Set the row length $N_{1}=2^{s_{1}}$, column length $N_{2}=2^{s_{2}}$, and total elements $N=N_{1} \times N_{2}$. The transpose matrix PT is employed to transform the 2-D array from row-major order to column-major order and is also a permutation matrix expressed as

$$
P_{T}=P_{r\left(s_{1}+1\right)} \quad \text { and } \quad P_{T}^{-1}=P_{l\left(s_{1}+1\right)}
$$

\section{2-D Linear Inputs And Bit-reverse Outputs FFT}

The vector-matrix form for the 2-D LI/BO FFT implementation of Equation (4.30) can be represented by 


$$
\left[\begin{array}{c}
X_{b c 0} \\
X_{b c l} \\
\ldots \\
\ldots \\
X_{b c\left(N_{1}-1\right)}
\end{array}\right]=\left[\begin{array}{ccccc}
c f f t & 0 & \ldots & \ldots & 0 \\
0 & c f f t & \ldots & \ldots & 0 \\
\ldots & \ldots & \ldots & \ldots & \ldots \\
\ldots & \ldots & \ldots & \ldots & \ldots \\
0 & 0 & \ldots & \ldots & c f f t
\end{array}\right] P_{T}\left[\begin{array}{ccccc}
r f f t & 0 & \ldots & \ldots & 0 \\
0 & r f f t & \ldots & \ldots & 0 \\
\ldots & \ldots & \ldots & \ldots & \ldots \\
\ldots & \ldots & \ldots & \ldots & \ldots \\
0 & 0 & \ldots & \ldots & r f f t
\end{array}\right]\left[\begin{array}{c}
x_{r 0}^{T} \\
x_{r 1}^{T} \\
\ldots \\
\ldots \\
x_{r\left(N_{2}-1\right)}^{T}
\end{array}\right]
$$

where $X_{b c i}$ is the i-th column of the 2-D bit-reverse output array. rfft and cfft can be represented by Equation (4.13) with length $N_{l}$ and $N_{2}$, respectively. It can be derived that the $N_{2} r f f t s$ can be implemented by the first $s_{l}$ stages of the N-point 1-D FFT and the $N_{l}$ $c f f t s$ by the first $s_{2}$ stages of the N-point 1-D FFT. Thus, Equation (4.32) yields

$$
\begin{aligned}
\underline{X}_{b c}= & F G_{1}\left(B L\left(s_{2}\right)\right) \times F G_{2}\left(B L\left(s_{2}-1\right)\right) \times \ldots \times F G_{s_{2}}(B L(1)) \times P_{T} \\
& \times F G_{1}\left(B L\left(s_{1}\right)\right) \times F G_{2}\left(B L\left(s_{1}-1\right)\right) \times \ldots \times F G_{s_{1}}(B L(1)) \times \underline{x}_{r}^{T}
\end{aligned}
$$

Where $\underline{X}_{b c}$ is the N-point output vector and $\underline{x}_{r}^{T}$ is the N-point input vector shown in Equation (4.32). Combining the transpose matrix PT with the row FFTs and using Theorem 1, 2 and 3, Equation (4.33) can be transformed into a new form of the 2-D FFT structure

$$
\begin{array}{r}
\underline{X}_{b c}=F G_{1}\left(B L\left(s_{2}\right)\right) \times F G_{2}\left(B L\left(s_{2}-1\right)\right) \times \ldots \times F G_{s_{2}}(B L(1)) \\
\times F G_{s_{2}+1}\left(B L\left(s_{1}\right)\right) \times F G_{s_{2}+2}\left(B L\left(s_{1}-1\right)\right) \\
\times \ldots \times F G_{s_{1}+s_{2}}(B L(1)) \times x_{c}
\end{array}
$$

where $x_{c}$ is an $\mathrm{N}$-point vector in the linear column-major order of the input array.

Comparing Equation (4.34) with Equation (4.13) and setting $s=s_{1}+s_{2}$, we can see that the $N_{l}$ by $N_{2}$ 2-D FFT has the same interconnection (butterfly) structure as the N-point 1-D FFT. In another word, they can have the same Signal Flow Graph structure. Figure 14 shows the SFG structure of the traditional row-column 4 by 4 2-D LI/BO FFT implementation. Figure 15 shows the LI/BO SFG structure of the 4 by 4 mapped 2-D FFT 
and 16-point 1-D FFT. The top indexing represents the inputs, outputs and twiddle factors for the 2-D case and the bottom indexing is for the 1-D case. The index "i" shown in the figure denotes the twiddle factor $W_{16}^{i}$. It can be seen that the twiddle factor matrices are the same for the k-th stage of the row FFT, column FFT and 1-D FFT. The twiddle factor addressing sequence can be obtained from Equation (4.17). The input and the output interconnections (butterfly) are the same for the 1-D and 2-D FFTs with the same number of points and the addressing sequences can be obtained from Equations (4.8) and (4.10).

\section{2-D Bit-reverse Inputs And Linear Outputs FFT}

The vector-matrix form of the BI/LO 2-D FFT can be derived in a similar way to that of the LI/BO 2-D FFT. If the $T W_{r}$ and $T W_{c}$ of Equation (4.30) are implemented by Equation (4.4), then Equation (4.30) can be transformed into the following form

$$
\begin{aligned}
& \underline{X}_{c}=F G_{s_{2}}\left(B I\left(s_{2}\right)\right) \times F G_{s_{2}-1}\left(B I\left(s_{2}-1\right)\right) \times \ldots \times F G_{1}(B L(1)) \times P_{T} \\
& \quad \times F G_{s_{I}}\left(B L\left(s_{1}\right)\right) \times F G_{s_{1}-1}\left(B L\left(s_{1}-1\right)\right) \times \ldots \times F G_{1}(B L(1)) \times x b r
\end{aligned}
$$

where $\underline{X}_{c}$ is an N-point vector in the column-major order of the linear output array and $x b_{r}$ is an N-point vector in the row-major order of the bit-reverse input array. Combining the transpose matrix $\mathrm{P}_{\mathrm{T}}$ with the column FFTs and using Theorem 1, 2 and 3, Equation (4.35) can become

$$
\begin{aligned}
\underline{X}_{r}^{T}= & F G_{s_{1}+s_{2}}\left(B I\left(s_{2}\right)\right) \times F G_{s_{I}+s_{2}-1}\left(B I\left(s_{2}-1\right)\right) \times \ldots \times F G_{s_{I}+1}(B L(1)) \\
& \times F G_{s_{1}}\left(B L\left(s_{1}\right)\right) \times F G_{s_{1}-1}\left(B L\left(s_{1}-1\right)\right) \times \ldots \times F G_{l}(B L(I)) \times x_{b r}^{T}
\end{aligned}
$$

where $\underline{X}^{T}$ is an N-point vector in the row-major order of the linear output array. As the LI/BO case by comparing Equation (4.36) with Equation (4.4), the $N_{l}$ by $N_{2} 2$-D FFT has 
the same interconnection structure as the N-point 1-D FFT. Moreover, the butterfly operation matrices are the same for the k-th stage of the row FFT, column FFT and 1-D FFT. Figure 16 shows the SFG structure of the traditional row-column 4 by $42-\mathrm{D} \mathrm{BI} / \mathrm{LO}$ FFT implementation. Figure 17 shows the LI/BO SFG structure of the 4 by 4 mapped 2D FFT and 16-point FFT.

\section{2-D FFT}

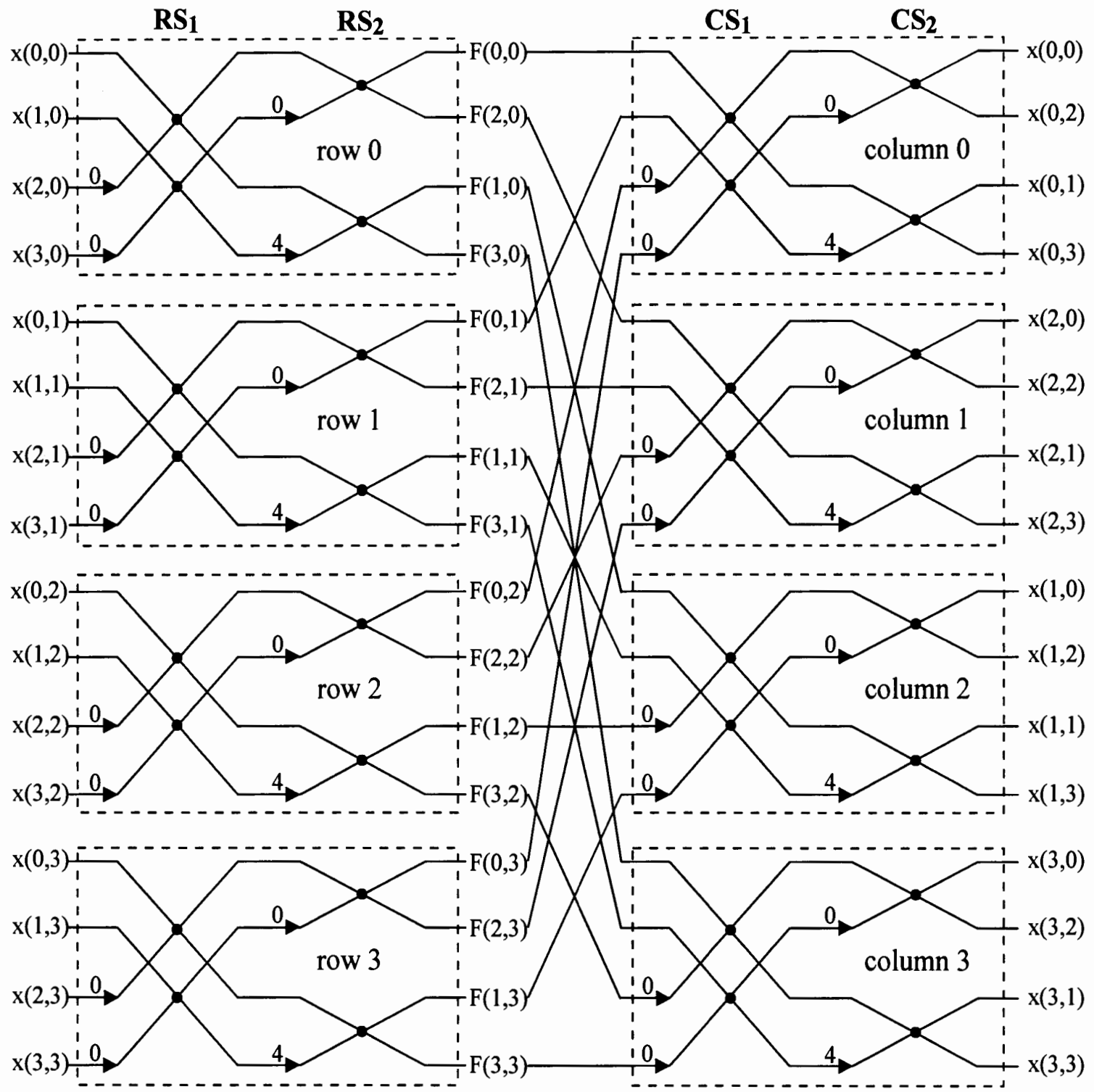

Eigure 14. Signal Flow Graph of Traditional LI/BO 4 by 4 2-D FFT 


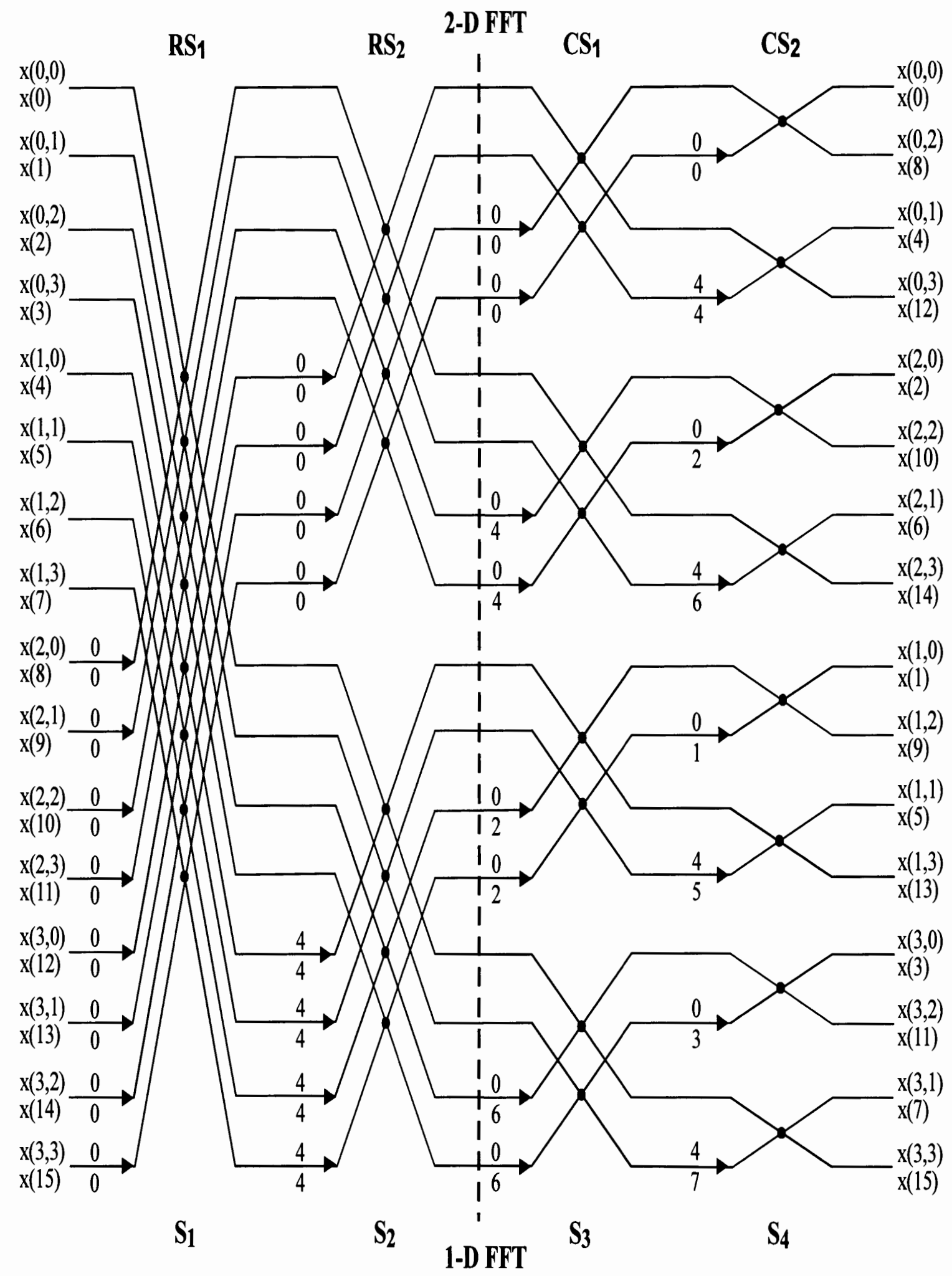

Figure 15. Signal Flow Graph of LI/BO 4 by 4 2-D Mapped \& 16-point 1-D FFTs 


\section{2-DFFT}

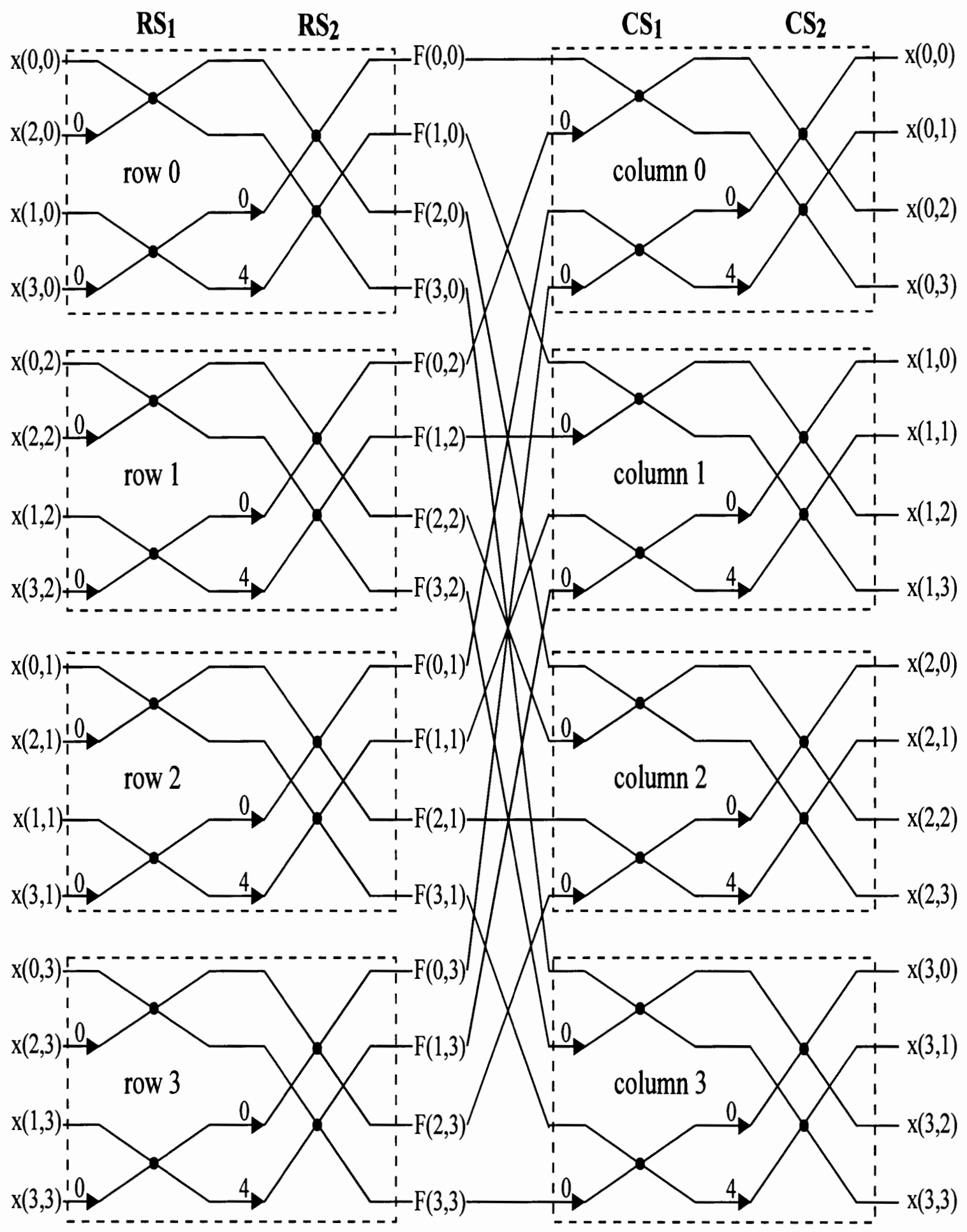

Figure 16. Signal Flow Graph of Traditional BI/LO 4 by 4 2-D FFT 


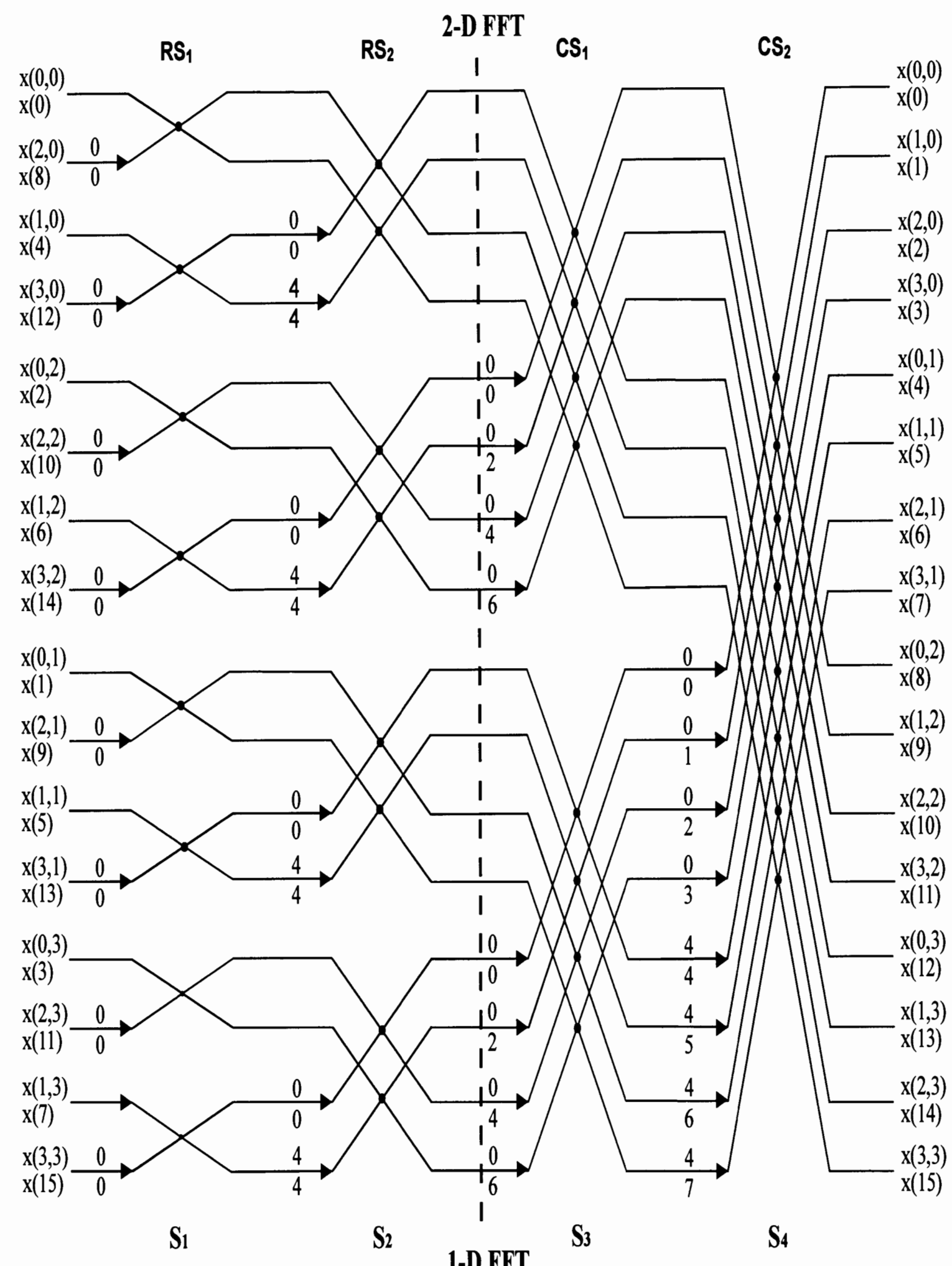

Eigure 17. Signal Flow Graph of BI/LO 4 by 4 2-D Mapped \& 16-point 1-D FFTs 


\section{CHAPTER V}

\section{WINDSP: A BLOCK ORIENTED WINDOW-BASED DSP ENVIRONMENT}

WinDSP is a comprehensive software development system which was developed by the author for Sharp LH9124/LH9320 DSP chips. WinDSP is an integrated software development environment for information processing and data visualization executing under Microsoft Windows. It is also intended to manage the complete development of real-time DSP applications, from conceptualization and prototyping, to implementation in real-time hardware. WinDSP components include visual function-level representation of LH9124 instruction set, an interactive user application development editor (see Figure 18), an external LH9320 address generator package (see Figure 20), and a text/graphics data display window. Although the software is not able to perform real-time simulations, the user can still analyze data for real-time algorithms in real-time environment and in nearly real-time mode. It is also intended to ease code development for architectures that are not easy targets for conventional compilers. WinDSP supports users with varying levels of sophistication. The algorithm designer with little knowledge (or interest) in realtime DSP architectures can quickly and easily produce a simulation or real-time prototype using function blocks from the simulator. The program is also intended to minimize the system design turn-around time and eliminate the time-consuming software coding and debugging. This software is designed to be targetable in two ways. First, it can 
synthesize FFT algorithms for a variety of array signal architectures, where the user specifies the function block diagram of the architecture. Second, it can target custom system configuration development in which the function block features are next inherited by the system application design. At the highest level, an algorithm is described using a hierarchical block diagram. Once the application is partitioned into the components of the function block diagram, the function-level representation of instructions will be directly mapped from the function block diagram to the system application. At the lowest level, the user can either simulate the algorithm, simulate the target architecture or download the instruction code and parameters from the simulator to the hardware system for real-time simulation.

A number of other block-diagram systems for DSP have appeared in recent years. Four commercially available programs are BOSS [Shanmugan, 1987] from the University of Kansas, DSPlay from Burr-Brown, Signal Processing Worksystem (SPW) from Comdisco and the Hypersignal-DSP from Hyperception. Hypersignal-DSP supports both real-time and non-real-time types of DSP. Hypersignal-DSP provides virtually limitless simulation, analysis, design, and support for a number of optional plug-in DSP/acqusition hardware cards to what is referred to as "associated Real-Time Signal Processing." BOSS and SPW, on the other hand, were both strictly simulation systems, not aimed at real-time implementations. BOSS is particularly distinguished for its project management facilities. DSPlay is a PC-based system for both simulation and code generation (for the AT\&T DSP32). All the systems could benefit from using the Unified Indexing of FFT Algorithms technique presented in Chapter IV. 
Function block diagrams for simulation actually have a long and distinguished history, testifying to their attractiveness to the DSP community. A number of systems were proposed or built in the 1960's [Dertouzous, 1969], [Gold and Rader, 1969] and 1970's [Crystal and Kulsrud, 1974], [Korn, 1977]. The object-oriented approach applied in them makes these systems easy to simulate finite register-length effects; WinDSP relies on a simulation of the target hardware to accomplish the same objective. All of the systems are aimed at simulation, not real-time implementation, and most perform the scheduling at run-time, which is usually not practical in real-time implementations. The discrete Fourier transform is widely used for digital filtering and spectrum analysis, the effects of finite register length in DFT calculations are important. In the case of digital filters, however, a precise analysis is useful for estimates of the effect of arithmetic roundoff. Often a simplified analysis based on modifying the results by truncation is sufficient for the purpose of choosing the required register length for computing the Fourier transform.

\section{The WinDSP Model}

Graphical representation of computer program is a controversial topic. For general-purpose programming, many experimental systems have been disappointing. An example is Pict [Glinert and Tanimoto, 1984], a visual programming system in which entire programs are built graphically. However, many such systems go to the extreme in an attempt to express everything graphically, resulting in complexity limitations, cryptic and cumbersome representations for user and an arbitrary selection of symbols to 
represent concepts that have no natural visualization. WinDSP software, depicted in Figure 18, uses graphical icons to represent the high-level structure of the program, leaving many details to programming of salient features. These features allow easy setup of the built-in instruction set and data flow configurations. The graphical representation of the data flow organization is simply a line linking two graphical icons on the screen of the program. The author makes no claim that a graphical representation of this type is suitable for all applications, however, the author is deeply convinced that this approach is suitable for applications that use the entire LH9124/LH9320 chip set functions.

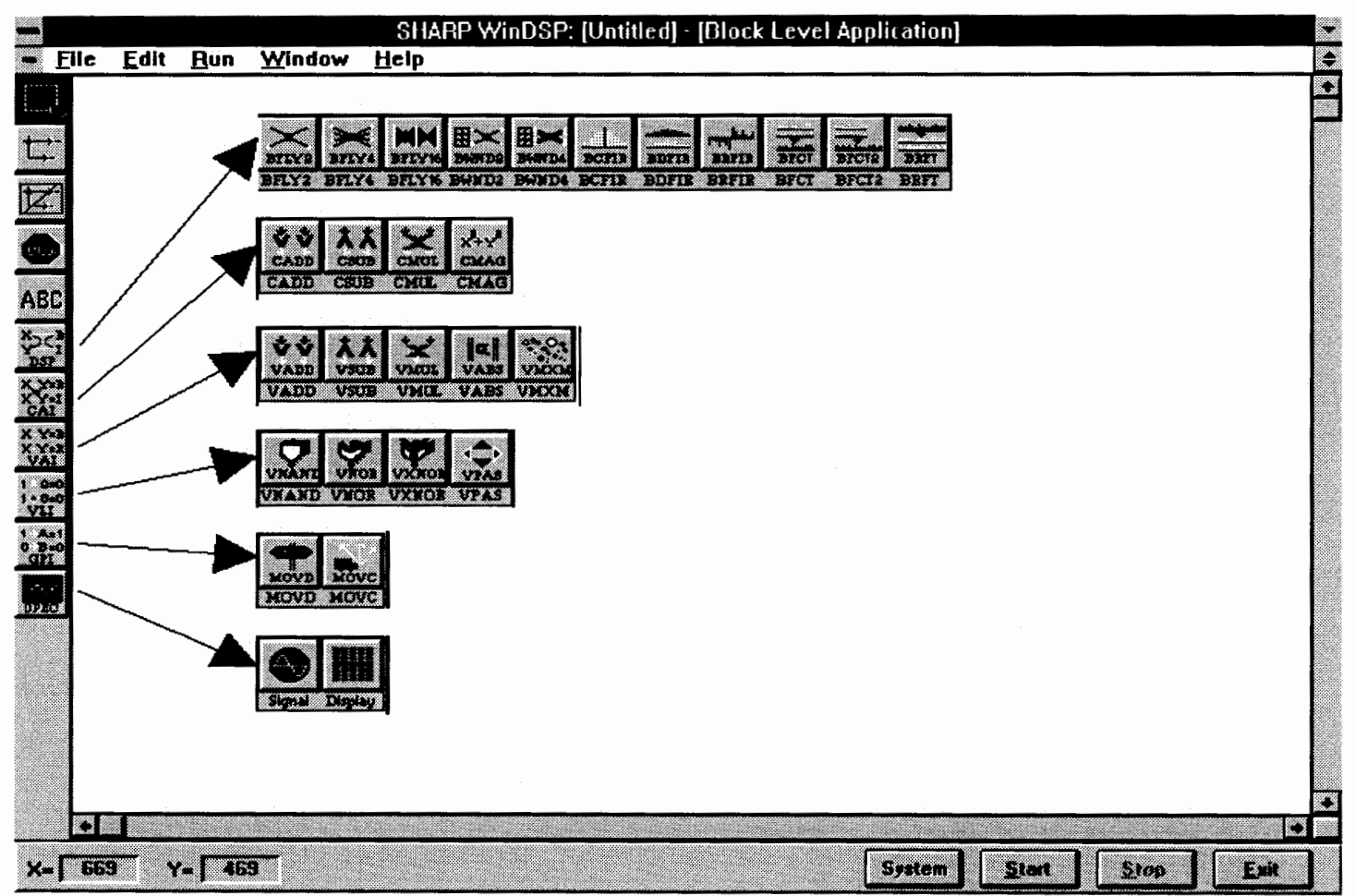

Figure 18. Graphical Icons of WinDSP Software.

There are 27 graphical icons in WinDSP software shown in Figure 18. Each graphical icons represents the function-level representation of LH9124 instruction set. There are five categories of LH9124 instructions set (see "The LH9124 Instruction Set" 
on page 35). The first fly-out menu, starting at the top, consists of function-level representation of LH9124 instruction set for DSP categories. The second fly-out menu consists of function-level representation for the CAI categories. The third fly-out menu consists of function-level representation for VLI categories. The fourth fly-out menu shows the GPI categories function-level representation. The last fly-out menu consists of the signal generator function and text/graphics display function. The signal generator function allows the user to create sample data for the application algorithms and the text/ graphics display function displays the input and output data either in spectrum graphics or hexadecimal value.

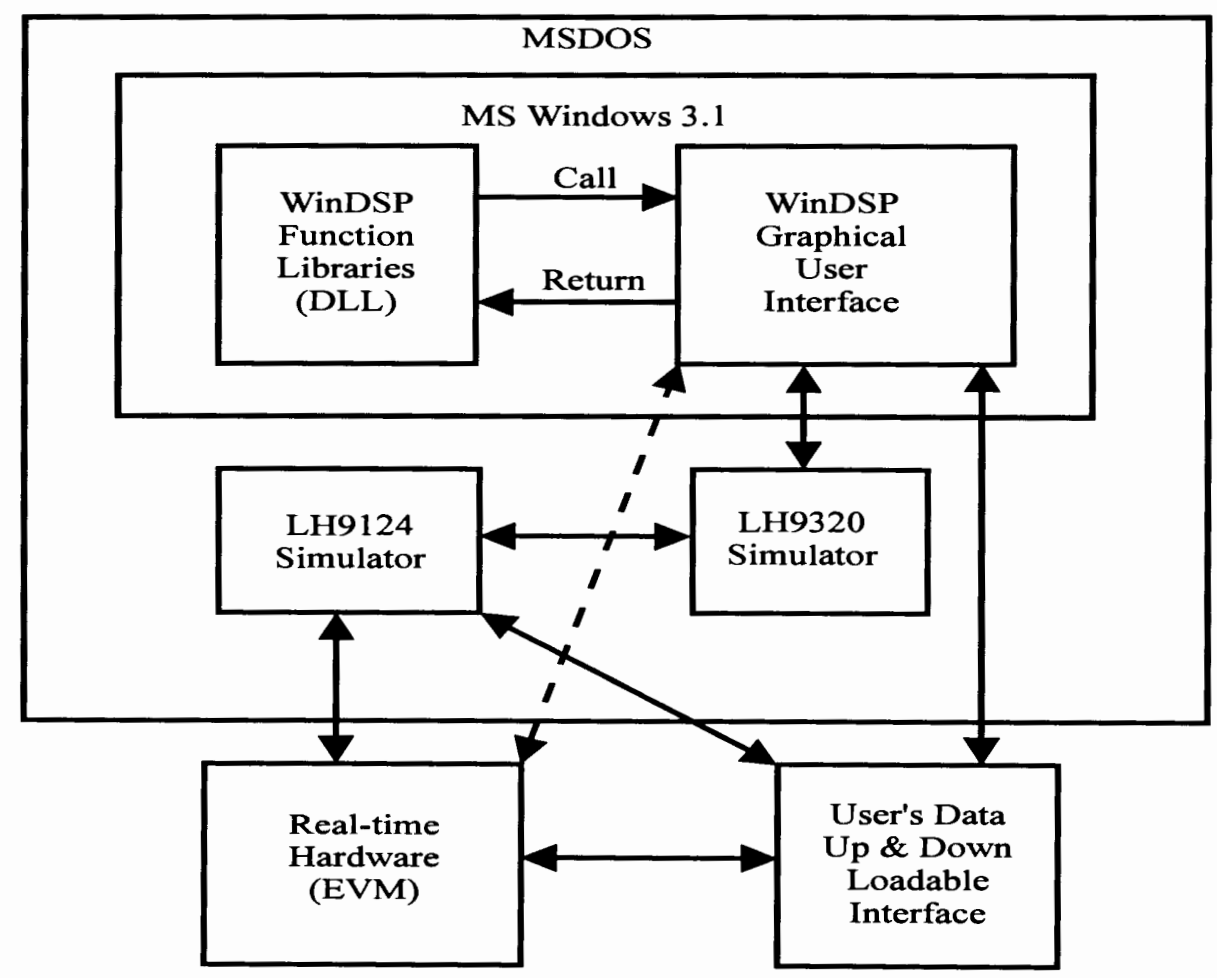

Eigure 12 The WinDSP Software Architecture

The WinDSP software architecture is depicted schematically in Figure 19, was developed primarily to implement unified indexing technique and help demonstrate the 
equivalent relationship of function-level representation and the Unified Indexing of FFT algorithms (see CHAPTER IV on page 49). On the other hand, the WinDSP is used to improve productivity, promote software reuse in a wide variety of application domains and facilitate multidisciplinary problem solving.

In Figure 19, the function instructions of the LH9124 DSP are the core of the WinDSP simulation. These function instructions are coded as Dynamic Link Libraries (DLL) or WinDSP function libraries. When WinDSP calls for an instruction, the function library of that instruction is loaded into the program and released immediately when the instruction is no longer required by the main program (i.e the WinDSP Graphical User Interface module). This frees up the computer memory. The WinDSP Graphical User Interface module (see Figure 18), acts as a program manager to handle communication with all the other modules. The LH9124 simulator module and the LH9320 address generator simulator module were first developed running under Disk Operating System (DOS) environment. The LH9124 DOS simulator software simulates the functional behavior of LH9124 Digital Signal Processor's entire instruction set. The LH9320 DOS simulator is a menu-driven software that is used in conjunction with the LH9124 simulator. It generates address patterns and displays or prints the resulting addressing sequence values. The WinDSP software uses the LH9320 DOS simulator to generate the addresses for its simulation. The setup and configuration of the LH9124's signal parameters from the WinDSP can be ported to the Evaluation Module Board (EVM). Lastly, users upload and download module allows users to upload external sample data for analysis and evaluation, as well as download simulated data to their hardware system. 
WinDSP also simulates the LH9124 DSP chip's characteristics and have functionality similar to the LH9124 DOS simulator, except WinDSP is Windows-based Graphics User Interface and easy to use. Figure 20 shows the initial start-up menu of the LH9320 DOS simulator when executed. There are seven selections in that start-up menu: (1) Edit program memory, (2) Edit channel length/overlap memory, (3) Edit configuration memory, (4) Execute the setup memory, (5) Display/print the address memory, (6) Save/ load setup memory, and (7) Return to DOS (Exit the program). Since the LH9320 is not the main focus of the thesis, the author makes no attempt to discuss in detail the function of each selection. Further discussion of LH9320 DOS simulator software can be found in SHARP LH9320 RTS User’s Guide.

LH9320 ADDRESS GENERATOR SIMULATOR

LH9320 RTS MEMORY MAP

1. EDIT PROGRAM MEMORY

2. EDIT CHANMEL LENGTH/OUERLAP MEMORY

3. EDIT CONF IGURAT ION MEMORY

4. EXECUTE THE SETUP MEMORY

5. DISPLAY/PR INT THE ADDRESS MEMORY

6. SAUE/LOAD SETUP MEMORY

7. RETURN TO WINDOWS

(ONE MEGABYTES UERSION)

[ Click mouse on selection box or Press number for selection ]

Figure 20. LH9320 Simulator Memory Map Menu

WinDSP software provides two levels of simulation which are Block-Level 
simulation and System-Level simulation. The Block-Level simulation, known as lowlevel simulation, is used mostly for algorithmic applications analysis, whereas the System-Level simulation (high-level) allows the user to map simulated block diagrams to a system application. Thus, WinDSP can be used for both system application development and algorithm development.

\section{Equivalent Relationship of the Function-Level Representation}

The instructions of the LH9124 are defined with a function-level representation. There are two salient features for the function-level representation of instructions. First, the instructions can be obtained directly from the algorithm to instruction mapping. Second, the instructions are optimized in the function-level representation. Therefore, users can easily use the chip set to obtain the optimum performance for their applications.

From the previous chapter, the FFT algorithm is represented by the general vectormatrix form. The 1-D FFT with length $\mathrm{N}=2^{\mathrm{s}}$ is derived as

$$
\underline{X}=F G_{s}(B S(s)) \times F G_{s-1}(B S(s-1)) \times \ldots \times F G_{l}(B S(1)) \times \underline{x}_{b}
$$

and the 2-D FFT with length $\mathrm{N}_{1}$ by $\mathrm{N}_{2}$ is derived as

$$
\begin{aligned}
\underline{X}_{r}^{T}= & F G_{s_{1}+s_{2}}\left(B S\left(s_{2}\right)\right) \times \ldots \times F G_{s_{1}+l}(B S(l)) \\
& \times F G_{s_{1}}\left(B S\left(s_{1}\right)\right) \times \ldots \times F G_{l}(B S(l)) \times \underline{x}_{b r}^{T}
\end{aligned}
$$

The mapping relationship of Equation (5.1) and Equation (5.2) to the function-level representation of LH9124 instructions for the FFT algorithm can be further discussed and is best illustrated with 1-D 16 point FFT and 2-D 4 by 4 FFT examples. Using the Equation (5.1), the vector-matrix form for the 1-D 16-point FFT can be represented by 


$$
\underline{X}=F G_{4}[B I(4)] \times F G_{3}[B I(3)] \times F G_{2}[B I(2)] \times F G_{1}[B I(1)] \times \underline{x}_{b}
$$

and using Equation (5.2) the vector-matrix for 4 by 4 2-D FFT can be represented by

$$
\underline{X}_{r}^{T}=F G_{4}[B I(2)] \times F G_{3}[B I(1)] \times F G_{2}[B I(2)] \times F G_{1}[B I(1)] \times \underline{x}_{r}^{T}
$$

Further expansion each terms of Equation (5.3), it becomes

$$
\begin{aligned}
\underline{X}= & P_{l 4} \times B I(4) \times P_{r 4} \times P_{l 3} \times B I(3) \times P_{r 3} \times P_{l 2} \\
& \times B I(2) \times P_{r 2} \times P_{l 1} \times B I(1) \times P_{r 1} \times P_{b r} \times \underline{x}
\end{aligned}
$$

and Equation (5.4) becomes

$$
\begin{aligned}
\underline{X}_{r}^{T}= & P_{l 4} \times B I(2) \times P_{r 4} \times P_{l 3} \times B I(1) \times P_{r 3} \times P_{l 2} \\
& \times B I(2) \times P_{r 2} \times P_{l 1} \times B I(1) \times P_{r 1} \times P_{b r} \times \underline{x}_{r}^{T}
\end{aligned}
$$

Notice that the permutation matrix, $P_{l k}$ and $P_{r k}$ (where $k=1,2,3,4$ ) of Equations (5.5) and (5.6), will have the same indexing sequence (see CHAPTER IV on page 49). Appendices A and B, showed the how the unified indexing is computed for 16-point 1-D FFT and 4 by 4 2-D FFT vector matrix respectively. The computed unified indexing sequences are listed Table XVII shown in Appendix C. In Appendix B, it shows that the indexing sequences of left and right permutation matrices for 4 by 4 2-D FFT vector matrix are equivalent to 1-D FFT for the same number of point. Thus, the example has proof that all the M-D FFT indexing can be simplified and implemented by 1-D indexing.

The Signal Flow Graph of 16-point 1-D FFT and 4 by 4 2-D FFT is illustrated in Figure 17. Equations (5.3) and (5.4) show that the 16-point FFT can be factored into four cascaded stages with radix-2 butterfly (see Figure 21). Table VII lists the LH9124/ LH9320 instructions associated with computing the 16-point 1-D FFT and 4 by 4 2-D FFT. These instructions are also correspond directly to the Equations (5.5) and Equation 
(5.6). The execution instructions are BFLY2 because of radix-2 FFT algorithm is implemented. The instruction BF $2 k$ (where $k=0, \ldots, \log 2[N]-1$ ) generates the address sequences for each $(j+1)$ stage, and the indexing sequences of permutation matrices $P_{r(k+l)}$ or $P_{l(k+l)}$. The instruction TF2 $j$ (where $j=0, \ldots, \log 2[N]-1$ ) generates the twiddle factor sequences for each $(j+1)$ stage corresponding to the diagonal sequence of the butterfly operation matrices $B S(j+1)$.

Table VII: Two FFT Examples using Radix-2 with LH9124/LH9320 Instructions

\begin{tabular}{|c|c|c|c|c|c|c|c|c|}
\hline & \multicolumn{4}{|c|}{ 16-point 1-D FFT } & \multicolumn{4}{c|}{ 4 by 42-D FFT } \\
\hline Stage & Instr. & Input & Twiddle & Output & Instr. & Input & Twiddle & Output \\
\hline \hline 1 & BFLY2 & RBF0 & TF20 & BF20 & BFLY2 & RBF0 & TF20 & BF20 \\
\hline 2 & BFLY2 & BF21 & TF21 & BF21 & BFLY2 & BF21 & TF21 & BF21 \\
\hline 3 & BFLY2 & BF22 & TF22 & BF22 & BFLY2 & BF22 & TF20 & BF22 \\
\hline 4 & BFLY2 & BF23 & TF23 & BF23 & BFLY2 & BF23 & TF21 & BF23 \\
\hline
\end{tabular}

The address patterns for each instruction in Table VII is shown in Table VIII. Compare the address pattern generated by the LH9320 in Table VIII and the address pattern in Table XVII (see APPENDIX C on page 109), you will find that both address sequences are same. Thus, from Equations (5.5) and (5.6), the permutation matrix $P_{l 4}$ and $P_{r 4}$ correspond to $B F 23$. The permutation matrix $P_{l 3}$ and $P_{r 3}$ correspond to $B F 22$. The permutation matrix $P_{l 2}$ and $P_{r 2}$ correspond to BF21. The permutation matrix $P_{l 1}$ and $P_{r 1}$ correspond to $B F 20$ and finally the permutation matrix $P_{b r}$ corresponds to $R B F O$. Likewise, the vector matrix $B I(4)$ has the same pattern as $T F 23$. The vector matrix $B I(3)$, $B I(2), B I(1)$ corresponds to $T F 22, T F 21$ and $T F 20$, respectively. The vector matrix $x$ of Equation 5.5 is the input vector. Table IX shows the direct mapped relationship between 
the LH9320 instruction and permutation matrix of unified indexing.

Table VIII: Address Patterns for LH9320 Instructions

\begin{tabular}{|c|c|c|c|c|c|c|c|c|c|}
\hline Stage & \multicolumn{4}{|c|}{ 1 } & \multicolumn{2}{|c|}{ 2 } & \multicolumn{2}{|c|}{3} & \multicolumn{2}{|c|}{4} \\
\hline Index & RBF0 & TF20 & BF20 & BF21 & TF21 & BF22 & TF22 & BF23 & TF23 \\
\hline \hline 0 & 0 & 0 & 0 & 0 & 0 & 0 & 0 & 0 & 0 \\
\hline 1 & 8 & 0 & 1 & 2 & 0 & 4 & 0 & 8 & 0 \\
\hline 2 & 4 & 0 & 2 & 4 & 0 & 8 & 0 & 1 & 0 \\
\hline 3 & 12 & 0 & 3 & 6 & 4 & 12 & 0 & 9 & 4 \\
\hline 4 & 2 & 0 & 4 & 8 & 0 & 1 & 0 & 2 & 0 \\
\hline 5 & 10 & 0 & 5 & 10 & 0 & 5 & 4 & 10 & 2 \\
\hline 6 & 6 & 0 & 6 & 12 & 0 & 9 & 0 & 3 & 0 \\
\hline 7 & 14 & 0 & 7 & 14 & 4 & 13 & 4 & 11 & 6 \\
\hline 8 & 1 & 0 & 8 & 1 & 0 & 2 & 0 & 4 & 0 \\
\hline 9 & 9 & 0 & 9 & 3 & 0 & 6 & 2 & 12 & 1 \\
\hline 10 & 5 & 0 & 10 & 5 & 0 & 10 & 0 & 5 & 0 \\
\hline 11 & 13 & 0 & 11 & 7 & 4 & 14 & 2 & 13 & 5 \\
\hline 12 & 3 & 0 & 12 & 9 & 0 & 3 & 0 & 6 & 0 \\
\hline 13 & 11 & 0 & 13 & 11 & 0 & 7 & 6 & 14 & 3 \\
\hline 14 & 7 & 0 & 14 & 13 & 0 & 11 & 0 & 7 & 0 \\
\hline 15 & 15 & 0 & 15 & 15 & 4 & 15 & 6 & 15 & 7 \\
\hline
\end{tabular}

Table IX: Equivalent Relationship of Unified Indexing and LH9320 Instruction Set

\begin{tabular}{|c|c|c|c|c|c|c|c|c|}
\hline Stage & \multicolumn{3}{|c|}{ 16-point 1-D FFT } & \multicolumn{4}{c|}{ 4 by 4 2-D FFT } \\
\hline (k) & Inst. & P $_{\text {Ik }}$ & BI(k) & P $_{\text {rk }}$ & Inst. & P $_{\text {lk }}$ & BI(k) & P $_{\text {rk }}$ \\
\hline \hline 1 & BFLY2 & BF20 & TF20 & RBF0 & BFLY2 & BF20 & TF20 & RBF0 \\
\hline 2 & BFLY2 & BF21 & TF21 & BF21 & BFLY2 & BF21 & TF21 & BF21 \\
\hline 3 & BFLY2 & BF22 & TF22 & BF22 & BFLY2 & BF22 & TF20 & BF22 \\
\hline 4 & BFLY2 & BF23 & TF23 & BF23 & BFLY2 & BF23 & TF21 & BF23 \\
\hline
\end{tabular}




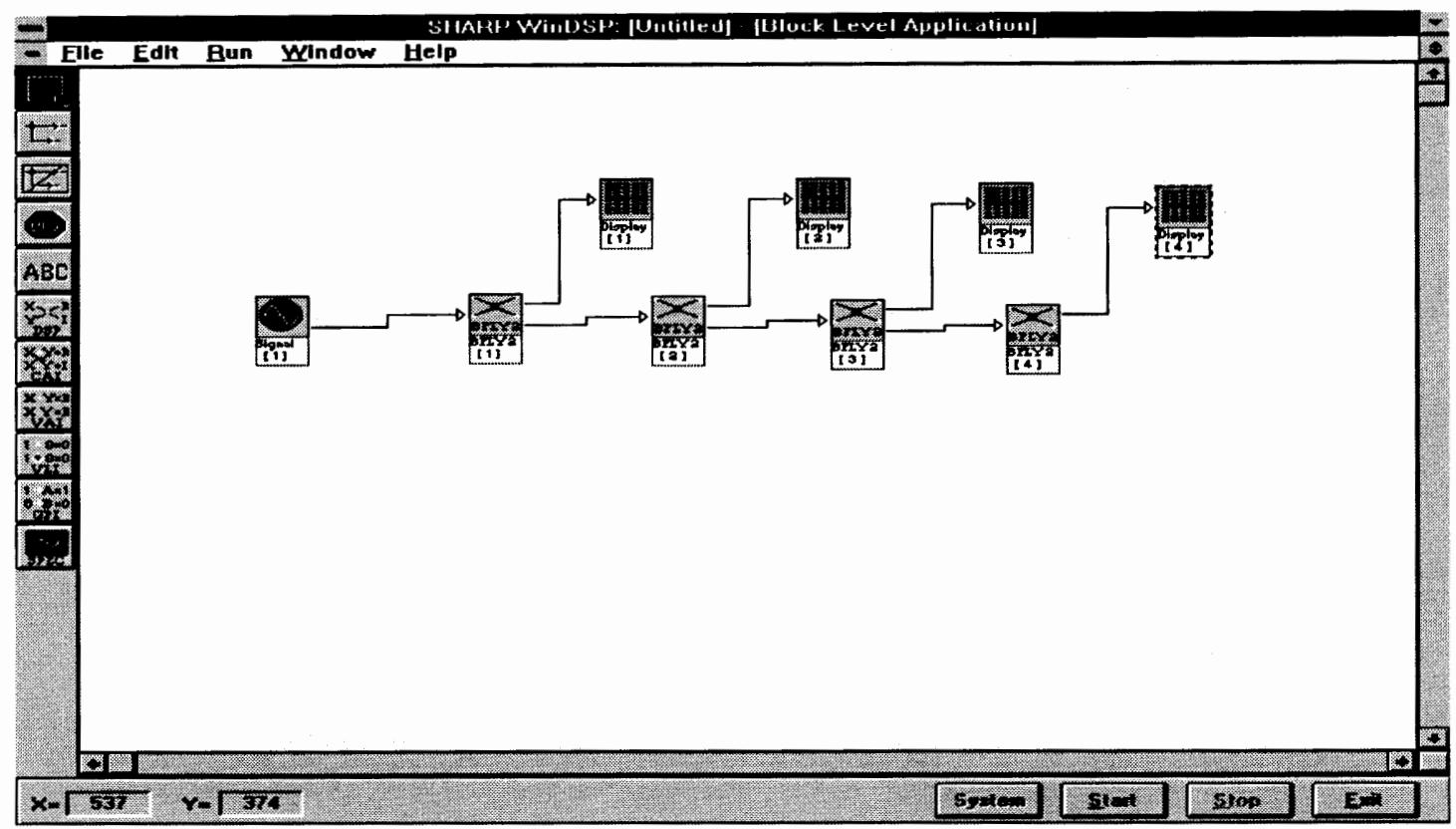

Figure 21. Function Block Diagram of 1-D 16-point \& 2-D 4 by 4 FFT Computation

\section{WinDSP Function-Level Representation Mapping}

The WinDSP has two levels for user to develop DSP applications. They are the block and system level. Once the application algorithm has been built in the block level, the system level will inherit all the configurations, parameters and setup of the LH9124 chip set. On the start-up of WinDSP, the block level working environment is default. The user starts building algorithm application at this stage. After finishing building the algorithm, the user can simulate the application and goes on to the system level working environment to build the system related application. To illustrate how WinDSP works, the 16-point 1-D FFT example showed in Table VII will be presented. As shown in Figure 21, the radix-2 icons, signal generator icon and the display icons were selected from the DSP and SPEC fly-out menu (see Figure 18). Each icon is arranged in the block 
level working environment as shown in Figure 21. A line between two icons indicates data path connection. This can be done by selecting the line menu button and drawing the line between two icons. The signal generator window is called when the left mouse button is double clicked on the signal generator icon (see Figure 22). The input sample data can be generated by selecting the signal generator menu and the external data can be loaded by selecting the load data button. An arbitrary value can be entered for the input data frequency range. The value for magnitude range, which must be less than 1 and greater or equal to -1 . In the FFT algorithm, two types of input data are needed in order to compute the algorithm. The next input data must be the coefficient data and which can be generated by using the signal generator program. The default screen of Figure 22 shows generating real data screen. The imaginary data screen can be called by clicking on the Generate Imaginary button.

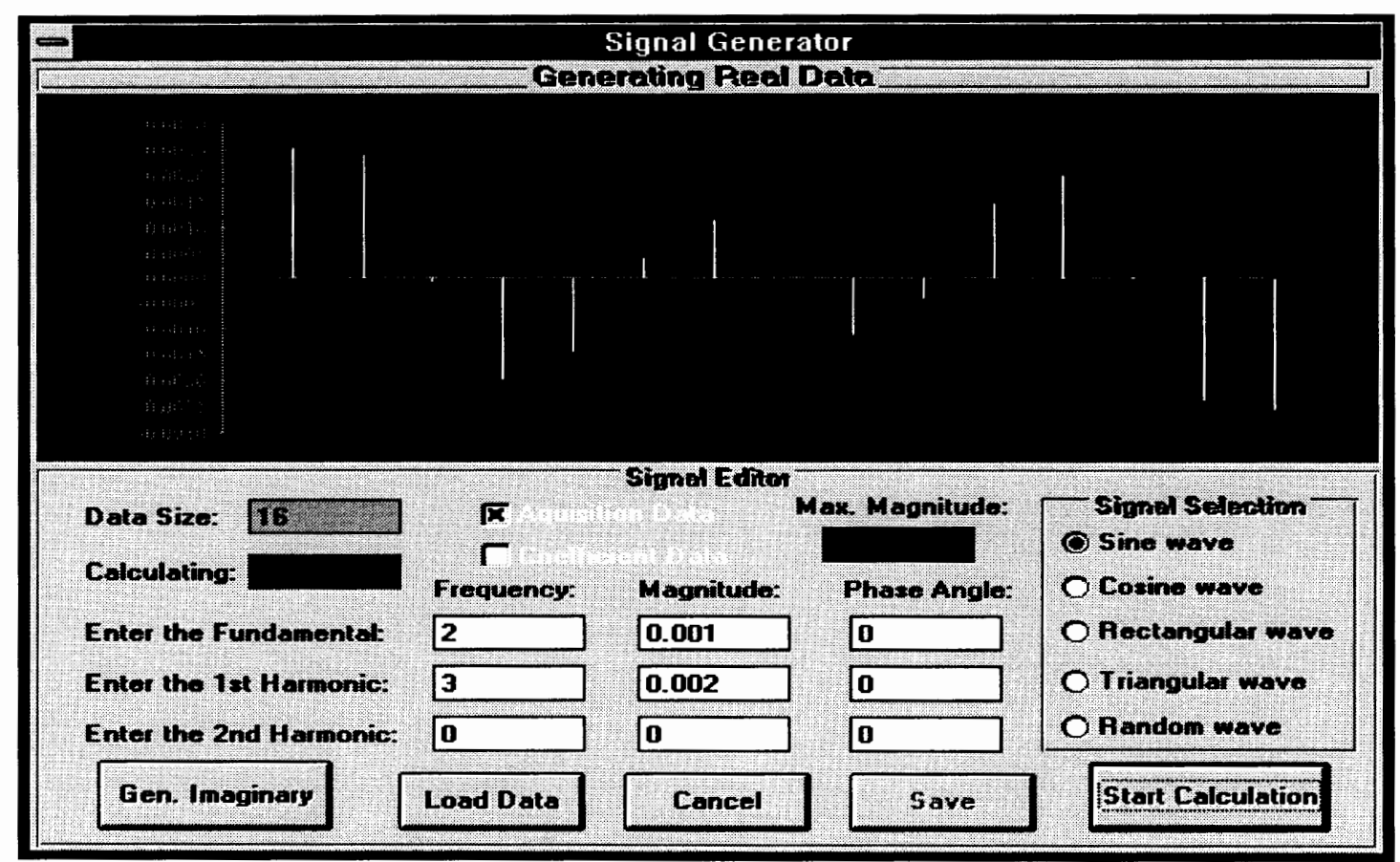

Eigure 22. WinDSP Signal Generator Program Window 
The next step of block level design is to set up the signal configuration of LH9124 and generate the address pattern from LH9320 DOS simulator. By double clicking on the radix-2 icon, a configuration setup window will appeared as shown in Figure 23.

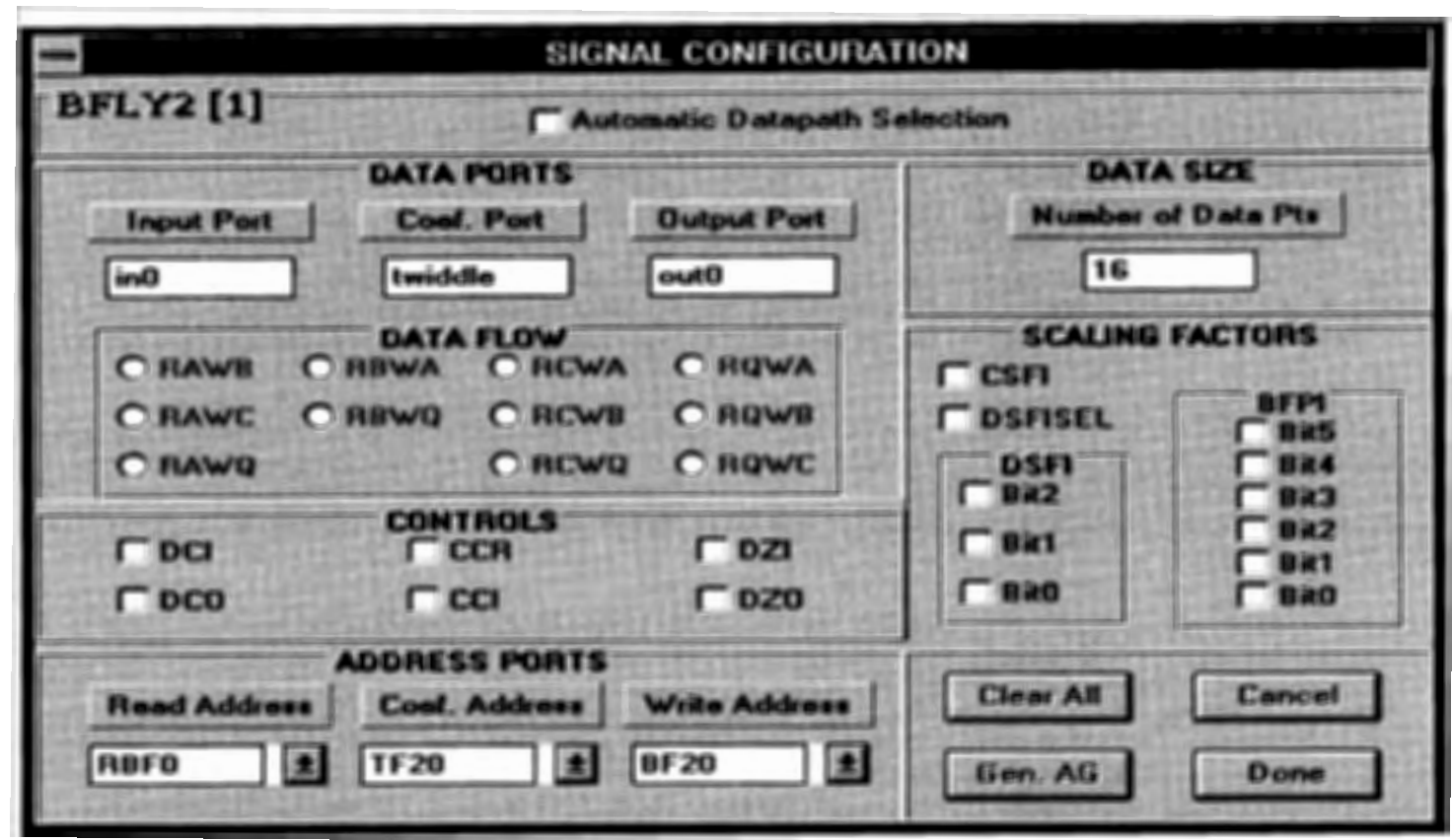

Eigure 23. WinDSP Signal Configuration Window

In Figure 23, the input, coefficient and output port are data files in WinDSP. But in the hardware design, these ports are connected to the physical memory. The address ports consist of the address sequence for the FFT operation. The read address, coefficient address and write address ports are used for reading the input address file, coefficient address file and output address file. This is done according to Table VII and these files are indexing the data for the LH9124 instruction. In this case, its indexed for radix-2 operation. Figure 23 shows the signal configuration setup of the first stage of 16-point radix-2 operation. The signal setup for the subsequent stage will have the same window menu but the data file and the address file will be different. The output data file is always 
used by the input port of the next stage.

Once the signal configuration and parameters are setup, the program is ready for execution. Click on the Start button and the software will compute the example application, in this case the 16-point FFT algorithm using radix-2 butterfly. Finally, the user can examine the computed data by clicking at the Display/Print icon. A window will appear as shown in Figure 24, and display the result (in spectrum graphic) of that stage where display icon is connected. The top menu bar of the window will show to which stage it is connected. The port display window can also display the result in hexadecimal value if the Data button is clicked.

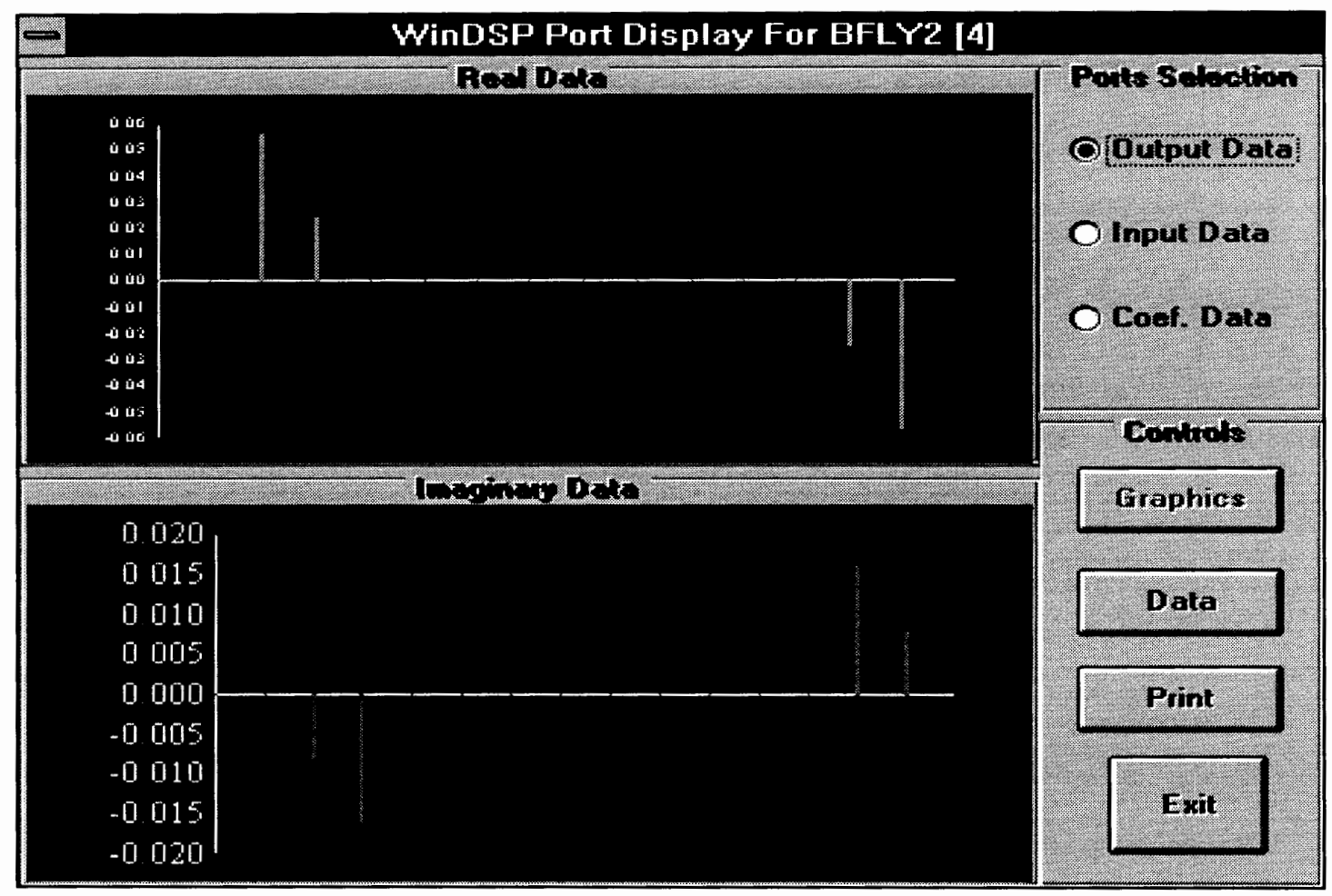

Eigure 24. WinDSP Port Display Window

WinDSP is a very useful tool that has been used by major companies, like G.E. Medical, Catalina Research, Scientific Atlanta, Baka Corporation and Signal Science. 


\section{Example of 2-D FFT Application}

The function block diagram for spectrum sharpening (it is also known as frequency domain filtering) of a $1 \mathrm{~K}$ by $1 \mathrm{~K} 2-\mathrm{D}$ image is shown in the left column of Figure 25. Assume that the row and column FFTs are implemented represented by 4@16@16. The notation “@” represents the cascaded operation. The SFG structure of the 2-D FFT (see Figure 17) is represented by 4@16@16@4@16@16 with 1 Mega elements. It can be seen from Figure 25 that once the function block diagram of the desired application is defined, the required instructions can be directly obtained from the function block diagram to function-level representation of instruction mapping.

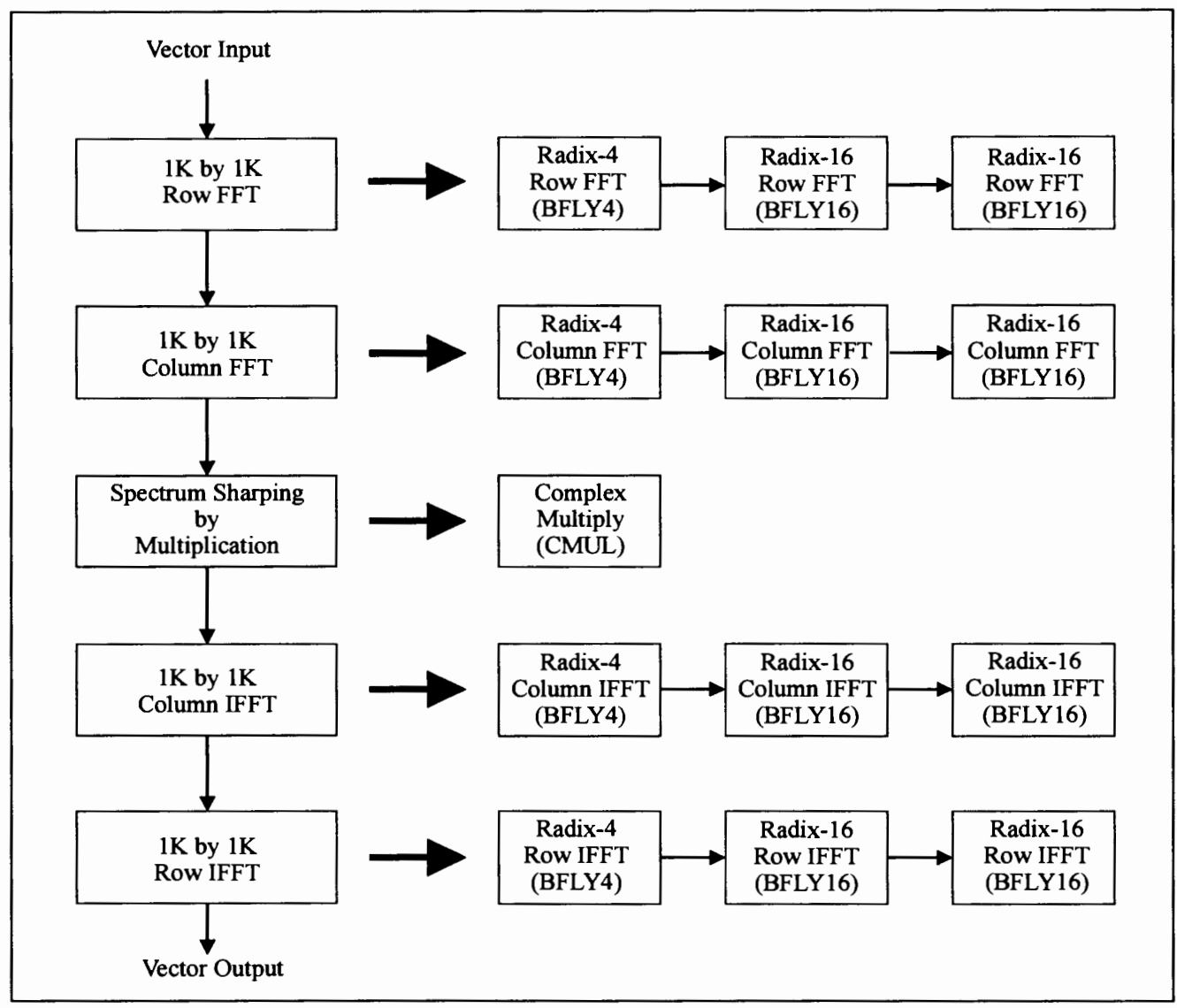

Eigure 25. Block Diagram of $1 \mathrm{~K}$ by $1 \mathrm{~K}$ Image Spectrum Sharpening 
The problem of the 2-D $1 \mathrm{~K}$ by $1 \mathrm{~K}$ image spectrum sharpening can be implemented by the chip set with 13 instructions as listed in Table X. Each function block is executed by one LH9124 instruction and three associated LH9320 instructions to control the input, output and coefficient data transfers. The 13-instruction implementation can be optimized into a 12 instruction implementation because the complex multiply and radix-4 column IFFT stages can be combined into one stage executed by the BWND4 instruction. The BWND4 is one of the LH9124 chip set instructions. It performs both the windowing operation as well as the radix-4 algorithm. Windowing can be considered as a complex multiply operation.

Table X: Spectrum Sharpening of a 1K by 1K 2-D Image

\begin{tabular}{|c|c|c|c|c|}
\hline & LH9124 & \multicolumn{3}{|c|}{ LH9320 } \\
\hline Stage & $\begin{array}{c}\text { Execution } \\
\text { Instructions }\end{array}$ & Inputs & Coefficients & Outputs \\
\hline \hline 1 & BFLY4 & RBF0 & TF40 & BF20 \\
\hline 2 & BFLY16 & BF22 & MXT4160 & BF22 \\
\hline 3 & BFLY16 & BF26 & MXT4161 & BF26 \\
\hline 4 & BFLY4 & BF210 & TF40 & BF210 \\
\hline 5 & BFLY16 & BF212 & MXT4160 & BF212 \\
\hline 6 & BFLY16 & BF216 & MXT4161 & BF216 \\
\hline 7 & CMUL & BF20 & BF20 & BF20 \\
\hline 8 & BFLY4 & RBF0 & TF40 & RBF0 \\
\hline 9 & BFLY16 & BF22 & MXT4160 & BF22 \\
\hline 10 & BFLY16 & BF26 & MXT4161 & BF26 \\
\hline 11 & BFLY4 & BF210 & TF40 & BF210 \\
\hline 12 & BFLY16 & BF212 & MXT4160 & BF212 \\
\hline 13 & BFLY16 & BF216 & MXT4161 & BF216 \\
\hline
\end{tabular}

It should be noted from Figure 25 that the 2-D FFT is implemented in the row- 
column order and the 2-D IFFT in the column-row order. The reason for this is that the row and column indices are swapped when the 2-D bit-reverse addressing is applied. Therefore, if the inputs are in the column-major order, the outputs after the 2-D FFT will be in the row-major order. Similarly, the row-major order inputs will imply the columnmajor order outputs after the 2-D IFFT. Thus, the column-major order inputs imply the column-major order outputs after FFT and IFFT operations. A similar comment can be extended to the M-D case. 


\section{CHAPTER VI}

\section{EVALUATION OF RESULTS AND PERFORMANCE COMPARISON}

The novel FFT algorithms with unified indexing have been implemented in the Sharp LH9124 array processing chip set. The Sharp LH9124 is an execution unit with radix-2, radix- 4 and radix- 16 butterflies built in the highly pipelined data path. The radix2 , radix -4 and radix- 16 butterflies can be implemented within 2,4 , and 16 cycles, respectively. The Sharp LH9320 is a programmable address generator to provide the address patterns required by the LH9124. The unified indexing equations defined in Chapter IV for the input/output data, twiddle factor and bit-reverse sequences are built in the instruction set of the LH9320. Each set of data from the input port to the output port in LH9124 is delayed by a latency of $8,18,20$ or 68 system clocks depending on the function in process as described in Table XI.

Table XI: Latency of the LH9124 Instructions

\begin{tabular}{|l|c|}
\hline \multicolumn{1}{|c|}{ Instructions } & Latency \\
\hline BWND2, BWND4 & 20 cycles \\
\hline BFLY16 & 68 cycles \\
\hline MOVD & 8 cycles \\
\hline All Other Instructions & 18 cycles \\
\hline
\end{tabular}

The number of data to be processed by the FFT can be structured to use different combination of radixes. Table XII shows the example of 1024-point FFT structured in 
four different ways and its performance is compared. It can be seen that the performance is inversely proportional to the number of stages of the structure. The higher the performance, the shorter the stages. Thus, the three stage $4 \times 16 \times 16$ mixed mode structure has the best computing efficiency for the 1024-point FFT. The computing efficiency for the non-mixed mode radix-2 structure is 1 . The computing efficiency of other structures will be compared with that of the radix-2 structure.

Table XII: Performance Comparison of 1024-point FFT Structures

\begin{tabular}{|l|c|c|c|}
\hline Structure & $\mathbf{2 \times 2 \times 2 \times 2 \times 2 \times 2 \times 2 \times 2 \times 2 \times 2}$ & $\mathbf{4 \times 4 \times 4 \times 4 \times 4}$ & $\mathbf{4 \times 1 6 \times 1 6}$ \\
\hline \hline Cycles & 10420 & 5210 & 3276 \\
\hline No. of stages & 10 & 5 & 3 \\
\hline Efficiency & 1 & 2 & 3.18 \\
\hline
\end{tabular}

The execution cycles in each stage depend on the data length only and are independent of the instruction applied. To improve the computing efficiency of the chip set, some functions can be combined together and replaced by one more powerful function. Thus, the speed for the functions can be doubled or quadrupled. For example, one radix- 4 stage can be replaced with two radix- 2 stages and one radix-16 stage can either be replaced with four radix-2 stages or two radix-4 stages. Both the LH9124 and LH9320 provide efficient instructions for these functions. Table XIII lists the instructions to implement the 16-point 1-D FFT and 4 by 4 2-D FFT by the radix-4 (BFLY4) FFT algorithm. The radix-2 algorithm shown in Table VII (on page 73) requires 136 cycles on the LH9124 chip. The radix-4 algorithm shown in Table XIII requires only 68 cycles on the LH9124 chip to finish 16-point FFT for either 1-D or 2-D case. Thus, the computing efficiency improvement is twice by using radix-4 algorithm. 
Table XIII: Two FFT Examples using Radix-4 with LH9124/LH9320 Instructions

\begin{tabular}{|c|c|c|c|c|c|c|c|c|}
\hline & \multicolumn{3}{|c|}{ 16-point 1-D FFT } & \multicolumn{3}{c|}{ 4 by 4 2-D FFT } \\
\hline Stage & Function & Input & Twiddle & Output & Function & Input & Twiddle & Output \\
\hline 1 & BFLY4 & RBF0 & TF40 & BF40 & BFLY4 & RBF0 & TF40 & BF40 \\
\hline 2 & BFLY4 & BF41 & TF41 & BF41 & BFLY4 & BF41 & TF40 & BF41 \\
\hline
\end{tabular}

Table XIV compares the performance of the 64K-point 1-D FFT with 256 by 256 2-D FFT. It can be seen that both have the same performance because the data block size, the number of instructions, and the instruction pipeline overhead are all the same. It should be noted by using radix-2 instruction for the 2-D FFT, only 16 instructions (or stages) are required for the unified 2-D FFT implementation instead of 4096 instructions required by the traditional 2-D FFT implementation. Therefore, the instruction pipeline latency can be greatly reduced. With 25 nanoseconds machine cycle time, the 256 by 256 2-D complex FFT using radix-16 can be finished with 6.56 milliseconds.

Table XIV: Benchmark of Unified Indexing FFT Algorithms of Different Radix

\begin{tabular}{|c|c|c|c|c|c|c|}
\hline & \multicolumn{3}{|c|}{ 64K-point 1-D FFT } & \multicolumn{3}{c|}{ 256 by 256 2-D FFT } \\
\hline Butterfly & stages & cycles & msec & stages & cycles & msecs \\
\hline \hline Radix-2 & 16 & 1048864 & 26.22 & 16 & 1048864 & 26.22 \\
\hline Radix-4 & 8 & 524432 & 13.11 & 8 & 524432 & 13.11 \\
\hline Radix-16 & 4 & 262416 & 6.56 & 4 & 262416 & 6.56 \\
\hline
\end{tabular}

\section{Computational Complexity Model}

The efficiency of the unified FFT algorithm is most often measured by the rate of growth of time and hardware required to solve problems of larger and larger size. In association with each instance of problem is a number $n$, in this case, the number of data to be processed. The other related measure is the number of computational stages. 
Therefore, the total number of machine cycles for an FFT implementation is calculated as

$$
\text { Cycles }=\sum_{i=1}^{s}\left(N_{i}+P O_{i}\right)
$$

where $N_{i}$ and $P O_{i}$ denote the data block size and the pipelined overhead of the i-th instruction, respectively. $s$ is the number of instructions or butterfly stages. Equation (6.1) models the unified indexing FFT algorithm complexity of LH9124/LH9320 chip set. It can be used for non-mixed and mixed radix mode structure. The number of cycles computed by Equation (6.1) is also the actual machine cycle for the hardware chip set. In the real world applications of the LH9124/LH9320 chip set, the external hardware parameters can affect the performance of the unified FFT algorithm. These parameters can be: slow I/O bus speed, slow memory speed, and other delays. The parameters can also be considered as overheads to the instructions and should be included with the calculation of the machine cycles.

Table XV on page 86 shows the benchmark of the unified FFT alogrithm based on radix-2 instruction. The LH9124 DSP processor is running at $40 \mathrm{MHz}$ clock speed. Calculation of the computation time (in msecs) in Table XV is derived by

$$
\text { Computation time }(\text { in msecs })=(\text { Cycles } * \text { Clock Speed })
$$

The number of FFT used for the specific data size is computed as such:

$$
\text { No. of FFT per seconds }=(\text { Cycles } / \text { Clock speed })
$$

In the real world, the number of data used the FFT algorithms applications are at least 256 and at most $2 \mathrm{M}$ bytes. 
Table XV: Benchmark of Unified Indexing Algorithm using Radix-2

\begin{tabular}{|c|c|c|c|c|}
\hline \multicolumn{5}{|c|}{ Unified (1-D) FFT Algorithm using Radix-2 only } \\
\hline Data Size & Stages & Cycles & msecs & FFT/second \\
\hline \hline 256 & 8 & 2192 & 0.0548 & $8.77 \mathrm{e}+10$ \\
\hline 512 & 9 & 4770 & 0.1192 & $1.91 \mathrm{e}+11$ \\
\hline 1024 & 10 & 10420 & 0.2605 & $4.17 \mathrm{e}+11$ \\
\hline 2048 & 11 & 22726 & 0.5681 & $9.09 \mathrm{e}+11$ \\
\hline 4096 & 12 & 49368 & 1.2342 & $1.97 \mathrm{e}+12$ \\
\hline 8192 & 13 & 106730 & 2.6682 & $4.27 \mathrm{e}+12$ \\
\hline 16384 & 14 & 229628 & 5.7407 & $9.19 \mathrm{e}+12$ \\
\hline 32768 & 15 & 491790 & 12.295 & $1.97 \mathrm{e}+13$ \\
\hline 65536 & 16 & 1048864 & 26.222 & $4.20 \mathrm{e}+13$ \\
\hline 131072 & 17 & 2228530 & 55.713 & $8.91 \mathrm{e}+13$ \\
\hline 262144 & 18 & 4718916 & 117.97 & $1.89 \mathrm{e}+14$ \\
\hline 524288 & 19 & 9961814 & 249.04 & $3.98 \mathrm{e}+14$ \\
\hline 1048576 & 20 & 20971880 & 524.29 & $8.39 \mathrm{e}+14$ \\
\hline 2097152 & 21 & 44040570 & 1101.0 & $1.76 \mathrm{e}+15$ \\
\hline
\end{tabular}

Three sets of graphs (see pages 87 and 88 ) have been plotted from Table XV. Figure 26 shows the graph of number of cycles versus the number of data. Figure 27 shows computation time in milliseconds versus the data size and Figure 28 shows the number of FFT per second against the data size. The shapes of all three graphs are proportional to $\mathrm{N} \log _{2} \mathrm{~N}$. From the three graphs, the unified indexing FFT algorithm satisfied Knuth's definition [Knuth, 1981] for Omicron $(O)$ asymptotic order. Thus, the unified indexing algorithm is an $O\left(N \log _{2} N\right)$ complex FFT computation. It should be noted that the larger the data size (i.e the number of data), the less significant the pipeline latency. Moreover, the external hardware overheads are also becoming negligible. The 
data for the LH9124 instructions are usually implemented in block fashion manner. The computation overheads occur when data transfers from one block to other block, but not from one element of data to the other. In order to reduce the proportion of overhead in computations, the length of the block data should be as large as possible. The 2-D to 1-D FFT mapping has implicitly included the concept of extending the block length because the length of the FFT is the whole 2-D array of elements instead of one row or one column of elements.

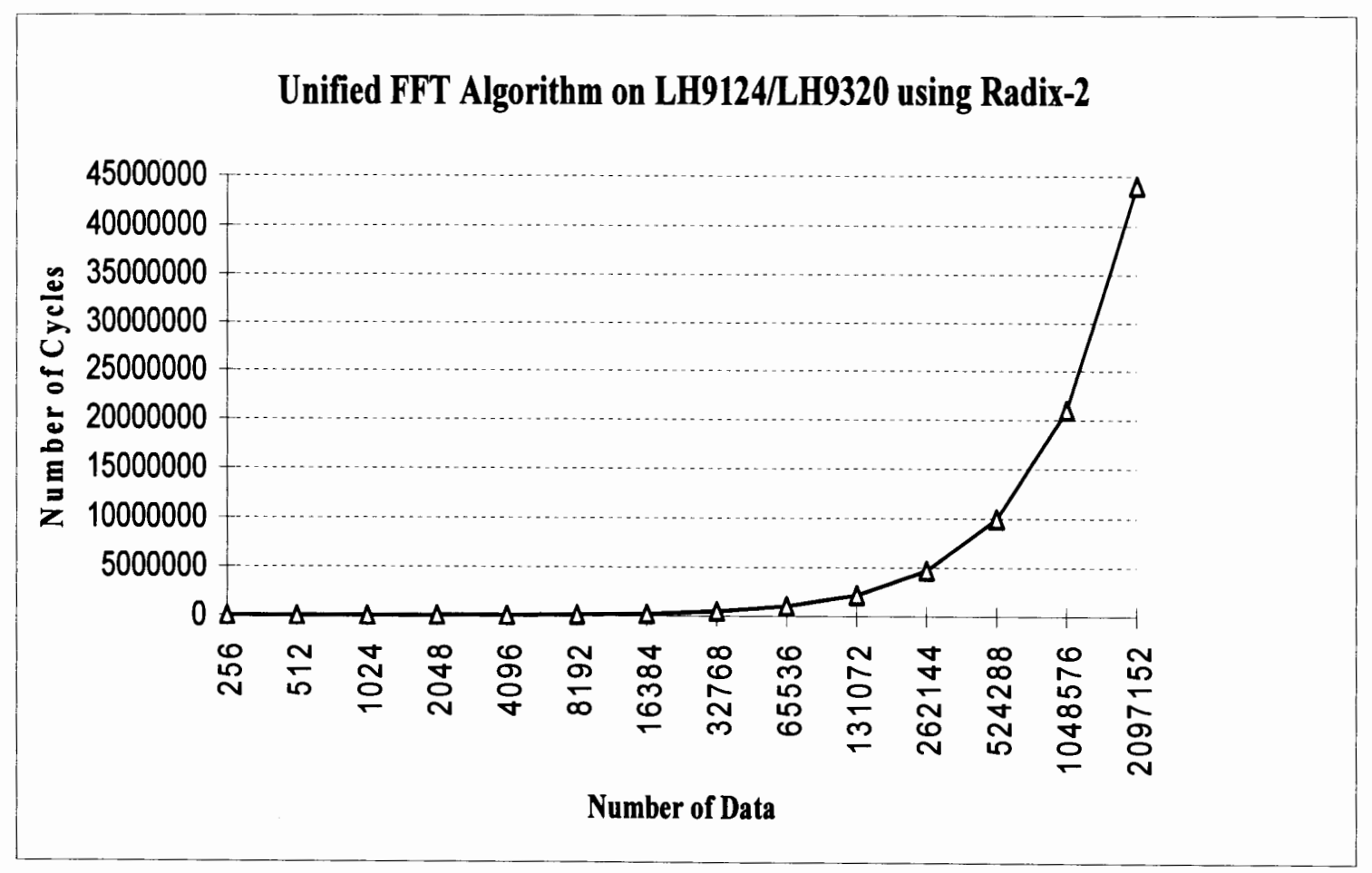

Eigure 26. Graph of Actual Computation Cycles vs. Data Size on LH9124/LH9320 


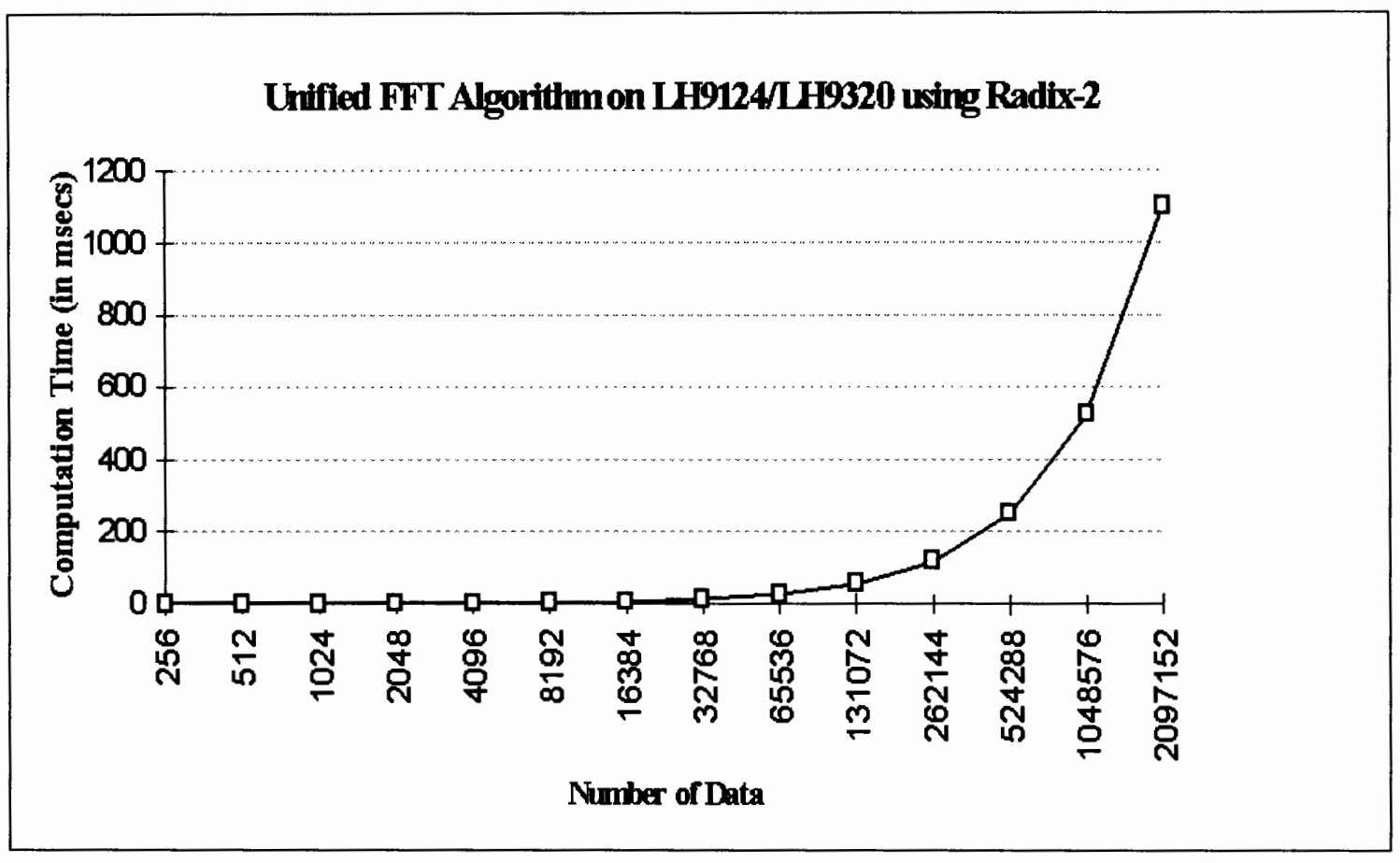

Eigure 27 Graph of Actual Computation Time vs. Data Size on LH9124/LH9320

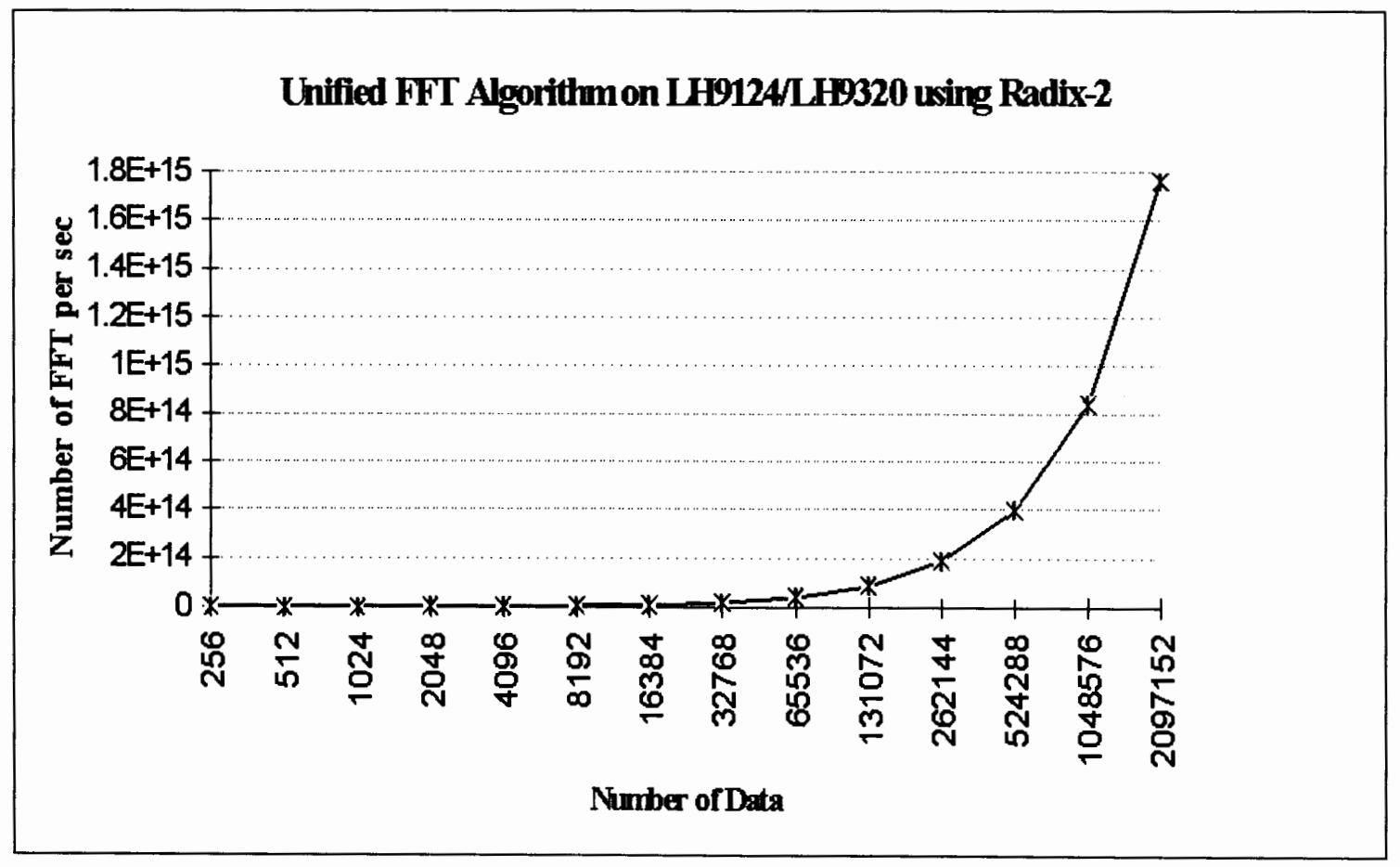

Eigure 28. Graph of Number of FFT per second vs. Data Size on LH9124/LH9320 


\section{Multi-Dimension FFT Applications}

There are two meanings for the instructions optimized in the functionrepresentation level. For chip designers, each function instruction is optimized in a parallel and pipelined form and built in the data path. For users, the optimum performance for their applications is to select one implementation that requires the minimum number of stages.

Table XVI: Benchmark of M-D FFT Applications on LH9124/LH9320

\begin{tabular}{|l|c|c|c|}
\hline $\begin{array}{l}\text { FFT Applications } \\
\text { (Based on 25nsec per cycle) }\end{array}$ & Stages & $\begin{array}{c}\text { Execution } \\
\text { Cycles }\end{array}$ & $\begin{array}{c}\text { Time } \\
\text { (msec) }\end{array}$ \\
\hline 64K-Point 1-D FFT & 4 & 262416 & 6.56 \\
\hline 256 by 256 2-D FFT (Unified) & 4 & 262416 & 6.56 \\
\hline 256 by 256 2-D FFT (Traditional) & $(1024+256)^{*}$ & $401920^{*}$ & 10.05 \\
\hline 4K-Point 1-D FFT & 3 & 12492 & 0.312 \\
\hline 64 by 64 by 64 3-D FFT (Unified) & 6 & 1573122 & 39.328 \\
\hline 64 by 64 by 64 3-D FFT (Traditional) & 24576 & 2629632 & 65.741 \\
\hline 1K by 64-Channel Array Beamforming (U) & 7 & 265542 & 6.639 \\
\hline 1K by 1K 2-D Spectrum Filtering (U) & 12 & 12583530 & 314.59 \\
\hline 256 by 64-Channel DFT Filter Bank (U) & 7 & 115064 & 2.877 \\
\hline * denotes there is an extra term added to the calculation. (U) denotes Unified \\
\hline
\end{tabular}

Table XVI shows the benchmarking of M-D FFT applications on LH9124/ LH9320 chip set. The computing time of the FFT depends on the total number of data in the array, and is independent on the dimension of FFT. For example, both the 256 by 256 points two-dimension (2-D) FFT and the 64K-point one-dimension (1-D) FFT can use the same 1-D indexing, and execute very fast within the execution unit. The traditional way of computing the 256 by 256 points two-dimensional (2-D) FFT is procedures intensive. 
For example, to be consistent with the benchmark shown in Table XVI, first, compute each row of vectors matrix with two radix-16. There are 256 rows to compute. Second, search the maximum block-floating point scale factor on each row for 256 times. Then each of the 256 rows is scaled with the maximum scaling factor to prevent overflow. Repeat the steps for the column vectors matrix. Next, do a transposition on the scaled vectors from the row side. Finally, multiply the transposed vectors with the scaled vectors from the column side. Thus, the final result is the matrix vectors in frequency domain after the FFT. As it can be seen, there are many steps which the traditional 2-D FFTs have to go through. The overall overheads from each of these prodecures can be, therefore, significant as compared with the unified indexing method. Thus, to compute the number of machine cycles for 256 by 256 2-D FFT in a traditional method, the formula is used:

$$
\text { Cycles }=\sum_{i=1}^{s} 2\left(N_{i}+P O_{i}\right)+\sum_{i=1}^{s}\left(T_{i}+P O_{i}\right)+k_{i}
$$

where $N_{i}$ and $P O_{i}$ denote the data block size for row/column operation and the pipelined overhead of the $\mathrm{i}$-th instruction, respectively. $T_{i}$ denotes the data block size for transposition operation. $s$ is the number of instructions or butterfly stages. $k$ is the number of cycles required for searching the block-floating scaling factor.

The unified indexing of FFT algorithm has provided significant improvement to 2D FFT computation, as well as to the performance of the system. 


\section{CHAPTER VII}

\section{CONCLUSIONS AND FUTURE WORK}

From the vector-matrix representation of the FFT algorithms, this thesis shows that the unified indexing methodology for the 2-D to 1-D FFTs has provided significant improvements to the performance of 2-D FFT architectures. The unified indexing approach, essentially, can be extended to more general multidimensional FFTs in a straightforward manner. The development of the WinDSP simulator tool with unified indexing helps the users to evaluate and design the multi-dimension FFT applications as 1-D FFT applications. To illustrate the simplest implementation for the unified indexing method, this thesis presented examples of 16-point 1-D FFT and 4 by 42 -D FFT. The equivalent relationships between the 1-D FFT and M-D FFT were defined, and the mappings of function-level representation of instructions to the unified indexing structure were explained and illustrated. From Table XVI, the unified method used less instructions (stages) and obtained better performance than the traditional method.

There are a lot of advantages of using the Unified Indexing Approach [Ju, 1993]. First, only 1-D indexing is required for all the M-D FFT implementations. Second, the data matrix transpose is not required for the M-D FFT. Third, the definition and design of the address generator can be simplified. Fourth, the scaling problems of block floatingpoint arithmetic are automatically solved. Fifth, the program length can be greatly reduced. Finally, the unified indexing concept can be extended to solve scaling problems 
of block floating-point arithmetic for the general DSP applications.

The FFT algorithm is computing-intensive and its architecture has some form of regularity. Therefore, it is a good candidate to be implemented by cascaded or parallel architectures. The LH9124 is suitable for cascaded or parallel architecture because of its by-pass form structure. The four data ports of the LH9124 have fast data transfer rates. The data from these ports do not go through holding registers. Thus, the throughput is improved by the cascaded architecture, and the latency by the parallel architecture. This implementation of cascaded and parallel architectures can be the future work.

The decomposition process of the DFT shows that the FFT inherently has the cascaded form. Each stage of the FFT can be implemented by one radix instruction of the LH9124. Hence, the N-stage FFT can be implemented by N cascaded LH9124 processors. The block output data of the i-th stage will be the block input data of the $(\mathrm{i}+1)$-th stage. The computation of the $(\mathrm{i}+1)$-th stage will wait for the completion of the i-th stage because of data dependency.

The alternative way to improve the throughput for the FFT is obtained by parallel processing. The advantage of the parallel architecture is that each processor can run an independent job or algorithm. For an N-point FFT with M stages, the time required for a single LH9124 to finish the operation is about $\mathrm{N}^{*} \mathrm{M}$ cycles. Thus, by multiplexing, the $\mathrm{N}^{*} \mathrm{M}$ points of data can be loaded to $\mathrm{M}$ processors with each processor having $\mathrm{N}$ points.

The unified indexing method can also be extended to many other block transforms algorithm. Examples of these transforms are fast cosine transform, Reed-Muller, Walsh and etc. 


\section{REFERENCES}

M. Burnett, A. Goldberg, and T. Lewis, eds., "Visual Object-Oriented Programming: Concepts and Environments", Prentice-Hall, New York, 1995.

W.H. Chen and C.H. Smith, "A Fast Computation Algorithm for the Discrete Cosine Transform," IEEE Trans. Comm., vol. COM-25, no. 9, pp. 10041009, Sep. 1977.

J.W. Cooley and J.W. Tukey, "An Algorithm for the Machine Calculation of Complex Fourier Series," Math. of Comput., vol. 19, pp. 297-301, 1965.

T. Crystal and L. Kulsrud, "Circus," CRD Working Paper, Inst. Def. Analysis, Princeton, NJ, Dec. 1974.

M. Dertouzous, M. Kaliske and K. Polzen, "On line simulation of block-diagram systems," IEEE Trans. Comput., Vol. C-18, Apr. 1969.

D.E. Dudgeon and R.M. Mersereau, "Multidimensional Digital Signal Processing," Prentice-Hall, Englewood Cliffs, New Jersey, 1984.

D.F. Elliot and K.R. Rao, "Fast Transform, Algorithms, Analyses, Applications," Academic Press, 1982.

E.P. Glinert and S. L. Tanimoto, "Pict: An interactive graphical programming environment," Computer, vol. 17, 1984.

B. Gold and C. Rader, "Digital Processing of Signals," McGraw-Hill, New York, 1969.

S. Haykin, ed., “Array Signal Processing,” Englewood Cliffs, New Jersey, 1985.

D. D. Hils, "Visual Languages and Computing Survey: Data Flow Visual Programming Languages," J. Visual Languages and Computing, Vol. 3, No. 1, pp. 69-101, 1992.

W. H. Ho, E. A. Lee and d. G. Messerschmitt, "High level data flow programming for digital signal processing," presented at the VLSI DSP Workshop, IEEE ASSP Soc., Monterey, CA, Nov 1988. 
K. Hwang and F.A. Briggs, "Computer Architecture and Parallel Processing," McGraw-Hill, New York, 1984.

Hypersignal-Window Analysis/Design Software, Hyperception Inc, Dallas, Texas.

D. H. Johnson and R. E. Vaughan, "A Software environment for digital signal processing simulations," Circuits, Syst., Signal Processing, vol. 6, pp. 3144, Jan 1987.

R. Jordan and The Khoros Group, "Khoros: A Software Development Environment for Data Processing," DSP applications, pp 16-23, March 1993.

C-J. Ju and M. Fleming, "Design concept of real-time array signal processors." Proceeding of the International Conference on Signal Processing Applications and Technology, Boston, pp. 188-197, Nov 1992.

C-J. Ju, "Algorithms of defining 1-D indexing for M-D mixed radix FFT implementation," Proceeding of IEEE Pacific Rim Conference on Communications, Computers and Signal Processing, Victoria, Canada, May 1993.

C-J. Ju and W. Alexander, "Block Realization of Multidimensional IIR Digital Filters and its Finite Word Effects," IEEE Trans. on Circuits and Systems, pp. 1030-1044, Sept. 1987.

D.E. Knuth, "The Art of Computer Programming, Seminumerical Algorithms," 2nd ed., Addison-Wesley Publishing Co., Reading, MA, 1981.

G. Korn, "High-speed block-diagram languages for microprocessors and mincomputers in instrumentation, control and simulation," Comput. Elec. Eng., vol. 4, pp. 143-159, 1977.

S.Y. Kung, "VLSI Array Processors," Prentice Hall, Englewood Cliffs, New Jersey, 1988.

E.A. Lee, W.H. Ho, E.E. Goei, J.C. Bier and S. Bhattacharyya, "Gabriel: A Design Environment for DSP," IEEE Trans. on ASSP, vol. 37, no. 11, pp. 1751-1762, Nov 1989.

E.A. Lee, and D.G. Messerschmitt, "Static scheduling of synchronous data flow programs for digital signal processing," IEEE Trans. Comput., vol. C-36, Jan 1987.

LH9124 Digital Signal Processor User's Guide, Sharp Electronics Corp, 1992. 
LH9320 Address Generator User's Guide, Sharp Electronics Corp, 1992.

J. Makhoul, "A Fast Cosine Transform in One and Two Dimension," IEEE Trans Acoust., Speech and Signal Proc. vol. ASSP-28, no. 1, pp. 27-34, Feb 1980 .

C. Myers, "Signal representation for symbolic and numerical processing," Ph.D. dissertation, Res. Lab. Electron., Cambridge, MA, M.I.T. Tech. Rep. 521, Aug. 1986.

K.N. Ngan, "Image Display Techniques Using the Cosine Transform," IEEE Trans. Acoust., Speech and Signal Processing, vol. ASSP-32, no. 1, pp. 173-177, Feb 1984.

A.V. Oppenheim and R.W. Schafer, "Discrete-Time Signal Processing," Prentice-Hall, Englewood Cliffs, New Jersey, 1989.

F. Penz, "Visual Programming in the Object World," J. Visual Languages and Computing, Vol. 2, No. 1, pp. 17-41. 1991.

W.K. Pratt, “Digital Image Processing,” John Wiley \& Sons, New York, 1978.

J. Rasure, C. Williams, "An Integrated Data Flow Visual Language and Software Development Environment," Journal of Visual Languages and Computing, pp 217-246, Feb 1991.

J. Rasure, D. Koechner, R. Jordan, C. Williams, "A Design Environment for Multidimensional Signal Processing," Informatica y Automatica Journal, Vol 24, Number 1, pp. 18-23. 1992.

R.C. Reininger and J.D. Gibson, "Distribution of the Two-Dimensional DCT Coefficients for Images," IEEE Trans. Comm. vol. COM-31, no. 6, pp. 835-839, June 1983.

K. S. Shanmugan, G. J. Minden, E. Komp, T. C. Manning, and E. R. Wiswell, "Block-oriented system simulator (BOSS)," Telecommunication Lab., University of Kansas, Int. Memo., 1987.

N. C. Shu, "Visual Programming,” Van Nostrand Reinhold, 1988.

M. A. Zissman, G. C. O'Leary and D. H. Johnson, "A block diagram compiler for a digital signal processing MIMD computer," DSP Workshop Presentation, Chatham, MA, Oct. 1986. 


\section{APPENDIX A}

\section{ANALYSIS OF UNIFIED INDEXING FOR 1-D TO M-D FFT}

In chapter IV, a novel vector-matrix form to represent the unified indexing of FFT algorithm and the relationship of 1-D and M-D FFT were presented. The implementation structures for both the Bit-reverse-Input/Linear-Output (BI/LO) and Linear-Input/Bitreverse-Output (LI/BO) for computing the FFT were be shown. In this appendix, a vector matrix representation of 16-point FFT is used as the example. The example here is the analysis and expansion of the Bit-reverse Input and Linear Output (BI/LO) 16-point 1-D FFT using unified indexing definition. Appendix B will illustrate the expansion for the BI/LO 4 by 4 2-D FFT. The results of the two examples will be listed in Table XVII and compared with the address generated by Sharp LH9320 chip set. The implementation method for the LI/BO structure is similar to the one from the examples below.

The vector-matrix form of the 1-D BI/LO FFT with the length $\mathrm{N}=2^{\mathrm{s}}$ can be obtained as follows:

$$
\underline{X}=F G_{s}(B I(s)) \times F G_{s-1}(B I(s-1)) \times \ldots \times F G_{1}(B I(1)) \times P_{b r} \times \underline{x}
$$

The $k$-th butterfly stage of the BI/LO FFT can be further decomposed into three cascaded matrices:

$$
F G_{k}(B I(k))=P_{l k} \times B I(k) \times P_{r k}
$$

where 


$$
P_{r k}(n, m)= \begin{cases}1 & \text { for } n=\frac{N}{2^{s+1-k}} \times \operatorname{Mod}(m)_{2^{s+1-k}}+\operatorname{Int}\left(\frac{m}{2^{s+1-k}}\right) \\ 0 & \text { otherwise }\end{cases}
$$

and

$$
P_{l k}(n, m)= \begin{cases}1 & \text { for } m=\frac{N}{2^{s+1-k}} \times \operatorname{Mod}(n)_{2^{s+l-k}}+\operatorname{Int}\left(\frac{n}{2^{s+1-k}}\right) \\ 0 & \text { otherwise }\end{cases}
$$

The bit-reverse matrix is as follows:

$$
P_{b k}(n, m)= \begin{cases}1 & \text { for } n=b r(m) \\ 0 & \text { otherwise }\end{cases}
$$

where $\operatorname{br}(m)$ denotes the bit-reverse operation on the index $m$ with the number of bits $s$ as follows:

$$
\operatorname{br}(m)_{s}=\operatorname{br}\left(m_{s-1} m_{s-2} \ldots m_{l} m_{0}\right)_{s}=m_{0} m_{1} \ldots m_{s-2} m_{s-1}
$$

The butterfly operation matrix for the BI/LO FFT is defined as

$$
B I(k)=\left[\begin{array}{cccc}
b i_{k}(0) & 0 & \ldots & 0 \\
0 & b i_{k}(1) & \ldots & 0 \\
\ldots & \ldots & \ldots & \ldots \\
0 & 0 & \ldots b i_{k}(N / 2-1)
\end{array}\right]
$$

The radix- 2 butterfly module $b i_{k}(n)$ of located the diagonal of $B I(k)$ is defined as

$$
b i_{k}(n)=\left[\begin{array}{cc}
W_{N}^{0} & W_{N}^{i} \\
W_{N}^{0} & -W_{N}^{i}
\end{array}\right] \quad \text { with } i=\frac{N}{2^{k}} \times \operatorname{Int}\left(\frac{n}{2^{s-k}}\right)
$$




\section{Computation of 1-D BI/LO 16-point FFT Vector Matrix}

To compute the Bit-reverse Input and Linear Output of 16-point 1-D FFT, substitute Equation (A.2) into Equation (A.1) and expand the permutation matrix of the left and the right for each individual stage.

$$
\begin{aligned}
\underline{X}= & P_{l 4} \times B I(4) \times P_{r 4} \times P_{l 3} \times B I(3) \times P_{r 3} \times P_{l 2} \\
& \times B I(2) \times P_{r 2} \times P_{l 1} \times B I(1) \times P_{r 1} \times P_{b r} \times \underline{x}
\end{aligned}
$$

where $P_{b r}$ and $x$ are bit-reverse permutation matrix and input vector, respectively.

As you can see from Equation (A.9), the input vectors to be computed are not arranged as bit-reverse sequence. Therefore the bit-reverse permutation matrix is required to do the FFT. The computation process for the BI/LO 16-point 1-D FFT starts from the right and is propagated to the left of Equation (A.9). The expansion of the bit-reverse permutation matrix is as follows:

$$
P_{b r}=\left[\begin{array}{llllllllllllllll}
1 & 0 & 0 & 0 & 0 & 0 & 0 & 0 & 0 & 0 & 0 & 0 & 0 & 0 & 0 & 0 \\
0 & 0 & 0 & 0 & 0 & 0 & 0 & 0 & 1 & 0 & 0 & 0 & 0 & 0 & 0 & 0 \\
0 & 0 & 0 & 0 & 1 & 0 & 0 & 0 & 0 & 0 & 0 & 0 & 0 & 0 & 0 & 0 \\
0 & 0 & 0 & 0 & 0 & 0 & 0 & 0 & 0 & 0 & 0 & 0 & 1 & 0 & 0 & 0 \\
0 & 0 & 1 & 0 & 0 & 0 & 0 & 0 & 0 & 0 & 0 & 0 & 0 & 0 & 0 & 0 \\
0 & 0 & 0 & 0 & 0 & 0 & 0 & 0 & 0 & 0 & 1 & 0 & 0 & 0 & 0 & 0 \\
0 & 0 & 0 & 0 & 0 & 0 & 1 & 0 & 0 & 0 & 0 & 0 & 0 & 0 & 0 & 0 \\
0 & 0 & 0 & 0 & 0 & 0 & 0 & 0 & 0 & 0 & 0 & 0 & 0 & 0 & 1 & 0 \\
0 & 1 & 0 & 0 & 0 & 0 & 0 & 0 & 0 & 0 & 0 & 0 & 0 & 0 & 0 & 0 \\
0 & 0 & 0 & 0 & 0 & 0 & 0 & 0 & 0 & 1 & 0 & 0 & 0 & 0 & 0 & 0 \\
0 & 0 & 0 & 0 & 0 & 1 & 0 & 0 & 0 & 0 & 0 & 0 & 0 & 0 & 0 & 0 \\
0 & 0 & 0 & 0 & 0 & 0 & 0 & 0 & 0 & 0 & 0 & 0 & 0 & 1 & 0 & 0 \\
0 & 0 & 0 & 1 & 0 & 0 & 0 & 0 & 0 & 0 & 0 & 0 & 0 & 0 & 0 & 0 \\
0 & 0 & 0 & 0 & 0 & 0 & 0 & 0 & 0 & 0 & 0 & 1 & 0 & 0 & 0 & 0 \\
0 & 0 & 0 & 0 & 0 & 0 & 0 & 1 & 0 & 0 & 0 & 0 & 0 & 0 & 0 & 0 \\
0 & 0 & 0 & 0 & 0 & 0 & 0 & 0 & 0 & 0 & 0 & 0 & 0 & 0 & 0 & 1
\end{array}\right]
$$




$$
\text { For Stage } 1=P_{l 1} * B I(1) * P_{r l} \text { (i.e. set } \mathrm{k}=1, \mathrm{~s}=4, \mathrm{~N}=16 \text { ) }
$$

$F G_{1}(B I(1))=\left[\begin{array}{llllllllllllllll}1 & 0 & 0 & 0 & 0 & 0 & 0 & 0 & 0 & 0 & 0 & 0 & 0 & 0 & 0 & 0 \\ 0 & 1 & 0 & 0 & 0 & 0 & 0 & 0 & 0 & 0 & 0 & 0 & 0 & 0 & 0 & 0 \\ 0 & 0 & 1 & 0 & 0 & 0 & 0 & 0 & 0 & 0 & 0 & 0 & 0 & 0 & 0 & 0 \\ 0 & 0 & 0 & 1 & 0 & 0 & 0 & 0 & 0 & 0 & 0 & 0 & 0 & 0 & 0 & 0 \\ 0 & 0 & 0 & 0 & 1 & 0 & 0 & 0 & 0 & 0 & 0 & 0 & 0 & 0 & 0 & 0 \\ 0 & 0 & 0 & 0 & 0 & 1 & 0 & 0 & 0 & 0 & 0 & 0 & 0 & 0 & 0 & 0 \\ 0 & 0 & 0 & 0 & 0 & 0 & 1 & 0 & 0 & 0 & 0 & 0 & 0 & 0 & 0 & 0 \\ 0 & 0 & 0 & 0 & 0 & 0 & 0 & 1 & 0 & 0 & 0 & 0 & 0 & 0 & 0 & 0 \\ 0 & 0 & 0 & 0 & 0 & 0 & 0 & 0 & 1 & 0 & 0 & 0 & 0 & 0 & 0 & 0 \\ 0 & 0 & 0 & 0 & 0 & 0 & 0 & 0 & 0 & 1 & 0 & 0 & 0 & 0 & 0 & 0 \\ 0 & 0 & 0 & 0 & 0 & 0 & 0 & 0 & 0 & 0 & 1 & 0 & 0 & 0 & 0 & 0 \\ 0 & 0 & 0 & 0 & 0 & 0 & 0 & 0 & 0 & 0 & 0 & 1 & 0 & 0 & 0 & 0 \\ 0 & 0 & 0 & 0 & 0 & 0 & 0 & 0 & 0 & 0 & 0 & 0 & 1 & 0 & 0 & 0 \\ 0 & 0 & 0 & 0 & 0 & 0 & 0 & 0 & 0 & 0 & 0 & 0 & 0 & 1 & 0 & 0 \\ 0 & 0 & 0 & 0 & 0 & 0 & 0 & 0 & 0 & 0 & 0 & 0 & 0 & 0 & 1 & 0 \\ 0 & 0 & 0 & 0 & 0 & 0 & 0 & 0 & 0 & 0 & 0 & 0 & 0 & 0 & 0 & 1\end{array}\right]$

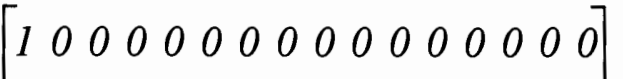

$$
\begin{aligned}
& 0100000000000000 \\
& 0010000000000000
\end{aligned}
$$

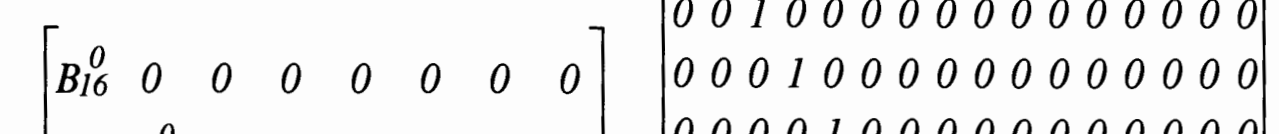

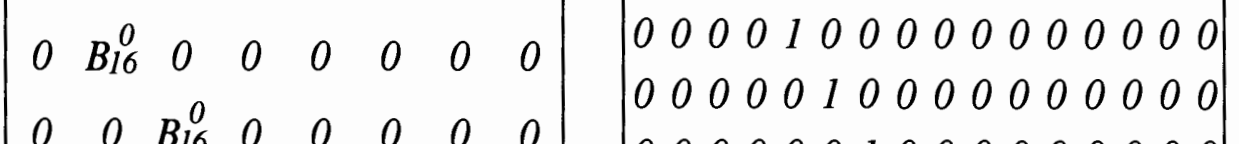

$$
\begin{aligned}
& \times\left[\begin{array}{cccccccc}
0 & 0 & B_{16} & 0 & 0 & 0 & 0 & 0 \\
0 & 0 & 0 & B_{16}^{0} & 0 & 0 & 0 & 0 \\
0 & 0 & 0 & 0 & B_{16} & 0 & 0 & 0
\end{array} \mid \times\left(\begin{array}{cccccccccccccccc}
0 & 0 & 0 & 0 & 0 & 0 & 1 & 0 & 0 & 0 & 0 & 0 & 0 & 0 & 0 & 0 \\
0 & 0 & 0 & 0 & 0 & 0 & 0 & 1 & 0 & 0 & 0 & 0 & 0 & 0 & 0 & 0 \\
0 & 0 & 0 & 0 & 0 & 0 & 0 & 0 & 1 & 0 & 0 & 0 & 0 & 0 & 0 & 0
\end{array} \mid\right.\right.
\end{aligned}
$$

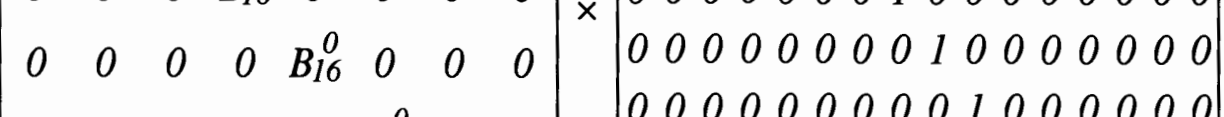

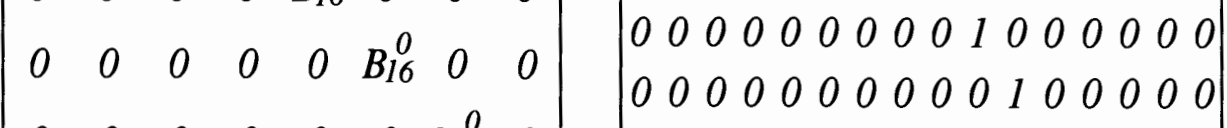

$$
\begin{aligned}
& \begin{array}{ccccccccc}
0 & 0 & 0 & 0 & 0 & 0 & B_{16}^{0} & 0 \\
0 & 0 & 0 & 0 & 0 & 0 & 0 & 0
\end{array} \mid \begin{array}{llllllllllllllll}
0 & 0 & 0 & 0 & 0 & 0 & 0 & 0 & 0 & 0 & 0 & 1 & 0 & 0 & 0 & 0 \\
0 & 0 & 0 & 0 & 0 & 0 & 0 & 0 & 0 & 0 & 0 & 0 & 1 & 0 & 0 & 0
\end{array}
\end{aligned}
$$

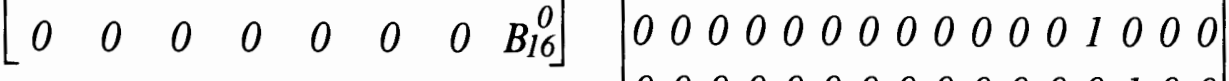

$$
\begin{aligned}
& 000000000000000100 \\
& 0000000000000010
\end{aligned}
$$

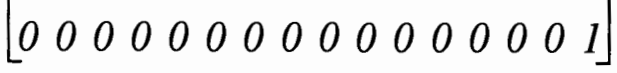


For Stage $2=P_{l 2} * B I(2) * P_{r 2}$ (i.e. set $\mathrm{k}=2, \mathrm{~s}=4, \mathrm{~N}=16$ )
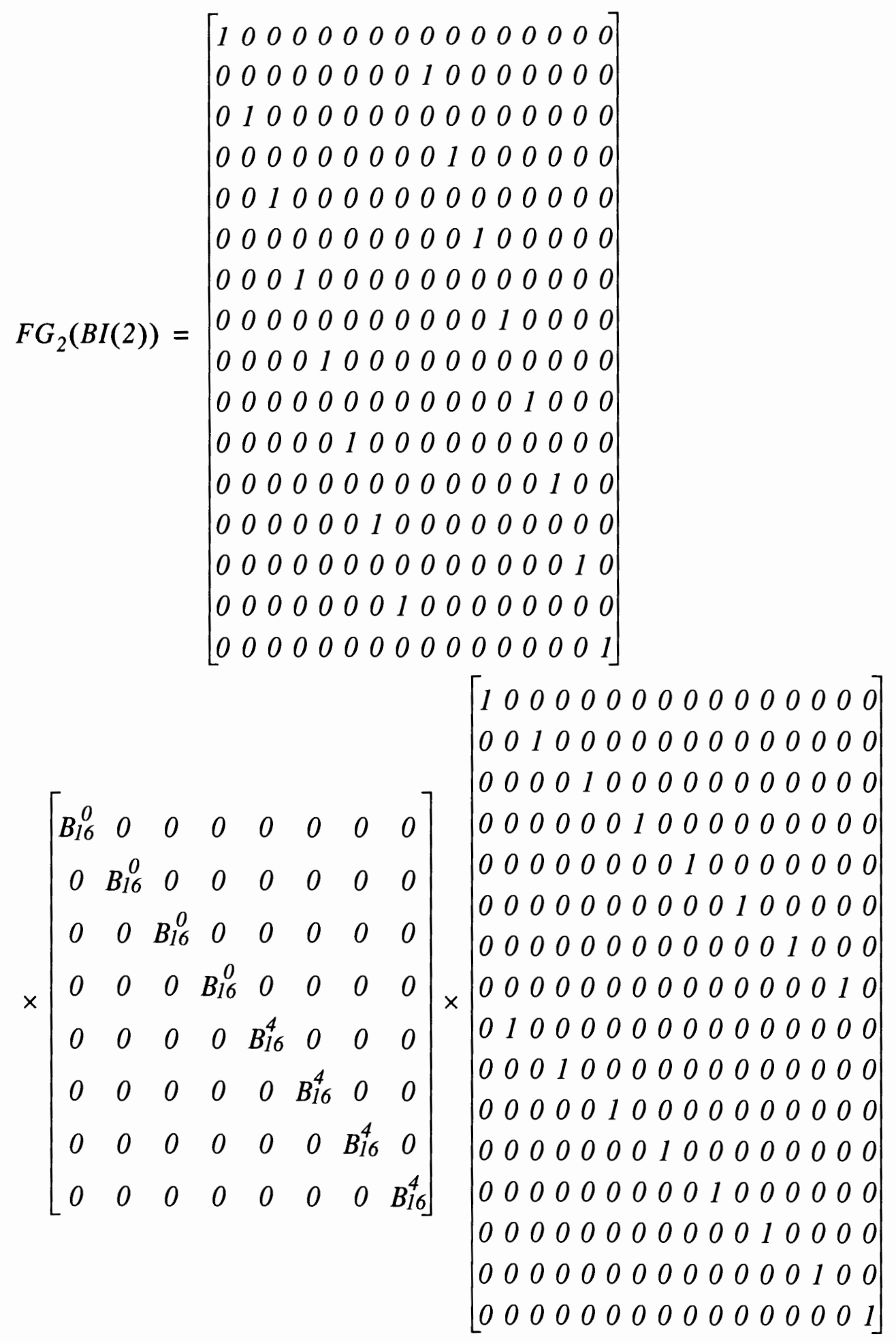
For Stage $3=P_{l 3} * B I(3) * P_{r 3}$ (i.e. set $\mathrm{k}=3, \mathrm{~s}=4, \mathrm{~N}=16$ )

$F G_{3}(B I(3))=\left[\begin{array}{llllllllllllllll}1 & 0 & 0 & 0 & 0 & 0 & 0 & 0 & 0 & 0 & 0 & 0 & 0 & 0 & 0 & 0 \\ 0 & 0 & 0 & 0 & 1 & 0 & 0 & 0 & 0 & 0 & 0 & 0 & 0 & 0 & 0 & 0 \\ 0 & 0 & 0 & 0 & 0 & 0 & 0 & 0 & 1 & 0 & 0 & 0 & 0 & 0 & 0 & 0 \\ 0 & 0 & 0 & 0 & 0 & 0 & 0 & 0 & 0 & 0 & 0 & 0 & 1 & 0 & 0 & 0 \\ 0 & 1 & 0 & 0 & 0 & 0 & 0 & 0 & 0 & 0 & 0 & 0 & 0 & 0 & 0 & 0 \\ 0 & 0 & 0 & 0 & 0 & 1 & 0 & 0 & 0 & 0 & 0 & 0 & 0 & 0 & 0 & 0 \\ 0 & 0 & 0 & 0 & 0 & 0 & 0 & 0 & 0 & 1 & 0 & 0 & 0 & 0 & 0 & 0 \\ 0 & 0 & 0 & 0 & 0 & 0 & 0 & 0 & 0 & 0 & 0 & 0 & 0 & 1 & 0 & 0 \\ 0 & 0 & 1 & 0 & 0 & 0 & 0 & 0 & 0 & 0 & 0 & 0 & 0 & 0 & 0 & 0 \\ 0 & 0 & 0 & 0 & 0 & 0 & 1 & 0 & 0 & 0 & 0 & 0 & 0 & 0 & 0 & 0 \\ 0 & 0 & 0 & 0 & 0 & 0 & 0 & 0 & 0 & 0 & 1 & 0 & 0 & 0 & 0 & 0 \\ 0 & 0 & 0 & 0 & 0 & 0 & 0 & 0 & 0 & 0 & 0 & 0 & 0 & 0 & 1 & 0 \\ 0 & 0 & 0 & 1 & 0 & 0 & 0 & 0 & 0 & 0 & 0 & 0 & 0 & 0 & 0 & 0 \\ 0 & 0 & 0 & 0 & 0 & 0 & 0 & 1 & 0 & 0 & 0 & 0 & 0 & 0 & 0 & 0 \\ 0 & 0 & 0 & 0 & 0 & 0 & 0 & 0 & 0 & 0 & 0 & 1 & 0 & 0 & 0 & 0 \\ 0 & 0 & 0 & 0 & 0 & 0 & 0 & 0 & 0 & 0 & 0 & 0 & 0 & 0 & 0 & 1\end{array}\right]$

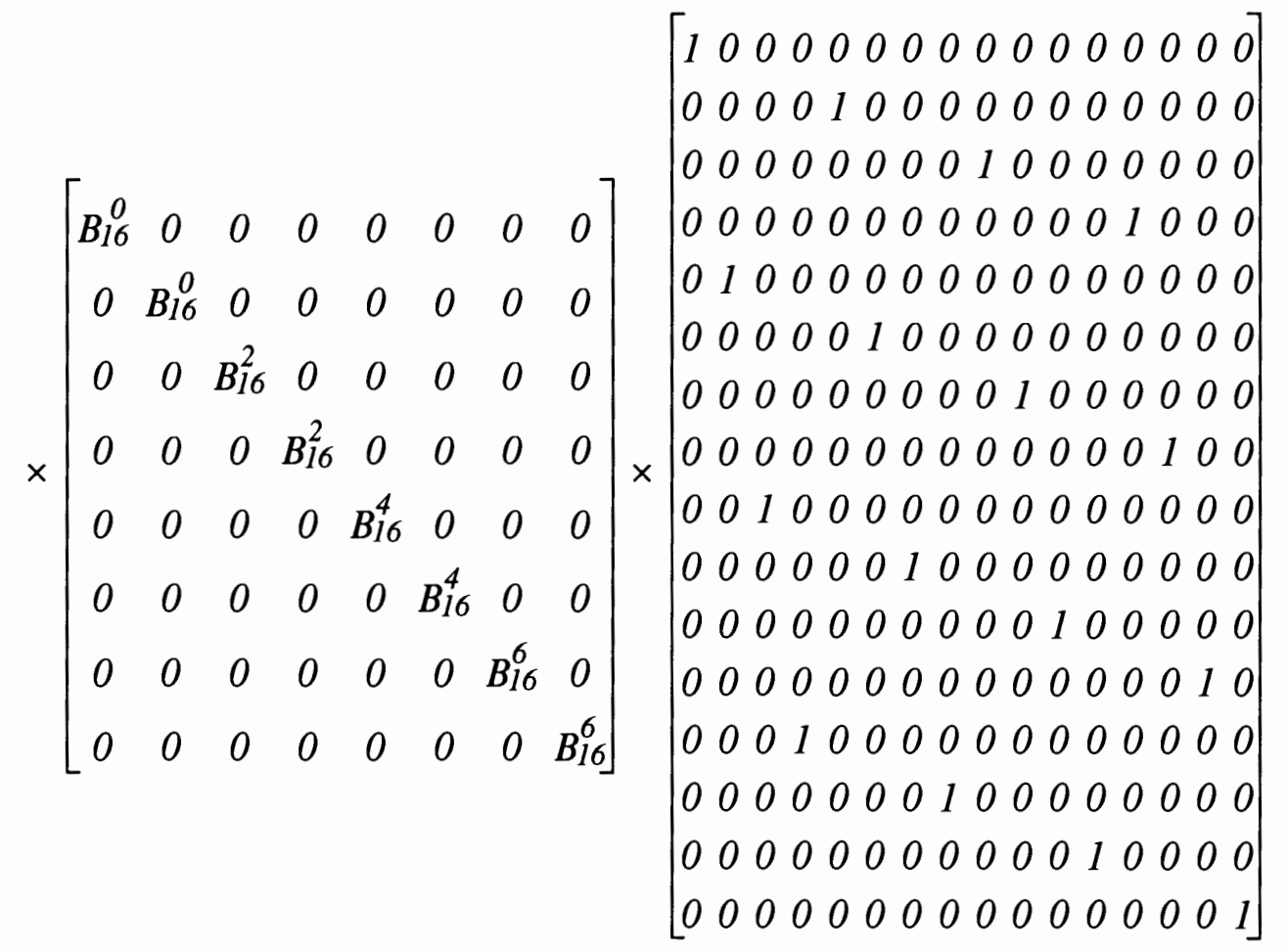


For Stage $4=P_{l 4} * B I(4) * P_{r 4}($ i.e. set $\mathrm{k}=4, \mathrm{~s}=4, \mathrm{~N}=16$ )

$F G_{4}(B I(4))=\left[\begin{array}{llllllllllllllll}1 & 0 & 0 & 0 & 0 & 0 & 0 & 0 & 0 & 0 & 0 & 0 & 0 & 0 & 0 & 0 \\ 0 & 0 & 1 & 0 & 0 & 0 & 0 & 0 & 0 & 0 & 0 & 0 & 0 & 0 & 0 & 0 \\ 0 & 0 & 0 & 0 & 1 & 0 & 0 & 0 & 0 & 0 & 0 & 0 & 0 & 0 & 0 & 0 \\ 0 & 0 & 0 & 0 & 0 & 0 & 1 & 0 & 0 & 0 & 0 & 0 & 0 & 0 & 0 & 0 \\ 0 & 0 & 0 & 0 & 0 & 0 & 0 & 0 & 1 & 0 & 0 & 0 & 0 & 0 & 0 & 0 \\ 0 & 0 & 0 & 0 & 0 & 0 & 0 & 0 & 0 & 0 & 1 & 0 & 0 & 0 & 0 & 0 \\ 0 & 0 & 0 & 0 & 0 & 0 & 0 & 0 & 0 & 0 & 0 & 0 & 1 & 0 & 0 & 0 \\ 0 & 0 & 0 & 0 & 0 & 0 & 0 & 0 & 0 & 0 & 0 & 0 & 0 & 0 & 1 & 0 \\ 0 & 1 & 0 & 0 & 0 & 0 & 0 & 0 & 0 & 0 & 0 & 0 & 0 & 0 & 0 & 0 \\ 0 & 0 & 0 & 1 & 0 & 0 & 0 & 0 & 0 & 0 & 0 & 0 & 0 & 0 & 0 & 0 \\ 0 & 0 & 0 & 0 & 0 & 1 & 0 & 0 & 0 & 0 & 0 & 0 & 0 & 0 & 0 & 0 \\ 0 & 0 & 0 & 0 & 0 & 0 & 0 & 1 & 0 & 0 & 0 & 0 & 0 & 0 & 0 & 0 \\ 0 & 0 & 0 & 0 & 0 & 0 & 0 & 0 & 0 & 1 & 0 & 0 & 0 & 0 & 0 & 0 \\ 0 & 0 & 0 & 0 & 0 & 0 & 0 & 0 & 0 & 0 & 0 & 1 & 0 & 0 & 0 & 0 \\ 0 & 0 & 0 & 0 & 0 & 0 & 0 & 0 & 0 & 0 & 0 & 0 & 0 & 1 & 0 & 0 \\ 0 & 0 & 0 & 0 & 0 & 0 & 0 & 0 & 0 & 0 & 0 & 0 & 0 & 0 & 0 & 1\end{array}\right]$

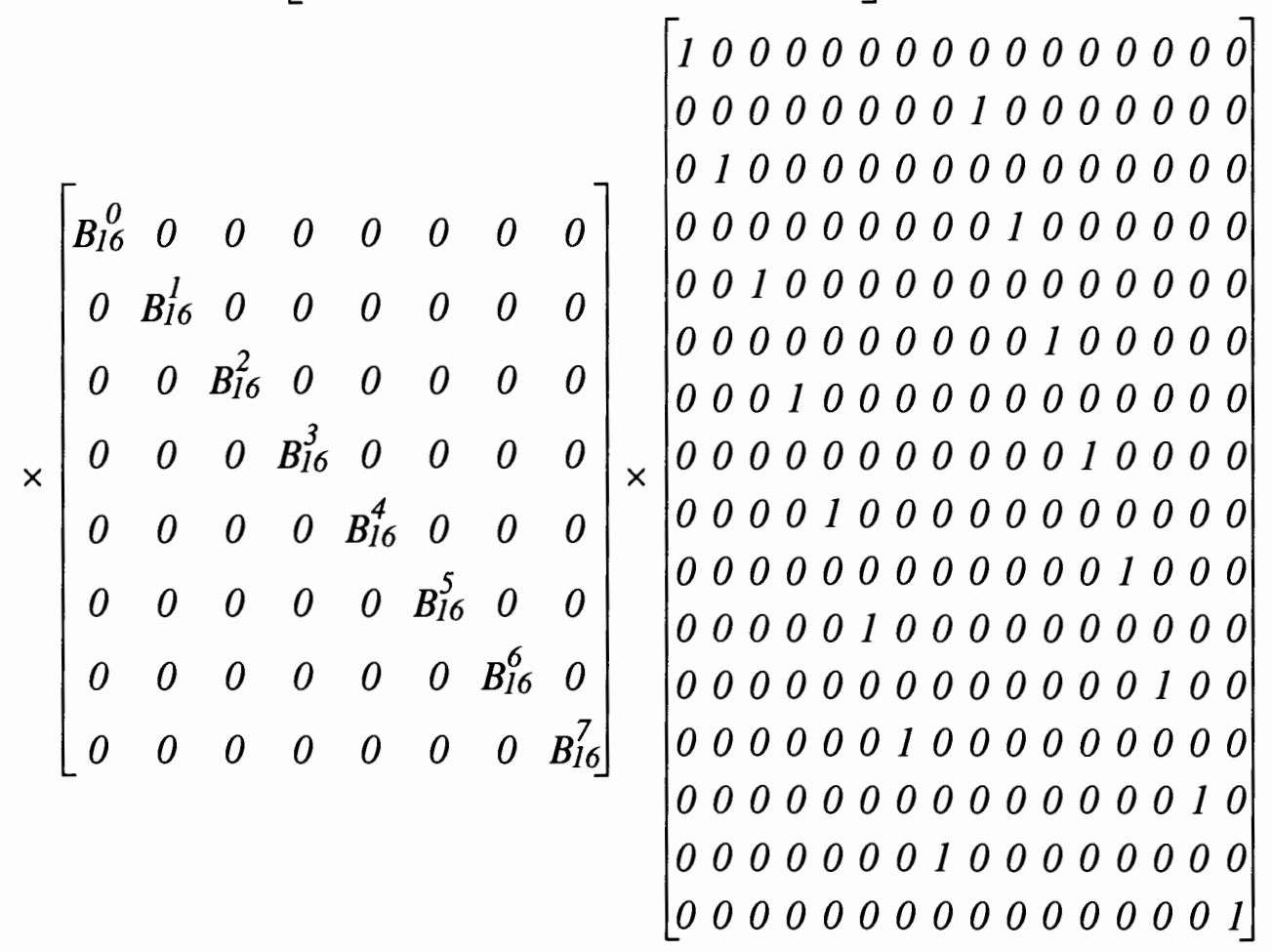




\section{APPENDIX B}

IMPLEMENTATION OF UNIFIED INDEXING TECHNIQUE ON A 2-D 4 BY 4 BI/LO FFT VECTOR MATRIX 


\section{Computation of 2-D 4 by 4 BI/LO FFT Vector Matrix}

The vector-matrix form of unified indexing for the 2-D 4 by $4 \mathrm{BI} / \mathrm{LO}$ FFT implementation can be represented by

$$
\begin{gathered}
\underline{X}_{c}=F G_{s_{2}}\left(B I\left(s_{2}\right)\right) \times F G_{s_{2}-I}\left(B I\left(s_{2}-1\right)\right) \times \ldots \times F G_{I}(B L(1)) \times P_{T} \\
\quad \times F G_{s_{1}}\left(B L\left(s_{1}\right)\right) \times F G_{s_{1}-I}\left(B L\left(s_{1}-1\right)\right) \times \ldots \times F G_{I}(B L(1)) \times x_{b r}^{T}
\end{gathered}
$$

where $x b_{r}$ is an N-point vector in the row-major order of the bit-reverse input array and

$$
P_{T}=P_{r\left(s_{l}+1\right)} \quad \text { and } \quad P_{T}^{-1}=P_{l\left(s_{l}+1\right)}
$$

By substituting Equation (A.2) into Equation (B.1) to compute the bit-reverse input and linear output (BI/LO) of 4 by 4 2-D FFT, it becomes

$$
\begin{aligned}
\underline{X}_{c}=P_{l 2} & \times B I(2) \times P_{r 2} \times P_{l 1} \times B I(1) \times P_{r 1} \times P_{T} \times P_{l 2} \\
& \times B I(2) \times P_{r 2} \times P_{l 1} \times B I(1) \times P_{r I} \times P_{b r} \times \underline{x}_{c}
\end{aligned}
$$

and

$$
P_{T}=P_{r 3} \quad \text { and } \quad P_{T}^{-1}=P_{l 3}
$$

Substituting $P_{T}$ and $P_{T}^{-1}$ into Equation (B.3),

$$
\begin{aligned}
\underline{X}_{c}=P_{T}^{-1} \times P_{l 2} & \times B I(2) \times P_{r 2} \times P_{T} \times P_{T}^{-1} \times P_{l 1} \times B I(1) \times P_{r 1} \times P_{T} \\
& \times P_{l 2} \times B I(2) \times P_{r 2} \times P_{l I} \times B I(1) \times P_{r 1} \times P_{b r} \times \underline{x}_{c}
\end{aligned}
$$

which becomes

$$
\begin{aligned}
\underline{X}_{r}= & P_{l 4} \times B I(2) \times P_{r 4} \times P_{l 3} \times B I(1) \times P_{r 3} \times P_{l 2} \\
& \times B I(2) \times P_{r 2} \times P_{l I} \times B I(1) \times P_{r 1} \times P_{b r} \times \underline{x}_{c}
\end{aligned}
$$

Thus Equation (B.6) is exactly the same as the Equation (A.9) and this shows that the address sequence for 2-D FFT can be transformed into address sequence of 1-D FFT. 
For Stage $1=P_{l l} \times B I(1) \times P_{r l}$ (i.e. set $\mathrm{k}=1, \mathrm{~s}=4, \mathrm{~N}=16$ )

$F G_{I}(B I(1))=\left[\begin{array}{llllllllllllllll}1 & 0 & 0 & 0 & 0 & 0 & 0 & 0 & 0 & 0 & 0 & 0 & 0 & 0 & 0 & 0 \\ 0 & 1 & 0 & 0 & 0 & 0 & 0 & 0 & 0 & 0 & 0 & 0 & 0 & 0 & 0 & 0 \\ 0 & 0 & 1 & 0 & 0 & 0 & 0 & 0 & 0 & 0 & 0 & 0 & 0 & 0 & 0 & 0 \\ 0 & 0 & 0 & 1 & 0 & 0 & 0 & 0 & 0 & 0 & 0 & 0 & 0 & 0 & 0 & 0 \\ 0 & 0 & 0 & 0 & 1 & 0 & 0 & 0 & 0 & 0 & 0 & 0 & 0 & 0 & 0 & 0 \\ 0 & 0 & 0 & 0 & 0 & 1 & 0 & 0 & 0 & 0 & 0 & 0 & 0 & 0 & 0 & 0 \\ 0 & 0 & 0 & 0 & 0 & 0 & 1 & 0 & 0 & 0 & 0 & 0 & 0 & 0 & 0 & 0 \\ 0 & 0 & 0 & 0 & 0 & 0 & 0 & 1 & 0 & 0 & 0 & 0 & 0 & 0 & 0 & 0 \\ 0 & 0 & 0 & 0 & 0 & 0 & 0 & 0 & 1 & 0 & 0 & 0 & 0 & 0 & 0 & 0 \\ 0 & 0 & 0 & 0 & 0 & 0 & 0 & 0 & 0 & 1 & 0 & 0 & 0 & 0 & 0 & 0 \\ 0 & 0 & 0 & 0 & 0 & 0 & 0 & 0 & 0 & 0 & 1 & 0 & 0 & 0 & 0 & 0 \\ 0 & 0 & 0 & 0 & 0 & 0 & 0 & 0 & 0 & 0 & 0 & 1 & 0 & 0 & 0 & 0 \\ 0 & 0 & 0 & 0 & 0 & 0 & 0 & 0 & 0 & 0 & 0 & 0 & 1 & 0 & 0 & 0 \\ 0 & 0 & 0 & 0 & 0 & 0 & 0 & 0 & 0 & 0 & 0 & 0 & 0 & 1 & 0 & 0 \\ 0 & 0 & 0 & 0 & 0 & 0 & 0 & 0 & 0 & 0 & 0 & 0 & 0 & 0 & 1 & 0 \\ 0 & 0 & 0 & 0 & 0 & 0 & 0 & 0 & 0 & 0 & 0 & 0 & 0 & 0 & 0 & 1\end{array}\right]$

$\times\left[\begin{array}{cccccccc}B_{16} & 0 & 0 & 0 & 0 & 0 & 0 & 0 \\ 0 & B_{16}^{0} & 0 & 0 & 0 & 0 & 0 & 0 \\ 0 & 0 & B_{16}^{0} & 0 & 0 & 0 & 0 & 0 \\ 0 & 0 & 0 & B_{16} & 0 & 0 & 0 & 0 \\ 0 & 0 & 0 & 0 & B_{16} & 0 & 0 & 0 \\ 0 & 0 & 0 & 0 & 0 & B_{16}^{0} & 0 & 0 \\ 0 & 0 & 0 & 0 & 0 & 0 & B_{16} & 0 \\ 0 & 0 & 0 & 0 & 0 & 0 & 0 & B_{16}^{0}\end{array}\right] \times\left[\begin{array}{llllllllllllllll}1 & 0 & 0 & 0 & 0 & 0 & 0 & 0 & 0 & 0 & 0 & 0 & 0 & 0 & 0 & 0 \\ 0 & 1 & 0 & 0 & 0 & 0 & 0 & 0 & 0 & 0 & 0 & 0 & 0 & 0 & 0 & 0 \\ 0 & 0 & 1 & 0 & 0 & 0 & 0 & 0 & 0 & 0 & 0 & 0 & 0 & 0 & 0 & 0 \\ 0 & 0 & 0 & 1 & 0 & 0 & 0 & 0 & 0 & 0 & 0 & 0 & 0 & 0 & 0 & 0 \\ 0 & 0 & 0 & 0 & 1 & 0 & 0 & 0 & 0 & 0 & 0 & 0 & 0 & 0 & 0 & 0 \\ 0 & 0 & 0 & 0 & 0 & 1 & 0 & 0 & 0 & 0 & 0 & 0 & 0 & 0 & 0 & 0 \\ 0 & 0 & 0 & 0 & 0 & 0 & 1 & 0 & 0 & 0 & 0 & 0 & 0 & 0 & 0 & 0 \\ 0 & 0 & 0 & 0 & 0 & 0 & 0 & 1 & 0 & 0 & 0 & 0 & 0 & 0 & 0 & 0 \\ 0 & 0 & 0 & 0 & 0 & 0 & 0 & 0 & 1 & 0 & 0 & 0 & 0 & 0 & 0 & 0 \\ 0 & 0 & 0 & 0 & 0 & 0 & 0 & 0 & 0 & 1 & 0 & 0 & 0 & 0 & 0 & 0 \\ 0 & 0 & 0 & 0 & 0 & 0 & 0 & 0 & 0 & 0 & 1 & 0 & 0 & 0 & 0 & 0 \\ 0 & 0 & 0 & 0 & 0 & 0 & 0 & 0 & 0 & 0 & 0 & 1 & 0 & 0 & 0 & 0 \\ 0 & 0 & 0 & 0 & 0 & 0 & 0 & 0 & 0 & 0 & 0 & 0 & 1 & 0 & 0 & 0 \\ 0 & 0 & 0 & 0 & 0 & 0 & 0 & 0 & 0 & 0 & 0 & 0 & 0 & 1 & 0 & 0 \\ 0 & 0 & 0 & 0 & 0 & 0 & 0 & 0 & 0 & 0 & 0 & 0 & 0 & 0 & 1 & 0 \\ 0 & 0 & 0 & 0 & 0 & 0 & 0 & 0 & 0 & 0 & 0 & 0 & 0 & 0 & 0 & 1\end{array}\right]$ 
For Stage $2=P_{l 2} \times B I(2) \times P_{r 2}$ (i.e. set $\mathrm{k}=2, \mathrm{~s}=4, \mathrm{~N}=16$ )

$F G_{2}(B I(2))=\left[\begin{array}{llllllllllllllll}1 & 0 & 0 & 0 & 0 & 0 & 0 & 0 & 0 & 0 & 0 & 0 & 0 & 0 & 0 & 0 \\ 0 & 0 & 0 & 0 & 0 & 0 & 0 & 0 & 1 & 0 & 0 & 0 & 0 & 0 & 0 & 0 \\ 0 & 1 & 0 & 0 & 0 & 0 & 0 & 0 & 0 & 0 & 0 & 0 & 0 & 0 & 0 & 0 \\ 0 & 0 & 0 & 0 & 0 & 0 & 0 & 0 & 0 & 1 & 0 & 0 & 0 & 0 & 0 & 0 \\ 0 & 0 & 1 & 0 & 0 & 0 & 0 & 0 & 0 & 0 & 0 & 0 & 0 & 0 & 0 & 0 \\ 0 & 0 & 0 & 0 & 0 & 0 & 0 & 0 & 0 & 0 & 1 & 0 & 0 & 0 & 0 & 0 \\ 0 & 0 & 0 & 1 & 0 & 0 & 0 & 0 & 0 & 0 & 0 & 0 & 0 & 0 & 0 & 0 \\ 0 & 0 & 0 & 0 & 0 & 0 & 0 & 0 & 0 & 0 & 0 & 1 & 0 & 0 & 0 & 0 \\ 0 & 0 & 0 & 0 & 1 & 0 & 0 & 0 & 0 & 0 & 0 & 0 & 0 & 0 & 0 & 0 \\ 0 & 0 & 0 & 0 & 0 & 0 & 0 & 0 & 0 & 0 & 0 & 0 & 1 & 0 & 0 & 0 \\ 0 & 0 & 0 & 0 & 0 & 1 & 0 & 0 & 0 & 0 & 0 & 0 & 0 & 0 & 0 & 0 \\ 0 & 0 & 0 & 0 & 0 & 0 & 0 & 0 & 0 & 0 & 0 & 0 & 0 & 1 & 0 & 0 \\ 0 & 0 & 0 & 0 & 0 & 0 & 1 & 0 & 0 & 0 & 0 & 0 & 0 & 0 & 0 & 0 \\ 0 & 0 & 0 & 0 & 0 & 0 & 0 & 0 & 0 & 0 & 0 & 0 & 0 & 0 & 1 & 0 \\ 0 & 0 & 0 & 0 & 0 & 0 & 0 & 1 & 0 & 0 & 0 & 0 & 0 & 0 & 0 & 0 \\ 0 & 0 & 0 & 0 & 0 & 0 & 0 & 0 & 0 & 0 & 0 & 0 & 0 & 0 & 0 & 1\end{array}\right]$

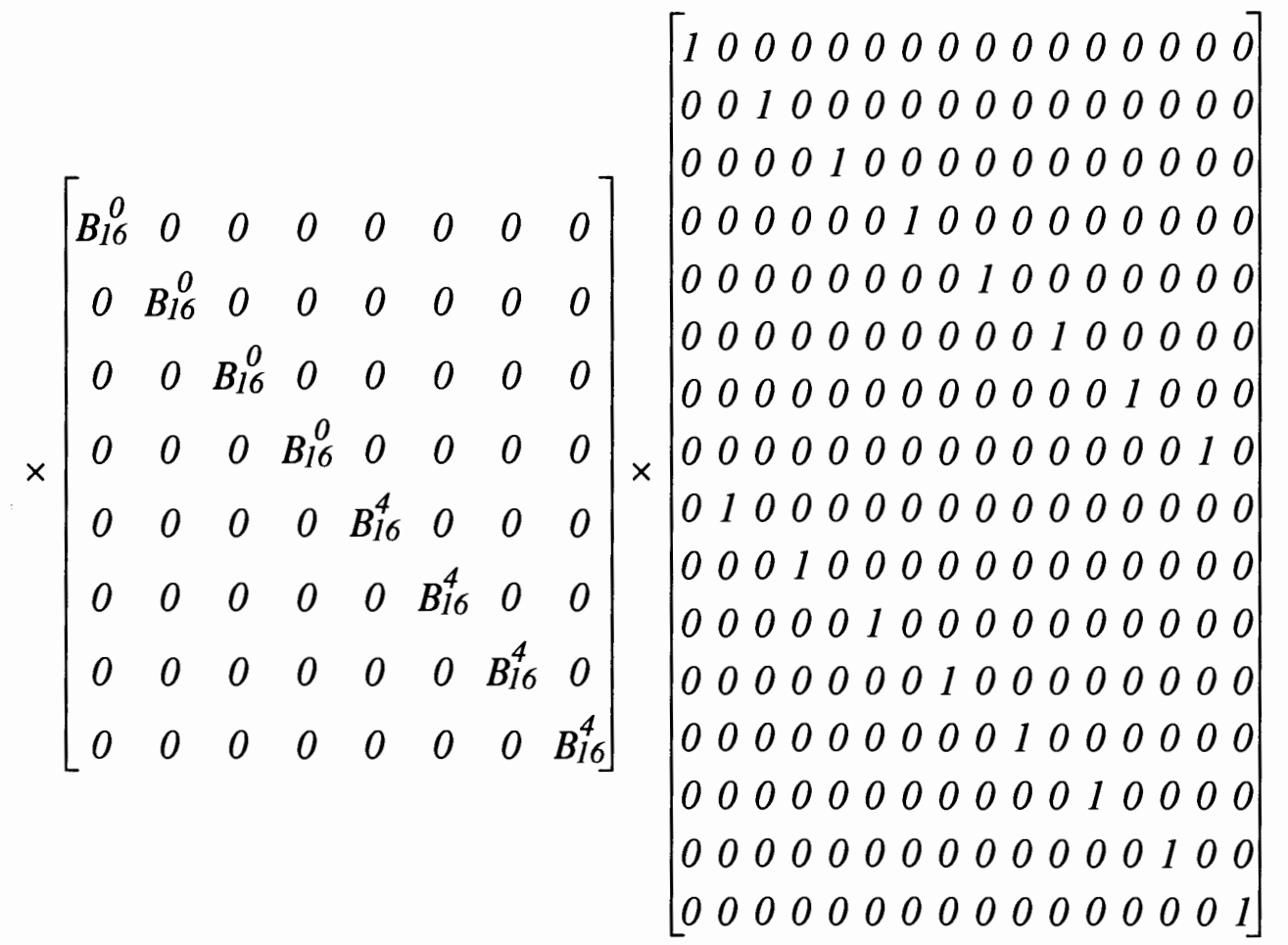


For Stage $3=P_{T}^{-1} \times P_{l 1} \times B I(1) \times P_{r 1} \times P_{T}=P_{l 3} \times B I(3) \times P_{r 3}$

$$
\text { (i.e. set } \mathrm{k}=3, \mathrm{~s}=4, \mathrm{~N}=16 \text { ) }
$$

$F G_{3}(B I(3))=\left[\begin{array}{llllllllllllllll}1 & 0 & 0 & 0 & 0 & 0 & 0 & 0 & 0 & 0 & 0 & 0 & 0 & 0 & 0 & 0 \\ 0 & 0 & 0 & 0 & 1 & 0 & 0 & 0 & 0 & 0 & 0 & 0 & 0 & 0 & 0 & 0 \\ 0 & 0 & 0 & 0 & 0 & 0 & 0 & 0 & 1 & 0 & 0 & 0 & 0 & 0 & 0 & 0 \\ 0 & 0 & 0 & 0 & 0 & 0 & 0 & 0 & 0 & 0 & 0 & 0 & 1 & 0 & 0 & 0 \\ 0 & 1 & 0 & 0 & 0 & 0 & 0 & 0 & 0 & 0 & 0 & 0 & 0 & 0 & 0 & 0 \\ 0 & 0 & 0 & 0 & 0 & 1 & 0 & 0 & 0 & 0 & 0 & 0 & 0 & 0 & 0 & 0 \\ 0 & 0 & 0 & 0 & 0 & 0 & 0 & 0 & 0 & 1 & 0 & 0 & 0 & 0 & 0 & 0 \\ 0 & 0 & 0 & 0 & 0 & 0 & 0 & 0 & 0 & 0 & 0 & 0 & 0 & 1 & 0 & 0 \\ 0 & 0 & 1 & 0 & 0 & 0 & 0 & 0 & 0 & 0 & 0 & 0 & 0 & 0 & 0 & 0 \\ 0 & 0 & 0 & 0 & 0 & 0 & 1 & 0 & 0 & 0 & 0 & 0 & 0 & 0 & 0 & 0 \\ 0 & 0 & 0 & 0 & 0 & 0 & 0 & 0 & 0 & 0 & 1 & 0 & 0 & 0 & 0 & 0 \\ 0 & 0 & 0 & 0 & 0 & 0 & 0 & 0 & 0 & 0 & 0 & 0 & 0 & 0 & 1 & 0 \\ 0 & 0 & 0 & 1 & 0 & 0 & 0 & 0 & 0 & 0 & 0 & 0 & 0 & 0 & 0 & 0 \\ 0 & 0 & 0 & 0 & 0 & 0 & 0 & 1 & 0 & 0 & 0 & 0 & 0 & 0 & 0 & 0 \\ 0 & 0 & 0 & 0 & 0 & 0 & 0 & 0 & 0 & 0 & 0 & 1 & 0 & 0 & 0 & 0 \\ 0 & 0 & 0 & 0 & 0 & 0 & 0 & 0 & 0 & 0 & 0 & 0 & 0 & 0 & 0 & 1\end{array}\right]$

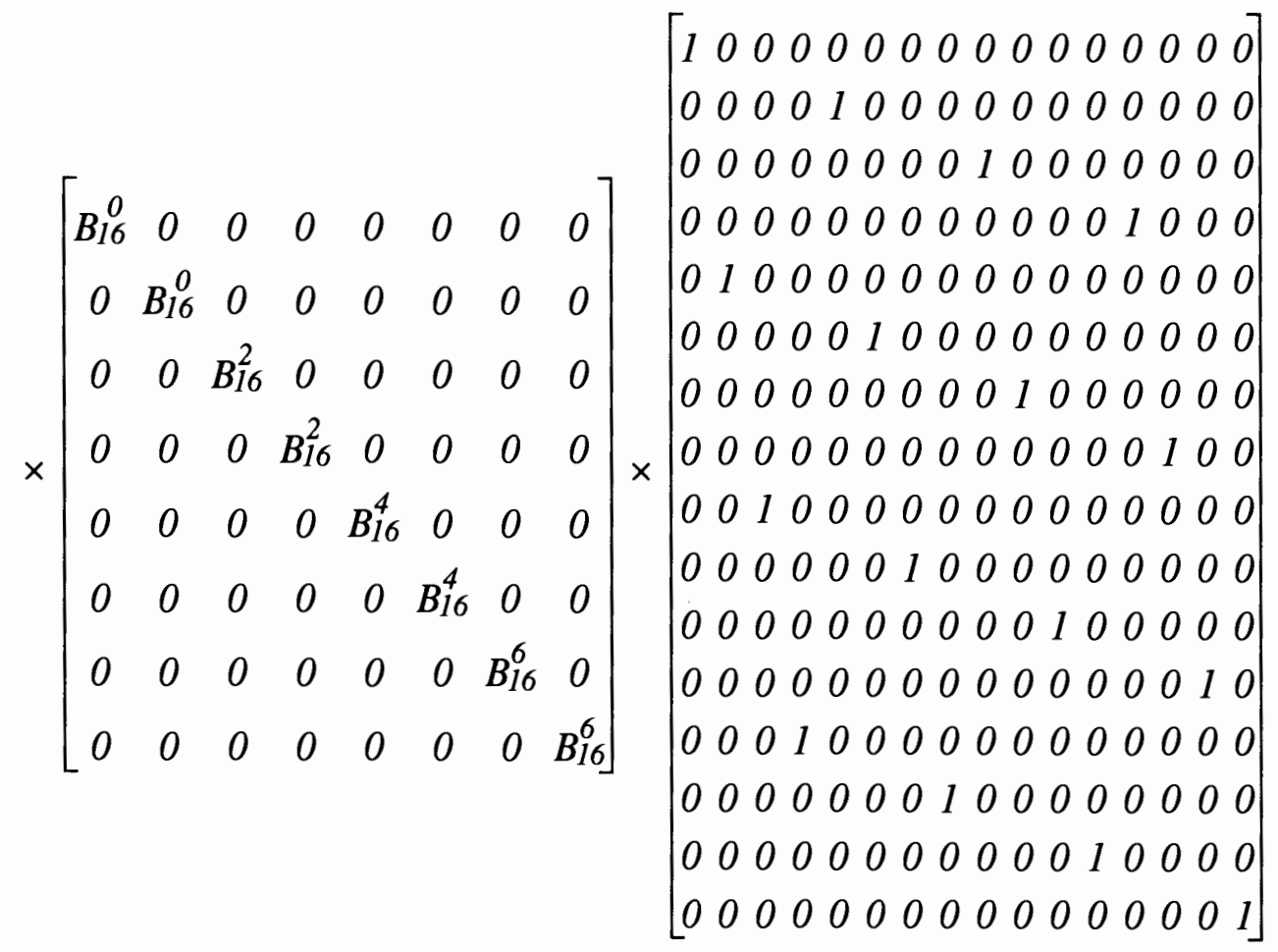


For Stage $4=P_{T}^{-1} \times P_{l 2} \times B I(2) \times P_{r 2} \times P_{T}=P_{l 4} \times B I(4) \times P_{r 4}$

(i.e. set $\mathrm{k}=4, \mathrm{~s}=4, \mathrm{~N}=16$ ).

$F G_{4}(B I(4))=\left[\begin{array}{llllllllllllllll}1 & 0 & 0 & 0 & 0 & 0 & 0 & 0 & 0 & 0 & 0 & 0 & 0 & 0 & 0 & 0 \\ 0 & 0 & 1 & 0 & 0 & 0 & 0 & 0 & 0 & 0 & 0 & 0 & 0 & 0 & 0 & 0 \\ 0 & 0 & 0 & 0 & 1 & 0 & 0 & 0 & 0 & 0 & 0 & 0 & 0 & 0 & 0 & 0 \\ 0 & 0 & 0 & 0 & 0 & 0 & 1 & 0 & 0 & 0 & 0 & 0 & 0 & 0 & 0 & 0 \\ 0 & 0 & 0 & 0 & 0 & 0 & 0 & 0 & 1 & 0 & 0 & 0 & 0 & 0 & 0 & 0 \\ 0 & 0 & 0 & 0 & 0 & 0 & 0 & 0 & 0 & 0 & 1 & 0 & 0 & 0 & 0 & 0 \\ 0 & 0 & 0 & 0 & 0 & 0 & 0 & 0 & 0 & 0 & 0 & 0 & 1 & 0 & 0 & 0 \\ 0 & 0 & 0 & 0 & 0 & 0 & 0 & 0 & 0 & 0 & 0 & 0 & 0 & 0 & 1 & 0 \\ 0 & 1 & 0 & 0 & 0 & 0 & 0 & 0 & 0 & 0 & 0 & 0 & 0 & 0 & 0 & 0 \\ 0 & 0 & 0 & 1 & 0 & 0 & 0 & 0 & 0 & 0 & 0 & 0 & 0 & 0 & 0 & 0 \\ 0 & 0 & 0 & 0 & 0 & 1 & 0 & 0 & 0 & 0 & 0 & 0 & 0 & 0 & 0 & 0 \\ 0 & 0 & 0 & 0 & 0 & 0 & 0 & 1 & 0 & 0 & 0 & 0 & 0 & 0 & 0 & 0 \\ 0 & 0 & 0 & 0 & 0 & 0 & 0 & 0 & 0 & 1 & 0 & 0 & 0 & 0 & 0 & 0 \\ 0 & 0 & 0 & 0 & 0 & 0 & 0 & 0 & 0 & 0 & 0 & 1 & 0 & 0 & 0 & 0 \\ 0 & 0 & 0 & 0 & 0 & 0 & 0 & 0 & 0 & 0 & 0 & 0 & 0 & 1 & 0 & 0 \\ 0 & 0 & 0 & 0 & 0 & 0 & 0 & 0 & 0 & 0 & 0 & 0 & 0 & 0 & 0 & 1\end{array}\right]$

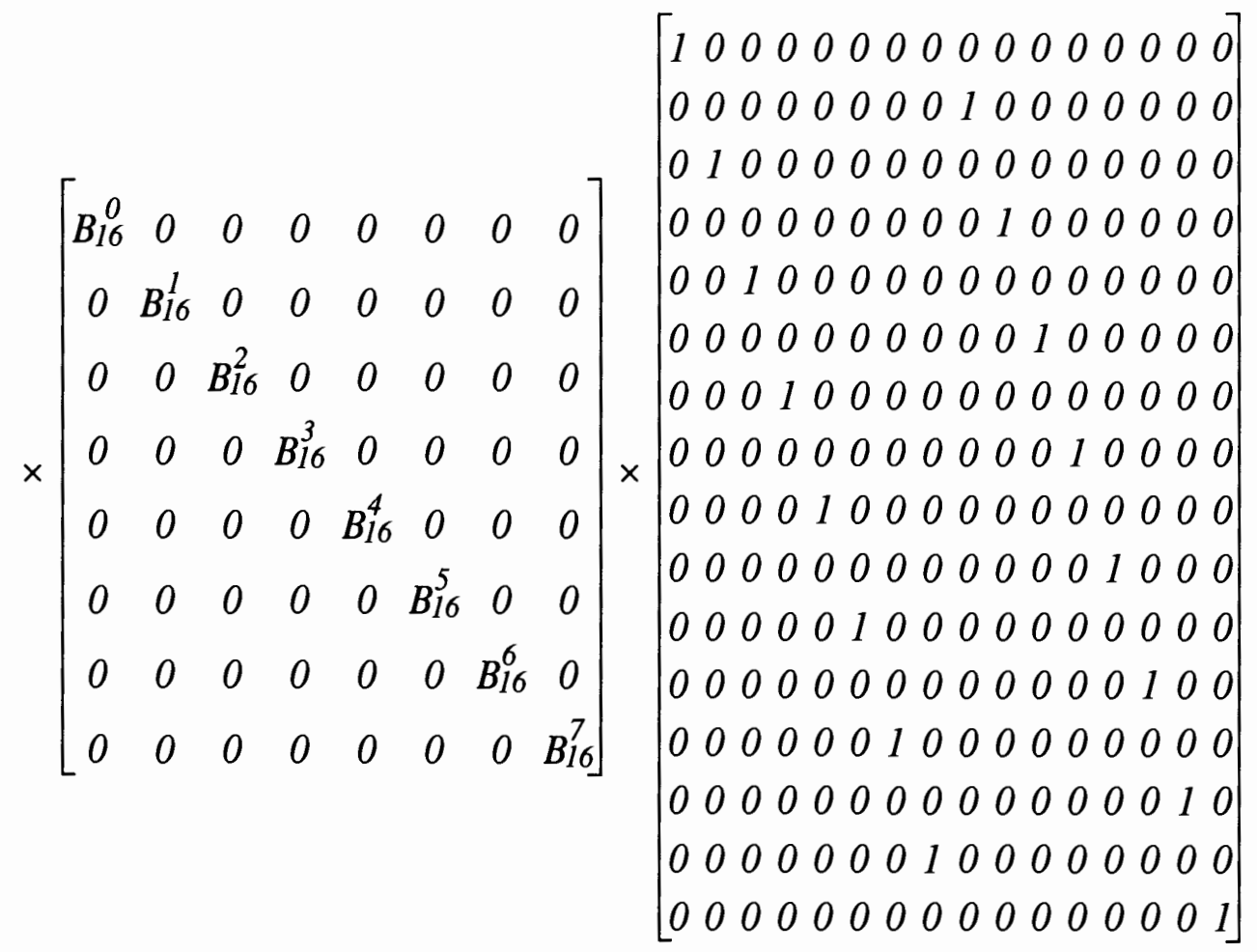




\section{APPENDIX C}

\section{TABLE OF UNIFIED INDEXING SEQUENCES}

FOR PERMUTATION MATRICES 
The indexing sequence for each stage of the permutation matrices from Appendices A and B is listed in Table XVII. To obtain the indexing sequence, the value for left permutation matrix is read row as reference and column as indexing sequence. To read the value for right permutation matrix, it is vice-versa from the left matrix. The left permutation matrix is the transpose of the right permutation matrix. The sequences listed in the table are in decimal value.

Table XVII: Addressing Sequences of 4 by $4 \&$ 16-points 1-D BI/LO FFTs

\begin{tabular}{|c|c|c|c|c|c|c|c|c|c|c|c|c|c|c|c|c|c|c|}
\hline \multirow{3}{*}{$\frac{\text { Stage }}{\text { Index }}$} & \multicolumn{9}{|c|}{ 16-Point 1-D FFT } & \multicolumn{9}{|c|}{4 by 4 2-D FFT } \\
\hline & \multicolumn{3}{|c|}{ S1 } & \multicolumn{2}{|c|}{ S2 } & \multicolumn{2}{|c|}{ S3 } & \multicolumn{2}{|c|}{ S4 } & \multicolumn{3}{|c|}{$\mathbf{R} 1$} & \multicolumn{2}{|c|}{$\mathbf{R 2}$} & \multicolumn{2}{|c|}{ C1 } & \multicolumn{2}{|c|}{$\mathrm{C2}$} \\
\hline & $\mathbf{I}$ & $\mathbf{T}$ & $\mathbf{O}$ & $\mathbf{D}$ & $\mathbf{T}$ & D & $\mathbf{T}$ & $\mathbf{D}$ & $\mathbf{T}$ & $\mathbf{I}$ & $\mathbf{T}$ & $\mathbf{O}$ & D & $\mathbf{T}$ & D & $\mathbf{T}$ & D & $\mathbf{T}$ \\
\hline 0 & 0 & 0 & 0 & 0 & 0 & 0 & 0 & 0 & 0 & 0 & 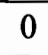 & 0 & 0 & 0 & 0 & 0 & 0 & 0 \\
\hline 1 & 8 & 0 & 1 & 2 & 0 & 4 & 0 & 8 & 0 & 8 & 0 & 1 & 2 & 0 & 4 & 0 & 8 & 0 \\
\hline 2 & 4 & 0 & 2 & 4 & 0 & 8 & 0 & 1 & 0 & 4 & 0 & 2 & 4 & 0 & 8 & 0 & 1 & 0 \\
\hline 3 & 12 & 0 & 3 & 6 & 4 & 12 & 0 & 9 & 4 & 12 & 0 & 3 & 6 & 4 & 12 & 0 & 9 & 4 \\
\hline 4 & 2 & 0 & 4 & 8 & 0 & 1 & 0 & 2 & 0 & 2 & 0 & 4 & 8 & 0 & 1 & 0 & 2 & 0 \\
\hline 5 & 10 & 0 & 5 & 10 & 0 & 5 & 4 & 10 & 2 & 10 & 0 & 5 & 10 & 0 & 5 & 0 & 10 & 0 \\
\hline 6 & 6 & 0 & 6 & 12 & 0 & 9 & 0 & 3 & 0 & 6 & 0 & 6 & 12 & 0 & 9 & 0 & 3 & 0 \\
\hline 7 & 14 & 0 & 7 & 14 & 4 & 13 & 4 & 11 & 6 & 14 & 0 & 7 & 14 & 4 & 13 & 0 & 11 & 4 \\
\hline 8 & 1 & 0 & 8 & 1 & 0 & 2 & 0 & 4 & 0 & 1 & 0 & 8 & 1 & 0 & 2 & 0 & 4 & 0 \\
\hline 9 & 9 & 0 & 9 & 3 & 0 & 6 & 2 & 12 & 1 & 9 & 0 & 9 & 3 & 0 & 6 & 0 & 12 & 0 \\
\hline 10 & 5 & 0 & 10 & 5 & 0 & 10 & 0 & 5 & 0 & 5 & 0 & 10 & 5 & 0 & 10 & 0 & 5 & 0 \\
\hline 11 & 13 & 0 & 11 & 7 & 4 & 14 & 2 & 13 & 5 & 13 & 0 & 11 & 7 & 4 & 14 & 0 & 13 & 4 \\
\hline 12 & 3 & 0 & 12 & 9 & 0 & 3 & 0 & 6 & 0 & 3 & 0 & 12 & 9 & 0 & 3 & 0 & 6 & 0 \\
\hline 13 & 11 & 0 & 13 & 11 & 0 & 7 & 6 & 14 & 3 & 11 & 0 & 13 & 11 & 0 & 7 & 0 & 14 & 0 \\
\hline 14 & 7 & 0 & 14 & 13 & 0 & 11 & 0 & 7 & 0 & 7 & 0 & 14 & 13 & 0 & 11 & 0 & 7 & 0 \\
\hline 15 & 15 & 0 & 15 & 15 & 4 & 15 & 6 & 15 & 7 & 15 & 0 & 15 & 15 & 4 & 15 & 0 & 15 & 4 \\
\hline
\end{tabular}

Legends:
I: Data Input
D: Data input or output sequence
$\mathrm{Ri}$ : Stage of 2-D row FFT

$\mathrm{T}$ : Twiddle factor sequence

Si: Stage of 1-D FFT

Ci: Stage of 2-D column FFT 


\section{APPENDIX D}

THE PROGRAM SOURCE CODE OF WINDSP

GRAPHICS USER INTERFACE

AND FUNCTIONAL BEHAVIORS OF LH9124 INSTRUCTION SET 


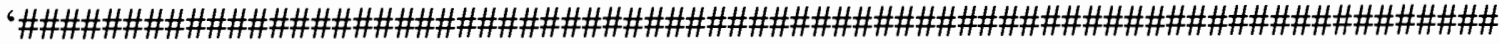

'\# WinDSP.frm

'\# This module is the main program of the WinDSP. It is called the workspace module

'\# where user setup and development application in this module environment. The

'\# modules provide icons of the LH9124 function sets and editor tools that enable user to

'\# link each block together. This module also derive the functional characteristics of each

'\# events when the instruction icon is depressed. As for the LH9124 instruction icons, each

'\# icon will execute the datapath of the LH9124 DSP algorithm and then called the

'\# dynamic link libraries function to execute the complex arithmetic of the DSP function.

'\# Author: Nee-Hua Cho

'\# Revision: 2.0.1

'\# Module is called by starting of the WinDSP program

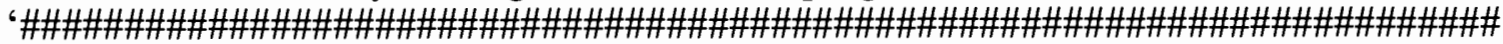

DefInt A-Z

Dim RFlag As Integer

Dim NeedToSave As Integer

Dim MaxVal As Long

Dim MinVal As Long

Dim TxtEnable As Integer

Dim STxtEnable As Integer

Dim TextRect As RECT

Sub Blk_InstHScroll_Changing ()

If ToolPalette_BlkExt.Visible Then ToolPalette BlkExt.Visible $=$ False

Blk_WorkSpace.Left $=$-Blk_InstHScroll.Value

End Sub

Sub Blk_InstHScroll_GotFocus ()

If Pic Work.Visible Then

'If Not BlkToolFlag Then Prepare_New_Icon XArr(ArraySiz), YArr(ArraySiz)

'Tool_Select.Value $=$ False

'CurrentTool $=\&$ HEE

'BlkToolFlag $=$ True

End If

End Sub

Sub Blk_InstVScroll_Changing ()

If ToolPalette BlkExt.Visible Then ToolPalette BlkExt.Visible $=$ False

Blk_WorkSpace.Top = -Blk_InstVScroll.Value

End Sü

Sub Blk_InstVScroll_GotFocus 0

If Pic Work.Visible Then

'If Not BlkToolFlag Then Prepare_New_Icon XArr(ArraySiz), YArr(ArraySiz)

'Tool_Select.Value $=$ False

'CurrentTool $=\&$ HEE 
'BlkToolFlag = True

End If

End Sub

Sub Blk_WorkSpace_DragDrop (Source As Control, X As Single, Y As Single)

Dim Movable As Integer

XTArr $=$ X - DragX: YTArr $=$ Y - DragY

If TypeOf Source Is TextBox Then

TxtUnFocus TxtSelected

Source.Move XTArr, YTArr

TxtFocus TxtSelected

Elself TypeOf Source Is PictureBox Then

If ArraySiz $<0$ Then ArraySiz $=0$ : ArrayCount $=0$

Movable $=$ True

SavedMode $=$ Blk_WorkSpace. DrawMode

'Check to see there is line between the icon to be moved and if it does, erase it 'and update the current line position of the move icon.

For I $=0$ To LineCount -1

If $\operatorname{Hpts}(\mathrm{I}, 0)=$ XArr(ArraySiz) -2 And $\operatorname{Vpts}(\mathrm{I}, 0)=$ YArr(ArraySiz) +16 Then

If XTArr $-2<=\operatorname{Hpts}(I, 1)$ Then

Msg = "Do not drag active icon beyond the left icon"

MsgBox Msg, 16, "Dragging Icon Error"

Movable $=$ False

Exit For

End If

ElseIf Hpts $(\mathrm{I}, 1)=$ XArr(ArraySiz $)+32$ And Vpts $(\mathrm{I}, 1)=$ YArr(ArraySiz $)+24$ Then

If XTArr $+32>=\operatorname{Hpts}(\mathrm{I}, 0)$ Then

Msg = "Do not drag active icon beyond the right icon"

MsgBox Msg, 16, "Dragging Icon Error"

Movable $=$ False

Exit For

End If

ElseIf Hpts(I, 1) = XArr(ArraySiz $)+32$ And Vpts $(I, 1)=$ YArr(ArraySiz $)+8$ Then

'Erase the old Line

XFinish $=\operatorname{Hpts}(I, 0):$ YFinish $=$ Vpts $(I, 0)$

XStart $=$ Hpts(I, 1): YStart $=$ Vpts(I, 1)

If XTArr $+32>=\operatorname{Hpts}(I, 0)$ Then

Msg = "Do not drag active icon beyond the right icon"

MsgBox Msg, 16, "Dragging Icon Error"

Movable $=$ False

Exit For

End If

End If

Next I

If Movable Then

For $\mathrm{I}=0$ To LineCount -1

If Hpts(I, 0) = XArr(ArraySiz) - 2 And Vpts $(I, 0)=$ YArr(ArraySiz) +16 Then

'Erase the old Line 
XFinish $=\operatorname{Hpts}(I, 0):$ YFinish $=$ Vpts $(I, 0)$

XStart $=$ Hpts $(I, 1):$ YStart $=$ Vpts $(I, 1)$

Blk_WorkSpace. .DrawMode $=$ NOT_COPY_PEN

Draw Icon_Line XStart, YStart, XFinish, YF Finish

Blk_WorkSpace. .DrawMode $=$ SavedMode

'Update the line position

Hpts $(\mathrm{I}, 0)=$ XTArr $-2: \operatorname{Vpts}(\mathrm{I}, 0)=$ YTArr +16

XFinish $=$ XTArr $-2:$ YFinish $=$ YTArr +16

Draw_Icon_Line XStart, YStart, XFinish, YFinish

ElseIf $\operatorname{Hpts}(\mathrm{I}, \overline{1})=$ XArr(ArraySiz) +32 And Vpts(I, 1) $=$ YArr(ArraySiz) +24

Then

'Erase the old Line

XFinish $=\operatorname{Hpts}(I, 0):$ YFinish $=\operatorname{Vpts}(I, 0)$

XStart $=$ Hpts(I, 1): YStart $=$ Vpts(I, 1)

Blk_WorkSpace.DrawMode $=$ NOT_COPY_PEN

Draw Icon_Line XStart, YStart, XFïnish, YFinish

Blk_WorkSpace. DrawMode $=$ SavedMode

'Update the line position

Hpts $(\mathrm{I}, 1)=$ XTArr $+32: \operatorname{Vpts}(\mathrm{I}, 1)=$ YTArr +24

XStart $=$ XTArr $+32:$ YStart $=$ YTArr +24

Draw_Icon_Line XStart, YStart, XFinish, YFinish

Elself Hpts $(\mathrm{I}, 1)=$ XArr(ArraySiz $)+32$ And $\operatorname{Vpts}(I, 1)=$ YArr(ArraySiz $)+8$ Then

'Erase the old Line

XFinish $=\operatorname{Hpts}(I, 0):$ YFinish $=\operatorname{Vpts}(I, 0)$

XStart $=$ Hpts $(I, 1):$ YStart $=$ Vpts $(I, 1)$

Blk_WorkSpace.DrawMode = NOT_COPY_PEN

Draw Icon_Line XStart, YStart, XFinish, YFinish

Blk_WorkSpace.DrawMode $=$ SavedMode

'Update the line position

$\operatorname{Hpts}(\mathrm{I}, 1)=\mathrm{XTArr}+32: \operatorname{Vpts}(\mathrm{I}, 1)=$ YTArr +8

XStart $=$ XTArr $+32:$ YStart $=$ YTArr +8

End If

Draw_Icon_Line XStart, YStart, XFinish, YFinish

Next I

XArr(ArraySiz) $=$ XTArr

YArr (ArraySiz) $=$ YTArr

Source.Move XTArr, YTArr

End If

End If

End Sub

Sub Blk_WorkSpace_Load ()

Blk_WorkSpace.Top $=0$

Blk_WorkSpace.Left $=0$

Blk_WorkSpace. BorderStyle $=$ NONE

$\mathrm{XArr}(0)=0: \operatorname{YArr}(0)=0$

PicClip_Icons.ClipY $=0$

PicClip_Icons.ClipX $=160$ 
Tool_Blk6.Picture $=$ PicClip_Icons.Clip

PicClip_Icons.ClipX $=192$

Tool_Blk7.Picture $=$ PicClip_Icons.Clip

PicClip_Icons.ClipX $=224$

Tool_Bl̄k8.Picture $=$ PicClip_Icons.Clip

PicClip_Icons.ClipY $=32$

PicClip_Icons.ClipX $=0$

Tool Blk9.Picture $=$ PicClip_Icons.Clip

PicClip_Icons.ClipX $=32$

Tool_Bl̄k10.Picture $=$ PicClip_Icons.Clip

PicClip_Icons.ClipX $=64$

Tool_Blk11.Picture $=$ PicClip_Icons.Clip

Pic_Work.BorderStyle $=$ NONE

Pic Work.Move 0, 0, 34, 54

End Sub

'This routine consist of three different function, the first does is to transfer the picture from 'the Pic_Work to the WorkSpace and then move Pic_Work to the location where the mouse have previously

'click on.

Sub Blk_WorkSpace_MouseDown (Button As Integer, Shift As Integer, X As Single, Y As Single)

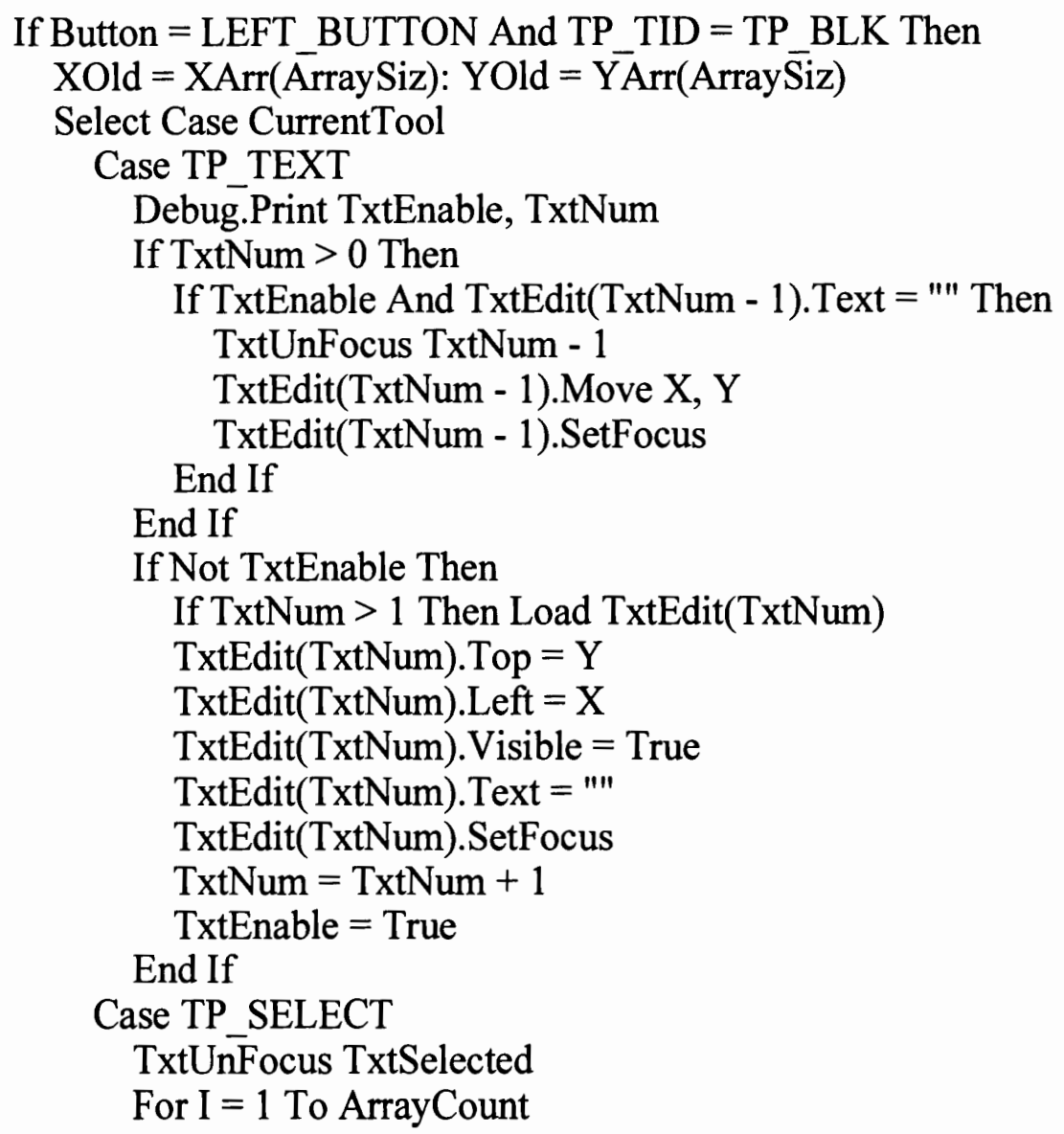


If $(\mathrm{XArr}(\mathrm{I})<=\mathrm{X}$ And $(\mathrm{XArr}(\mathrm{I})+34)>=\mathrm{X})$ And $(\mathrm{YArr}(\mathrm{I})<=\mathrm{Y}$ And $(\mathrm{YArr}(\mathrm{I})$ $+54)>=Y$ ) Then

UnRFocus_Control MainEditor.Pic Work 0 , SRCCOPY)

$\mathrm{R}=$ BitBlt(B)_E_WorkSpace.hDC, XOld, YOld, 34, 54, Pic_Work.hDC, 0 ,

Pic_Work.Picture $=$ LoadPicture ()

Pic_Work.Move XArr(I), YArr(I)

Get_Function_Pic Pic_Work, FuncCode(I, 0)

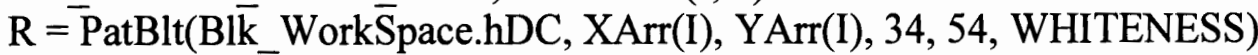

Pic_Box_Paint Pic_Work, Stage(I), FuncCode(I, 0)

ArraySiz $=$ I

Exit Sub

End If

Next I

Case TP_LINE

For $\mathrm{I}=1$ To ArrayCount

If $(\mathrm{XArr}(\mathrm{I})<=\mathrm{X}$ And $(\mathrm{XArr}(\mathrm{I})+34)>=\mathrm{X})$ And $(\mathrm{YArr}(\mathrm{I})<=\mathrm{Y}$ And $(\mathrm{YArr}(\mathrm{I})$ $+54)>=Y$ ) Then

'Check to see which icons is selected so that icon's ID can be properly set

Select Case StartEvent

Case False

IniDraw $=$ True

StartEvent $=$ True

'Store the initial start of the line to draw

StartX $=$ XArr(I) +16

Start $Y=$ YArr(I) +16

'Make the last location equal the starting location

OldX $=$ StartX

OldY $=$ Start $Y$

DsplyFlg1 $=$ FuncCode $(\mathrm{I}, 0)$

ArrayFlg1 $=$ I

Hpts $($ LineCount, 1$)=\mathrm{XArr}(\mathrm{I})+32: \operatorname{Vpts}($ LineCount, 1$)=\mathrm{YArr}(\mathrm{I})+16$

Case True

StartEvent $=$ False

FinDraw $=$ True

DsplyFlg2 $=$ FuncCode $(\mathrm{I}, 0)$

ArrayFlg2 = I

End Select

Hpts $($ LineCount, 0$)=$ XArr(I) $-2: \operatorname{Vpts}($ LineCount, 0$)=$ YArr $(I)+16$

Exit Sub

End If

Next I

Msg = "Invalid -- No Icon selected"

MsgBox Msg, 16, "UnLine Editor"

FinDraw $=$ False: StartEvent $=$ False

Hpts $($ LineCount, 0$)=0: \operatorname{Vpts}($ LineCount, 0$)=0$

Hpts $($ LineCount, 1$)=0: \operatorname{Vpts}($ LineCount, 1) $=0$

If IniDraw Then 
DrawLine Blk_WorkSpace, StartX, StartY, OldX, OldY

IniDraw $=$ False

End If

Case TP_UNLINE

For I $\equiv 1$ To ArrayCount

$+54)>=$ Y) Then

If (XArr(I) $<=$ X And (XArr(I) +34$)>=X$ ) And (YArr(I) $<=$ Y And (YArr(I)

Select Case StartEvent

Case False

IniDraw $=$ True

StartEvent $=$ True

'Store the initial start of the line to draw

StartX $=$ XArr(I) +16

StartY $=$ YArr(I) +16

'Make the last location equal the starting location

OldX $=$ Start X

OldY $=$ Start $Y$

DsplyFlg1 $=$ FuncCode $(\mathrm{I}, 0)$

DisLineX1 $=$ XArr(I) + 32: DisLineY1 $=$ YArr $(\mathrm{I})+16$

Blk_WorkSpace.DrawStyle $=$ DASH

Case True

StartEvent $=$ False

FinDraw $=$ True

DsplyFlg2 $=$ FuncCode $(I, 0)$

DisLineX2 $=$ XArr(I) -2 : DisLineY2 $=$ YArr(I) +16

End Select

Exit Sub

End If

Next I

Msg = "Invalid -- No Icon selected"

MsgBox Msg, 16, "Line Editor"

FinDraw $=$ False: StartEvent $=$ False

DisLineX1 $=0$ : DisLineY1 $=0$

DisLineX2 $=0$ : DisLineY2 $=0$

If IniDraw Then

DrawLine Blk_WorkSpace, StartX, StartY, OldX, OldY

IniDraw $=$ False

\section{End If}

End Select

End If

End Sub

Sub Blk_WorkSpace_MouseMove (Button As Integer, Shift As Integer, X As Single, Y As Single)

$\mathrm{Xpix}=\mathrm{X}$

$\mathrm{YPix}=\mathrm{Y}$

Display_Mouse_Coordinates Xpix, YPix

If StartEvent Then 
DrawLine Blk_WorkSpace, StartX, StartY, OldX, OldY

DrawLine Blk_WorkSpace, StartX, StartY, Xpix, YPix

OldX $=X$

OldY $=\mathrm{Y}$

End If

End Sub

Sub Blk_WorkSpace_MouseUp (Button As Integer, Shift As Integer, X As Single, Y As Single)

If Button $=$ LEFT_BUTTON And TP_TID $=$ TP_BLK Then

Select Case CurrentTool

Case TP LINE

If Not StartEvent And FinDraw Then

'Erase the previous line

DrawLine Blk WorkSpace, StartX, StartY, OldX, OldY

OldX1 $=$ Hpts $($ LineCount, 0$)$

OldY $1=$ Vpts $($ LineCount, 0$)$

StartX $=$ StartX +16

'Check for invalid operation

If StartX > OldX1 Then

to each other"

Msg = "Click data line from left to right Or" \& CRLF \& "Icons are too close

GoTo BlkLine_Err

Elself DsplyFlg1 = TP_DISPLAY And StartX $<$ OldX Then

Msg = "Invalid Operation -- Display icon should be clicked last"

GoTo BlkLine_Err

ElseIf DsplyFlg1 $=$ TP_SIGNAL And DsplyFlg2 $=$ TP_DISPLAY Then

Msg = "Invalid Operation -- No data line should be drawn between" \& CRLF

$\&$ "Signal icon and display icon"

GoTo BlkLine_Err

ElseIf DsplyFlg2 $=$ TP_SIGNAL Then

Msg = "Invalid Operation -- Input to Signal icon is unavailable"

GoTo BlkLine Err

End If

'Swap_Values Hpts(LineCount, 0), Hpts(LineCount, 1), Vpts(LineCount, 0), Vpts(LineCount, 1)

'StartX = Hpts(LineCount, 1)

'StartY $=$ Vpts(LineCount, 1)

'OldX1 = Hpts(LineCount, 0$)$

'OldY1 $=$ Vpts(LineCount, 0 )

If DsplyFlg2 = TP_DISPLAY Then

Else

Vpts $($ LineCount, 1$)=V p t s($ LineCount, 1$)-8$

Vpts $($ LineCount, 1$)=\operatorname{Vpts}($ LineCount, 1$)+8$

End If

'Draw new final line

Start $Y=$ Vpts(LineCount, 1)

Draw_Icon_Line StartX, StartY, OldX1, OldY1 


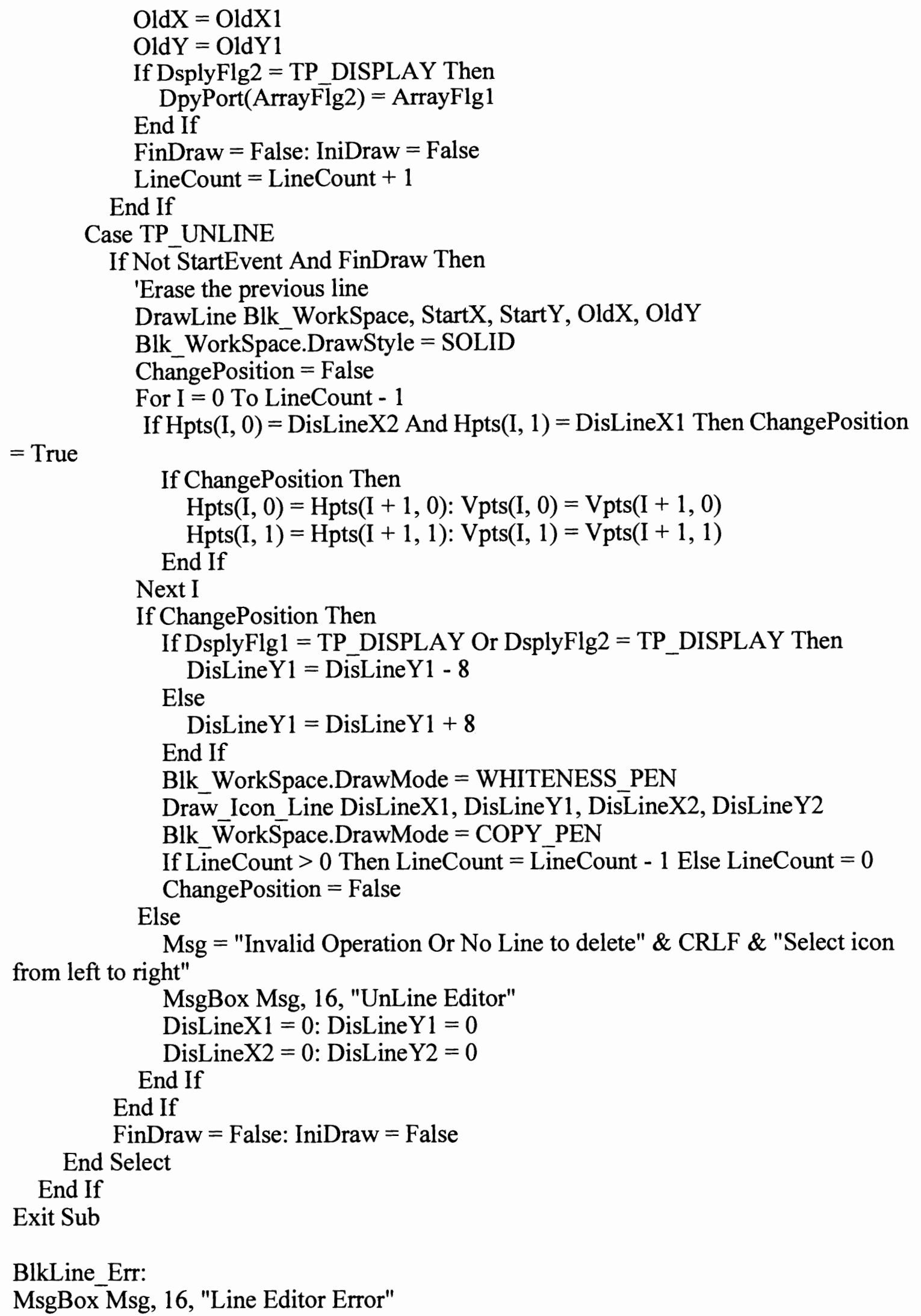


$\operatorname{Hpts}($ LineCount, 0$)=0: \mathrm{Vpts}($ LineCount, 0$)=0$

$\operatorname{Hpts}($ LineCount, 1$)=0: \operatorname{Vpts}($ LineCount, 1$)=0$

FinDraw $=$ False: IniDraw $=$ False

End Sub

Sub Block_Delete_Selection ()

Dim Selecting Ás Integer

Dim PresentTool As Integer

Select Case TP_TID

Case TP_SYS

Present Tool $=$ FuncCode $($ SICount, 1$)$

Selecting $=$ Faise

If SISiz $<=0$ Then

SISiz $=0:$ SICount $=0$

Clipboard.Clear

Exit Sub

End If

$I=0$

Do While I < LCount

If YPts $(I, 0)=$ YSI(SICount $)+16$ And XPts $(I, 0)=X S I($ SICount $)-2$ Then

'Erase the Old Line

XFinish $=$ XPts $(I, 0):$ YFinish $=$ YPts $(I, 0)$

XStart $=$ XPts $(I, 1):$ YStart $=$ YPts $(I, 1)$

Sys_WorkSpace.DrawMode $=$ NOT_COPY_PEN

Draw_Icon_Line XStart, YStart, XFinish, YFinish

Sys_W WorkSpace.DrawMode $=$ COPY_PEN

Selecting $=$ True

Elself YPts $(I, 1)=$ YSI $($ SICount $)+8$ And XPts $(I, 1)=X S I($ SICount $)+32$ Then 'Erase the Old Line

XFinish $=$ XPts $(I, 0):$ YFinish $=$ YPts $(I, 0)$

$X S t a r t=X P t s(I, 1): Y S t a r t=Y P t s(I, 1)$

Sys_WorkSpace.DrawMode $=$ NOT_COPY_PEN

Draw_Icon_Line XStart, YStart, XFinish, YFinish

Sys_W

Selecting $=$ True

ElseIf YPts $(I, 1)=$ YSI $($ SICount $)+24$ And XPts $(I, 1)=X S I($ SICount $)+32$ Then

'Erase the Old Line

XFinish $=$ XPts $(I, 0):$ YFinish $=$ YPts $(I, 0)$

$\mathrm{XStart}=\mathrm{XPts}(\mathrm{I}, 1): \mathrm{YStart}=\mathrm{YPts}(\mathrm{I}, 1)$

Sys_WorkSpace.DrawMode $=$ NOT_COPY_PEN

Draw_Icon_Line XStart, YStart, XFinish, YFinish

Sys_W WorkSpace.. DrawMode $=$ COPY_PEN

Selecting $=$ True

ElseIf YPts $(I, 1)=$ YSI (SICount $)+16$ And XPts $(I, 1)=X S I($ SICount $)+32$ Then

'Erase the Old Line

XFinish $=$ XPts $(I, 0):$ YFinish $=$ YPts $(I, 0)$

$X S t a r t=X P t s(I, 1): Y S t a r t=Y P t s(I, 1)$

Sys_WorkSpace.DrawMode $=$ NOT_COPY_PEN 
Draw_Icon_Line XStart, YStart, XFinish, YFinish

Sys_W WorkSpace.DrawMode $=$ COPY_PEN

Selecting $=$ True

Else

$\mathrm{I}=\mathrm{I}+1$

End If

If Selecting Then

If LCount $>0$ Then LCount $=$ LCount -1 Else LCount $=0$

$\mathrm{J}=\mathrm{I}$

Do While $\mathrm{J}<$ LCount

$\mathrm{XPts}(\mathrm{J}, 0)=\operatorname{XPts}(J+1,0): \operatorname{YPts}(J, 0)=\operatorname{YPts}(J+1,0)$

$\mathrm{XPts}(\mathrm{J}, 1)=\mathrm{XPts}(\mathrm{J}+1,1): \operatorname{YPts}(\mathrm{J}, 1)=\mathrm{YPts}(\mathrm{J}+1,1)$

$\mathrm{J}=\mathrm{J}+1$

Loop

Selecting $=$ False

End If

\section{Loop}

'Remove icon, replace and update the erase icon with the next in the queue For $\mathrm{J}=$ SICount To SISiz -1

$\mathrm{XSI}(\mathrm{J})=\mathrm{XSI}(\mathrm{J}+1): \mathrm{YSI}(\mathrm{J})=\mathrm{YSI}(\mathrm{J}+1)$

FuncCode $(J, 1)=$ FuncCode $(J+1,1)$

$\operatorname{Pass}(J)=\operatorname{Pass}(\mathrm{J}+1)$

If PresentTool $=$ TP_DISPLAY And FuncCode $(J, 1)=T P \_$DISPLAY Then $\operatorname{Pass}(J)=\operatorname{Pass}(J)-1$

Elself PresentTool $=$ TP_SIGNAL And FuncCode $(\mathrm{J}, 1)=$ TP_SIGNAL Then $\operatorname{Pass}(J)=\operatorname{Pass}(J)-1$

ElseIf Present Tool $=$ TP_AIME And FuncCode $(\mathrm{J}, 1)=\mathrm{TP}$ _AIME Then $\operatorname{Pass}(\mathrm{J})=\operatorname{Pass}(\mathrm{J})-1$

ElseIf PresentTool $=$ TP_AGMEM And FuncCode $(J, 1)=$ TP_AGMEM Then $\operatorname{Pass}(J)=\operatorname{Pass}(J)-1$

End If

Next $J$

If $\mathrm{SISiz}>0$ Then

If PresentTool $=$ TP_AIME Then

Aime_Flag = Aime_Flag - 1

If Aime_Flag $<0$ Then Aime Flag $=0$

Elself PresentTool $=$ TP AGMEM Then

$A G \_$Flag $=A G$ Flag -1

If $\overline{A G}$ _Flag $<0$ Then AG_Flag $=0$

ElseIf PresentTool $=$ TP_DISPLAY Then

Disply_Flag $=$ Disply_Flag -1

If Disply_Flag $<0$ Then Disply_Flag $=0$

Elself PresentTool $=$ TP_SIGNAL Then

Signal_Flag $=$ Signal_Flag -1

If Signal_Flag $<0$ Then Signal_Flag $=0$

End If

SICount $=$ SICount -1

$\mathrm{SISiz}=\mathrm{SISiz}-1$ 
End If

If SICount $>=0$ Then

UnRFocus_Control MainEditor.Pic_SysWork

Pic SysWork.Picture $=$ LoadPicture()

If SICount $=0$ Then SICount $=$ SISiz

Pic_SysWork.Move XSI(SICount), YSI(SICount)

If $\overline{\mathrm{SISiz}}=0$ Then

Pic_SysWork.Visible $=$ False

ScreenInfo(ScrnStage) . Changed $=$ False

Else

Get_Function_Pic Pic_SysWork, FuncCode(SICount, 1)

Pic_Box_Paint Pic_SysWork, Pass(SICount), FuncCode(SICount, 1)

RFocus Control MainEditor.Pic SysWork

NESS)

$\mathrm{R}=$ PatBlt(Sys_WorkSpace.hDC, XSI(SICount), YSI(SICount), 34, 54, WHITE-

\section{End If}

End If

Case TP_BLK

PresentTool $=$ FuncCode(ArraySiz, 0$)$

Selecting $=$ False

If ArrayCount $<=0$ Then

Stage_Flag $=0:$ Dpy_Flag $=0$

Sign_Flag $=0$ : ArraySiz $=0$

ArrayCount $=0$

Clipboard.Clear

Exit Sub

End If

'Remove the line on the right of the selected icon and update the Hpts[] \& Vpts[] arrays

$\mathrm{I}=0$

Do While I $<$ LineCount

If $\operatorname{Hpts}(I, 0)=X \operatorname{Arr}($ ArraySiz) -2 And $\operatorname{Vpts}(I, 0)=$ YArr(ArraySiz) +16 Then

XFinish $=\operatorname{Hpts}(I, 0):$ YFinish $=$ Vpts $(I, 0)$

$X$ Start $=\operatorname{Hpts}(I, 1):$ YStart $=$ Vpts $(I, 1)$

Blk_WorkSpace.DrawMode $=$ WHITENESS_PEN

Draw Icon_Line XStart, YStart, XFinish, YFinish

Blk_W WorkSpace.DrawMode $=$ COPY_PEN

Selecting $=$ True

ElseIf Hpts $(I, 1)=$ XArr(ArraySiz $)+32$ And $\operatorname{Vpts}(I, 1)=$ YArr(ArraySiz $)+24$

Then

'Erase the old Line

XFinish $=\operatorname{Hpts}(I, 0):$ YFinish $=\operatorname{Vpts}(I, 0)$

XStart $=\operatorname{Hpts}(I, 1):$ YStart $=$ Vpts $(I, 1)$

Blk_WorkSpace.DrawMode $=$ WHITENESS_PEN

Draw Icon Line XStart, YStart, XFinish, YFinish

Blk_WorkSpace.DrawMode $=$ COPY_PEN

Selecting $=$ True

ElseIf Hpts $(I, 1)=$ XArr(ArraySiz $)+32 \operatorname{And} \operatorname{Vpts}(I, 1)=$ YArr(ArraySiz $)+8$ Then 
'Erase the old Line

XFinish $=\operatorname{Hpts}(\mathrm{I}, 0):$ YFinish $=\mathrm{Vpts}(\mathrm{I}, 0)$

XStart $=$ Hpts $(I, 1):$ YStart $=$ Vpts $(I, 1)$

Blk WorkSpace.DrawMode $=$ WHITENESS_PEN

Draw_Icon_Line XStart, YStart, XFinish, YFinish

Blk_WorkSpace.DrawMode $=$ COPY_PEN

Selecting $=$ True

Else

$\mathrm{I}=\mathrm{I}+1$

End If

If Selecting Then

If LineCount $>0$ Then LineCount $=$ LineCount -1 Else LineCount $=0$

$\mathrm{J}=\mathrm{I}$

Do While $\mathrm{J}<$ LineCount

$\operatorname{Hpts}(J, 0)=\operatorname{Hpts}(\mathrm{J}+1,0): \operatorname{Vpts}(J, 0)=\operatorname{Vpts}(\mathrm{J}+1,0)$

$\operatorname{Hpts}(\mathrm{J}, 1)=\operatorname{Hpts}(\mathrm{J}+1,1): \operatorname{Vpts}(\mathrm{J}, 1)=\operatorname{Vpts}(\mathrm{J}+1,1)$

$\mathrm{J}=\mathrm{J}+1$

Loop

Selecting $=$ False

End If

Loop

'Remove icon, replace and update the erase icon with the next in the queue

For $\mathrm{J}=$ ArraySiz To ArrayCount -1

$\mathrm{XArr}(\mathrm{J})=\mathrm{XArr}(\mathrm{J}+1): \mathrm{YArr}(\mathrm{J})=\mathrm{YArr}(\mathrm{J}+1)$

FuncCode $(J, 0)=$ FuncCode $(J+1,0)$

Stage $(J)=\operatorname{Stage}(J+1)$

If PresentTool $=$ TP_DISPLAY And FuncCode $(\mathrm{J}, 0)=$ TP_DISPLAY Then

Stage $(J)=$ Stage $(\bar{J})-1$

If DpyPort $(J)$ Then DpyPort $(J)=\operatorname{DpyPort}(J+1)$

ElseIf PresentTool $=$ TP_SIGNAL And FuncCode $(J, 0)=$ TP_SIGNAL Then Stage $(J)=\operatorname{Stage}(J)-1$

ElseIf PresentTool $>=\& H 0$ And PresentTool $<=\& H 1 E$ Then

If FuncCode $(J, 0) \diamond \mathrm{TP}$ DISPLAY And FuncCode $(\mathrm{J}, 0) \diamond \mathrm{TP}$ _SIGNAL Then Stage $(J)=\operatorname{Stage}(J)-1$

End If

End If

Next J

If ArrayCount $>0$ Then

If PresentTool $>=0$ And PresentTool $<=\&$ H1E Then

Stage_Flag $=$ Stage Flag -1

If Stage_Flag $<0$ Then Stage_Flag $=0$

Elself PresentTool $=$ TP DISPLAY Then

Dpy_Flag $=$ Dpy_Flag -1

If Dpy_Flag $<0$ Then Dpy_Flag $=0$

Elself PresentTool $=$ TP_SIGNAL Then

Sign_Flag $=$ Sign_Flag -1

If Sign_Flag $<0$ Then Sign_Flag $=0$

End If 


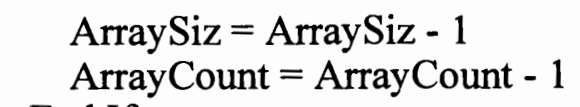

End If

If ArraySiz $>=0$ Then

UnRFocus_Control MainEditor.Pic_Work

Pic Work.Picture $=$ LoadPicture()

If ArraySiz $=0$ Then ArraySiz $=$ ArrayCount

Pic_Work.Move XArr(ArraySiz), YArr(ArraySiz)

If ArrayCount $=0$ Then

Pic Work. Visible $=$ False

ScreenInfo(ScrnStage $)$. Changed $=$ False

Else

Get_Function_Pic Pic_Work, FuncCode(ArraySiz, 0)

Pic_Box_Paint Pic_Work, Stage(ArraySiz), FuncCode(ArraySiz, 0)

RFocus_Control MainEditor.Pic_Work

WHITENESS)

$\mathrm{R}=$ PatBlt(Blk_WorkSpace.hDC, $\mathrm{XArr}$ (ArraySiz), YArr(ArraySiz), 34, 54,

End If

End If

End Select

End Sub

Sub BlockEditor_Close ()

BlockEditor. $. \overline{\text { Visible }}=$ False

End Sub

Sub BlockEditor_Resize ()

If LastBlkWinState = MAXIMIZED Then

TP_TID = TP_MAIN

ScrnStage $=0$

If Pic_Work.Visible Then Prepare_New_Icon XArr(ArraySiz), YArr(ArraySiz)

If ToolPalette_BlkExt.Visible Then ToolPalette_BlkExt.Visible $=$ False

ToolPalette Icons Initialize

ElseIf BlockEditor. $\overline{\text { WindowState }}=$ MAXIMIZED Then

Blk_Root_WkSpace.Move 0, 0, BlockEditor.ClientWidth - Blk_InstVScroll.Width,

BlockEditor.ClientHeight - Blk_InstHScroll.Height

TP_TID $=$ TP_BLK

ScrnStage $=1$

ToolPalette Icons Initialize

Sys_System.Caption = "System"

ScrollBar_Positioning Blk_InstVScroll, Blk_InstHScroll, Blk_WorkSpace,

Blk_Root_Wk $\overline{\text { Space }}$

Pan_NSys.Visible $=$ False

Pan_NSysTxt.Visible $=$ False

If Not Tool_Select.Value Then Tool_Select.Value = True Else Initiate_SignEdit

'If NeedToSave Then Menu_FileSelection_Click MID_SAVE_AS

'If TxtEdit(TxtSelected).Visible Then TxtFocus TxtSelected

End If 
LastBlkWinState $=$ BlockEditor. WindowState

End Sub

Sub Com_MBLK_Click ()

If Not BlockEditor. Visible Then BlockEditor.Visible $=$ True

BlockEditor. WindowState $=$ MAXIMIZED

End Sub

Sub Com_MSYS_Click ()

If Not SystemEditor.Visible Then SystemEditor.Visible $=$ True

SystemEditor. WindowState $=$ MAXIMIZED

End Sub

Sub Cut_Copy_Or_Delete_Selection (Index As Integer)

'This routine check to see which icons to delete in the workspace

Select Case Index

Case MID CUT, MID COPY

If TP TID $=$ TP SYS Then

DeīCutTag $=\overline{\text { Pic }}$ SSysWork.Tag

UnRFocus_Control MainEditor.Pic_SysWork

Clipboard. Clear

Clipboard.SetData Pic_SysWork.Picture, CF_BITMAP

If Index = MID_COPY Then RFocus_Control MainEditor.Pic_SysWork

End If

If TP TID $=$ TP BLK Then

DeilCutTag $=$ Pic_Work.Tag

UnRFocus Control MainEditor.Pic Work

Clipboard.Clear

Clipboard.SetData Pic Work.Picture, CF_BITMAP

If Index = MID_COPY Then RFocus Control MainEditor.Pic_Work

End If

If Index = MID CUT Then Block_Delete_Selection

Case MID DELETE

If TP_TID $=$ TP_BLK Or TP_TID $=$ TP_SYS Then Block_Delete_Selection End Select

End Sub

'As the MousePointer is moved over any portion of the Editing area, 'this routine is called to display the mouse coordinates. The coordinates 'are that of the Work_Space, not the screen coordinates. This routine is 'called by the Work_Space MouseMove.

Sub Display_Mouse_Coordinates (Xpix, YPix)

Pic_StatusArea.CurrentY $=$ Pic_StatusArea.ScaleHeight * .3

'Display X pixel coordinates only if within range of 0-770

If $($ Xpix $>=0)$ And (Xpix $<=$ Main_Width) Then

Pan_XPts.Caption $=$ Format $\$(X$ pix $)$ 
'Pic_StatusArea.CurrentX $=$ Pic_StatusArea.ScaleWidth * .07

'Pic_StatusArea.Print Space\$(A $\bar{b} s(X p i x<10)+\operatorname{Abs}(X p i x<100))+$ Format $\$(X p i x)$; End If

'Display Y pixel coordinates only if within range of 0-460

If (YPix $>=0$ ) And (YPix <= Main_Height) Then

Pan YPts.Caption $=$ Format $\$($ Y Pix $)$

'Pic_StatusArea.CurrentX = Pic_StatusArea.ScaleWidth * .195

'Pic_StatusArea.Print Space $\$(\mathrm{~A} \bar{b} s(Y p i x<10)+\operatorname{Abs}(Y$ pix $<100))+$ Format $\$(Y p i x)$; End If

End Sub

Sub Display_Sys_Icon (SysTNam As Integer)

$\mathrm{SISiz}=\mathrm{SISiz}+1$

Select Case SysTNam

Case TP_COM1 'AIME

Xipos $=0+$ Abs(Sys_WorkSpace.Left $)$

Yipos $=170+$ Abs(Sys_WorkSpace.Top)

FuncCode(SISiz, 1) = TP AIME

Case TP_COM2 'AGMEM

Xipos $=0+$ Abs(Sys_WorkSpace.Left)

Yipos $=204+$ Abs(Sys_WorkSpace.Top)

FuncCode(SISiz, 1) = TP_AGMEM

Case TP_COM3 'SIGNAL

Xipos $=0+$ Abs(Sys_WorkSpace.Left)

Yipos $=236+$ Abs(Sys_WorkSpace.Top)

FuncCode(SISiz, 1) $=\overline{T P}$ _SIGNAL

Case TP_COM4 'DISPLA $\bar{Y}$

Xipos $=0+$ Abs(Sys_WorkSpace.Left $)$

Yipos $=270+$ Abs(Sys_WorkSpace.Top)

FuncCode(SISiz, 1) $=\overline{T P}$ _DISPLAY

End Select

Get_Function_Pic Pic_SysWork, FuncCode(SISiz, 1)

Initial_PicContrl Pic_SysWork, Xipos, Yipos

End Sub

'This routine is to draw the line between the icons, if the icons is in the same level 'vertically a direct line is drawn, else the path of line is divided into half, depending 'on the position of the icons

Sub Draw_Icon_Line (ByVal X1 As Integer, ByVal Y1 As Integer, ByVal X2 As Integer, ByVal Y2 As Integer)

Select Case TP TID

Case TP_SY S

Sys_W WorkSpace.Line $(\mathrm{X} 1, \mathrm{Y} 1)-(\mathrm{X} 1+(\mathrm{X} 2-\mathrm{X} 1) / 2, \mathrm{Y} 1)$

Sys_WorkSpace.Line - $(\mathrm{X} 1+(\mathrm{X} 2-\mathrm{X} 1) / 2, \mathrm{Y} 2)$

Sys_WorkSpace.Line -(X2 - 4, Y2)

Line_Arrow Sys_WorkSpace, X2, Y2 


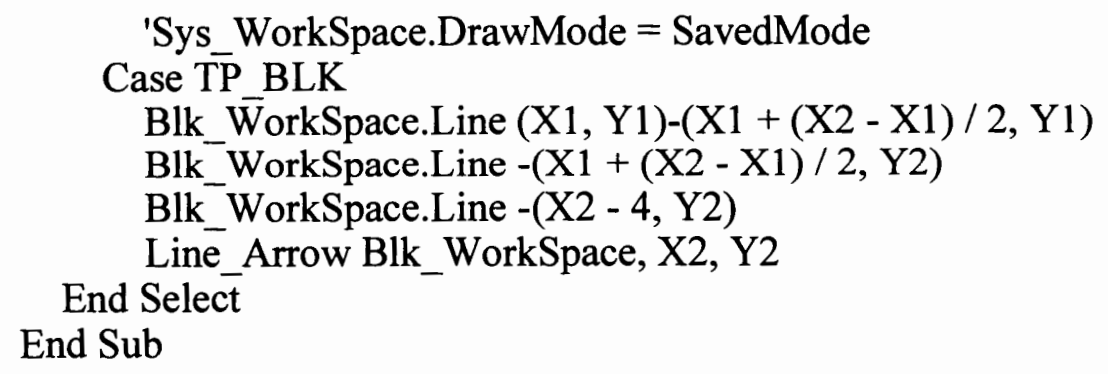

Sub DrawLine (Source1 As Control, X1 As Integer, Y1 As Integer, X2 As Integer, Y2 As Integer)

SavedMode $=$ Source1. DrawMode

Source1. DrawMode $=$ INVERSE

Source1.Line (X1, Y1)-(X2, Y2)

Source1 .DrawMode $=$ SavedMode

End Sub

Sub Exit_System_Click ()

Unloā MainĒ̄itor

End Sub

Sub Form Load 0

'Position the ToolPalette and position the tool icons.

If WindowState $=$ NOMAL Then WindowState $=$ MAXIMIZED

Com_MBLK.Move 0, 0, 34, 34

Com_MSYS.Move $0,34,34,34$

ToolPalette_Icons_Selection.Move 0, 0, 35, 409

Tool Select.Move $0,0,34,34$

Tool Line.Move $0,34,34,34$

Tool UnLine.Move $0,68,34,34$

Tool_Reset.Move $0,102,34,34$

Tool_Text.Move $0,136,34,34$

Tool Com 1.Move $0,170,34,34$

Tool Com2.Move 0, 204, 34, 34

Tool Com3.Move 0, 238, 34, 34

Tool_Com4.Move $0,272,34,34$

Tool_Com5.Move $0,306,34,34$

ToolCom6.Move 0, 340, 34, 34

PicClip_Icons.ClipHeight $=32$

PicClip_Icons.ClipWidth $=32$

PicClip_Icons.ClipY $=192$

'Insert Select icon

PicClip_Icons.ClipX $=0$

Tool_Select.PictureUp $=$ PicClip_Icons.Clip

'Insert Line icon

PicClip_Icons.ClipX $=32$

Tool_Line.PictureUp $=$ PicClip_Icons.Clip

'Insert UnLine icon 
PicClip_Icons.ClipY $=96$

PicClip_Icons.ClipX $=128$

Tool_UnLine.PictureUp = PicClip_Icons.Clip

'Insert Reset/Clear icon

PicClip_Icons.ClipX $=160$

Tool_Reset.PictureUp $=$ PicClip_Icons.Clip

'Insert Text icon

PicClip_Icons.ClipX $=192$

Tool_Text.PictureUp $=$ PicClip_Icons.Clip

'Arranging the drag Icons \& ToolPalette for Sys_level in the Sys_WorkSpace

Sys_WorkSpace_Load

'Arrange the drag Icons \& ToolPalette for Blk_level in the blk_WorkSpace

Blk_WorkSpace_Load

' Initialize the block-level and system-level screen filename to UNTITLED, so a

File.Save operation

' will react just like a File.Save As, the first time.

For $\mathrm{I}=0$ To 2

ScreenInfo(I).Filename $=$ Untitled

Screeninfo(I). Changed $=$ False

Next I

Script FName $=" ' "$

BF_Stage $=0$

'DSPLY_INI_FileName = "BLOCKDSP.INI"

Old_Name $=$ INI_FileName

SFCcount $=0$

LineCount $=0$

LCount $=0$ : Stage_Flag $=0$

Aime_Flag $=0: \mathrm{A} \overline{\mathrm{G}}$ _Flag $=0$

Disply_Flag $=0:$ Signal_Flag $=0$

Dpy_Flag $=0:$ Sign_Flag $=0$

ArraySiz $=0$ : ArrayCount $=0$

SISiz $=0:$ SICount $=0$

TxtNum $=0:$ TxtSelected $=0$

STxtNum $=0:$ STxtSelected $=0$

NeedToSave $=$ False

CurrentTool $=$ TP_SELECT

MainEditorLoade $\bar{d}=$ True

End Sub

Sub Form_Resize ()

Dim Buffers As String * 20

Dim TStrg As String

Dim FPos As Integer

If LastWindowState $=$ NOMAL Then

'Update the Width and the Height of the main screen size

Main_Width $=$ ScaleWidth

Main_Height $=$ ScaleHeight 
'Position the initial selection icons

Work_Space.Move 0, 0, Main_Width, Main_Height

$\mathrm{R}=$ GetWindowsDirectory(Buffers, 20)

FPos $=\operatorname{InStr}(1$, Buffers, $" 1 ")$

TStrg $=$ Mid $\$$ (Buffers, 1, FPos -1$)$

ChDrive TStrg

TStrg $=$ Mid $\$(B u f f e r s$, FPos, R)

ChDir TStrg

If Screen. Height $=7200$ Then

Work_Space.Picture = LoadPicture("3DFiltr1.bmp")

Else

Work_Space.Picture = LoadPicture("3DFiltr2.bmp")

End If

Frm_BlkDsg.Move Main_Width * .35, Main_Height * .8, 94, 52

Frm_Exit.Move Main_Width * .5, Main_Height * .8, 94, 52

Frm_BlkDsg. Visible $=$ True

Frm_Exit.Visible $=$ True

Frm_BlkDsg. SetFocus

ElseIf LastWindowState $=$ MAXIMIZED Then

NOMAL

If SystemEditor. WindowState $=$ MAXIMIZED Then SystemEditor. WindowState $=$ NOMAL

If BlockEditor.WindowState $=$ MAXIMIZED Then BlockEditor. WindowState $=$

End If

LastWindowState $=$ WindowState

End Sub

Sub Form_Unload (Cancel As Integer)

Pic_Work.Picture $=$ LoadPicture()

Pic_Sys Work.Picture $=$ LoadPicture ()

End

End Sub

Sub Frm_BlkDsg_Click ()

TP_TID = TP_BLK

MaīnEditor.Caption = "SHARP WinDSP: [Untitled]"

Frm_Continue

Menu_FileSelection(MID_NEW).Enabled $=$ True

Menu_FileSelection(MID_OPEN).Enabled = True

BlockẼ ditor.Visible $=$ True

BlockEditor. WindowState $=$ MAXIMIZED

End Sub

Sub Frm_Continue ()

Work_Space.Picture $=$ LoadPicture ()

Menu_File.Visible $=$ True

Menu_Edit.Visible $=$ True

Menu_Run.Visible $=$ True 
Menu_Window.Visible $=$ True

Menu Help. Visible = True

Frm_B

Frm Exit. Visible $=$ False

Work__Space.Move 34, 0, Main_Width - 34, Main_Height - 56

Pic_StatusArea.Move 0, Main_Height - 56, Main_Width, 36

ToolPalette_Icons_Selection. Visible $=$ True

Pic_StatusArea.Visible $=$ True

End Sub

Sub Frm_Exit_Click ()

Unloā MainEditor

End Sub

Sub Initial_PicContrl (Sourcel As Control, XIni As Integer, YIni As Integer)

If TP TID $=$ TP SYS Then

$$
\begin{aligned}
& \mathrm{XSI}(\mathrm{SISiz})=\overline{\mathrm{XIni}} \\
& \text { YSI(SISiz) }=\text { YIni } \\
& \text { SICount }=\text { SISiz } \\
& \text { Select Case FuncCode(SICount, 1) } \\
& \text { Case TP_AIME } \\
& \text { Aime_Flag }=\text { Aime_Flag }+1 \\
& \text { Pass }(\overline{\text { SICount }})=\text { Aime_Flag } \\
& \text { Case TP_AGMEM } \\
& \text { AG_Flag }=\text { AG_Flag }+1 \\
& \text { Pass(SICount) }=\text { AG_Flag } \\
& \text { Case TP DISPLAY } \\
& \text { Disply_Flag }=\text { Disply_Flag }+1 \\
& \text { Pass(SICount) }=\text { Disply_Flag } \\
& \text { Case TP_SIGNAL } \\
& \text { Signal_Flag }=\text { Signal_Flag }+1 \\
& \text { Pass(SIC Count) }=\text { Signal_Flag } \\
& \text { Case Else } \\
& \text { Pass(SICount) }=0
\end{aligned}
$$


Sign_Flag $=$ Sign_Flag +1
Stage $($ ArraySiz $)=$ Sign_Flag

Case Else

$$
\text { Stage }(\text { ArraySiz })=0
$$

End Select

If FuncCode(ArraySiz, 0) $<$ TD_VMXM Then SFCcount $=$ SFCcount +1

End If

ScreenInfo(ScrnStage $)$. Changed $=$ True

Tool_Select.Value $=$ True 'Trigger the Select button click event and show depress End $\mathrm{Sub}$

Sub Line_Arrow (Source1 As Control, XCorner As Integer, YCorner As Integer)

Source1.Line (XCorner, YCorner)-(XCorner - 4, YCorner - 4) 'Draw upper slant

Source 1.Line -(XCorner - 4, YCorner + 4)

Source1.Line -(XCorner, YCorner)

End Sub

Sub MaxMinFun (NSTem As Long, Maxi As Long, Mini As Long)

If Inpdat.Rel0 $>=$ Maxi Then

Maxi $=$ Inpdat.Rel 0

ElseIf Inpdat.Re10 $<=$ Mini Then

Mini = Inpdat.Rel0

End If

If Inpdat.Iml0 $>=$ Maxi Then

Maxi $=$ Inpdat. $I m l 0$

ElseIf Inpdat.Iml $0<=$ Mini Then

Mini = Inpdat. $\operatorname{Iml} 0$

End If

If Inpdat.Rel1 $>=$ Maxi Then

Maxi $=$ Inpdat.Rel 1

ElseIf Inpdat.Rel $1<=$ Mini Then

Mini $=$ Inpdat. Rel 1

End If

If Inpdat.Iml1 $>=$ Maxi Then

Maxi $=$ Inpdat.Iml 1

ElseIf Inpdat.Iml $<=$ Mini Then

Mini $=$ Inpdat.Imll

End If

If NSTem $=0$ Then

Outdat.R0T = Inpdat.Rel0: Outdat. $\mathrm{I0T}=$ Inpdat. $I \mathrm{Im} 10$

Outdat.R1T $=0:$ Outdat. I0 $\mathrm{T}=0$

Else

Outdat.R0T $=$ Maxi: Outdat.I0T $=$ Mini

Outdat.R1T $=0$ : Outdat.I1T $=0$

End If

If Inpdat.Rel2 >= Maxi Then

Maxi $=$ Inpdat.Rel2

ElseIf Inpdat.Rel $2<=$ Mini Then 
Mini $=$ Inpdat.Rel2

End If

If Inpdat.Iml $2>=$ Maxi Then

Maxi $=$ Inpdat.Iml2

ElseIf Inpdat.Iml $2<=$ Mini Then

Mini = Inpdat. $\operatorname{Iml} 2$

End If

If Inpdat.Rel3 >= Maxi Then

Maxi $=$ Inpdat. Rel 3

ElseIf Inpdat.Rel3 <= Mini Then

Mini = Inpdat.Rel3

End If

If Inpdat.Iml3 $>=$ Maxi Then

Maxi $=$ Inpdat.Iml3

ElseIf Inpdat.Iml $3<=$ Mini Then

Mini = Inpdat. $\operatorname{Iml} 3$

End If

Outdat.R2T $=$ Maxi: Outdat.I2T $=$ Mini

Outdat.R3T $=0:$ Outdat.I3T $=0$

End Sub

Sub Menu_Edit_Click ()

If Not Tool_Select.Value Then Tool_Select.Value $=$ True

Menu_EditSelection(MID_CUT).Enabled = Pic_Work.Visible Or Pic_SysWork.Visible ible

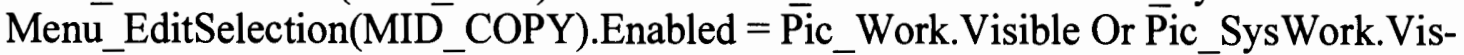

Menu_EditSelection(MID_PASTE).Enabled = Clipboard.GetFormat(CF_BITMAP) And (Pic_Work.Visible Or Pic_SysWork.Visible)

Menu_EditSelection(MID_DELETE).Enabled = Pic_Work.Visible Or Pic_SysWork.Visible

Menu_EditSelection(MID_CLSALL $).$ Enabled $=($ ArrayCount $>0$ Or SISiz $>0)$ And (TP_TID $\diamond$ TP_MAIN)

End Sub

Sub Menu_EditSelection_Click (Index As Integer)

'Comments are to be taken out when necessary

Select Case Index

Case MID_CUT, MID_COPY, MID_DELETE

Cut_Copy_Or_Delete_Selection Index

Case $\bar{M}$ ID PASTE

Paste_ClipBoard_Contents Index

Case MID_EDIT_TXT

Tool_Text.Value $=$ True

TxtEdit(TxtSelected).SetFocus

Case MID_CLSALL

'Clear all items and icons in the workspace

Tool_Reset. Value $=$ True

End Select 
End Sub

Sub Menu_File_Click ()

Menu_FileSelection(MID_NEW).Enabled $=($ TP TID $\diamond$ TP MAIN)

Menu_FileSelection(MID_OPEN).Enabled $=\left(\mathrm{TP}^{-}\right.$TID $\diamond \mathrm{TP}^{-}$MAIN $)$

Menu_FileSelection(MID_SAVE).Enabled $=\left(\mathrm{TP}^{-}{ }_{\text {TID }} \diamond \mathrm{TP}^{-}\right.$MAIN $)$

Menu_FileSelection(MID_SAVE_AS).Enabled $=($ TP_TID $\diamond$ TP_MAIN $)$

Menu_FileSelection(MID_WRITE_SCRIPT).Enabled = Scriptitnow

Select Case TP_TID

Case TP_BLK

'If the Block ToolPalette Extension is visible, hide it

If ToolPalette_BlkExt.Visible Then ToolPalette_BlkExt.Visible $=$ False

'Prepare for draw line. Turn off the Pic_Work Container

If Pic_Work.Visible Then Prepare_New_Icon XArr(ArraySiz), YArr(ArraySiz)

'turn pic work icon OFF

Case TP_SYS

'Prepare for draw line. Turn off the Pic_SysWork Container

If Pic_SysWork.Visible Then Prepare_New_Sys_Icon XSI(SICount), YSI(SICount)

'turn pic_work icon OFF

End Select

End Sub

Sub Menu_FileSelection_Click (Index As Integer)

Dim TemOK As Integer

Dim OrgLeft As Integer

Dim PicFlag As Integer

Script_Path $=$ CurDir

Select Case Index

Case MID_NEW

Scriptitnow $=$ False

Prepare_For_New_Scrn

Case MID_OPĒN

Txt1 $=\overline{\text { "'" }}$

'Check if the Screen has changed since it was last saved.

If ScreenInfo(ScrnStage). Changed Then

'Inform user screen has been modified.

Txt1 $=$ Txt1 + ScreenInfo(ScrnStage $) \cdot$ Filename + CRLF

Txt1 $=$ Txt $1+$ "The project has changed" + CRLF

Txt1 = Txt1 + "Current Path: " + CurDir\$ + CRLF + CRLF

Txt1 = Txt1 + "Do you want to save current changes?"

TemOK = MsgBox(Txt1, 51, "PROJECT HAS CHANGED")

If TemOK $=$ MB_YES Then

Save_Screen MID_SAVE

ElseIf TemOK $=$ MB_CANCEL Then

If Not Tool_Select.Value Then Tool_Select.Value $=$ True Else Initiate_SignEdit Exit Sub

End If

End If 
Open_Screen Index

'TxtEnable $=$ False

If Not Tool_Select.Value Then Tool_Select.Value = True Else Initiate_SignEdit Case MID_SAVE, MID_SAVE_AS

Save Screen Index

If TP_TID $=$ TP_BLK Then

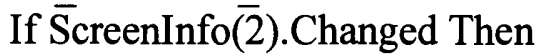

NeedToSave $=$ True

Sys_System_Click

End If

ElseIf TP_TID $=$ TP_SYS Then

If ScreenInfo(1).Changed Then

NeedToSave $=$ True

Sys_System_Click

End If

End If

If Not Tool_Select.Value Then Tool_Select.Value = True Else Initiate_SignEdit Case MID PRINT

If TP_TID $=$ TP_BLK Then

If SystemEditor.Visible Then SystemEditor.Visible $=$ False

MainEditor.PrintForm

If Not SystemEditor.Visible Then SystemEditor.Visible $=$ True

ElseIf TP_TID $=$ TP_SYS Then

If BlockEditor.Visible Then BlockEditor.Visible $=$ False

MainEditor.PrintForm

If Not BlockEditor.Visible Then BlockEditor.Visible $=$ True

End If

If Not Tool_Select.Value Then Tool_Select.Value $=$ True Else Initiate_SignEdit Case MID_WRITE_SCRIPT

Write_Script Index

Case MID EXIT

Unload MainEditor

End Select

End Sub

Sub Menu_HlpSelection_Click (Index As Integer)

If Index $=$ MID_ABOÜT Then

AboutBox.Show Modal

Else

Get_Help Index

End If

End Sub

Sub Menu_WinSelection_Click (Index As Integer)

Select Case Index

Case 1

If TP_TID $=$ TP_MAIN Then

SystemEditor. $\overline{\text { Top }}=0$ 
SystemEditor.Left $=0$

BlockEditor. Top $=21$

BlockEditor.Left $=21$

End If

Case 3

If TP_TID = TP_SYS Then

Sys_WorkSpace.Refresh

'Draw_Icon_Line

Elself TP_TID $=$ TP_BLK Then

Blk_WorkSpace.Refresh

For $\overline{\mathrm{I}}=0$ To LineCount -1

XFinish $=H p t s(I, 0):$ YFinish $=$ Vpts $(I, 0)$

XStart $=$ Hpts $(I, 1):$ YStart $=$ Vpts $(I, 1)$

Draw_Icon_Line XStart, YStart, XFinish, YFinish

Next I

End If

End Select

End Sub

Sub Multiply_Routine_Selection (ByVal NumS As Long, FunTool As Integer, Stages As Integer)

On Error Resume Next

Select Case FunTool

Case TP BFLY16

$\mathrm{R}=\mathrm{B} \overline{\mathrm{fl}} \mathrm{y} 4$ (Inpdat, Outdat, 0 )

If RFlag Then

AutoDSFI $=$ Mag_Factor(Outdat.R0T, Outdat.I0T, AutoDSFI)

AutoDSFI = Mag_Factor(Outdat.R1T, Outdat.I1T, AutoDSFI)

AutoDSFI = Mag_Factor(Outdat.R2T, Outdat.I2T, AutoDSFI)

AutoDSFI = Mag_Factor(Outdat.R3T, Outdat.I3T, AutoDSFI)

End If

Case TP BFLY4

$\mathrm{R}=\mathrm{B}$ ffly4(Inpdat, Outdat, 0 )

AutoDSFI = Mag_Factor(Outdat.R0T, Outdat.I0T, AutoDSFI)

AutoDSFI = Mag_Factor(Outdat.R1T, Outdat.I1T, AutoDSFI)

AutoDSFI = Mag_Factor(Outdat.R2T, Outdat.I2T, AutoDSFI)

AutoDSFI = Mag_Factor(Outdat.R3T, Outdat.I3T, AutoDSFI)

Case TP BFLY2

$\mathrm{R}=\mathrm{B} \overline{\mathrm{fl}} \mathrm{y} 2$ (Inpdat, Outdat, 0 )

If $\mathrm{R}$ Then

MsgBox "Data Overflow or Underflow Error" \& CRLF \& "At stage " \& Format\$(Stages), 16, "Data Computation Error"

End If

AutoDSFI $=$ Mag_Factor(Outdat.R0T, Outdat.I0T, AutoDSFI)

AutoDSFI = Mag_Factor(Outdat.R1T, Outdat.I1T, AutoDSFI)

AutoDSFI = Mag_Factor(Outdat.R2T, Outdat.I2T, AutoDSFI)

AutoDSFI = Mag_Factor(Outdat.R3T, Outdat.I3T, AutoDSFI)

Case TP_BWND4 
$\mathrm{R}=$ Bfly4(Inpdat, Outdat, 1)

AutoDSFI = Mag_Factor(Outdat.R0T, Outdat.I0T, AutoDSFI)

AutoDSFI = Mag_Factor(Outdat.R1T, Outdat.I1T, AutoDSFI)

AutoDSFI = Mag_Factor(Outdat.R2T, Outdat.I2T, AutoDSFI)

AutoDSFI = Mag_Factor(Outdat.R3T, Outdat.I3T, AutoDSFI)

Case TP BWND2

$\mathrm{R}=\mathrm{B} \overline{\mathrm{fl}} \mathrm{y} 2$ (Inpdat, Outdat, 1)

AutoDSFI = Mag_Factor(Outdat.R0T, Outdat.I0T, AutoDSFI)

AutoDSFI = Mag_Factor(Outdat.R1T, Outdat.I1T, AutoDSFI)

AutoDSFI = Mag_Factor(Outdat.R2T, Outdat.I2T, AutoDSFI)

AutoDSFI = Mag_Factor(Outdat.R3T, Outdat.I3T, AutoDSFI)

Case TP BFCT

$\mathrm{R}=\mathrm{B} \overline{\mathrm{fct}} \mathrm{t}($ Inpdat, Outdat, 0 )

AutoDSFI = Mag_Factor(Outdat.R0T, Outdat.I0T, AutoDSFI)

AutoDSFI = Mag_Factor(Outdat.R1T, Outdat.I1T, AutoDSFI)

AutoDSFI = Mag_Factor(Outdat.R2T, Outdat.I2T, AutoDSFI)

AutoDSFI = Mag_Factor(Outdat.R3T, Outdat.I3T, AutoDSFI)

Case TP_BRFT

$\mathrm{R}=\overline{\mathrm{Brft}}($ Inpdat, Outdat $)$

Case TP_BCFIR

$\mathrm{R}=\mathrm{Bcfir}$ (Inpdat, Outdat, rndFlag)

Case TP_BDFIR

$\mathrm{R}=\mathrm{Bd}$ fir(Inpdat, Outdat, rndFlag)

Case TP_BRFIR

$\mathrm{R}=$ Brfir(Inpdat, Outdat, rndFlag)

Case TP BFCT2

$\mathrm{R}=\mathrm{Bfft}($ Inpdat, Outdat, 1$)$

AutoDSFI = Mag_Factor(Outdat.R0T, Outdat.I0T, AutoDSFI)

AutoDSFI = Mag_Factor(Outdat.R1T, Outdat.I1T, AutoDSFI)

AutoDSFI = Mag_Factor(Outdat.R2T, Outdat.I2T, AutoDSFI)

AutoDSFI = Mag_Factor(Outdat.R3T, Outdat.I3T, AutoDSFI)

Case TP_CADD, TP_VADD

$\mathrm{R}=$ Vadd(Inpdat, $\bar{O}$ utdat)

Case TP CMAG

$\mathrm{R}=\mathrm{Cmag}($ Inpdat, Outdat)

Case TP_CMUL

$\mathrm{R}=\overline{\mathrm{Cm}} \overline{\mathrm{m}}($ Inpdat, Outdat)

Case TP CSUB, TP VSUB

$\mathrm{R}=\mathrm{Vsub}($ Inpdat,$\overline{\text { Outdat}})$

Case TP_VABS

Outdat.R0T = Abs(Inpdat.Rel0): Outdat.I0T = Abs(Inpdat.Iml0)

Outdat.R1T $=$ Abs(Inpdat.Rel1): Outdat.I1T $=$ Abs(Inpdat.Iml1)

Outdat.R2T = Abs(Inpdat.Rel2): Outdat.I2T = Abs(Inpdat.Iml2)

Outdat.R3T $=$ Abs(Inpdat.Rel3): Outdat.I3T = Abs(Inpdat.Iml3)

Case TP_VMUL

$\mathrm{R}=\mathrm{Vmul}($ Inpdat, Outdat)

Case TP VMXM

MaxMinFun NumS, MaxVal, MinVal 
Case TP_VNAND

Outdat.R0T $=$ Not (Inpdat.Rel0 And Inpdat.Cox0)

Outdat.I0T $=$ Not (Inpdat.Iml0 And Inpdat.Snx0)

Outdat.R1T $=$ Not (Inpdat.Rel1 And Inpdat.Cox1)

Outdat.I1T $=$ Not (Inpdat.Iml1 And Inpdat.Snx1)

Outdat.R2T $=$ Not (Inpdat.Rel2 And Inpdat.Cox2)

Outdat.I2T = Not (Inpdat.Iml2 And Inpdat.Snx2)

Outdat.R3T $=$ Not (Inpdat.Rel3 And Inpdat.Cox3)

Outdat.I3T = Not (Inpdat.Iml3 And Inpdat.Snx3)

Case TP_VNOR

Outdat.R0T $=$ Not (Inpdat.Rel0 Or Inpdat.Cox0)

Outdat.IOT $=$ Not (Inpdat.Iml0 Or Inpdat.Snx0)

Outdat.R1T $=$ Not (Inpdat.Rel1 Or Inpdat.Cox1)

Outdat.I1T $=$ Not (Inpdat.Iml1 Or Inpdat.Snx1)

Outdat.R2T = Not (Inpdat.Rel2 Or Inpdat.Cox2)

Outdat.I2T = Not (Inpdat.Im12 Or Inpdat.Snx2)

Outdat.R3T = Not (Inpdat.Rel3 Or Inpdat.Cox3)

Outdat.I3T = Not (Inpdat.Iml3 Or Inpdat.Snx3)

Case TP_VPAS, TP_MOVC, TP_MOVD

Outdat.R0T = Inpdat.Rel0: Outdat.IOT = Inpdat. $I m 10$

Outdat.R1T $=$ Inpdat.Rel1: Outdat.I1 T $=$ Inpdat.Iml1

Outdat.R2T = Inpdat.Rel2: Outdat.I2T = Inpdat. $I \mathrm{ml} 2$

Outdat.R3T = Inpdat.Rel3: Outdat.I3T = Inpdat. $I m 13$

Case TP_VXNOR

Outdat.R0T = Not (Inpdat.Rel0 Xor Inpdat.Cox0)

Outdat.I0T $=$ Not (Inpdat.Iml0 Xor Inpdat.Snx0)

Outdat.R1T $=$ Not (Inpdat.Rel1 Xor Inpdat.Cox1)

Outdat.I1 T = Not (Inpdat.Iml1 Xor Inpdat.Snx1)

Outdat.R2T $=$ Not (Inpdat.Rel2 Xor Inpdat.Cox2)

Outdat.I2T $=$ Not (Inpdat.Iml2 Xor Inpdat.Snx2)

Outdat.R3T = Not (Inpdat.Rel3 Xor Inpdat.Cox3)

Outdat.I3T $=$ Not (Inpdat.Iml3 Xor Inpdat.Snx3)

End Select

End Sub

Sub Open_Screen (Index1 As Integer)

On Error Resume Next

Dim LongName As String

Dim Title As String

Dim CDPath As String

If Index 1 = MID_OPEN Then

' Set default filter.

If TP_TID $=$ TP_BLK Then

MainEditor.CMDialog_Edit.Filter $=$ "BDB Files $\left.\left({ }^{*}\right.$. bdb $)\right|^{*}$. BDB"

MainEditor.CMDialog_Edit.DefaultExt = "BDB"

ElseIf TP TID = TP SYS Then

MainEditor.CMDialog_Edit.Filter $=$ "SDB Files $\left.\left({ }^{*}\right.$. sdb $)\right|^{*}$.SDB"

MainEditor.CMDialog_Edit.DefaultExt = "SDB" 
End If

' Display Save As dialog.

MainEditor.CMDialog_Edit.FilterIndex $=1$

If Right(ScreenInfo(ScrnStage).FullPath, 1) $\diamond$ "।" Then

MainEditor.CMDialog_Edit.Filename $=$ ScreenInfo(ScrnStage).FullPath \& "I" \& ScreenInfo(ScrnStage).Filename

End If

' If file has not been saved yet, set common dialog's

' default file name to *.dsp

If ScreenInfo(ScrnStage).Filename = "[Untitled]" Then

MainEditor.CMDialog_Edit.Filename = IIf(TP_TID = TP_BLK, "*.bdb", "*.sdb")

End If

MainEditor.CMDialog_Edit.Action $=1$

' Exit if user cancels the dialog.

If Err $=32755$ Then Exit Sub

Title $=$ MainEditor.CMDialog_Edit.Filetitle

LongName $=$ MainEditor.CMDialog_Edit.Filename

ScreenInfo(ScrnStage).FullPath = Left(LongName, Len(LongName) - Len(Title))

ScreenInfo(ScrnStage) .Filename $=$ MainEditor.CMDialog_Edit.Filetitle

' Adjust for root directory.

If Len(ScreenInfo(ScrnStage).FullPath) $=3$ Then

CDPath $=$ ScreenInfo(ScrnStage $).$ FullPath

Else

CDPath $=$ Left(LongName, Len(LongName) - Len(Title $)-1)$

End If

' Change directories for the editor and the viewer.

ChDir CDPath

End If

MainEditor.MousePointer $=$ HOURGLASS

Write_File_To_Screen ScreenInfo(ScrnStage).FullPath, ScreenInfo(ScrnStage).Filename

MainEditor.MousePointer $=$ DEFAULT

End Sub

Sub Paste_ClipBoard_Contents (Index As Integer)

If Index $=$ MID PASTE Then

If TP_TID $=\overline{\mathrm{T} P} \_\mathrm{BLK}$ Then

ArrayCount $=$ ArrayCount +1

UnRFocus_Control MainEditor.Pic_Work

$\mathrm{R}=\operatorname{BitBlt}(\bar{B} \mathrm{lk}$ _WorkSpace.hDC, XArr(ArraySiz), YArr(ArraySiz), 34, 54,

Pic_Work.hDC, 0, 0, SRCCOPY)

Pic_Work.Picture $=$ LoadPicture()

Pic_Work.Tag $=$ DelCutTag

Pic_Work.Move XArr(ArraySiz) + 15, YArr(ArraySiz) - 15

Pic_Work.Picture $=$ Clipboard.GetData(CF_BITMAP)

If FuncCode(ArraySiz, 0$)>=0$ And FuncCode(ArraySiz, 0$)<=\&$ H1E Then

Stage_Flag $=$ Stage_Flag +1

Stage $($ ArrayCount $)=$ Stage_Flag 
ElseIf FuncCode(ArraySiz, 0) $=$ TP_DISPLAY Then

Dpy_Flag $=$ Dpy_Flag +1

Stage $($ ArrayCount $)=$ Dpy_Flag

ElseIf FuncCode(ArraySiz, 0 ) $=$ TP_SIGNAL Then

Sign_Flag $=$ Sign_Flag +1

Else

Stage $($ ArrayCount $\overline{)}=$ Sign_Flag

Stage (ArrayCount) $=0$

End If

ArraySiz $=$ ArrayCount

Pic_Box_Paint Pic_Work, Stage(ArraySiz), FuncCode(ArraySiz, 0)

RFocus_Control MainEditor.Pic_Work

End If

End If

End Sub

'This routine is to draw a grey line around the Pic_Work or the Pic_SysWork Container 'and draw the blue box around the upper grey box of the DSP function.

Sub Pic_Box_Paint (PicSource1 As Control, BoxNum As Integer, IconID As Integer)

LookÜp_Func_ID PicSource1, IconID

$\mathrm{R}=$ PatBlt $($ PicSourcel.hDC, 2, 33, 28, 18, WHITENESS)

PicSource 1.CurrentX $=0$

PicSource1.Current $Y=31$

If Len(PicSource1.Tag) $<5$ Then

PicSource1.Print " " \& PicSource1.Tag

Else

PicSource1.Print PicSource1.Tag

End If

PicSource1.CurrentX $=5$

PicSource1.Current $Y=40$

PicSource1.Print "["; BoxNum; "]"

End Sub

Sub Pic_IconTool_Highlight (Source2 As Control)

'note this to unhighlight the Picture Icon Tools. These controls

'must get their outline drawn on the picture box they are inside

UnWide $=$ Source 2 . Width

UnHigh $=$ Source2.Height

Source2.Line $(0,0)$-(UnWide, 1), \&HFFFFFF, BF

Source2.Line (0, 0)-(1, UnHigh), \&HFFFFFF, BF

Source2.Line (UnWide, UnHigh)-(0, UnHigh - 3), \&H808080, BF

Source 2.Line (UnWide, UnHigh - 3)-(1, UnHigh - 4), \&H808080, BF

Source2.Line (UnWide, UnHigh)-(UnWide - 3, 0), \&H808080, BF

Source2.Line (UnWide - 3, UnHigh)-(UnWide - 4, 1), \&H808080, BF

End Sub

Sub Pic_StatusArea_Paint () 
'Highlighting the StatusArea

Pic_IconTool_Highlight Pic_StatusArea

'Positioning the command Button

Sys_System.Move Main_Width - 290, 4, 60, 26

Start_System.Move Main_Width - 220, 4, 60, 26

Stop_System.Move Main_Width - 150, 4, 60, 26

Exit_System.Move Main_Width - 80, 4, 60, 26

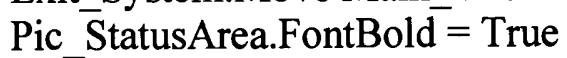

'Display X \& Y mouse coordinates labels, " $\mathrm{X}=$ " and "Y="

Pic_StatusArea.CurrentY $=$ Main_Height * 02

Pic_StatusArea.CurrentX $=$ Main_Width * .01

Pic StatusArea.Print "X=";

Pic_StatusArea.CurrentX $=$ Main_Width * .115

Pic_StatusArea.Print "Y=";

'Position the Panel for X \& Y coordinates

Pan_XPts.Top $=$ Main_Height $* .015$

Pan_XPts.Left $=$ Main_Width ${ }^{*} .034$

Pan_YPts.Top $=$ Main_Height $* .015$

Pan_YPts.Left $=$ Main_Width * 14

Pan_NSysTxt.Top $=$ Main_Height $* .015$

Pan_NSysTxt.Left $=$ Main_Width ${ }^{*} .23$

Pan_NSys.Top $=$ Main_Height $* .015$

Pan_NSys.Left $=$ Main_Width $* .49$

\section{End Sub}

Sub Pic_SysWork_DblClick ()

If TP_TID = TP_SYS Then

XInit $=$ XSI(SICOunt): YInit $=$ YSI (SICount)

If Pic_SysWork.Visible Then Prepare_New_Sys_Icon XInit, YInit

Select Case FuncCode(SICount, 1)

Case TP_AIME

CenterForm SignalEditor

SignalEditor.Show Modeless

Case TP_AGMEM

CenterForm AGMEM Editor

AGMEM Editor.Show Modal

Case TP_DISPLAY

CenterForm BGraph

BGraph.Show Modeless

End Select

End If

End Sub

Sub Pic_SysWork_MouseDown (Button As Integer, Shift As Integer, X As Single, Y As Single)

If TP_TID $=$ TP_SYS Then

Pic_SysWork.Drag 1

$\operatorname{DragX}=\mathrm{X}$ 
End If

$\operatorname{Drag} \mathrm{Y}=\mathrm{Y}$

End Sub

Sub Pic_SysWork_MouseUp (Button As Integer, Shift As Integer, X As Single, Y As Single)

If TP TID $=$ TP_SYS Then Pic_SysWork.Drag 2

End Sū

Sub Pic_SysWork_Paint ()

Pic_SysWork.Line $(1,1)-(30,31)$, QBColor(1), B

Pic_SysWork.Line (1,31)-(30, 52), QBColor(8), B

Pic_Box_Paint Pic_SysWork, Pass(SICount), FuncCode(SICount, 1)

RFocus_Control MainEditor.Pic_SysWork

End Sub

Sub Pic_Work_DblClick ()

If TP_TID $=$ TP_BLK Then

XInit $=$ XArr(ArraySiz): YInit $=$ YArr(ArraySiz)

If Pic_Work.Visible Then Prepare_New_Icon XInit, YInit

Select Case FuncCode(ArraySiz, 0)

Case TP SIGNAL CenterForm XGen

XGen.Show Modal

Case TP_DISPLAY

CenterForm BGraph

BGraph.Show Modal

Case Else

CenterForm SignalEditor

SignalEditor.Show Modal

End Select

End If

End Sub

Sub Pic_Work_MouseDown (Button As Integer, Shift As Integer, X As Single, Y As Single)

If TP_TID $=$ TP_BLK Then

Pic Work.Drä 1

Drag $\mathrm{X}=\mathrm{X}$

$\operatorname{Drag} Y=Y$

End If

End Sub

Sub Pic_Work_MouseUp (Button As Integer, Shift As Integer, X As Single, Y As Single)

If TP_TID = TP_BLK Then Pic_Work.Drag 2

End Sub

Sub Pic_Work_Paint () 
'Paint the Top square with blue color

Pic Work.Line $(1,1)-(30,31)$, QBColor(1), B

'Paint the Bottom square with gray color

Pic Work.Line $(1,31)-(30,52)$, QBColor $(8), \mathrm{B}$

'Update the icon information

Pic_Box_Paint Pic_Work, Stage(ArraySiz), FuncCode(ArraySiz, 0)

'Highlight the icon

RFocus_Control MainEditor.Pic_Work

End Sub

Sub Prepare_For_New_Scrn ()

Dim TemōK Âs Integer

Txtl = "'"

'Check if the Screen has changed since it was last saved.

If ScreenInfo(ScrnStage). Changed Then

'Inform user screen has been modified.

Txt1 $=$ Txt1 + ScreenInfo(ScrnStage $)$.Filename + CRLF

Txt1 $=$ Txt1 + "The project has changed" + CRLF

Txt1 = Txt1 + "Current Path:" + A_TAB + CurDir\$ + CRLF + CRLF

Txtl = Txt1 + "Do you want to save current changes?"

TemOK = MsgBox(Txt1, 51, "PROJECT HAS CHANGED")

If TemOK $=$ MB_YES Then

Save_Screen MID_SAVE

ElseIf TemOK $=$ MB_CANCEL Then

Exit Sub

End If

End If

'Clear the screen and delete all variables

Initialize_All_Variables TP_TID

'Initialize the variables to default value

ScreenInfo(ScrnStage) . Filename $=$ Untitled

ScreenInfo(ScrnStage) . Changed $=$ False

'Reset the title on the editors titlebar

Caption = "SHARP WinDSP: [Untitled] "

End Sub

If Not Tool_Select.Value Then Tool_Select.Value= True Else Initiate_SignEdit

'Prepare the new function icon for the pic_work and copy the existing image of the pic_work

'to the blk_workspace and clear the picture in pic_work.

Sub Prepare_New_Icon (X As Integer, Y As Integer)

UnRFocus_Control MainEditor.Pic_Work

$\mathrm{R}=$ BitBlt(Blk_WorkSpace.hDC, $\mathrm{X}, \mathrm{Y}, 34,54$, Pic_Work.hDC, 0, 0, SRCCOPY)

MainEditor $!$ Pic_Work.Visible $=$ False

MainEditor!Pic_Work.Picture $=$ LoadPicture()

End Sub 
'Prepare the new function icon for the pic_SysWork and copy the existing image of the pic_SysWork

'to the Sys_workspace and clear the picture in pic_SysWork.

Sub Prepare_New_Sys_Icon (X As Integer, Y As Integer)

UnRFocus Control MainEditor.Pic_SysWork

$\mathrm{R}=$ BitBlt(Sys_WorkSpace.hDC, X, Y, 34, 54, Pic_SysWork.hDC, 0, 0, SRCCOPY)

MainEditor!Pic_SysWork.Visible $=$ False

MainEditor!Pic_SysWork.Picture $=$ LoadPicture ()

End Sub

Sub Process IO Data (FunInProgress As Integer, Passes As Integer)

Dim EData Âs Integer

Dim FNumA As Integer

Dim FNumB As Integer

Dim FNumC As Integer

Dim FileNuml As Integer

Dim FileNum2 As Integer

Dim FileNum3 As Integer

Dim CoFlag As Integer

Dim MX1 As Integer

Dim MY1 As Integer

Dim MX2 As Integer

Dim MY2 As Integer

Dim Varl As Integer

Dim Var2 As Integer

Dim Var3 As Integer

Dim J0 As Integer

Dim Datem As Long

Dim Cotem As Long

Dim MemPos As Long

Dim VarTemp As String * 1

Dim RAStrg As String

Dim CAStrg As String

Dim WAStrg As String

On Error Resume Next

Get_Ini_WorkDirEnv

$\mathrm{NS}=0$

'Opening the address pattern files

RAStrg $=$ RTrim $\$\left(R A \_T x t\right) \&$ ".AG2"

CAStrg $=$ RTrim $\$\left(\mathrm{CA}_{-}^{-} \mathrm{Txt}\right) \&$ ".AG2"

WAStrg $=$ RTrim $\$($ WA_Txt $) \&$ ".AG2"

If FunInProgress $=$ TP_ $B$ BLLY16 Then

$\mathrm{EData}=16$

ReDim RRMX(16): ReDim RYMX(16)

ReDim CRTem(16): ReDim CYTem(16)

ReDim RTem(16): ReDim RTem(16)

Else 
$\mathrm{EData}=4$

ReDim RRMX(4): ReDim RYMX(4)

ReDim CRTem(4): ReDim CYTem(4)

ReDim RTem(4): ReDim YTem(4)

End If

FileNum1 $=$ FreeFile

Open RAStrg For Binary Access Read As \#FileNum1

FileNum2 = FreeFile

Open WAStrg For Binary Access Read As \#FileNum2

If Not AutoMan Then

IPStrg = RTrim\$(IP_Txt) \& ".Dat"

OPStrg $=$ RTrim $\$(\overline{O P}$ Txt $) \&$ ".Dat"

CPStrg $=$ RTrim $\$\left(\mathrm{CP}_{-}^{-} \mathrm{Txt}\right) \&$ ".Dat"

FNumA = FreeFile

Open IPStrg For Random Access Read As \#FNumA Len $=4$

FNumB $=$ FreeFile

Open OPStrg For Random Access Write As \#FNumB Len $=4$

End If

Select Case FunInProgress

Case TP_BRFT, TP_CMAG, TP_VABS, TP_VMXM, TP_VPAS CoFlag $=$ True

Case Else

CoFlag $=$ False

FileNum3 = FreeFile

Open CAStrg For Binary Access Read As \#FileNum3

If Not AutoMan Then

$\mathrm{FNumC}=$ FreeFile

Open CPStrg For Random Access Read As \#FNumC Len $=4$ End If

End Select

While NS < NS_Dat

For $\mathrm{J}=1$ To EData

Get \#FileNum1, , VarTemp

Varl = CVI(VarTemp)

Get \#FileNum1, , VarTemp

Var2 $=$ CVI (VarTemp)

Get \#FileNum1, , VarTemp

Var3 = CVI(VarTemp)

Datem $=\operatorname{Var} 3 * 2 \wedge 16+\operatorname{Var} 2 * 2 \wedge 8+\operatorname{Var} 1$

If Datem $<=1024$ Then

MX1 = Datem: MY1 = 0

Else

MX1 = Datem Mod 1024

MY1 = CInt $($ Datem $\backslash 1024)$

End If

If Not CoFlag Then

Get \#FileNum3, , Var'Temp

Varl $=$ CVI $($ VarTemp $)$ 
Get \#FileNum3, , VarTemp

Var2 = CVI(VarTemp)

Get \#FileNum3, , VarTemp

Var3 = CVI(VarTemp)

Cotem $=\operatorname{Var} 3 * 2 \wedge 16+\operatorname{Var} 2 * 2 \wedge 8+\operatorname{Var} 1$

If Cotem $<=1024$ Then

$\mathrm{MX} 2=$ Cotem: $\mathrm{MY} 2=0$

Else

MX2 $=$ Cotem Mod 1024

$\mathrm{MY} 2=\operatorname{CInt}(\operatorname{Cotem} \backslash 1024)$

End If

End If

If AutoMan Then

If Not CoFlag Then

CRTem $(J)=$ CRMX $(M X 2$, MY2 $)$

CYTem $(J)=$ CYMX $(M X 2, M Y 2)$

If CSFI $=1$ Then

$\operatorname{CRTem}(J)=$ Dat_Shift(CRTem $(J), 1)$

CYTem $(J)=$ Dat_Shift $($ CYTem $(J), 1)$

End If

End If

Else

MemPos $=$ Cotem $* 2+1$

If Not CoFlag Then

Get \#FNumC, MemPos, CRTem(J)

Get \#FNumC, MemPos + 1, CYTem(J)

If CSFI $=1$ Then

CRTem $(J)=$ Dat $\operatorname{Shift}(\mathrm{CRTem}(\mathrm{J}), 1)$

CYTem $(J)=$ Dat_Shift $($ CYTem $(J), 1)$

End If

End If

End If

If AutoMan Then

Select Case DF Dat

Case \&H1, \&H2, \&H3 'RQWA RQWC RQWB

$\operatorname{RTem}(J)=\mathrm{QRMX}(\mathrm{MX1}, \mathrm{MY} 1)$

$\mathrm{YTem}(J)=\mathrm{QYMX}(\mathrm{MX} 1, \mathrm{MY} 1)$

Case \&H5, \&H6, \&H7 'RAWQ RAWC RAWB

$\operatorname{RTem}(J)=\operatorname{ARMX}(M X 1, M Y 1)$

YTem $(J)=$ AYMX $(M X 1, M Y 1)$

Case \&H0, \&H4 'RBWA RBWQ

$\operatorname{RTem}(\mathrm{J})=\mathrm{BRMX}(\mathrm{MX} 1, \mathrm{MY} 1)$

YTem $(J)=$ BYMX $(M X 1$, MY1)

Case \&H8, \&H9, \&HA 'RCWA RCWB RCWQ

$\operatorname{RTem}(J)=\operatorname{CRTem}(J)$

End Select

$\operatorname{YTem}(\mathrm{J})=\operatorname{CYTem}(\mathrm{J})$

Else 


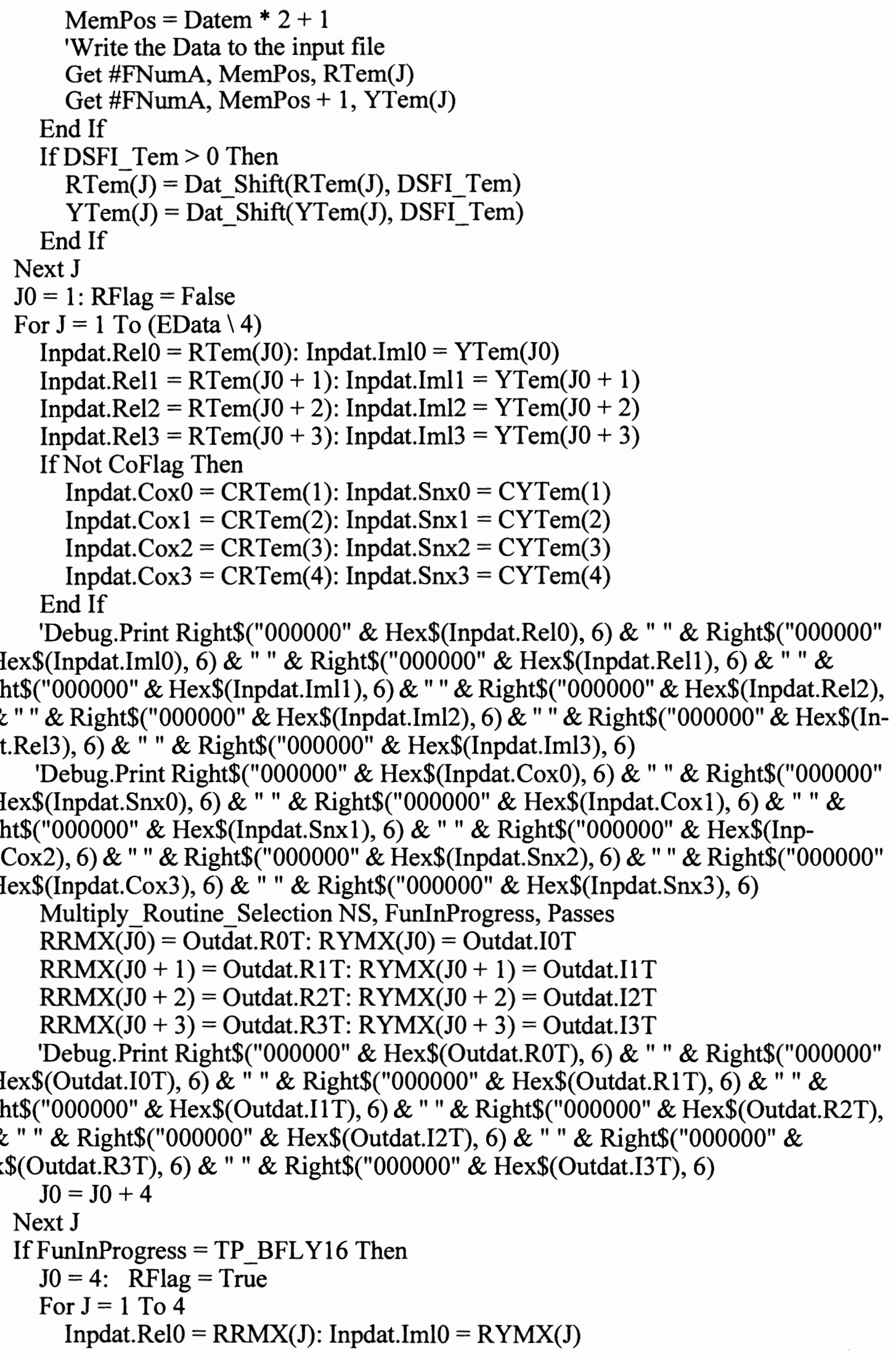




$$
\begin{aligned}
& \text { Inpdat.Rel1 }=\text { RRMX }(\mathrm{J}+4): \text { Inpdat.Iml1 }=\mathrm{RYMX}(\mathrm{J}+4) \\
& \text { Inpdat.Rel2 }=\mathrm{RRMX}(\mathrm{J}+8): \operatorname{Inpdat} \cdot \operatorname{Im} 12=\mathrm{RYMX}(\mathrm{J}+8) \\
& \text { Inpdat.Rel3 = RRMX }(\mathrm{J}+12) \text { : Inpdat.Iml3 = RYMX }(\mathrm{J}+12) \\
& \text { Inpdat. } \operatorname{Cox} 0=\mathrm{CRTem}(1) \text { : } \text { Inpdat.Snx0 }=\mathrm{CYTem}(1) \\
& \text { Inpdat.Cox1 }=\mathrm{CRTem}(\mathrm{J} 0+1) \text { : Inpdat.Snx1 }=\mathrm{CYTem}(\mathrm{J} 0+1) \\
& \text { Inpdat. } \operatorname{Cox} 2=\mathrm{CRTem}(\mathrm{J} 0+2) \text { : Inpdat.Snx2 }=\mathrm{CYTem}(\mathrm{J} 0+2) \\
& \text { Inpdat.Cox3 }=\mathrm{CRTem}(\mathrm{J} 0+3) \text { : Inpdat.Snx } 3=\mathrm{CYTem}(\mathrm{J} 0+3) \\
& \mathrm{J} 0=\mathrm{J} 0+3 \\
& \text { Multiply_Routine_Selection NS, FunInProgress, Passes } \\
& \operatorname{RRMX}(\bar{J})=\text { Outdat. R0T: } \operatorname{RYMX}(\mathrm{J})=\text { Outdat.I0T } \\
& \operatorname{RRMX}(\mathrm{J}+4)=\text { Outdat.R1T: } \operatorname{RYMX}(\mathrm{J}+4)=\text { Outdat.I1 T } \\
& \operatorname{RRMX}(\mathrm{J}+8)=\text { Outdat.R2T: } \mathrm{RYMX}(\mathrm{J}+8)=\text { Outdat.I } 2 \mathrm{~T} \\
& \operatorname{RRMX}(J+12)=\text { Outdat.R3T: RYMX }(J+12)=\text { Outdat.I3T }
\end{aligned}
$$

Next J

End If

For $\mathbf{J}=1$ To EData

Get \#FileNum2, , VarTemp

Var1 = CVI(VarTemp)

Get \#FileNum2, , VarTemp

$\operatorname{Var} 2=$ CVI(VarTemp)

Get \#FileNum2, , VarTemp

Var3 = CVI(VarTemp)

Datem $=\operatorname{Var} 3 * 2 \wedge 16+\operatorname{Var} 2 * 2 \wedge 8+\operatorname{Var} 1$

If Datem $<=1024$ Then

$$
\mathrm{MX1}=\text { Datem: } \mathrm{MY} 1=0
$$

Else

MX1 = Datem Mod 1024

MY1 = CInt $($ Datem $\backslash 1024)$

End If

If AutoMan Then

Select Case DF Dat

Case \&H4, \&H5, \&HA 'RBWQ RAWQ RCWQ $\mathrm{QRMX}(\mathrm{MX} 1, \mathrm{MY} 1)=\mathrm{RRMX}(\mathrm{J}): \mathrm{QYMX}(\mathrm{MX} 1, \mathrm{MY} 1)=\mathrm{RYMX}(\mathrm{J})$

Case \&H0, \&H1, \&H8 'RBWA RQWA RCWA ARMX $(M X 1, M Y 1)=R R M X(J): A Y M X(M X 1, M Y 1)=R Y M X(J)$

Case \&H3, \&H7, \&H9 'RQWB RAWB RCWB BRMX $(M X 1, M Y 1)=R R M X(J): B Y M X(M X 1, M Y 1)=R Y M X(J)$

Case \&H2, \&H6 'RAWC RQWC $\mathrm{CRMX}(\mathrm{MX} 1, \mathrm{MY} 1)=\mathrm{RRMX}(\mathrm{J}): \mathrm{CYMX}(\mathrm{MX} 1, \mathrm{MY} 1)=\mathrm{RYMX}(\mathrm{J})$

End Select

Else

MemPos $=$ Datem $* 2+1$

Put \#FNumB, MemPos, RRMX(J)

Put \#FNumB, MemPos + 1, RYMX(J)

End If

Next $\mathbf{J}$

$\mathrm{NS}=\mathrm{NS}+$ EData

If NS $=$ NS_Dat -4 Then 
$=\mathrm{TP}$ BRFIR Then rndFlag $=\overline{1}$

End If

Wend

Close

End Sub

Sub Save_Screen (Index1 As Integer)

On Error Resume Next

Dim LongName As String

Dim Title As String

Dim CDPath As String

If $($ Index1 $=$ MID_SAVE_AS) Or $($ ScreenInfo(ScrnStage $)$. Filename $=$ Untitled $)$ Then NeedToSave $=\overline{\text { False }}$

' Set default filter.

If TP TID $=$ TP_BLK Then

MainEditor.CMDialog_Edit.Filter $=$ "BDB Files $\left.\left({ }^{*}\right.$. bdb $)\right|^{*}$. BDB"

MainEditor.CMDialog_Edit.DefaultExt = "BDB"

Elself TP_TID $=$ TP_SYS Then

MainĒitor.CMDialog_Edit.Filter $=$ "SDB Files $\left.\left({ }^{*} . \mathrm{sdb}\right)\right|^{*}$. SDB"

MainEditor.CMDialog_Edit.DefaultExt $=$ "SDB"

End If

' Display Save As dialog.

MainEditor.CMDialog_Edit.FilterIndex $=1$

If Right(ScreenInfo(ScrnStage).FullPath, 1) $\diamond$ "l" Then

MainEditor.CMDialog_Edit.Filename $=$ ScreenInfo(ScrnStage).FullPath \& "।" \&

ScreenInfo(ScrnStage).Filename

End If

'If file has not been saved yet, set common dialog's

' default file name to *.dsp

If ScreenInfo(ScrnStage). Filename = "[Untitled]" Then

MainEditor.CMDialog_Edit.Filename = IIf(TP_TID = TP_BLK, "*.bdb", "*.sdb")

End If

MainEditor.CMDialog_Edit.Action $=2$

' Exit if user cancels the dialog.

If Err $=32755$ Then Exit Sub

Title $=$ MainEditor.CMDialog_Edit.Filetitle

LongName $=$ MainEditor.CMDialog_Edit.Filename

ScreenInfo(ScrnStage).FullPath = Left(LongName, Len(LongName) - Len(Title))

ScreenInfo(ScrnStage) .Filename $=$ MainEditor.CMDialog_Edit.Filetitle

' Adjust for root directory.

If Len(ScreenInfo(ScrnStage).FullPath) $=3$ Then

CDPath $=$ ScreenInfo(ScrnStage).FullPath

Else

CDPath $=$ Left(LongName, Len $($ LongName $)-$ Len(Title $)-1)$

End If

' Change directories for the editor and the viewer.

ChDir CDPath 
End If

MainEditor.MousePointer $=$ HOURGLASS

Write_Screen_To_File ScreenInfo(ScrnStage).FullPath, ScreenInfo(ScrnStage).Filename

MainEditor.MousePointer $=$ DEFAULT End Sub

Sub Scale_Data_Amount (DatSEL As Integer, IconID As Integer)

If DatSEL Then

BFPI_Tem $=$ BFPI_Dat Else

If IconID $=$ TP_MOVV Then DSFI_Tem $=0$ Else DSFI_Tem $=$ DSFI_Dat

Select Case IconID

Case TP_BFLY16

DSFI Tem $=$ AutoDSFI

Case TP_BFLY4, TP_BWND4

DSFI Tem $=$ AutoD SFI -2

Case TP_BFLY2, TP_BWND2, TP_BFCT, TP_BFCT2

DSFI_Tem $=$ AutoDSFI -3

Case TP_CMAG

DSFI Tem $=$ AutoDSFI -4

Case Else

End Select

DSFI_Tem $=0$

If DSFI Tem $<0$ Then DSFI_Tem $=0$

End If

BFPI Tem $=$ BFPO Dat

End Sub

Sub ScrollBar_Positioning (InstVS As Control, InstHS As Control, Wks As Control, RootWks As Control)

InstHS. Top $=$ RootWks. Height

InstHS.Left $=0$

InstHS. Width $=$ RootWks. Width

InstHS.Max $=$ Wks.ScaleWidth - RootWks.ScaleWidth

If (RootWks.ScaleWidth $<$ Wks.ScaleWidth) Then

InstHS.Enabled $=$ True

Diff $=$ Wks.ScaleWidth $\backslash$ RootWks.ScaleWidth

InstHS.LargeChange $=$ InstHS.Max $\backslash$ Diff

Else

InstHS.Enabled $=$ False

End If

InstHS.Refresh

InstVS.Top $=0$

InstVS.Left $=$ RootWks. Width

InstVS. Height $=$ RootWks. Height

InstVS.Max = Wks. ScaleHeight - RootWks.ScaleHeight

If (RootWks.ScaleHeight < Wks. ScaleHeight) Then 


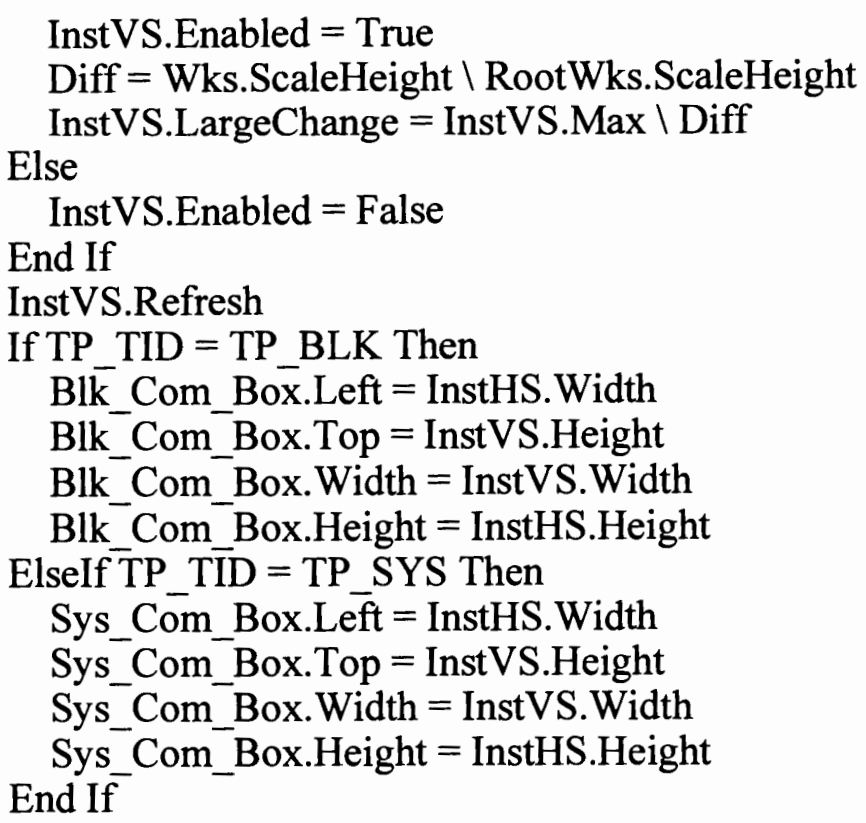

\section{End Sub}

'When the MousePointer is within the Editing area, the MousePointer 'is changed depending on the Tool being used. This routine is called 'from the MouseMove event for the Sys and Block Work_Space to change the 'MousePointer.

Sub Set_MousePointer ()

Select Case CurrentTool

Case TP_SELECT

Sys_W WorkSpace. MousePointer $=$ ARROW

Case TP LINE

Sys_W WorkSpace.MousePointer $=$ ARROW

Case TP TEXT

Sys $\overline{\text { WorkSpace. }}$ MousePointer = I BEAM

Case Else

Sys_WorkSpace.MousePointer $=$ DEFAULT

End Select

End Sub

Sub Start_System_Click ()

Dim DSPDrive As String, DSPDir As String

Dim TemVal As Integer

CurPath $\$=$ CurDir\$

Stop_System.Enabled $=$ True

Start_System.Enabled $=$ False

Select Case TP TID

Case TP SYS

If Pic_SysWork.Visible Then Prepare_New_Sys_Icon XSI(SICount), YSI(SICount) If SISiz $>0$ And LCount $>0$ Then 
AutoDSFI $=0: \mathrm{I}=1$

Screen. MousePointer $=11$

While I $<=$ ArrayCount

If FuncCode $(\mathrm{I}, 0)>=\& \mathrm{H} 0$ And FuncCode $(\mathrm{I}, 0)<=\& \mathrm{H} 1 \mathrm{E}$ Then

LookUp_Func_ID Pic_Work, FuncCode $(\mathrm{I}, 0)$

THISFN_SECT $=$ Pic_Work.Tag \& " Pass" \& Format\$(Stage(I))

Get Ini File Settings

Create Port Array NS Dat, DF Dat

Scale_Data_Amount DSFISEL, FuncCode $(I, 0)$

Process_IO_Data FuncCode(I, 0), Stage(I)

DSFO $\bar{D}$ at $=$ AutoDSFI

BFPO_Dat $=$ DSFI_Tem + BFPI_Tem

KEY STR = Format $\$$ (DSFO Dat) \& "," \& Format\$(BFPO Dat)

THISFN_INI)

$\mathrm{R}=$ WritePrivateProfileString(T) $\overline{\mathrm{T} H I S F N}$ _SECT, "DSFO BFPO", KEY_STR,

End If

$\mathrm{I}=\mathrm{I}+1$

Wend

Screen.MousePointer $=0$

'Unload StatusDlg

Else execution"

MsgBox "No function icons selected or No Line path connection", 16, "Error in

Start_System.Enabled $=$ True

Stop_System. Enabled $=$ False

End If

Initiate SignEdit

Case TP BLK

If Pic_Work.Visible Then Prepare_New_Icon XArr(ArraySiz), YArr(ArraySiz)

If ArrayCount $>0$ And LineCount $>0$ Then

'StatusDlg.Show Modeless

AutoDSFI $=0: \mathrm{I}=1$

Screen.MousePointer $=11$

While $\mathrm{I}<=$ ArrayCount

If FuncCode $(\mathrm{I}, 0)>=\& \mathrm{H} 0$ And FuncCode $(\mathrm{I}, 0)<=\& \mathrm{H} 1 \mathrm{E}$ Then

LookUp_Func ID Pic Work, FuncCode $(1,0)$

THISFN_SECT $=$ Pic_Work.Tag \& " Pass" \& Format $\$($ Stage $(\mathrm{I}))$

Get_Ini_File_Settings

Create_Port_Array NS_Dat, DF_Dat

Scale_Data_Amount DSFISEL, FuncCode $(I, 0)$

Process IO Data FuncCode(I, 0), Stage(I)

DSFO $\bar{D}$ at $=$ AutoDSFI

BFPO_Dat $=$ DSFI_Tem + BFPI Tem

KEY_STR $=$ Format $\$$ (DSFO_Dat) \& "," \& Format\$(BFPO_Dat)

THISFN_INI)

$\mathrm{R}=$ WritePrivateProfileString(T) $\overline{\mathrm{T} H I S F N}$ _SECT, "DSFO BFPO", KEY_STR,

End If

$\mathrm{I}=\mathrm{I}+1$ 


\section{Wend}

Screen. MousePointer $=0$

Scriptitnow $=$ True

Else execution"

'Unload StatusDig

MsgBox "No function icons selected or No Line path connection", 16, "Error in

Start System. Enabled $=$ True

Stop_System.Enabled $=$ False

End If

End Select

Initiate_SignEdit

Start_System. Enabled $=$ True

End $\mathrm{Su} \overline{\mathrm{b}}$

Sub Stop_System_Click ()

Start System. Enabled $=$ True

Stop_System. Enabled $=$ False

End Sub

Sub STxtEdit_Change (Index As Integer)

TxtUnFocus Index

STxtEdit(Index $) \cdot$ Height $=$ TextHeight $($ STxtEdit(Index $))+4$

STxtEdit(Index).Width $=$ TextWidth(STxtEdit(Index)) $* 1.4+20$

If STxtEdit(Index).Text $\diamond$ "" Then TxtFocus Index

STxtEnable $=$ False

End Sub

Sub STxtEdit_GotFocus (Index As Integer)

TxtUnFocus STxtSelected

If STxtEdit(STxtSelected).Text $=$ "" Then

If Index $<$ STxtNum - 1 Then STxtEnable = False

If Not STxtEnable Then STxtEdit(STxtSelected).Visible $=$ False

End If

STxtSelected $=$ Index

TxtFocus Index

End Sub

Sub STxtEdit_MouseDown (Index As Integer, Button As Integer, Shift As Integer, X As Single, Y As Single)

If CurrentTool $\diamond$ TP_TEXT Then

STxtEdit(Index).Drag 1

DragX $=X / 15$

DragY $=\mathrm{Y} / 15$

Sys_WorkSpace.SetFocus

End If

'TxtUnFocus STxtSelected

End Sub 
Sub STxtEdit_MouseMove (Index As Integer, Button As Integer, Shift As Integer, X As Single, Y As Single)

STxtEdit(Index).MousePointer $=$ IIf(CurrentTool $=$ TP_SELECT, ARROW, DEFAULT)

End Sub

Sub STxtEdit_MouseUp (Index As Integer, Button As Integer, Shift As Integer, X As Single, Y As Single)

If CurrentTool $\diamond$ TP_TEXT Then STxtEdit(Index).Drag 2

End Sub

Sub Sys_InstHScroll_Changing ()

Sys_WorkSpace.Left $=-$ Sys_InstHScroll.Value

End Sub

Sub Sys_InstHScroll_GotFocus ()

If Pic_SysWork.Visible Then

'If Not SysToolFlag Then Prepare_New_Sys_Icon XSI(SICount), YSI(SICount)

'Tool_Select. Value $=$ False

'CurrentTool $=\&$ HEE

'SysToolFlag $=$ True

End If

End Sub

Sub Sys_InstVScroll_Changing ()

Sys_WorkSpace. Top $=-$ Sys_InstVScroll.Value

End $\mathrm{Su} \bar{b}$

Sub Sys_InstVScroll_GotFocus ()

If Pic_SysWork. Visible Then

'If Not SysToolFlag Then Prepare_New_Sys_Icon XSI(SICount), YSI(SICount)

'Tool_Select.Value $=$ False

'CurrentTool $=$ \&HEE

'SysToolFlag $=$ False

End If

End Sub

Sub Sys_System_Click ()

'If Not Tool_Select.Value Then Tool_Select.Value $=$ True

If TP TID $=$ TP BLK Then

'If the Block ToolPalette Extension is visible, hide it

If ToolPalette_BlkExt.Visible Then ToolPalette_BlkExt.Visible $=$ False

If Not SystemEditor.Visible Then SystemEditor. Visible $=$ True

SystemEditor. WindowState $=$ MAXIMIZED

ElseIf TP_TID $=$ TP_SYS Then

If Not BlockEditor. Visible Then BlockEditor.Visible $=$ True

BlockEditor. WindowState $=$ MAXIMIZED 
End If

End Sub

Sub Sys_WorkSpace_DragDrop (Source As Control, X As Single, Y As Single)

Dim Movable As Integer

$\mathrm{XTArr}=\mathrm{X}-$ DragX: $\mathrm{YTArr}=\mathrm{Y}-$ DragY

If TypeOf Source Is TextBox Then

TxtUnFocus STxtSelected

Source.Move XTArr, YTArr

TxtFocus STxtSelected

Elself TypeOf Source Is PictureBox Then

Movable $=$ True

SavedMode $=$ Sys_WorkSpace. . DrawMode

'Check to see there is line between the icon to be moved and if it does, erase it 'and update the current line position of the move icon.

For I = 0 To LCount -1

If YPts $(I, 0)=$ YSI $($ SICount $)+16$ And XPts $(I, 0)=X S I($ SICount $)-2$ Then

If XTArr $-2<=$ XPts $(\mathrm{I}, 1)$ Then

Msg = "Do not drag active icon beyond the left icon"

MsgBox Msg, 16, "Dragging Icon Error"

Movable $=$ False

Exit For

End If

ElseIf YPts $(I, 1)=$ YSI $($ SICount $)+8$ And XPts $(I, 1)=X S I($ SICount $)+32$ Then

If XTArr $+32>=X P t s(I, 0)$ Then

Msg = "Do not drag active icon beyond the left icon"

MsgBox Msg, 16, "Dragging Icon Error"

Movable $=$ False

Exit For

End If

ElseIf YPts $(I, 1)=$ YSI (SICount $)+24$ And XPts $(I, 1)=X S I($ SICount $)+32$ Then

If XTArr $+32>=X P t s(I, 0)$ Then

Msg = "Do not drag active icon beyond the left icon"

MsgBox Msg, 16, "Dragging Icon Error"

Movable $=$ False

Exit For

End If

Elself YPts $(I, 1)=$ YSI(SICount $)+16$ And XPts $(I, 1)=X S I($ SICount $)+32$ Then

If XTArr $+32>=X P t s(I, 0)$ Then

Msg = "Do not drag active icon beyond the left icon"

MsgBox Msg, 16, "Dragging Icon Error"

Movable $=$ False

Exit For

End If

End If

Next I

If Movable Then

For $I=0$ To LCount -1 
If YPts(I, 0) = YSI(SICount $)+16$ And XPts(I, 0) $=$ XSI(SICount $)-2$ Then

'Erase the Old Line

XFinish $=$ XPts $(I, 0):$ YFinish $=$ YPts $(I, 0)$

XStart $=$ XPts(I, 1): YStart $=$ YPts $(I, 1)$

Sys_WorkSpace.DrawMode $=$ NOT_COPY_PEN

Draw_Icon_Line XStart, YStart, XFinish, YFinish

Sys_WorkSpace. DrawMode $=$ SavedMode

'Update the new position line

$\mathrm{XPts}(\mathrm{I}, 0)=\mathrm{XTArr}-2: \mathrm{YPts}(\mathrm{I}, 0)=$ YTArr +16

$\mathrm{XFinish}=\mathrm{XTArr}-2:$ YFinish $=$ YTArr +16

Draw_Icon_Line XStart, YStart, XFinish, YFinish

ElseIf YPts $(\mathrm{I}, \overline{1})=$ YSI(SICount) +8 And XPts(I, 1) $=$ XSI(SICount $)+32$ Then

'Erase the Old Line

$\mathrm{XFinish}=\mathrm{XPts}(\mathrm{I}, 0):$ YFinish $=$ YPts $(\mathrm{I}, 0)$

XStart $=$ XPts(I, 1): YStart $=$ YPts $(I, 1)$

Sys_WorkSpace.DrawMode $=$ NOT_COPY_PEN

Draw Icon Line XStart, YStart, XFinish, YFinish

Sys_WorkSpace. DrawMode $=$ SavedMode

'Update the new position line

$\mathrm{XPts}(\mathrm{I}, 1)=\mathrm{XTArr}+32: \mathrm{YPts}(\mathrm{I}, 1)=\mathrm{YTArr}+8$

XStart $=$ XTArr $+32:$ YStart $=$ YTArr +8

Draw Icon_Line XStart, YStart, XFinish, YFinish

ElseIf YPts $(\mathrm{I}, \overline{1})=$ YSI(SICount $)+24$ And XPts $(I, 1)=X S I($ SICount $)+32$ Then

'Erase the Old Line

XFinish $=X P t s(I, 0):$ YFinish $=$ YPts $(I, 0)$

XStart $=$ XPts $(I, 1):$ YStart $=$ YPts $(I, 1)$

Sys_WorkSpace.DrawMode $=$ NOT_COPY_PEN

Draw_Icon_Line XStart, YStart, XFinish, YFinish

Sys_W WorkSpace. .DrawMode $=$ SavedMode

'Update the new position line

$\mathrm{XPts}(\mathrm{I}, 1)=\mathrm{XTArr}+32: \mathrm{YPts}(\mathrm{I}, 1)=\mathrm{YTArr}+24$

XStart $=$ XTArr $+32:$ YStart $=$ YTArr +24

Draw_Icon_Line XStart, YStart, XFinish, YFinish

ElseIf YPts $(I, \overline{1})=$ YSI(SICount $)+16$ And XPts $(I, 1)=$ XSI $($ SICount $)+32$ Then

'Erase the Old Line

$\mathrm{XFinish}=\mathrm{XPts}(\mathrm{I}, 0):$ YFinish $=$ YPts $(\mathrm{I}, 0)$

XStart $=$ XPts(I, 1): YStart $=$ YPts(I, 1)

Sys_WorkSpace.DrawMode $=$ NOT_COPY_PEN

Draw_Icon_Line XStart, YStart, XFinish, YFinish

Sys_WorkSpace. .DrawMode $=$ SavedMode

'Update the new position line

$\mathrm{XPts}(\mathrm{I}, 1)=\mathrm{XTArr}+32: \mathrm{YPts}(\mathrm{I}, 1)=\mathrm{YTArr}+16$

XStart $=$ XTArr $+32:$ YStart $=$ YTArr +16

Draw_Icon_Line XStart, YStart, XFinish, YFinish

End If

Next I

XSI(SICount $)=$ XTArr

YSI(SICount $)=$ YTArr 
Source.Move XTArr, YTArr

End If

End If

End Sub

Sub Sys_WorkSpace_Load 0

Sys_WorkSpace.Top $=0$

Sys_WorkSpace.Left $=0$

Sys_WorkSpace. BorderStyle $=$ NONE

Pic_SysWork.BorderStyle $=$ NONE

Pic_SysWork.Move 0, 0, 34, 54

End Sub

Sub Sys_WorkSpace_MouseDown (Button As Integer, Shift As Integer, X As Single, Y As Single)

If Button $=$ LEFT_BUTTON And TP_TID $=$ TP_SYS Then

XOld $=$ XSI(SIC

Select Case CurrentTool

Case TP_TEXT

If STxtNum $>0$ Then

If STxtEnable And STxtEdit(STxtNum - 1).Text = "' Then

TxtUnFocus STxtNum - 1

STxtEdit(STxtNum - 1).Move X, Y

STxtEdit(STxtNum - 1).SetFocus

End If

End If

If Not STxtEnable Then

If STxtNum > 1 Then Load STxtEdit(STxtNum)

STxtEdit(STxtNum).Top $=$ Y

STxtEdit(STxtNum).Left $=$ X

STxtEdit(STxtNum).Visible $=$ True

STxtEdit(STxtNum).Text = ""

STxtEdit(STxtNum).SetFocus

STxtNum $=$ STxtNum +1

STxtEnable $=$ True

End If

Case TP SELECT

TxtUnFocus STxtSelected

For I = 1 To SISiz $>=\mathrm{Y})$ Then

If $(\mathrm{XSI}(\mathrm{I})<=\mathrm{X}$ And $(\mathrm{XSI}(\mathrm{I})+34)>=\mathrm{X})$ And $(\mathrm{YSI}(\mathrm{I})<=\mathrm{Y}$ And $(\mathrm{YSI}(\mathrm{I})+54)$

\footnotetext{
UnRFocus_Control MainEditor.Pic_SysWork

$\mathrm{R}=$ BitBlt(Sys_WorkSpace.hDC, XŌld, YOld, 34, 54, Pic_SysWork.hDC, 0,0, SRCCOPY)

Pic_SysWork.Picture $=$ LoadPicture()

Pic_SysWork.Move XSI(I), YSI(I)

Get_Function_Pic Pic_SysWork, FuncCode(I, 1)

$\mathrm{R}=$ PatBlt(Sys_WorkSpace.hDC, XSI(I), YSI(I), 34, 54, WHITENESS)
} 
Pic_Box_Paint Pic_SysWork, Pass(I), FuncCode(I, 1)

SICount $=$ I

Exit Sub

End If

Next I

Case TP_LINE

For I $=1$ To SISiz

$>=Y)$ Then

If $(\mathrm{XSI}(\mathrm{I})<=\mathrm{X}$ And $(\mathrm{XSI}(\mathrm{I})+34)>=\mathrm{X})$ And $(\mathrm{YSI}(\mathrm{I})<=\mathrm{Y}$ And $(\mathrm{YSI}(\mathrm{I})+54)$

'Check to see which icons is selected so that Icon's ID can be properly set

Select Case StartEvent

Case False

IniDraw $=$ True

StartEvent $=$ True

'Store the initial start of the line to draw

StartX $=$ XSI $(\mathrm{I})+16$

Start $Y=$ YSI $(I)+16$

'Make the last location equal the starting location

OldX $=$ Start $X$

OldY $=$ Start $Y$

DsplyFlg1 = FuncCode $(\mathrm{I}, 1)$

ArrayFlg1 = I

XPts $($ LCount, 1$)=$ XSI $(I)+32:$ YPts $($ LCount, 1$)=$ YSI $(I)+16$

Case True

StartEvent $=$ False

FinDraw $=$ True

DsplyFlg2 $=$ FuncCode $(\mathrm{I}, 1)$

ArrayFlg2 = I

End Select

$\mathrm{XPts}(\mathrm{LCount}, 0)=\mathrm{XSI}(\mathrm{I})-2: \operatorname{YPts}($ LCount, 0$)=\mathrm{YSI}(\mathrm{I})+16$

Exit Sub

End If

Next I

Msg = "Invalid -- No Icon selected"

MsgBox Msg, 16, "Line Editor"

FinDraw $=$ False: StartEvent $=$ False

$\mathrm{XPts}($ LCount, 0$)=0: \mathrm{YPts}($ LCount, 0$)=0$

$\mathrm{XPts}($ LCount, 1$)=0: \mathrm{YPts}(\operatorname{LCount}, 1)=0$

If IniDraw Then

DrawLine Sys_WorkSpace, StartX, StartY, OldX, OldY

IniDraw $=$ False

End If

Case TP_UNLINE

For $\mathrm{I}=1$ To SISiz

$>=\mathrm{Y})$ Then

If $(\mathrm{XSI}(\mathrm{I})<=\mathrm{X}$ And $(\mathrm{XSI}(\mathrm{I})+34)>=\mathrm{X})$ And $(\mathrm{YSI}(\mathrm{I})<=\mathrm{Y}$ And $(\mathrm{YSI}(\mathrm{I})+54)$

Select Case StartEvent

Case False 
IniDraw $=$ True

StartEvent $=$ True

$\mathrm{StartX}=\mathrm{XSI}(\mathrm{I})+16$

StartY $=$ YSI $(I)+16$

OldX $=$ StartX

OldY $=$ Start $Y$

DsplyFlg1 $=$ FuncCode $(\mathrm{I}, 1)$

ArrayFlg1 $=$ I

DisLineX1 $=$ XSI $(\mathrm{I})+32$ : DisLineY1 $=$ YSI $(\mathrm{I})+16$

Sys_WorkSpace. DrawStyle $=$ DASH

Case True

StartEvent $=$ False

FinDraw $=$ True

DsplyFlg2 $=$ FuncCode $(\mathrm{I}, 1)$

ArrayFlg2 = I

End Select

DisLineX2 $=$ XSI(I) $-2:$ DisLineY2 $=$ YSI $(\mathrm{I})+16$

Exit Sub

End If

Next I

Msg = "Invalid -- No Icon selected"

MsgBox Msg, 16, "UnLine Editor"

IniDraw $=$ False: FinDraw $=$ False

DisLineX1 $=0$ : DisLineY1 $=0$

DisLineX2 $=0$ : DisLineY2 $=0$

If IniDraw Then

DrawLine Sys_WorkSpace, StartX, StartY, OldX, OldY

IniDraw $=$ False

End If

End Select

End If

End Sub

Sub Sys_WorkSpace_MouseMove (Button As Integer, Shift As Integer, X As Single, Y As Single)

'Caculate pixel mouse is currently over

$\mathrm{Xpix}=\mathrm{X}$

$\mathrm{YPix}=\mathrm{Y}$

'The Mouse coordinates are always displayed within the StatusArea

Display_Mouse_Coordinates Xpix, YPix

'Depending on the Current tool, and in the case of the select tool, 'the state of the selection, the MousePointer may change

If StartEvent Then

DrawLine Sys_WorkSpace, StartX, StartY, OldX, OldY

DrawLine Sys_WorkSpace, StartX, StartY, Xpix, YPix

OldX $=X$

OldY $=$ Y

End If 
End Sub

Sub Sys_WorkSpace_MouseUp (Button As Integer, Shift As Integer, X As Single, Y As Single)

If Button = LEFT_BUTTON And TP_TID $=$ TP_SYS Then

Select Case CurrentTool

Case TP LINE

If Not StartEvent And FinDraw Then

'Erase the previous line

DrawLine Sys_WorkSpace, StartX, StartY, OldX, OldY

OldX1 $=$ XPts(LCount, 0$)$

OldY1 $=$ YPts $($ LCount, 0$)$

StartX $=$ StartX +16

'Check for invalid operation

If StartX > OldX1 Then

to each other"

Msg = "Click data line from left to right Or" \& CRLF \& "Icons are too close

GoTo SysLine_Err

Elself DsplyFlg1 = TP_DISPLAY And StartX $<$ OldX Then

Msg = "Invalid Operation -- Display icon should be clicked last"

GoTo SysLine Err

ElseIf DsplyFlg1 $=$ TP_SIGNAL Or DsplyFlg1 $=$ TP_DISPLAY Or DsplyFlg1

$=\mathrm{TP}$ AIME Then

$=$ TP_AIME Then

If DsplyFlg2 = TP_DISPLAY Or DsplyFlg2 $=$ TP_SIGNAL Or DsplyFlg2

Msg = "Invalid Operation"

GoTo SysLine_Err

End If

ElseIf DsplyFlg2 = TP_SIGNAL Then

Msg = "Invalid Operation -- Input to Signal icon is unavailable"

End If

GoTo SysLine_Err

Ypts(LCount, 1)

'Swap_Values Xpts(LCount, 0), Xpts(LCount, 1), Ypts(LCount, 0),

'StartX $=$ Xpts(LCount, 1)

'StartY = Ypts(LCount, 1)

'OldX1 = Xpts $($ LCount, 0$)$

'OldY1 = Ypts(LCount, 0$)$

If DsplyFlg2 = TP DISPLAY Then

YPts $($ LCount, 1$)=$ YPts $($ LCount, 1$)-8$

ElseIf DsplyFlg1 $=$ TP_AGMEM Then

YPts $($ LCount, 1$)=\overline{Y P t s}($ LCount, 1$)+8$

End If

'Draw new final line

Start Y = YPts(LCount, 1)

Draw_Icon_Line StartX, StartY, OldX1, OldY1

$\mathrm{OldX}=\mathrm{Ol} \overline{\mathrm{dX}} 1$

OldY $=$ OldY 1 
'The left side is AG and the right side is AIME, then the array of AG 'contained the general index of AIME, not the identify index of AIME If DsplyFlg1 = TP_AGMEM And DsplyFlg2 = TP_AIME Then AGMPort(ArrayFlg1, 1) = ArrayFlg2 'input value of

ElseIf DsplyFlg1 = TP_AIME And DsplyFlg2 = TP_AGMEM Then AGMPort(ArrayFlg $\overline{2}, 0$ ) $=$ ArrayFlg1

ElseIf DsplyFlg1 = TP_AGMEM And DsplyFlg2 $=$ TP_DISPLAY Then DisplayPort (ArrayFlg 2 ) $=$ ArrayFlg1

End If

FinDraw $=$ False: IniDraw $=$ False

LCount $=$ LCount +1

End If

Case TP_UNLINE

If Not StartEvent And FinDraw Then

'Erase the previous line

DrawLine Sys_WorkSpace, StartX, StartY, OldX, OldY

Sys_WorkSpace.DrawStyle $=$ SOLID

ChangePosition $=$ False

For I $=0$ To LCount -1

If XPts $(\mathrm{I}, 0)=$ DisLineX2 And XPts(I, 1) $=$ DisLineX1 Then ChangePosition

$=$ True

If ChangePosition Then

$$
\begin{aligned}
& \mathrm{XPts}(\mathrm{I}, 0)=\mathrm{XPts}(\mathrm{I}+1,0): \operatorname{YPts}(\mathrm{I}, 0)=\mathrm{YPts}(\mathrm{I}+1,0) \\
& \mathrm{XPts}(\mathrm{I}, 1)=\mathrm{XPts}(\mathrm{I}+1,1): \operatorname{YPts}(\mathrm{I}, 1)=\mathrm{YPts}(\mathrm{I}+1,1)
\end{aligned}
$$

End If

Next I

If ChangePosition Then

If DsplyFlg1 $=$ TP_DISPLAY Or DsplyFlg2 $=$ TP_DISPLAY Then

DisLineY1 $=$ DisLineY1 -8

ElseIf DsplyFlg1 = TP_AGMEM Then

DisLine $\mathrm{Y} 1=$ DisLine $\mathrm{Y} 1+8$

End If

Sys_WorkSpace.DrawMode $=$ WHITENESS_PEN

Draw_Icon_Line DisLineX1, DisLineY1, DisLineX2, DisLineY2

Sys_WorkSpace.DrawMode $=$ COPY_PEN

If LCount $>0$ Then LCount $=$ LCount -1 Else LCount $=0$

ChangePosition $=$ False

If DsplyFlg1 $=$ TP_AGMEM And DsplyFlg2 $=$ TP_AIME Then AGMPort(ArrayFlg1, 1) $=0$

ElseIf DsplyFlg1 = TP_AIME And DsplyFlg2 $=$ TP_AGMEM Then AGMPort(ArrayFlg $\overline{2}, 0)=0$

End If

Else

Msg = "Invalid Operation Or No Line TO delete" \& CRLF \& "Select icon from left to right"

MsgBox Msg, 16, "UnLine Editor"

DisLineX1 $=0$ : DisLineY1 $=0$

DisLineX2 $=0$ : DisLineY2 $=0$ 
End If

End If

FinDraw $=$ False: IniDraw $=$ False

End Select

End If

Exit Sub

SysLine_Err:

MsgBox Msg, 16, "Line Editor Error"

XPts $($ LCount, 0$)=0:$ YPts $($ LCount, 0$)=0$

XPts $($ LCount, 1) $=0:$ YPts $($ LCount, 1) $=0$

FinDraw $=$ False: IniDraw $=$ False

End Sub

Sub SystemEditor_Close ()

SystemEditor. Visible $=$ False

End Sub

Sub SystemEditor_Resize ()

If LastSysWinState = MAXIMIZED Then

TP_TID = TP_MAIN

ScrnStage $=0$

If Pic_SysWork.Visible Then Prepare_New_Sys_Icon XSI(SICount), YSI(SICount)

If ToolPalette_BlkExt.Visible Then ToolPalette_BlkExt.Visible $=$ False

ToolPalette_Icons_Initialize

ElseIf SystemEditor. WindowState = MAXIMIZED Then

Sys_Root_WkSpace.Move 0, 0, SystemEditor.ClientWidth - Sys_InstVScroll.Width, SystemEditor.ClientHeight - Sys_InstHScroll.Height

TP_TID = TP_SYS

ScrnStage $=2$

ToolPalette_Icons_Initialize

Sys_System.Caption = "Block"

ScrollBar_Positioning Sys_InstVScroll, Sys_InstHScroll, Sys_WorkSpace,

Sys_Root_WkSpace

PFlag $=$ Stage_Flag

Pan_NSys.Caption $=$ Format $\$($ PFlag $)$

Pan_NSys. Visible $=$ True

Pan_NSysTxt. Visible $=$ True

If Not Tool_Select.Value Then Tool_Select.Value = True Else Initiate_SignEdit

If NeedToSave Then Menu_FileSelection_Click MID_SAVE_AS

End If

LastSys WinState $=$ SystemEditor. WindowState

End Sub

'If the button is depressed the pic_work contained the selected picture

Sub Tool_Blk1_Click ()

ArrayCount $=$ ArrayCount +1

Xipos $=0+$ Abs(Blk_WorkSpace.Left $)$ 


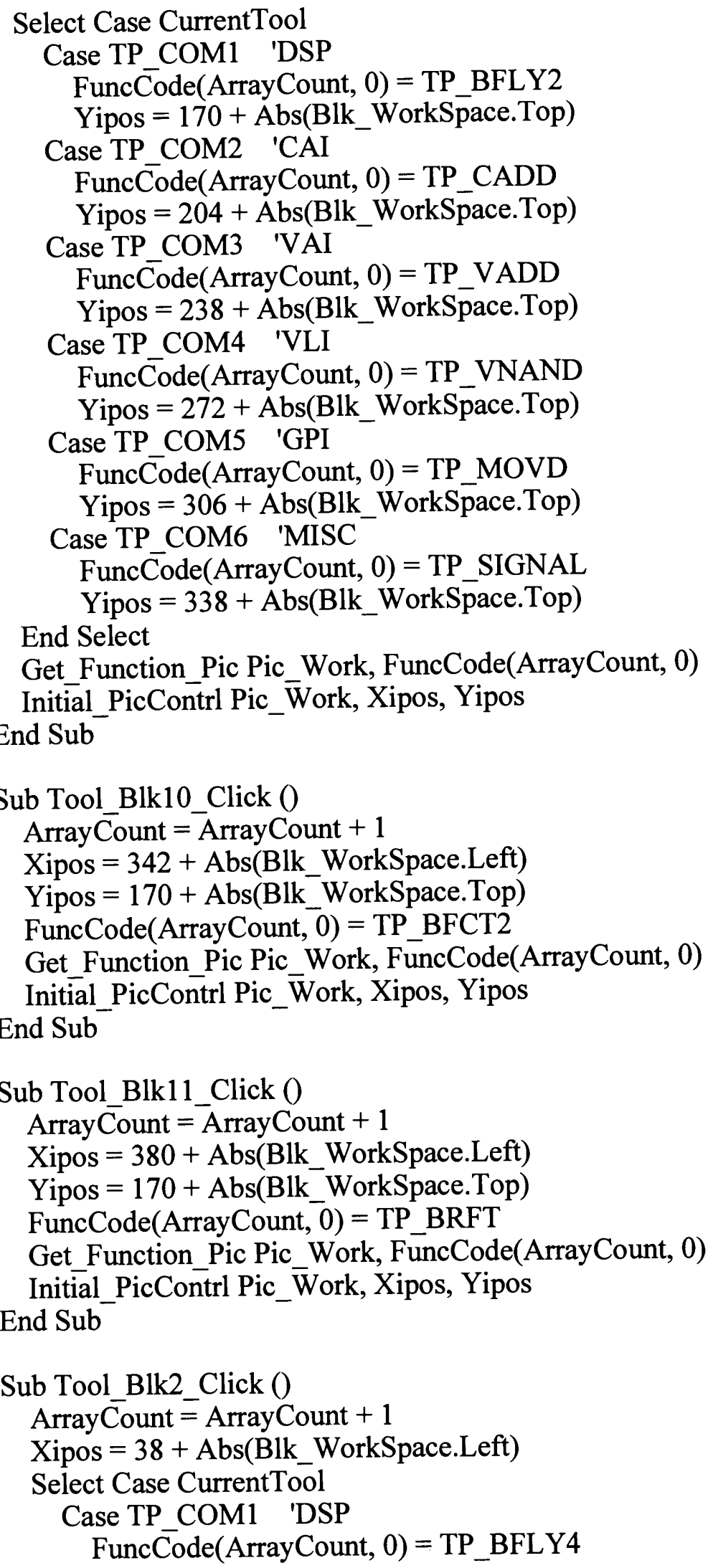




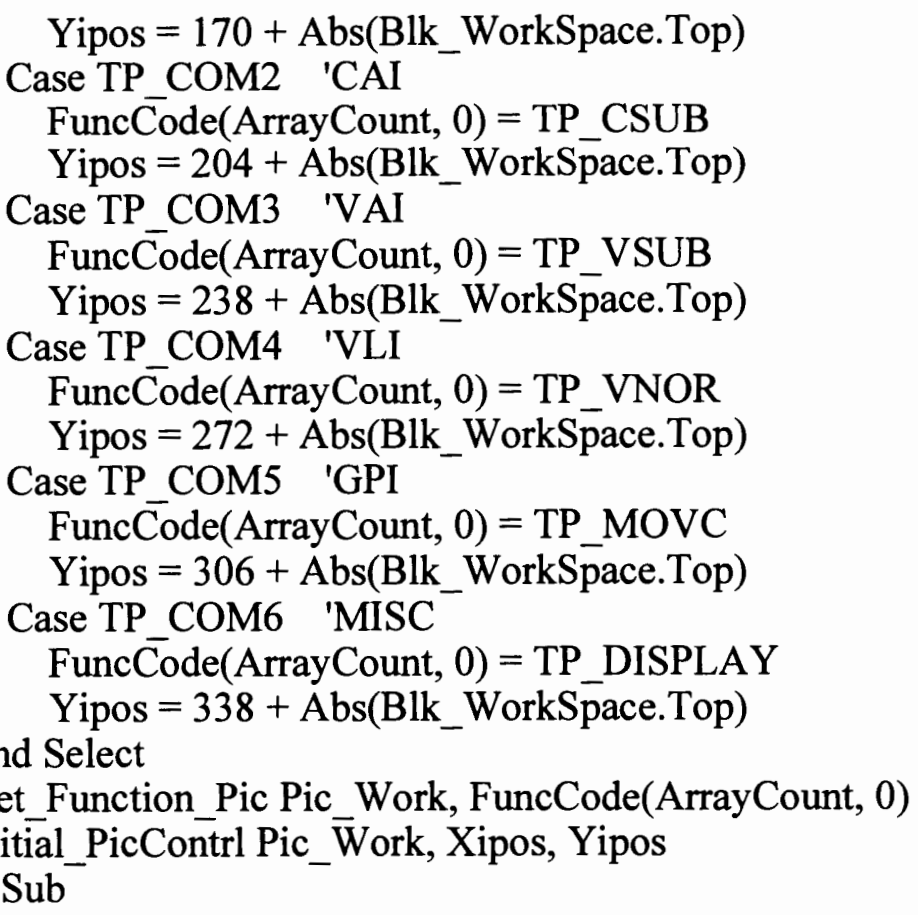

End Sub

Sub Tool_Blk3_Click ()

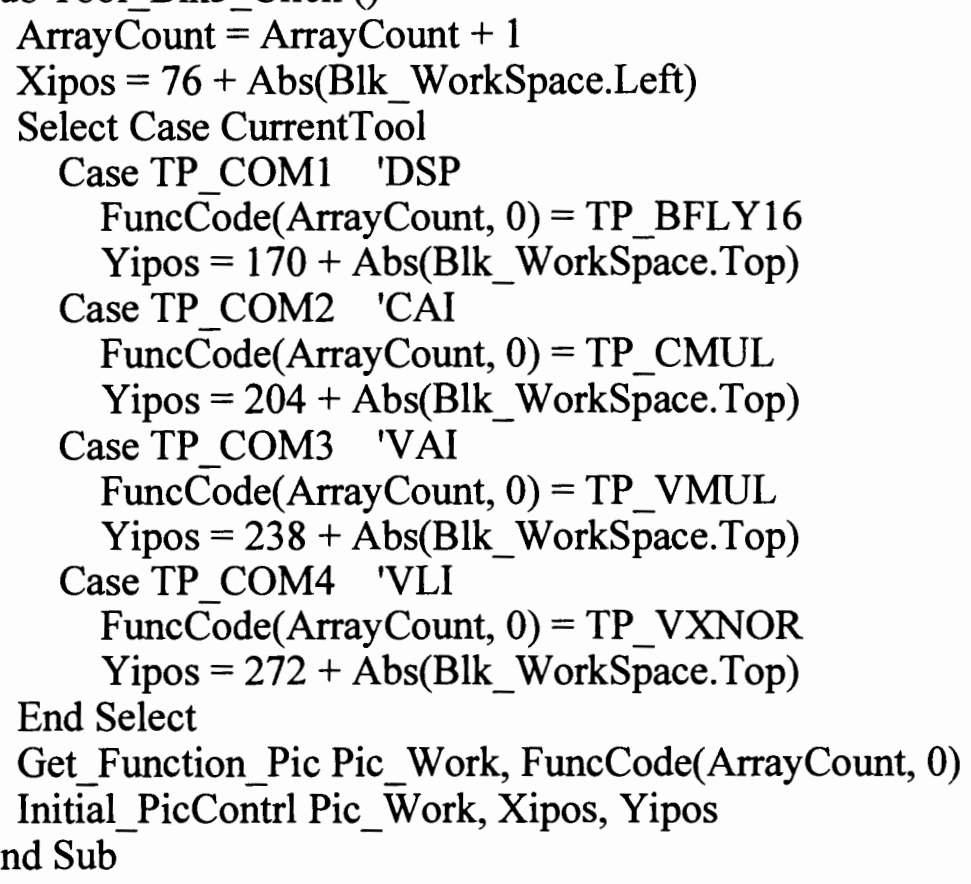

Sub Tool_Blk4_Click ()

ArrayCount $=$ ArrayCount +1

Xipos $=114+$ Abs(Blk_WorkSpace.Left)

Select Case CurrentTool

Case TP_COM1 'DSP

FuncCode $($ ArrayCount, 0$)=$ TP_BWND2 
Yipos $=170+$ Abs(Blk_WorkSpace.Top)

Case TP_COM2 'CAI

FuncCode(ArrayCount, 0) $=$ TP_CMAG

Yipos $=204+$ Abs(Blk_WorkSpace.Top)

Case TP_COM3 'VAI

FuncCode(ArrayCount, 0) $=$ TP_VABS

Yipos $=238+$ Abs(Blk_WorkSpace.Top)

Case TP_COM4 'VLI

Func Code(ArrayCount, 0) $=$ TP_VPAS

Yipos $=272+$ Abs(Blk_WorkSpace.Top)

End Select

Get_Function_Pic Pic_Work, FuncCode(ArrayCount, 0)

Initīil_PicContrl Pic_Work, Xipos, Yipos

End Sub

Sub Tool_Blk5_Click ()

ArrayCount $=$ ArrayCount +1

Xipos $=152+$ Abs(Blk_WorkSpace.Left)

Select Case CurrentTool

Case TP_COM1 'DSP

FuncCode(ArrayCount, 0) $=$ TP_BWND4

Yipos $=170+$ Abs(Blk_WorkSpace.Top)

Case TP_COM3 'VAI

FuncCode(ArrayCount, 0) $=$ TP_VMXM

Yipos $=238+$ Abs(Blk_WorkSpace.Top)

End Select

Get_Function_Pic Pic_Work, FuncCode(ArrayCount, 0)

Initial_PicContrl Pic_Work, Xipos, Yipos

End Sub

Sub Tool_Blk6_Click ()

ArrayCount $=$ ArrayCount +1

Xipos $=190+$ Abs(Blk_WorkSpace.Left)

Yipos $=170+$ Abs(Blk_WorkSpace.Top)

FuncCode(ArrayCount, 0) = TP_BCFIR

Get_Function_Pic Pic_Work, FuncCode(ArrayCount, 0)

Initial_PicContrl Pic_Work, Xipos, Yipos

End Sub

Sub Tool_Blk7_Click ()

ArrayCount $=$ ArrayCount +1

Xipos $=228+$ Abs(Blk_WorkSpace.Left)

Yipos $=170+$ Abs(Blk_WorkSpace.Top)

FuncCode(ArrayCount, 0 ) = TP_BDFIR

Get_Function_Pic Pic_Work, FuncCode(ArrayCount, 0)

Initial_PicContrl Pic_Work, Xipos, Yipos

End Sub 
Sub Tool_Blk8_Click ()

ArrayCount $=$ ArrayCount +1

Xipos $=266+$ Abs(Blk_WorkSpace.Left)

Yipos $=170+$ Abs(Blk_WorkSpace.Top)

FuncCode(ArrayCount, 0 ) $=$ TP_BRFIR

Get_Function_Pic Pic_Work, FuncCode(ArrayCount, 0)

Initial_PicContrl Pic_Work, Xipos, Yipos

End Sub

Sub Tool_Blk9_Click ()

ArrayCount $=$ ArrayCount +1

Xipos $=304+$ Abs(Blk_WorkSpace.Left)

Yipos $=170+$ Abs(Blk_WorkSpace.Top)

FuncCode(ArrayCount, 0 ) $=$ TP_BFCT

Get_Function_Pic Pic_Work, FuncCode(ArrayCount, 0)

Initial_PicContrl Pic_Work, Xipos, Yipos

End Sub

Sub Tool_Com1_Click (Value As Integer)

If Value Then

MainEditor.MousePointer $=$ DEFAULT

CurrentTool $=\mathrm{TP}$ COM 1

ToollnProcess $=$ True

Select Case TP TID

Case TP_SY $\overline{\mathrm{S}}$

PFlag $=$ PFlag -1

If PFlag $<0$ Then

Exit Sub

Else

Pan_NSys.Caption $=$ Format $\$($ PFlag $)$

End If

Count)

If Pic_SysWork.Visible Then Prepare_New_Sys_Icon XSI(SICount), YSI(SI-

Display_Sys_Icon CurrentTool

Case TP_BLK

'Wrong instruction or no extension icon selected. Turn extension off

If ToolPalette_BlkExt.Visible Then ToolPalette BlkExt.Visible $=$ False

If Pic_Work.Visible Then Prepare_New_Icon XArr(ArraySiz), YArr(ArraySiz)

ToolPalette_BlkExt.Move Abs(Blk_WorkSpace.Left), $170+$

Abs(Blk_WorkSpace.Top), 419, 52

toolpalette icons

ToolPalette Block Initialize 'Redraw the first few blocks of

ToolPalette_BlkExt. Visible $=$ True

End Select

End If

End Sub

Sub Tool_Com2_Click (Value As Integer) 


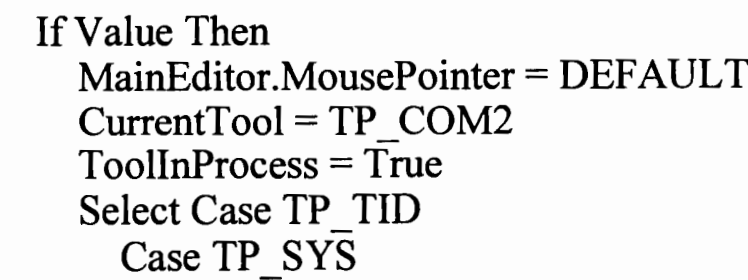

Count)

If Pic_SysWork.Visible Then Prepare_New_Sys_Icon XSI(SICount), YSI(SI-

Display_Sys_Icon CurrentTool

Case TP_BLK

'Wrong instruction or no extension icon selected. Turn extension off

If ToolPalette_BlkExt.Visible Then ToolPalette_BlkExt.Visible $=$ False

If Pic Work.Visible Then Prepare New_Icon XArr(ArraySiz), YArr(ArraySiz)

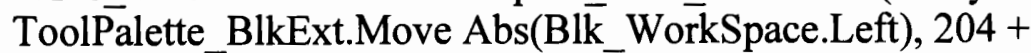

Abs(Blk_WorkSpace.Top), 153, 52

ToolPalette_Block_Initialize

End Select

ToolPalette_BlkExt. Visible $=$ True

End If

End Sub

Sub Tool_Com3_Click (Value As Integer)

If Value Then

MainEditor.MousePointer $=$ DEFAULT

CurrentTool $=\mathrm{TP}$ COM3

ToollnProcess $=$ True

Select Case TP TID

Case TP_SYS

Count)

If Pic_SysWork.Visible Then Prepare_New_Sys_Icon XSI(SICount), YSI(SI-

Display_Sys_Icon Current Tool

Case TP BLK

If ToolPalette BlkExt.Visible Then ToolPalette BlkExt.Visible $=$ False

If Pic_Work.Visible Then Prepare_New_Icon XArr(ArraySiz), YArr(ArraySiz)

ToolPalette_BlkExt.Move Abs(Blk_WorkSpace.Left), $238+$

Abs(Blk_WorkSpace.Top), 191, 52

ToolPalette_Block_Initialize

ToolPalette_BlkExt.Visible $=$ True

End Select

End If

End Sub

Sub Tool_Com4_Click (Value As Integer)

If Value Then

MainEditor.MousePointer $=$ DEFAULT

CurrentTool $=$ TP_COM4

ToolinProcess $=$ True

Select Case TP_TID 


\section{Case TP_SYS}

Count)

If Pic_SysWork.Visible Then Prepare_New_Sys_Icon XSI(SICount), YSI(SI-

\section{Display_Sys_Icon CurrentTool}

Case TP BLK

If ToolPalette BlkExt.Visible Then ToolPalette BlkExt.Visible $=$ False

If Pic_Work. Visible Then Prepare_New_Icon XArr(ArraySiz), YArr(ArraySiz)

ToolPalette_BlkExt.Move Abs(Blik_WorkSpace.Left), $272+$

Abs(Blk_WorkSpace.Top), 153, 52

ToolPalette_Block_Initialize

End Select

ToolPalette_BlkExt.Visible $=$ True

End If

End Sub

Sub Tool_Com5_Click (Value As Integer)

If Value Then

MainEditor.MousePointer $=$ DEFAULT

CurrentTool $=$ TP COM5

ToolInProcess $=$ True

Select Case TP_TID

Case TP_SYS

'No icon is to be displayed

Case TP_BLK

If ToolPalette_BlkExt.Visible Then ToolPalette_BlkExt.Visible $=$ False

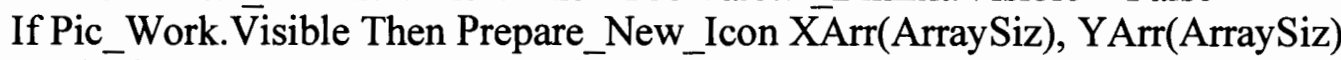

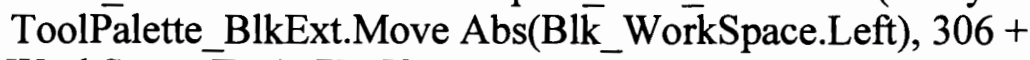

Abs(Blk_WorkSpace.Top), 77, 52

ToolPalette_Block_Initialize

End Select

ToolPalette_BlkExt.Visible $=$ True

End If

End Sub

Sub Tool_Com6_Click (Value As Integer)

If Value Then

MainEditor.MousePointer $=$ DEFAULT

Current Tool $=$ TP_COM6

ToolInProcess $=$ True

Select Case TP_TID

Case TP_SY $\bar{S}$

Not icon is to be displayed

Case TP_BLK

If ToolPalette_BlkExt.Visible Then ToolPalette BlkExt.Visible $=$ False

If Pic_Work. Visible Then Prepare_New_Icon XArr(ArraySiz), YArr(ArraySiz)

ToolPalette_BlkExt.Move Abs(Blik_WorkSpace.Left), $340+$

Abs(Blk_WorkSpace.Top), 77, 52

ToolPalette_Block_Initialize 
End Select

ToolPalette_BlkExt.Visible $=$ True

Else

End If

End Sub

Sub Tool_Line_Click (Value As Integer)

If IniDraw And (CurrentTool $=$ TP_LINE Or CurrentTool $=$ TP_UNLINE) Then

If CurrentTool $=$ TP LINE Then

If TP_TID $=$ TP_BLK Then

DrawLine Blk_WorkSpace, StartX, StartY, OldX, OldY

$\operatorname{Hpts}($ LineCount, 1$)=0$

Vpts(LineCount, 1$)=0$

ElseIf TP TID $=$ TP_SYS Then

DrawLine Sys_WorkSpace, StartX, StartY, OldX, OldY

$\mathrm{XPts}($ LCount, $\overline{1})=0$

YPts(LCount, 1$)=0$

End If

End If

IniDraw $=$ False: FinDraw $=$ False

DisLineX1 $=0$ : DisLineY1 $=0$

StartEvent $=$ False

End If

If Value Then

MainEditor.MousePointer $=$ DEFAULT

CurrentTool $=$ TP LINE

Select Case TP TID

Case TP BLK

'If the Block ToolPalette Extension is visible, hide it

If ToolPalette BlkExt.Visible Then ToolPalette BlkExt.Visible $=$ False

'Prepare for draw line. Turn off the Pic Work Container

If Pic_Work.Visible Then Prepare_New_Icon XArr(ArraySiz), YArr(ArraySiz)

'turn pic_work icon OFF

Case TP_SYS

'Prepare for draw line. Turn off the Pic_SysWork Container

If Pic_SysWork.Visible Then Prepare_New_Sys_Icon XSI(SICount), YSI(SI-

Count) 'turn pic_work icon OFF

End Select

ScreenInfo(ScrnStage) . Changed $=$ True

ToolInProcess $=$ True

End If

End Sub

Sub Tool_Reset_Click (Value As Integer)

'Wrong instruction or no extension icon selected. Turn extension off

If ToolPalette_BlkExt.Visible Then ToolPalette_BlkExt.Visible $=$ False

If IniDraw And (CurrentTool $=$ TP_LINE Or CurrentTool $=$ TP_UNLINE) Then

If CurrentTool $=$ TP_LINE Then 


$$
\text { If TP_TID = TP_BLK Then }
$$

DrawLine Blk_WorkSpace, StartX, StartY, OldX, OldY

Hpts(LineCount, 1) $=0$

$\operatorname{Vpts}($ LineCount, 1$)=0$

ElseIf TP_TID $=$ TP_SYS Then

DrawLine Sys_WorkSpace, StartX, StartY, OldX, OldY

$\mathrm{XPts}($ LCount, $\overline{1})=0$

End If

YPts (LCount, 1) $=0$

End If

IniDraw $=$ False: FinDraw $=$ False

DisLineX1 $=0$ : DisLineY1 $=0$

StartEvent $=$ False

End If

If Value Then

MainEditor.MousePointer $=$ DEFAULT

CurrentTool $=$ TP_RESET

Msg = "Are you sure that all icons \& lines are to be erased?"

If MsgBox(Msg, 49, "ERASE ALL") = 1 Then

End If

Initialize_All_Variables TP_TID

Tool_Select.Value $=$ True

End If

End Sub

'The Select button is always depress when the other tool button is not depress.

'The select button allows the icon to drag and edit the Pic_Work.

Sub Tool_Select_Click (Value As Integer)

If IniDraw And (CurrentTool $=$ TP_LINE Or CurrentTool $=$ TP_UNLINE) Then

If CurrentTool $=$ TP LINE Then

If TP_TID $=$ TP $\bar{B} L K$ Then

DrawLine Blk_WorkSpace, StartX, StartY, OldX, OldY

Hpts(LineCount, 1) $=0$

$\mathrm{Vpts}($ LineCount, 1$)=0$

ElseIf TP TID $=$ TP SYS Then

DrawLine Sys_WorkSpace, StartX, StartY, OldX, OldY

$\mathrm{XPts}($ LCount, $\overline{1})=0$

YPts $($ LCount, 1$)=0$

End If

End If

IniDraw $=$ False: FinDraw $=$ False

DisLineX1 $=0$ : DisLineY1 $=0$

StartEvent $=$ False

End If

If Value Then

MainEditor.MousePointer $=$ DEFAULT 
Select Case TP TID

Case TP_SY $\bar{S}$

If CurrentTool $=$ TP_TEXT Then

TxtUnFocus STxtSelected

If STxtEdit(STxtSelected).Text = "" Then

If STxtSelected $=$ STxtNum -1 Then

STxtNum $=$ STxtNum -1

If STxtNum $<0$ Then STxtNum $=0$

If STxtNum $-1<0$ Then STxtSelected $=0$ Else STxtSelected $=$ STxtNum -1

Num). Visible $=$ False

If STxtNum $>1$ Then Unload STxtEdit(STxtNum) Else STxtEdit(STxt-

STxtEnable $=$ False

Else

If Not STxtEnable Then STxtEdit(STxtSelected).Visible $=$ False

End If

End If

Sys_WorkSpace.SetFocus

End If

If Not Pic_SysWork.Visible And SISiz $>0$ Then

Pic_SysWork.Move XSI(SICount), YSI(SICount)

Get_Function_Pic Pic_SysWork, FuncCode(SICount, 1)

Pic_SysWork.Visible $=$ True

NESS)

$\mathrm{R}=$ PatBlt(Sys_WorkSpace.hDC, XSI(SICount), YSI(SICount), 34, 54, WHITE-

\section{End If}

Case TP_BLK

If CurrentTool $=$ TP_TEXT Then

TxtUnFocus TxtSelected

If TxtEdit(TxtSelected).Text = "" Then

If TxtSelected $=$ TxtNum -1 Then

If TxtSelected $>1$ Then Unload TxtEdit(TxtSelected) Else TxtEdit(Txt-

Selected). Visible $=$ False

TxtEnable $=$ False

TxtNum $=$ TxtNum -1

If TxtNum $<=0$ Then

TxtNum $=0$

TxtSelected $=0$

Else

TxtSelected $=$ TxtNum -1

End If

Else

If Not TxtEnable Then TxtEdit(TxtSelected).Visible $=$ False End If

End If

'Blk_WorkSpace.SetFocus

End If

'If the Block ToolPalette Extension is visible, hide it

If ToolPalette_BlkExt.Visible Then ToolPalette_BlkExt.Visible $=$ False 
'Move the picture container to the new icon location.

If Not Pic_Work.Visible And ArrayCount $>0$ Then

Pic Work.Move XArr(ArraySiz), YArr(ArraySiz)

Get Function_Pic Pic_Work, FuncCode(ArraySiz, 0)

Pic_Work.Visible $=$ True WHITENESS)

$\mathrm{R}=$ PatBlt(Blk_WorkSpace.hDC, XArr(ArraySiz), YArr(ArraySiz), 34, 54,

End I $\overline{\mathbf{f}}$

End Select

CurrentTool $=$ TP SELECT

ToolInProcess $=\overline{\text { False }}$

End If

End Sub

Sub Tool_Text_Click (Value As Integer)

If IniDraw And (CurrentTool $=$ TP_LINE Or CurrentTool $=$ TP_UNLINE) Then If CurrentTool $=$ TP LINE Then

If TP_TID $=$ TP_BLK Then

DrawLine Blk_WorkSpace, StartX, StartY, OldX, OldY

Hpts(LineCount, 1) $=0$

Vpts(LineCount, 1) $=0$

ElseIf TP TID $=$ TP_SYS Then

DrawLine Sys_WorkSpace, StartX, StartY, OldX, OldY

$\mathrm{XPts}($ LCount, $\overline{1})=0$

YPts(LCount, 1) $=0$

End If

End If

IniDraw $=$ False: FinDraw $=$ False

DisLineX1 $=0$ : DisLineY1 $=0$

StartEvent $=$ False

End If

If Value Then

Select Case TP_TID

Case TP_BLK

'If the Block ToolPalette Extension is visible, hide it

If ToolPalette_BlkExt.Visible Then ToolPalette_BlkExt.Visible $=$ False

'Prepare for erase of line. Turn off the Pic_Work Container

If Pic_Work.Visible Then Prepare_New_Icon XArr(ArraySiz), YArr(ArraySiz)

Case TP_SYS

'Prepare for erase of line. Turn off the Pic_Work Container

Count)

If Pic_SysWork.Visible Then Prepare_New__Sys_Icon XSI(SICount), YSI(SI-

End Select

CurrentTool $=$ TP_TEXT

MainEditor.MousePointer = I_BEAM

End If

End Sub 
Sub Tool_UnLine_Click (Value As Integer)

If IniDraw And (CurrentTool $=$ TP_LINE Or CurrentTool $=$ TP_UNLINE) Then If CurrentTool $=$ TP LINE Then

If TP_TID $=$ TP_BLK Then

DrawLine Blk_WorkSpace, StartX, StartY, OldX, OldY

Hpts(LineCount, 1) $=0$

Vpts(LineCount, 1) $=0$

ElseIf TP TID = TP SYS Then

DrawLine Sys_WorkSpace, StartX, StartY, OldX, OldY

$\mathrm{XPts}($ LCount, 1$)=0$

YPts(LCount, 1) $=0$

End If

End If

IniDraw $=$ False: FinDraw $=$ False

DisLineX1 $=0$ : DisLineY1 $=0$

StartEvent $=$ False

End If

If Value Then

CurrentTool $=$ TP_UNLINE

Select Case TP_TID

Case TP_BLK

'If the Block ToolPalette Extension is visible, hide it

If ToolPalette_BlkExt.Visible Then ToolPalette_BlkExt.Visible $=$ False

'Prepare for erase of line. Turn off the Pic_Work Container

If Pic_Work.Visible Then Prepare_New_Icon XArr(ArraySiz), YArr(ArraySiz)

Case TP_SYS

'Prepare for erase of line. Turn off the Pic_Work Container

Count)

If Pic_SysWork.Visible Then Prepare_New_Sys_Icon XSI(SICount), YSI(SI-

End Select

ScreenInfo(ScrnStage) . Changed $=$ True

ToolInProcess $=$ True

End If

End Sub

Sub ToolPalette_BlkExt_Paint ()

Tool_Blk1.Move 0, 0, 38, 38

Tool_Blk2.Move 38, 0, 38, 38

Tool_Blk3.Move 76, 0, 38, 38

Tool_Blk4.Move 114, 0, 38, 38

Tool_Blk5.Move 152, 0, 38, 38

Tool_Blk6.Move 190, 0, 38, 38

Tool_Blk7.Move 228, 0, 38, 38

Tool_Blk8.Move 266, 0, 38, 38

Tool Blk9.Move 304, 0, 38, 38

Tool_Blk10.Move 342, 0, 38, 38

Tool_Blk11.Move 380, 0, 38, 38 
ToolPalette_BlkExt.CurrentX $=0$

ToolPalette_BlkExt.CurrentY $=$ ToolPalette_BlkExt.ScaleTop +38

Select Case CurrentTool

Case TP COM1 'DSP

ToolPalette_BlkExt.Print " BFLY2"; " BFLY4"; " BFLY16";

ToolPalette_BlkExt.Print " BWND2"; " BWND4"; " BCFIR";

ToolPalette_BlkExt.Print " BDFIR"; " BRFIR"; " BFCT";

ToolPalette BlkExt.Print " BFCT2"; " BRFT"

Case TP COM 2 'CAI

ToolPalette_BlkExt.Print " CADD"; " CSUB"; " CMUL"; " CMAG"

Case TP_COM 3 'VAI

ToolPälette_BlkExt.Print " VADD"; " VSUB"; " VMUL";

ToolPalette_BlkExt.Print " VABS"; " VMXM"

Case TP_COM 4 'VLI

ToolPalette_BlkExt.Print "VNAND"; " VNOR"; " VXNOR";

ToolPalette-BlkExt.Print " VPAS"

Case TP_COM 5 'GPI

ToolPalette BlkExt.Print " MOVD"; " MOVC"

Case TP_COM̄ 6 'MISC

End Select

ToolPalette_BlkExt.Print " Signal"; " Display"

End Sub

'This routine is to select the icon picture from the PicClip and assign

'it to each individual tool_com icons when user click on it.

Sub ToolPalette_Block_Initialize ()

MainEditor.PicClip $\overline{\text { I }}$ cons.ClipWidth $=32$

Select Case CurrentTool

Case TP_COM1 'DSP

PicClip_Icons.ClipY $=0$

PicClip_Icons.ClipX $=0$

Tool_Blk1.Picture $=$ PicClip_Icons.Clip

PicClip_Icons.ClipX $=32$

Tool Blk2.Picture $=$ PicClip_Icons.Clip

PicClip_Icons.ClipX $=64$

Tool_Blk3.Picture $=$ PicClip_Icons.Clip

PicClip_Icons.ClipX $=96$

Tool_Bīk4.Picture $=$ PicClip_Icons.Clip

PicClip_Icons.ClipX $=128$

Tool_Blk5.Picture $=$ PicClip_Icons.Clip

Case T $\overline{\mathrm{P}}$ COM2 'CAI

PicClip_Icons.ClipY $=32$

PicClip_Icons.ClipX $=96$

Tool_Blk1 1 Picture $=$ PicClip_Icons.Clip

PicClip_Icons.ClipX $=128$

Tool_Blk2.Picture $=$ PicClip_Icons.Clip

PicClip_Icons.ClipX $=160$ 
Tool_Blk3.Picture $=$ PicClip_Icons.Clip

PicClip_Icons.ClipX $=192$

Tool_Blk4.Picture $=$ PicClip_Icons.Clip Case T $\overline{\mathrm{P}}$ COM 3 'VAI

PicClip_Icons.ClipY $=32$

PicClip_Icons.ClipX $=224$

Tool Blk 1 .Picture $=$ PicClip_Icons.Clip

PicClip_Icons.ClipY $=64$

PicClip_Icons.ClipX $=0$

Tool_Blk2.Picture $=$ PicClip_Icons.Clip

PicClip_Icons.ClipX $=32$

Tool_Blk3.Picture $=$ PicClip_Icons.Clip

PicClip_Icons.ClipX $=64$

Tool_Blk4.Picture $=$ PicClip_Icons.Clip

PicClip_Icons.ClipX $=96$

Tool_Blk5.Picture $=$ PicClip_Icons.Clip

Case TP COM4 'VLI

PicClip_Icons.ClipY $=64$

PicClip_Icons.ClipX $=128$

Tool_Blk1.Picture $=$ PicClip_Icons.Clip

PicClip_Icons.ClipX $=160$

Tool_Bl̄k2.Picture $=$ PicClip_Icons.Clip

PicClip_Icons.ClipX $=192$

Tool_Bīk3.Picture $=$ PicClip_Icons.Clip

PicClip Icons.ClipX $=224$

Tool Blk4.Picture $=$ PicClip_Icons.Clip

Case Tर COM5 'GPI

PicClip_Icons.ClipY $=96$

PicClip_Icons.ClipX $=0$

Tool_Blk1.Picture $=$ PicClip_Icons.Clip

PicClip_Icons.ClipX $=32$

Tool Blk2.Picture $=$ PicClip_Icons.Clip

Case TP COM6 'MISC

PicClip_Icons.ClipY $=128$

PicClip_Icons.ClipX $=192$

Tool_Blk1.Picture $=$ PicClip_Icons.Clip

PicClip_Icons.ClipY $=160$

PicClip_Icons.ClipX $=0$

End Select

Tool_Bl̄k2.Picture $=$ PicClip_Icons.Clip

End Sub

Sub ToolPalette_Icons_Initialize ()

'ToolPalette Icons Selection. Visible $=$ True

PicClip_Icons.ClipHeight $=32$

PicClip_Icons.ClipWidth $=32$

Tool_Select.PictureUp $=$ LoadPicture ()

Tool_Line.PictureUp $=$ LoadPicture() 
Tool_Com1.PictureUp $=$ LoadPicture()

Tool_Com2.PictureUp $=$ LoadPicture ()

Tool_Com3.PictureUp $=$ LoadPicture ()

Tool_Com4.PictureUp $=$ LoadPicture()

Tool Com5.PictureUp $=$ LoadPicture()

Tool_Com6.PictureUp = LoadPicture()

Select Case TP TID

Case TP MĀIN

ToolPălette Icons_Selection. Visible $=$ False

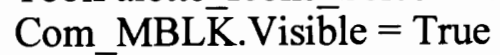

Com_MSYS.Visible $=$ True

Start System. Visible $=$ False

Stop_System.Visible $=$ False

Sys_System. Visible $=$ False

Case TP BLK

Com_MBLK.Visible $=$ False

Com_MSYS.Visible $=$ False

Start System. Visible $=$ True

Stop_System.Visible $=$ True

Sys System. Visible $=$ True

ToolPalette_Icons_Selection. Visible $=$ True

'Tool_Palette_OnOff TP_BLK

PicClip_Icons.ClipY $=\overline{96}$

'Insert Select icon

PicClip_Icons.ClipX $=64$

Tool_Select.PictureUp $=$ PicClip_Icons.Clip

'Insert Line icon

PicClip_Icons.ClipX $=96$

Tool_Line.PictureUp $=$ PicClip_Icons.Clip

PicClip_Icons.ClipY $=160$

PicClip_Icons.ClipX $=32$

Tool_Com1.PictureUp $=$ PicClip_Icons.Clip

PicClip_Icons.ClipX $=64$

Tool_Com2.PictureUp $=$ PicClip_Icons.Clip

PicClip_Icons.ClipX $=96$

Tool_Com3.PictureUp $=$ PicClip_Icons.Clip

PicClip_Icons.ClipX $=128$

Tool_Com4.PictureUp = PicClip_Icons.Clip

If Not Tool_Com5.Visible Then Tool_Com5.Visible $=$ True

If Not Tool_Com6.Visible Then Tool_Com6.Visible $=$ True

PicClip_Icons.ClipX $=160$

Tool_Com5.PictureUp $=$ PicClip_Icons.Clip

PicClip_Icons.ClipX $=192$

Tool_Com6.PictureUp $=$ PicClip_Icons.Clip

Case TP $\mathrm{P}$ SYS

Com_MSYS.Visible $=$ False

Com_MBLK.Visible $=$ False

Start_System.Visible $=$ True 
Stop_System.Visible $=$ True

Sys_System.Visible $=$ True

ToolPalette_Icons_Selection.Visible $=$ True

'Tool_Palette_OnOff TP_SYS

PicClip_Icons. ClipY $=\overline{96}$

'Insert Select icon

PicClip_Icons.ClipX $=64$

Tool_Select.PictureUp $=$ PicClip_Icons.Clip

'Insert Line icon

PicClip_Icons.ClipX $=96$

Tool_Line.PictureUp $=$ PicClip_Icons.Clip

'Insert the LH9124 icon

PicClip_Icons.ClipY $=128$

PicClip_Icons.ClipX $=160$

Tool_Com1.PictureUp = PicClip_Icons.Clip

'Insert the LH9320 \& memory icon

PicClip_Icons.ClipY $=192$

PicClip_Icons.ClipX $=64$

Tool_Com2.PictureUp $=$ PicClip_Icons.Clip

'Insert the Signal icon

PicClip_Icons.ClipY $=128$

PicClip_Icons.ClipX $=192$

Tool_Com3.PictureUp $=$ PicClip_Icons.Clip

'Insert the Display icon

PicClip_Icons.ClipY $=160$

PicClip_Icons.ClipX $=192$

Tool_Com4.PictureUp = PicClip_Icons.Clip

'Turn off the last two icons

If Tool_Com5.Visible Then Tool_Com5.Visible $=$ False

End Select

If Tool_Com6.Visible Then Tool_Com6.Visible $=$ False

End Sub

Sub TxtEdit_Change (Index As Integer)

TxtUnFocus Index

TxtEdit(Index $) \cdot$ Height $=$ TextHeight $($ TxtEdit(Index $))+4$

TxtEdit(Index).Width $=$ TextWidth(TxtEdit(Index) $) * 1.4+20$

If TxtEdit(Index).Text $\diamond$ "' Then TxtFocus Index

TxtEnable $=$ False

End Sub

Sub TxtEdit_GotFocus (Index As Integer)

TxtUnFocus TxtSelected

If TxtEdit(TxtSelected). Text $=$ "" Then

If Not TxtEnable Then TxtEdit(TxtSelected). Visible $=$ False

End If

Blk WorkSpace.Print Err, TxtFocus Index 
TxtSelected $=$ Index

End Sub

Sub TxtEdit_MouseDown (Index As Integer, Button As Integer, Shift As Integer, X As Single, Y As Single)

If Button $=$ LEFT BUTTON And CurrentTool $=$ TP_SELECT Then

TxtEdit(Index).Drag 1

DragX $=\mathrm{X} / 15$

DragY $=\mathrm{Y} / 15$

End If

Blk_WorkSpace.SetFocus

End Sub

Sub TxtEdit_MouseMove (Index As Integer, Button As Integer, Shift As Integer, X As Single, Y As Single)

TxtEdit(Index) .MousePointer $=$ IIf $($ CurrentTool $=$ TP_SELECT, ARROW, DEFAULT $)$ End Sub

Sub TxtEdit_MouseUp (Index As Integer, Button As Integer, Shift As Integer, X As Single, Y As Single)

If Button $=$ LEFT_BUTTON And CurrentTool $=$ TP_SELECT Then TxtEdit(Index). Drag 2

End Sub

Sub Write_Script (XID As Integer)

On Error Resume Next

Dim LongName As String

Dim Title As String

Dim CDPath As String

If TP_TID = TP_BLK And SavFlag Then

XInit = XArr(ArraySiz): YInit = YArr(ArraySiz)

If Pic_Work.Visible Then Prepare_New_Icon XInit, YInit

ElseIf TP_TID $=$ TP_SYS And SavFlag Then

XInit $=$ XSI(SICount): YInit $=$ YSI (SICount $)$

If Pic_SysWork.Visible Then Prepare_New_Sys_Icon XInit, YInit

End If

If XID = MID_WRITE_SCRIPT Then

' Set default filter.

MainEditor.CMDialog_Edit.Filter $=$ "System Schedule Files $\left.\left({ }^{*}\right.$.ssf $)\right|^{*}$.SSF"

' Display Save As dialog.

MainEditor.CMDialog_Edit.FilterIndex $=1$

MainEditor.CMDialog_Edit.DefaultExt = "SSF"

If Right(Script_Path, 1) $\diamond " \mid "$ Then

MainEditor. $\bar{C} M D$ ialog_Edit.Filename $=$ Script_Path \& "।" \& Script_FName End If

' If file has not been saved yet, set common dialog's

' default file name to *.ssf

If Script_FName $=$ "" Then 
MainEditor.CMDialog_Edit.Filename $=" *$.ssf"

End If

MainEditor.CMDialog_Edit.Action $=2$

' Exit if user cancels the dialog.

If Err $=32755$ Then Exit Sub

Title $=$ MainEditor.CMDialog_Edit.Filetitle

LongName $=$ MainEditor.CMDialog_Edit.Filename

Script_Path $=$ Left(LongName, Len(LongName) - Len(Title))

Script_FName $=$ MainEditor.CMDialog_Edit.Filetitle

' Adjust for root directory.

If Len(Script Path) $=3$ Then

CDPath $=$ Script_Path

Else

CDPath $=$ Left(LongName, Len(LongName) - Len(Title) -1$)$

End If

' Change directories for the editor and the viewer.

ChDir CDPath

End If

MainEditor.MousePointer $=$ HOURGLASS

Extract_AG_File Script_Path, Script_FName, I

For $I=1$ To ArrayCount

System_Scheduler_File Script_Path, Script_FName, I Next I

MainEditor.MousePointer $=$ DEFAULT

End Sub 